Prepared in cooperation with the Arkansas Natural Resources Commission and the Arkansas Geological Survey

\title{
Water Levels and Selected Water-Quality Conditions in the Mississippi River Valley Alluvial Aquifer in Eastern Arkansas, 2008
}

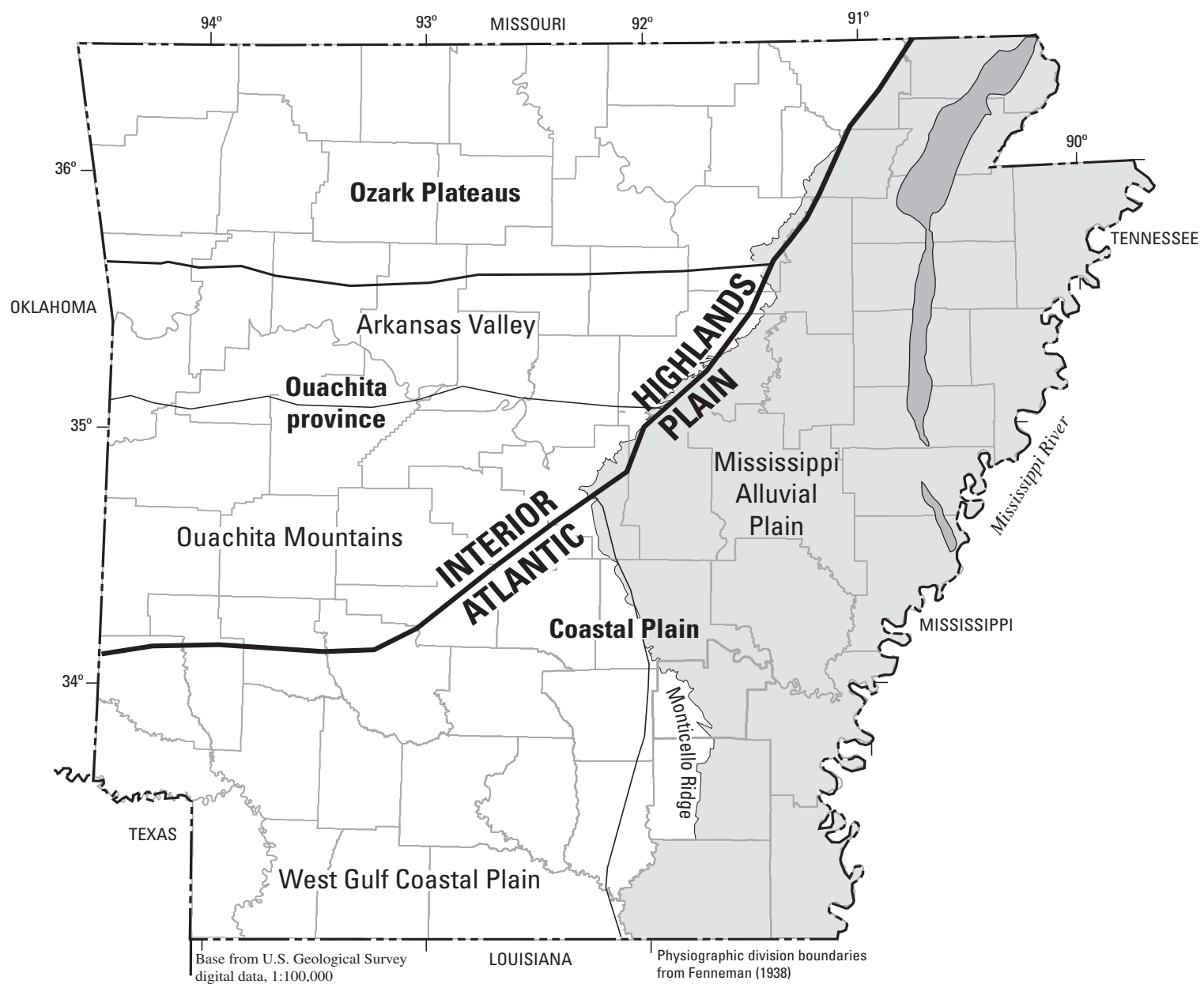

Scientific Investigations Report 2010-5140 


\section{Water Levels and Selected Water-Quality Conditions in the Mississippi River Valley Alluvial Aquifer in Eastern Arkansas, 2008}

By T.P. Schrader

Prepared in cooperation with the Arkansas Natural Resources Commission and the Arkansas Geological Survey

Scientific Investigations Report 2010-5140 


\section{U.S. Department of the Interior \\ KEN SALAZAR, Secretary \\ U.S. Geological Survey \\ Marcia K. McNutt, Director}

U.S. Geological Survey, Reston, Virginia: 2010

This and other USGS information products are available at http://store.usgs.gov/
U.S. Geological Survey
Box 25286 , Denver Federal Center
Denver, CO 80225
To learn about the USGS and its information products visit http://www.usgs.gov/
1-888-ASK-USGS

Any use of trade, product, or firm names is for descriptive purposes only and does not imply endorsement by the U.S. Government.

Although this report is in the public domain, permission must be secured from the individual copyright owners to reproduce any copyrighted materials contained within this report.

Suggested citation:

Schrader, T.P., 2010, Water levels and selected water-quality conditions in the Mississippi River Valley alluvial aquifer in eastern Arkansas, 2008: U.S. Geological Survey Scientific Investigations Report 2010-5140, 71 p., 2 plates. 


\section{Contents}

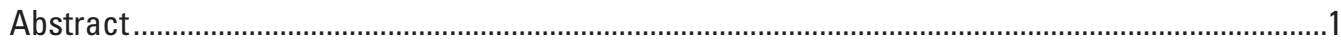

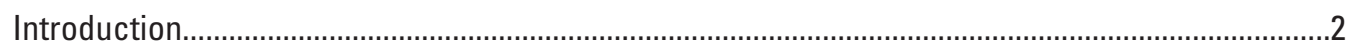

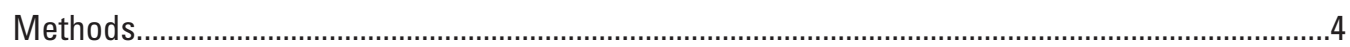

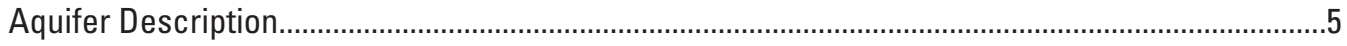

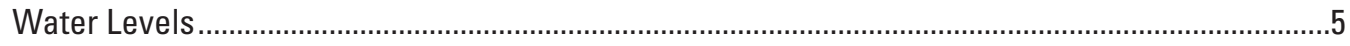

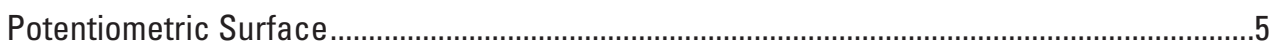

Water-Level Difference from 2004 to 2008

Long-Term Water-Level Changes...................................................................................

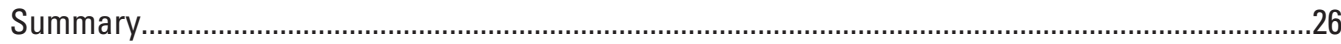

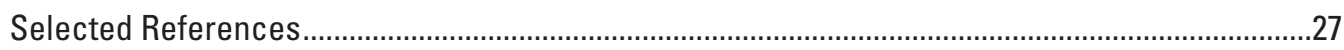

Appendix 1. Information pertaining to water levels measured in wells completed in the Mississippi River Valley alluvial aquifer in eastern Arkansas, spring 2008........................30

Appendix 2. Information pertaining to water levels measured in 2004 and 2008 in the Mississippi River Valley alluvial aquifer in eastern Arkansas ............................................50

Appendix 3. Specific conductance and temperature data from wells completed in the Mississippi River Valley alluvial aquifer in eastern Arkansas, summer 2008.

\section{Plates}

[In pocket]

1. Map showing potentiometric surface of the alluvial aquifer, spring 2008

2. Map showing difference in water level in the alluvial aquifer

\section{Figures}

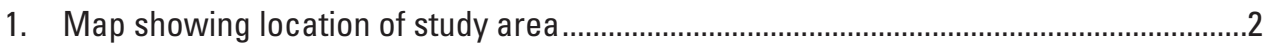

2. Graph showing estimated withdrawals from the Mississippi River Valley alluvial

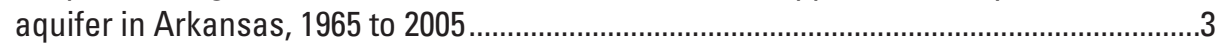

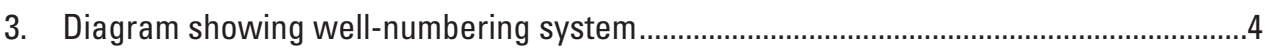

4. Water-level hydrographs (A to CC) for selected wells in the Mississippi River Valley alluvial aquifer ......................................................................................................

5. Graph showing distribution of specific conductance in samples from the Mississippi

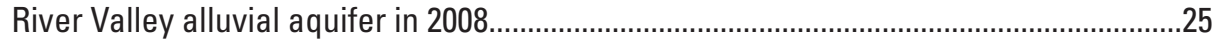

6. Graph showing distribution of specific conductance in samples from the Mississippi River Valley alluvial aquifer in 2006...........................................................................25

\section{Tables}

1. Range, mean, median, and correlation coefficient of annual rise-decline in water level by county for wells in the Mississippi River Valley alluvial aquifer, 1984-2008 ........8 


\section{Conversion Factors Vertical Datums, and Abbreviations}

\begin{tabular}{lll}
\hline \multicolumn{1}{c}{ Multiply } & \multicolumn{1}{c}{ By } & \multicolumn{1}{c}{ To obtain } \\
\hline inch (in.) & \multicolumn{2}{c}{ Length } \\
foot (ft) & 2.54 & centimeter $(\mathrm{cm})$ \\
mile (mi) & 0.3048 & meter $(\mathrm{m})$ \\
\hline \multicolumn{3}{c}{ Flow rate } \\
\hline foot per year (ft/yr) & 1.609 & kilometer $(\mathrm{km})$ \\
cubit foot per second (ft $3 / \mathrm{s})$ & 0.3048 & meter per year $(\mathrm{m} / \mathrm{yr})$ \\
million gallons per day (Mgal $/ \mathrm{d})$ & 3.02832 & cubic meter per second $\left(\mathrm{m}^{3} / \mathrm{s}\right)$ \\
foot per year (ft/yr) & 0.3048 & liter per day $(\mathrm{L} / \mathrm{d})$ \\
\hline
\end{tabular}

Temperature in degrees Celsius $\left({ }^{\circ} \mathrm{C}\right)$ may be converted to degrees Fahrenheit $\left({ }^{\circ} \mathrm{F}\right)$ as follows:

${ }^{\circ} \mathrm{F}=\left(1.8 \mathrm{x}^{\circ} \mathrm{C}\right)+32$

Temperature in degrees Fahrenheit $\left({ }^{\circ} \mathrm{F}\right)$ may be converted to degrees Celsius $\left({ }^{\circ} \mathrm{C}\right)$ as follows:

${ }^{\circ} \mathrm{C}=\left({ }^{\circ} \mathrm{F}-32\right) / 1.8$

Vertical coordinate information is referenced to the National Geodetic Vertical Datum of 1929 (NGVD 1929).

Horizontal coordinate information is referenced to the North American Datum of 1983 (NAD 1983).

Altitude as used in this report, refers to distance above the vertical datum.

Specific conductance is given in microsiemens per centimeter at 25 degrees Celsius $\left(\mu \mathrm{S} / \mathrm{cm}\right.$ at $25^{\circ} \mathrm{C}$ ). 


\title{
Water Levels and Selected Water-Quality Conditions in the Mississippi River Valley Alluvial Aquifer in Eastern Arkansas, 2008
}

\author{
By T.P. Schrader
}

\section{Abstract}

During the spring of 2008, the U.S. Geological Survey, in cooperation with the Arkansas Natural Resources Commission and the Arkansas Geological Survey, measured 670 water levels in 659 wells completed in the Mississippi River Valley alluvial aquifer in eastern Arkansas. Groundwater levels are affected by groundwater withdrawals resulting in potentiometric-surface depressions. In 2008, the lowest water-level altitude was 69 feet above National Geodetic Vertical Datum of 1929 in the center of Arkansas County. The highest waterlevel altitude was 288 feet above National Geodetic Vertical Datum of 1929 in northeastern Clay County on the west side of Crowleys Ridge. Two large depressions in the potentiometric surface are located in Arkansas, Lonoke, and Prairie Counties and west of Crowleys Ridge in Craighead, Cross, Lee, Monroe, Poinsett, St. Francis, and Woodruff Counties.

The elongated depression in Arkansas, Lonoke, and Prairie Counties has two areas that have changed in horizontal area or depth when compared to previous conditions of the aquifer. The area in Arkansas County in the southeastern half of the depression has not expanded horizontally from recent years, although the center of the depression has deepened. The area in Lonoke and Prairie Counties in the northwestern half of the depression has not expanded and water level in the deeper part of the depression has risen. In Lonoke and Prairie Counties in the northwestern half of the depression, the 90 -foot contour shown on the 2006 potentiometric-surface map is not shown on the 2008 potentiometric-surface map. Along the west side of Crowleys Ridge, the area enclosed by 140 -foot contour in Cross and Poinsett Counties has expanded further south into Cross County. The 130-foot contour in Poinsett County expanded north in 2008. The 130-foot contour is shown in Cross County, which was not evident in previous years. The 130-foot contour in St. Francis, Monroe, and Woodruff Counties in 2006 is not shown on the 2008 potentiometric-surface map.

A map showing the difference in water level was constructed using 595 differences in water levels measured in 585 wells during 2008 and 2004. The difference in measured water levels from 2004 to 2008 ranged from -20.6 feet to 25.9 feet, with a mean of -1.6 feet. The largest decline of -20.6 feet occurred in Randolph County and the largest rise of 25.9 feet occurred in Prairie County. Out of the 595 differences, 442 were declines ( 74.3 percent), 10 were no difference (values of $0.0 \mathrm{ft}$ ) (1.7 percent), and 143 were rises ( 24.0 percent). Five areas are dominated by declines that are west of Crowleys Ridge; in eastern Craighead County; in southern Mississippi and Crittenden Counties; in eastern Lonoke and western Prairie Counties; and in Arkansas, Ashley, Chicot, Desha, Drew, and Lincoln Counties.

Long-term water-level changes were evaluated using hydrographs from 173 wells in the Mississippi River Valley alluvial aquifer for the period 1984 to 2008. The mean annual rise or decline in water level for the entire study area was -0.38 feet per year ( $\mathrm{ft} / \mathrm{yr}$ ) with a range of -4.86 to $0.58 \mathrm{ft} / \mathrm{yr}$. Independence and White Counties are the only counties with a mean annual rise from 1984 to 2008. Mean annual declines between $-0.50 \mathrm{ft} / \mathrm{yr}$ and $0.00 \mathrm{ft} / \mathrm{yr}$ occurred in Arkansas, Chicot, Clay, Craighead, Crittenden, Drew, Greene, Jefferson, Mississippi, Monroe, Phillips, Poinsett, Prairie, Pulaski, Randolph, and Woodruff Counties. Mean annual declines between $-1.00 \mathrm{ft} / \mathrm{yr}$ and $-0.50 \mathrm{ft} / \mathrm{yr}$ occurred in Ashley, Desha, Jackson, Lee, Lincoln, and St. Francis Counties. Mean annual declines between $-1.50 \mathrm{ft} / \mathrm{yr}$ and $-1.00 \mathrm{ft} / \mathrm{yr}$ occurred in Cross and Lonoke Counties.

The analysis of long-term water-level changes in Arkansas, Lonoke, and Prairie Counties shows the elongation of the depression in these three counties. Arkansas and Prairie Counties have two different rates of annual decline for the two hydrographs shown for each county. Water levels in the two wells near the Arkansas and White Rivers have risen or declined at a slower rate than in the three wells in the center, northern, and western parts of the depression. These rates of water-level change indicate that this depression has expanded in an elongated direction north and west into Lonoke and Prairie Counties from 1984 to 2008. The depression west of Crowleys Ridge has five wells with hydrographs in or near the depression that can be used to characterize the rates of waterlevel change within this depression.

Water samples were collected from 60 wells completed in the Mississippi River Valley alluvial aquifer and measured onsite for specific conductance and temperature. Specific 
conductance ranged from 111 microsiemens per centimeter at 25 degrees Celsius $(\mu \mathrm{S} / \mathrm{cm})$ at a well in Lincoln County to $2,020 \mu \mathrm{S} / \mathrm{cm}$ at a well in Desha County. Specific conductance values equaled or exceeded $1,000 \mu \mathrm{S} / \mathrm{cm}$ in Arkansas, Chicot, Cross, Desha, Greene, and Lincoln Counties.

\section{Introduction}

The Mississippi Alluvial Plain (fig. 1) encompasses an area of approximately 32,000 square miles and includes parts of Arkansas and nearby States. Approximately 54 percent of the Mississippi Alluvial Plain covers the eastern one-third of Arkansas. The Mississippi River Valley alluvial aquifer (herein referred to as the alluvial aquifer) underlies the Mississippi Alluvial Plain in eastern Arkansas. Within Arkansas, the alluvial aquifer extends from the Missouri State line south to the Louisiana State line, and from the Mississippi River west to the Fall Line (the physiographic boundary between the Atlantic Plain and the Interior Highlands) and the Monticello Ridge (a topographic feature in southeastern Arkansas) (fig. 1).

Agricultural land use in eastern Arkansas has increased since 1900 with production consisting predominately of rice, soybeans, cotton, and, in recent years, aquaculture, all of which are highly dependent on the availability of water.

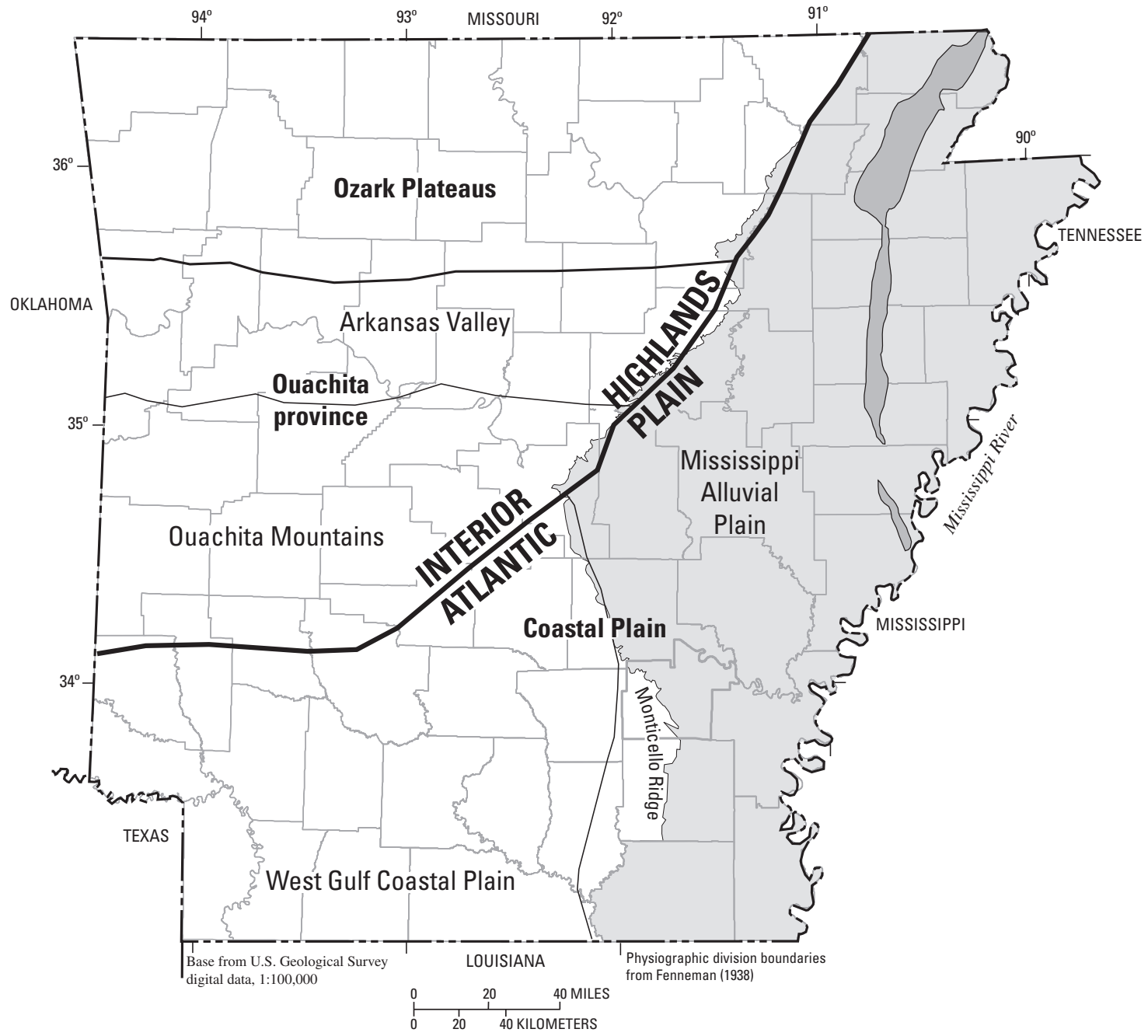

EXPLANATION

Study areas

$$
\begin{aligned}
& \text { Areal extent of Mississippi River } \\
& \text { Valley alluvial aquifer in eastern } \\
& \text { Arkansas }
\end{aligned}
$$

Crowleys Ridge
Physiographic division boundary

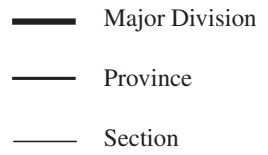

Figure 1. Location of study area. 
Eastern Arkansas receives sufficient precipitation to support these crops, receiving an average 46 to 54 inches of precipitation annually (Freiwald, 1984). However, during a critical part of the growing season from late spring through early summer, most precipitation in eastern Arkansas falls as rain from widely scattered thunderstorms, which may be insufficient for crop production.

The alluvial aquifer is increasingly relied upon for agriculture and aquaculture in eastern Arkansas. In 2005, estimated water withdrawals from the alluvial aquifer in Arkansas totaled about 7,252 million gallons per day (Mgal/d) (Holland, 2007); estimated withdrawals were about $1,063 \mathrm{Mgal} / \mathrm{d}$ in 1965 (fig. 2) (Halberg and Stephens, 1966). Starting in 1970 and continuing in 5-year intervals through 2000 , estimated water withdrawals from the alluvial aquifer in Arkansas totaled about 1,287 Mgal/d, 2,211 Mgal/d, 3,697 Mgal/d, 3,534 Mgal/d, 4,360 Mgal/d, 5,056 Mgal/d, and 6,585 Mgal/d (Halberg, 1972, 1977; Holland and Ludwig, 1981; Holland, $1987,1993,1999,2004)$. Since 1965, withdrawals from the alluvial aquifer have increased from about 1,063 Mgal/d to about 7,252 Mgal/d in 2005, an increase of about 582 percent. Withdrawals have more than doubled in the last 20 years, about a 105 percent increase since 1985 .

During the spring of 2008, the U.S. Geological Survey (USGS), in cooperation with the Arkansas Natural Resources Commission (ANRC) and the Arkansas Geological Survey, measured water levels in 319 wells completed in the alluvial aquifer in eastern Arkansas. The ANRC measured water levels in 39 wells and the U.S. Department of AgricultureNatural Resources Conservation Service (NRCS) measured water levels in 312 wells completed in the alluvial aquifer and provided these data to the ANRC. These data were made available to the USGS and were incorporated into the database used to develop a potentiometric-surface map of the alluvial aquifer for the spring of 2008. In the spring of 2008, a total of 670 water-level measurements were collected from 659 wells. Because the USGS and NRCS both measure water levels in 11 wells, a total of 670 measurements were made in 659 wells. During the summer of 2008, water samples from 60 wells completed in the alluvial aquifer were measured for specific conductance and temperature. These measurements provided information for a database of selected water-quality data for the alluvial aquifer.

This report describes the status and trends of water levels and selected water-quality conditions (specific conductance and temperature) in the alluvial aquifer. The report includes maps, long-term hydrographs, and data tables. Scheduled monitoring and evaluation of conditions in the alluvial aquifer provide information necessary for resource management.

The well-numbering system used in this report is based upon the locations of the wells according to the Federal land survey used in Arkansas. The component parts of a well number are the township number; the range number; the section number; three letters that indicate respectively, the quarter section, the quarter-quarter section, and the quarter-quarterquarter section in which the well is located; and a sequence

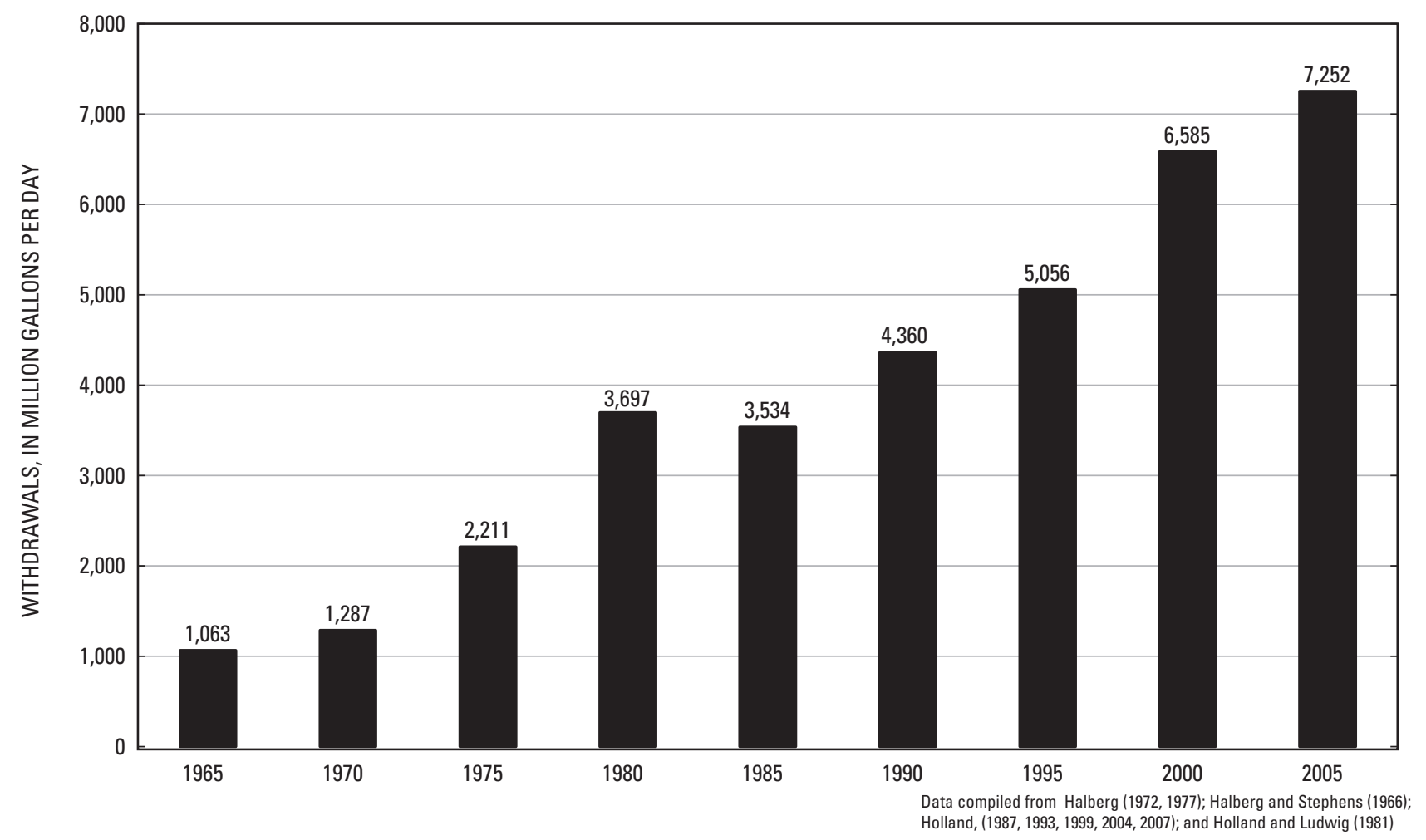

Figure 2. Estimated withdrawals from the Mississippi River Valley alluvial aquifer in Arkansas, 1965 to 2005. 
number of the well in the quarter-quarter-quarter section. The letters are assigned counterclockwise, beginning with " $\mathrm{A}$ " in the northeast quarter or quarter-quarter or quarter-quarterquarter section in which the well is located. For example, well 01S03W04BBD16 (fig. 3) is located in Township 1 South, Range 3 West, and in the southeast quarter of the northwest quarter of the northwest quarter of section 4 . This well is the 16 th well in this quarter-quarter-quarter section of section 4 from which data were collected.

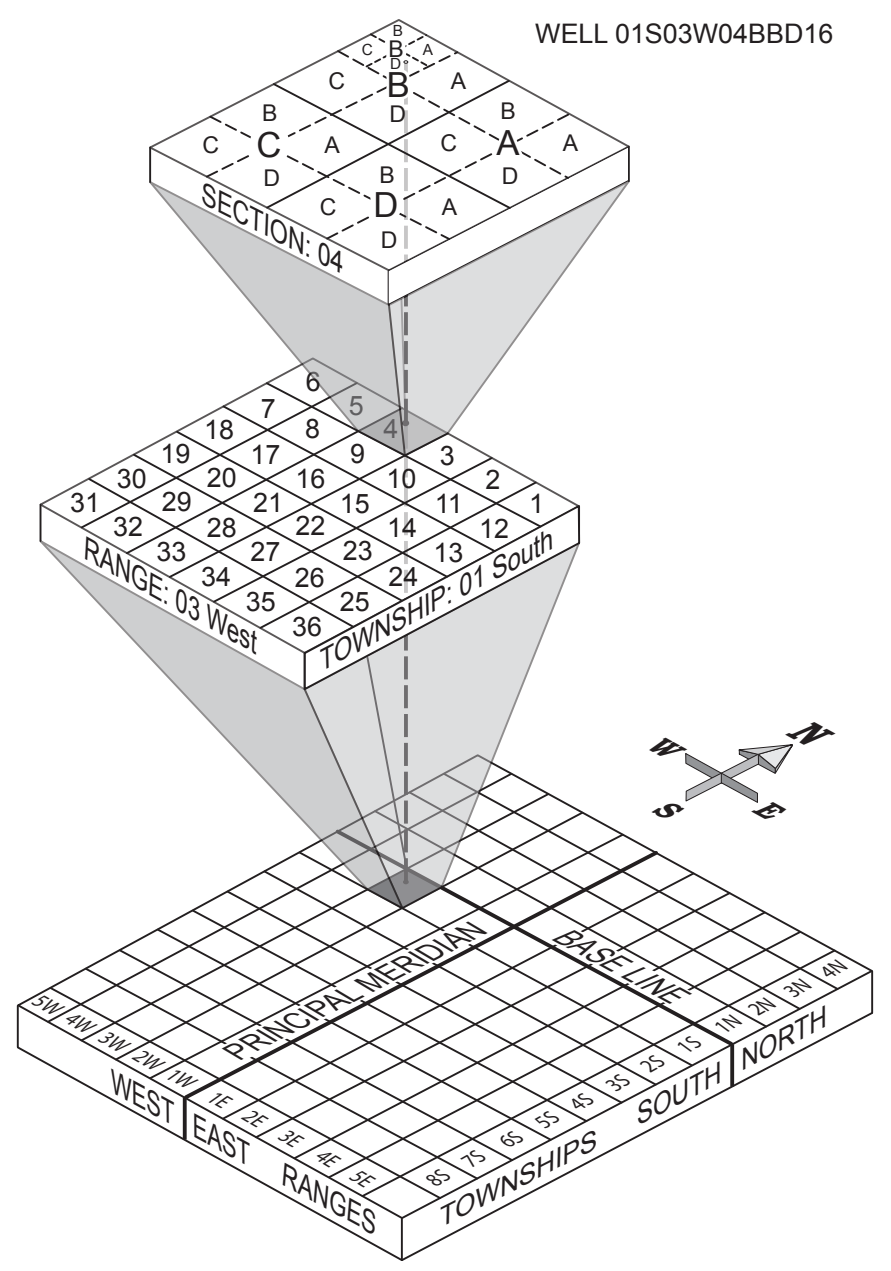

Figure 3. Well-numbering system.

\section{Methods}

Personnel from the USGS, the ANRC, and the NRCS measured water levels from February to May 2008 from wells completed in the alluvial aquifer. Measurements by USGS and ANRC personnel were made with steel or electric tapes graduated in hundredths of a foot, whereas measurements by NRCS personnel were in tenths of a foot or whole feet. The steel and electric tapes used by USGS and ANRC personnel were calibrated during January 2008 prior to collecting water-level measurements from wells. Calibration of steel and electric tapes was performed by comparing the field steel or electric tape to a standardized steel tape used only for calibration. Duplicate measurements were made by USGS and ANRC personnel in 11 wells for quality assurance.

Well locations were verified using Global Positioning System (GPS) receivers to acquire the horizontal-coordinate information, latitude and longitude, based on the North American Datum of 1983. The latitude and longitude of the wells in Arkansas were recorded from a GPS accurate to one-tenth of a second of latitude and longitude (approximately 10-20 feet (ft)). The latitude and longitude of the well location were transferred to a topographic map and the altitude of the well (National Geodetic Vertical Datum of 1929) was determined from the topographic contours at the well location on the map. Altitude is accurate to one-half of the contour interval (2.5 to 5 $\mathrm{ft}$ ) of the topographic map.

Two methods for calculating the annual rise or decline of water levels may be used. One method is to take the difference between the final and initial water levels and divide by the period of time. This method is determined from two measurements and calculated values are dependent solely on the final and initial water levels. A second method uses the linear regression of water levels over time to calculate the annual rise or decline in water level. Linear regression is more robust analysis because the trend line is determined using all the water-level measurements collected over the period of record instead of the two measurements used for the difference method. The slope, $\square$, of the trend line is the annual rise or decline in water level. The intercept, $\square 0$, would be the water level in the year 1900, the origin for the graph. This assumes the pumping rate was constant throughout the period of pumping. This condition is not commonly met or the data are not available to demonstrate that this has occurred. The predevelopment water level will not be discussed as this condition can not be demonstrated. The R2 term is the coefficient of determination, correlation coefficient, or the fraction of variance explained by the regression. The $\mathrm{R} 2$ value gives the proportions of the total variability that can be accounted by the independent variable (Helsel and Hirsch, 1992). Values of R2 can range from 0.00 to 1.00 . A high value of $\mathrm{R} 2$ can indicate a linear change in water level. A low value of R2 can indicate a sporadic change in water level.

Five assumptions are associated with linear repression: (1) $Y$ is linearly related to $X$, (2) data used to fit the linear regression are representative of the data of interest, (3) variance of the residuals is constant and does not depend on $X$ or on anything else, (4) the residuals are independent, and (5) the residuals are normally distributed. The assumption of a normal distribution is involved only when testing hypotheses, which requires the residuals from the regression equation to be normally distributed (Helsel and Hirsch, 1992).

Specific conductance was measured in groundwater from selected wells using specific-conductance meters with temperature compensation. Specific-conductance meters were calibrated twice daily by comparing the measurement of the specific-conductance meter of two specific conductance calibration standards. Most of the wells were irrigation 
wells sampled during pumping; specific conductance and temperature were monitored for 5 minutes or until the readings stabilized before values were recorded. For public supply and industrial wells, a minimum of three well volumes were purged and then specific conductance and temperature were monitored for 5 minutes or until the readings stabilized before values were recorded.

\section{Aquifer Description}

The alluvial aquifer comprises alluvial and terrace deposits of Quaternary age (Ackerman, 1996). Lithologically, the Quaternary alluvial and terrace deposits are similar, consisting of unconsolidated sediments that grade from gravel and coarse sand in the lower sections to silt and clay in the upper sections (Boswell and others, 1968). Because coarse sediments are contained in the lower sections of the alluvial and terrace deposits, the aquifer is capable of sustaining high-yielding wells (Ackerman, 1996). Finer sediments in the upper sections of the alluvial and terrace deposits form a confining unit above much of the aquifer. This confining unit is thin or has been completely removed by erosion in some areas, especially near large rivers within the study area (Gonthier and Mahon, 1993). Channel fill, point bar, and backswamp deposits associated with present or former channels of large rivers have produced abrupt changes in lithology and result in large spatial variations in the hydraulic properties of the aquifer (Joseph, 1999).

Sedimentary rocks and unconsolidated sediments of Tertiary age or older underlie the alluvial aquifer and have been modified by geologic processes into an undulating surface (Mahon and Poynter, 1993). In most areas, these rocks and sediments are less permeable than the overlying alluvial and terrace deposits of Quaternary age and form the confining unit below the alluvial aquifer (Boswell and others, 1968).

In the northern half of the study area, the alluvial and terrace deposits of Quaternary age are separated by Crowleys Ridge (fig. 1), an erosional remnant of deposits of Tertiary age trending north-south from the Missouri-Arkansas border. Crowleys Ridge is a prominent topographic feature on the otherwise low-relief surface of the Mississippi Alluvial Plain and forms a physical barrier to groundwater flow in the alluvial aquifer.

\section{Water Levels}

Water-level measurements collected in wells completed in the alluvial aquifer (appendix 1) were used to produce a regional potentiometric-surface map (plate 1). The water-level altitudes for the potentiometric-surface map were calculated by subtracting the depth-to-water measurement from the land-surface altitude, then rounding to the nearest foot. A difference in water-level map from 2004 to 2008 (plate 2) was produced by subtracting water-level measurements in 2008 from measurements in 2004. Data from wells that have waterlevel measurements with a minimum 25-year period of record were used to produce hydrographs shown in figure 4 . The water-level changes shown in the hydrographs indicate longterm changes in hydrologic conditions. Long-term water-level changes shown by many of the hydrographs reflect the development of cones of depression in the potentiometric surface.

\section{Potentiometric Surface}

The potentiometric-surface map (plate 1) shows the altitude at which water would have stood in tightly cased wells completed in the alluvial aquifer. The map on plate 1 is based on 670 water-level measurements ( 319 by USGS, 39 by ANRC, and 312 by NRCS) made in 659 wells during the spring of 2008 (appendix 1). Eleven wells were measured by both the USGS and NRCS. The potentiometric surface was mapped using the altitude of the water levels measured in the wells and is represented on the map by contours that connect points of equal altitude. The general direction of groundwater flow is perpendicular to the contours in the direction of decreasing potentiometric-surface altitude.

Groundwater levels are affected by groundwater withdrawals within the study area, often resulting in depressions. The depressions or other areas of reduced water level are shaded on plate 1. In 2008, the lowest water-level altitude was $69 \mathrm{ft}$ in the center of Arkansas County. The highest water-level altitude was $288 \mathrm{ft}$ in northeastern Clay County on the western side of Crowleys Ridge.

Previous reports described three large depressions in the alluvial aquifer potentiometric surface (Stanton and others, 1998; Joseph, 1999; Schrader, 2001; Reed, 2004; Schrader, 2006, 2008). A large, elongated area of depression extended across Arkansas, Lonoke, and Prairie Counties. Two shallower depressions were documented in Lee, Monroe, St. Francis, and Woodruff Counties, and in Craighead, Cross, and Poinsett Counties that have merged for a single depression.

The elongated depression in Arkansas, Lonoke, and Prairie Counties has two areas that have changed in horizontal area or depth when compared to previous conditions of the aquifer. The area in Arkansas County within the southeastern half of the depression has not expanded horizontally during recent years, although the center of the depression has deepened. The Arkansas and White Rivers that bound Arkansas County on the southwestern and eastern county lines are hydrologically connected and provide recharge to the alluvial aquifer. A comparison of measured water-level altitudes from 1998 through 2008 at the location of the lowest measured water level in the depression in central Arkansas County indicates water levels rose $8 \mathrm{ft}$ from 1998 (78 ft) to 2002 (86 ft), declined $10 \mathrm{ft}$ from 2002 to 2004 (76 ft), no change from 2004 to 2006 (76 ft), and declined $7 \mathrm{ft}$ from 2006 to 2008 (69 ft).

The area in Lonoke and Prairie Counties in the northwestern half of the depression has not expanded, and water level in the deeper part of the depression has risen. The extent 
of the 130-foot contour is generally the same in both the 2006 and 2008 potentiometric-surface maps. The 90 -foot contour shown on the 2006 potentiometric surface (Schrader, 2008) is not shown on the 2008 potentiometric-surface map. The lowest measured water-level altitudes in Lonoke County show a slow rise from $88 \mathrm{ft}$ in 2002 (Reed, 2004) to $92 \mathrm{ft}$ in 2008.

Along the western side of Crowleys Ridge, two previously documented areas of depression expanded and coalesced into a single depression by 2002 (Reed, 2004). The 2008 potentiometric-surface map shows little change in the area of this depression, although the deeper areas within the depression have changed. The 2008 map shows that the area enclosed by the 150 -foot contour is similar in area to the areas in the 2004 and 2006 potentiometric-surface maps. The area enclosed by 140 -foot contour in Lee, Monroe, St. Francis, and Woodruff Counties is similar in area to the area in the 2006 potentiometric-surface map. The area enclosed by 140 -foot contour in Cross and Poinsett Counties has expanded further south into Cross County since 2006. The 130-foot contour in St. Francis, Monroe, and Woodruff Counties in 2006 is not shown in the 2008 potentiometric-surface map. In 2008, water levels were not measured in this area; in previous years, this area was the deepest part of the depression. The lack of data points in this area may have caused the 130-foot contour not to be shown in the 2008 potentiometric-surface map. The area enclosed by the 130 -foot contour in eastern Monroe County is similar in area to the 2006 potentiometric-surface map. The 130-foot contour in Poinsett County has expanded north in 2008. The 130-foot contour is shown in Cross County, which was not evident in previous years.

In central Drew County, near the western boundary of the study area, a cone of depression was first noted in the 2002 potentiometric-surface map (Reed, 2004). The area enclosed by the 130-foot contour expanded in 2004, and the water level in the center of the cone declined by $8 \mathrm{ft}$ to $118 \mathrm{ft}$ (Schrader, 2006). In 2006, the area decreased to approximately the same area as in the 2002 potentiometric-surface map (Schrader, 2008). In 2008, the area was approximately the same area as in 2006.

Three areas of reduced water level were noted by Schrader (2001) in southeastern Arkansas - one in eastern Lincoln County, a second that extends from southern Desha County into northern Chicot County, and a third that extends from western Chicot County into eastern Ashley County. The area of reduced water level in southern Desha and northern Chicot Counties was first evident in the 1998 potentiometric surface (Joseph, 1999) and had expanded horizontally and vertically by 2000 (Schrader, 2001). This area expanded southward by 2002 but had not appreciably deepened. The potentiometric surface in this area showed little change between 2002 and 2004 (Schrader, 2006). In the 2006 potentiometric surface, this area had expanded further north into Desha County and south into Chicot County. In the 2008 potentiometric surface, this area is about the same size as in 2006. The water levels in this area do not have a trend, where some water levels have risen and some have declined 1 to $2 \mathrm{ft}$ since 2006. The areas in eastern Lincoln County and in western Chicot and eastern Ashley Counties were not evident in 1996 and 1998. The area in eastern Lincoln County expanded into northwestern Desha County and continued to deepen through 2004, with an altitude of $118 \mathrm{ft}$ (Schrader, 2006) at its deepest point. In 2006, this area had expanded westward in Lincoln County, with a measured altitude of $119 \mathrm{ft}$ at its deepest point. In 2008, the deepest measured altitude was $121 \mathrm{ft}$. The area in western Chicot and eastern Ashley Counties showed little change in the 2004 potentiometric surface from the 2002 potentiometric surface (Schrader, 2006). From 2004 to 2008, the depth of this area has not increased and is approximately the same area.

A potentiometric area of reduced water level in Greene County noted in 1998 by Joseph (1999), and in 2000 by Schrader (2001), deepened by 2002 (Reed, 2004). This area contracted in 2004 (Schrader, 2006). In 2006, this area had expanded and deepened (Schrader, 2008). In 2008, the water level had risen $6 \mathrm{ft}$ and the area had declined to about a quarter of the area shown in 2006.

Six other depressions are shown in the 2008 potentiometric-surface map, three that were first shown in the 2006 potentiometric surface and two that have not been previously shown. A depression south of the Prairie and White County line is enclosed by a 130 -foot contour. In the 2004 potentiometric surface, the 130 -foot contour line shows a small lobe extending northeast from the larger depression (Schrader, 2006). In the 2008 potentiometric surface, the 130 -foot contour line is approximately oval shaped and separate from the larger, elongated depression in Arkansas, Lonoke, and Prairie Counties. A second depression at the Craighead and Mississippi County line is enclosed by a 220 -foot contour. Two small depressions are located in northeastern and central St. Francis County. The northeastern depression is enclosed by a 160foot contour, first shown on the 2006 map (Schrader, 2008), and the central depression is on the western side of Crowleys Ridge, enclosed by a 150 -foot contour, first shown on the 2002 map (Reed, 2004). A depression is located in eastern Randolph County, enclosed by a 260 -foot contour, which has not been shown on previous potentiometric-surface maps. In north-central Ashley County a small depression is enclosed by a 100 -foot contour that has not been shown on previous potentiometric-surface maps, but additional data from waterlevel measurements were available in 2008. Three depressions in Desha, Jefferson, and Lee Counties were shown on the 2006 potentiometric surface (Schrader, 2008). The depression in Jefferson County is not shown on the 2008 potentiometric surface. Continued monitoring of the potentiometric surface will determine if these depressions are the result of short-term variations or long-term changes in the hydrologic conditions in the alluvial aquifer.

The regional direction of groundwater flow is generally to the south and east except where flow is affected by groundwater withdrawals; however, the flow direction is affected over substantial areas by depressions (plate 1). West of Crowleys Ridge, depressions in Arkansas, Lonoke, and Prairie Counties capture groundwater flow from all directions. The flow along 
large sections of the Arkansas, Mississippi, and White Rivers is away from the rivers. East of Crowleys Ridge water flows from north to south along Crowleys Ridge and northeast to southwest along the Mississippi River. South of the Arkansas River the flow is towards the southeast, except in northwestern Desha County where flow is towards the area of reduced water level.

\section{Water-Level Difference from 2004 to 2008}

A map showing the difference in water level (plate 2) was constructed using 595 differences in water levels measured in 585 wells during 2008 (appendix 2) and 2004 (Schrader, 2006). Differences in water level were calculated by subtracting the 2008 depth-to-water measurement from the 2004 depth-to-water measurement. Positive values indicate a rise and negative values indicate a decline in water level. Rises in water level are indicated on plate 2 with blue triangles pointing upward; declines in water level are indicated with red triangles pointing downward. The triangles are scaled to the value of rise or decline.

The difference in measured water levels from 2004 to 2008 ranged from $-20.6 \mathrm{ft}$ to $25.9 \mathrm{ft}$, with a mean of $-1.6 \mathrm{ft}$. The largest decline of $-20.6 \mathrm{ft}$ occurred in Randolph County and the largest rise of $25.9 \mathrm{ft}$ occurred in Prairie County. Out of the 596 differences on plate 2, 442 were declines (74.3 percent), 10 were no difference (values of $0.0 \mathrm{ft}$ ) ( 1.7 percent), and 143 were rises (24.0 percent). Five areas are documented by declines that are west of Crowleys Ridge; in eastern Craighead County; in southern Mississippi and Crittenden Counties; in eastern Lonoke and western Prairie Counties; and in Arkansas, Ashley, Chicot, Desha, Drew, and Lincoln Counties.

Six areas are dominated by rises in measured water levels. The largest area is in northern Prairie, White, and western Woodruff Counties adjacent to the White River. Two areas are in Jefferson County and northern Mississippi County. Three small areas with water-level rises are located in western Greene and Randolph Counties, Independence and northwestern Jackson Counties, and eastern Greene County.

\section{Long-Term Water-Level Changes}

Long-term water-level changes were evaluated using hydrographs from 173 wells in the alluvial aquifer for the period 1984 to 2008. Linear regression was used to calculate the trend in water-level change for each well for this period. The slope of the trend line represents the mean annual rise or decline in water level during the 25 -year period. Negative values denote a decline in water level. The minimum 25-year period is used to show long-term trends not dominated by short-term variations in climate and localized pumping rates on water levels in a single well. The hydrographs were grouped by county. The number of wells, the range of values for the annual rise or decline in water level, the mean, the median, and the correlation coefficient (R2) for each county are listed in table 1 . The mean annual rise or decline in water level for the entire study area was -0.38 feet per year $(\mathrm{ft} / \mathrm{yr})$ with a range of -4.86 to $0.58 \mathrm{ft} / \mathrm{yr}$. Selected hydrographs are shown in figure 4 (wells A-CC, plate 1).

Long-term water-level changes vary substantially across the study area. Independence and White Counties are the only counties with a mean annual rise from 1984 to 2008. The rise in Independence County is determined from the data of one well. Mean annual declines between $-0.50 \mathrm{ft} / \mathrm{yr}$ and $0.00 \mathrm{ft} /$ yr occurred in Arkansas, Chicot, Clay, Craighead, Crittenden, Drew, Greene, Jefferson, Mississippi, Monroe, Phillips, Poinsett, Prairie, Pulaski, Randolph, and Woodruff Counties. Mean annual declines between $-1.00 \mathrm{ft} / \mathrm{yr}$ and $-0.50 \mathrm{ft} / \mathrm{yr}$ occurred in Ashley, Desha, Jackson, Lee, Lincoln, and St. Francis Counties. Mean annual declines between $-1.50 \mathrm{ft} / \mathrm{yr}$ and $-1.00 \mathrm{ft} / \mathrm{yr}$ occurred in Cross and Lonoke Counties.

The analysis of long-term water-level changes (19842008) in Arkansas, Lonoke, and Prairie Counties shows the elongation of the depression in these three counties. Arkansas and Prairie Counties have two different rates of annual decline for the two hydrographs shown for each county. In Arkansas County, well 04S03W32BCB1 (fig. 4A) shows a mean annual water-level decline of about $0.95 \mathrm{ft} / \mathrm{yr}$ since 1984 . Well $\mathrm{A}$ is located near the center of the depression in Arkansas County and generally shows a water-level decline during the 70-year period of record. Well 07S04W01DDD1 (fig. 4B) has a mean annual water-level rise of about $0.07 \mathrm{ft} / \mathrm{yr}$ since 1984 . Well $\mathrm{B}$ is located near the Arkansas River and shows a relatively stable water level for the 79-year period. The water level in the Arkansas River is maintained by a lock and dam system and can be a source of water for the alluvial aquifer in southern and western Arkansas County. In Prairie County, well 02N04W32CCB1 (fig. 4X) is near the depression and the White River. This well has a mean annual water-level decline of about $0.37 \mathrm{ft} / \mathrm{yr}$ since 1984. Well 04N05W07CDC1 (fig. 4W) is located in the central part of Prairie County and has a mean annual decline of about $0.66 \mathrm{ft} / \mathrm{yr}$ since 1984 . These two hydrographs show that the rate of decline in the northern part of the depression is nearly twice the rate of decline near the White River. Well 02S07W10CCB1 (fig. 4Q), near the western edge of the depression in Lonoke County, shows a mean annual water-level decline of about 0.88 $\mathrm{ft} / \mathrm{yr}$ since 1984 and shows a nearly continuous water-level decline during the 51 years of record. In Arkansas, Lonoke, and Prairie Counties, water levels in the two wells near the Arkansas and White Rivers have risen or declined at a slower rate than in the three wells in the center, northern, and western part of the depression. These rates of water-level change indicate that this depression has expanded in an elongated direction north and west into Lonoke and Prairie Counties from 1984 to 2008 . Water-level declines in neighboring counties are further evidence of the expansion of the depression centered in Arkansas, Lonoke, and Prairie Counties. In Jefferson County, well 03S08W24BBC1 (fig. 4N) has a mean annual water-level decline of about $0.69 \mathrm{ft} / \mathrm{yr}$ during the period 1984 to 2008 . Long-term declines in this outlying well indicates that the depression is expanding. 


\section{Water Levels and Selected Water-Quality Conditions in the Mississippi River Valley Alluvial Aquifer}

The depression west of Crowleys Ridge has six wells with hydrographs in or near the depression that can be used to characterize the rates of water-level change within the depression. Wells 07N01E05CDA1 (fig. 4H) in Cross County, 03N01W20ABA1 (fig. 4S) in Monroe County, 11N02E26AAB1 (fig. 4U) in Poinsett County, and 04N01W28CDD1 (fig. 4AA) in St. Francis County are in the depression and have mean annual declines of about $0.99 \mathrm{ft} /$ yr, $0.69 \mathrm{ft} / \mathrm{yr}, 1.28 \mathrm{ft} / \mathrm{yr}$, and $0.66 \mathrm{ft} / \mathrm{yr}$, respectively, since 1984. For the period of record, well H in Cross County, since 1946, and well U in Poinsett County, since 1958, have the largest declines in the alluvial aquifer, about $51.5 \mathrm{ft}$ and 58.6 $\mathrm{ft}$, respectively. Wells 14N02E18BDD1 (fig. 4F) in Craighead County and 02N01E23BAA2 (fig. 4O) in Lee County near the edge of the depression have mean annual water-level declines of about $0.99 \mathrm{ft} / \mathrm{yr}$ and $0.55 \mathrm{ft} / \mathrm{yr}$, respectively, since 1984 .

Table 1. Range, mean, median, and correlation coefficient of annual rise-decline in water level by county for wells in the Mississippi River Valley alluvial aquifer, 1984-2008.

[Annual rise or decline in water level for each well is calculated using linear regression; negative value indicates decline; positive value indicates rise. $\mathrm{R}^{2}$ is correlation coefficient]

\begin{tabular}{|c|c|c|c|c|c|}
\hline County & $\begin{array}{l}\text { Number } \\
\text { of wells }\end{array}$ & $\begin{array}{c}\text { Range of annual rise- } \\
\text { decline in } \\
\text { water level } \\
\text { (feet/year) }\end{array}$ & $\begin{array}{l}\text { Mean annual } \\
\text { rise-decline in } \\
\text { water level } \\
\text { (feet/year) }\end{array}$ & $\begin{array}{c}\text { Median annual } \\
\text { rise-decline in } \\
\text { water level } \\
\text { (feet/year) }\end{array}$ & $\begin{array}{l}\text { Range of } R^{2} \text { values } \\
\text { for regression line }\end{array}$ \\
\hline Arkansas & 30 & -0.95 to 0.58 & -0.19 & -0.24 & 0.01 to 1.00 \\
\hline Ashley & 10 & -4.86 to 0.11 & -0.69 & -0.29 & 0.01 to 0.77 \\
\hline Chicot & 7 & -1.06 to 0.00 & -0.39 & -0.37 & 0.00 to 0.89 \\
\hline Clay & 7 & -0.99 to 0.00 & -0.37 & -0.37 & 0.00 to 0.97 \\
\hline Craighead & 6 & -0.99 to 0.00 & -0.41 & -0.27 & 0.00 to 0.95 \\
\hline Crittenden & 5 & -0.69 to -0.18 & -0.41 & -0.37 & 0.40 to 0.95 \\
\hline Cross & 7 & -3.18 to -0.26 & -1.15 & -1.06 & 0.25 to 0.98 \\
\hline Desha & 6 & -1.13 to -0.11 & -0.69 & -0.75 & 0.37 to 0.97 \\
\hline Drew & 4 & -0.37 to -0.18 & -0.30 & -0.33 & 0.72 to 0.81 \\
\hline Greene & 4 & -0.80 to -0.15 & -0.48 & -0.49 & 0.20 to 0.88 \\
\hline Independence & 1 & 0.18 & 0.18 & 0.18 & 0.06 \\
\hline Jackson & 5 & -0.91 to -0.37 & -0.72 & -0.77 & 0.86 to 0.98 \\
\hline Jefferson & 7 & -0.69 to 0.00 & -0.31 & -0.26 & 0.00 to 0.93 \\
\hline Lee & 5 & -0.69 to -0.37 & -0.58 & -0.62 & 0.43 to 0.95 \\
\hline Lincoln & 4 & -1.02 to -0.44 & -0.77 & -0.80 & 0.80 to 0.92 \\
\hline Lonoke & 8 & -2.74 to -0.47 & -1.21 & -0.93 & 0.41 to 0.98 \\
\hline Mississippi & 8 & -0.33 to 0.02 & -0.09 & -0.07 & 0.00 to 0.68 \\
\hline Monroe & 9 & -0.69 to -0.04 & -0.34 & -0.33 & 0.07 to 0.94 \\
\hline Phillips & 3 & -0.33 to -0.04 & -0.22 & -0.29 & 0.04 to 0.80 \\
\hline Poinsett & 5 & -1.28 to 0.11 & -0.38 & -0.18 & 0.15 to 0.95 \\
\hline Prairie & 11 & -0.95 to 0.22 & -0.38 & -0.33 & 0.02 to 0.94 \\
\hline Pulaski & 1 & -0.29 & -0.29 & -0.29 & 0.45 \\
\hline Randolph & 2 & -0.26 to -0.07 & -0.16 & -0.16 & 0.02 to 0.78 \\
\hline St Francis & 7 & -0.95 to -0.03 & -0.58 & -0.69 & 0.10 to 0.98 \\
\hline White & 6 & -0.37 to 0.26 & 0.11 & 0.18 & 0.26 to 0.62 \\
\hline Woodruff & 5 & -0.66 to 0.01 & -0.19 & -0.15 & 0.03 to 0.90 \\
\hline
\end{tabular}




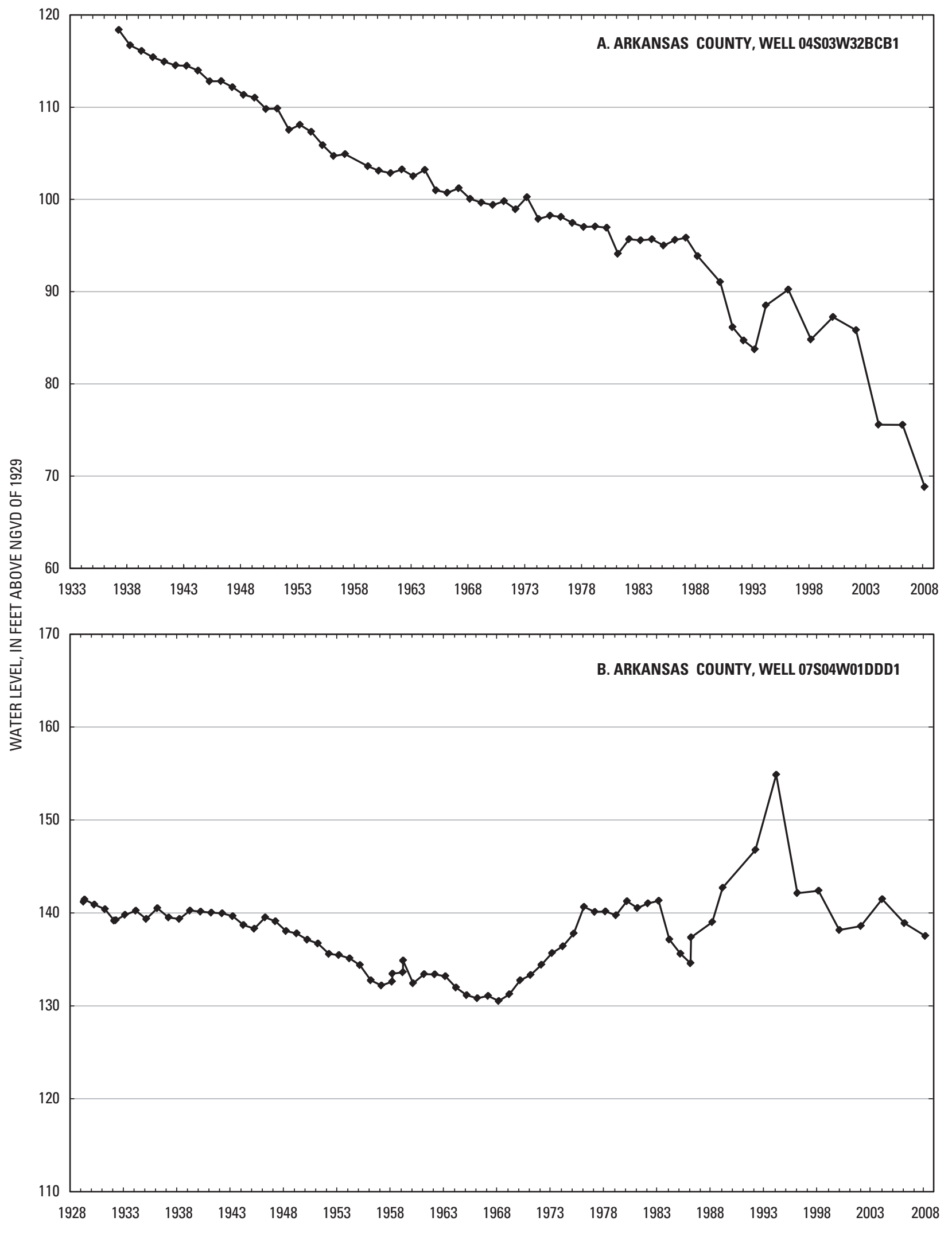

Figure 4. Water-level hydrographs (A to CC) for selected wells in the Mississippi River Valley alluvial aquifer. 


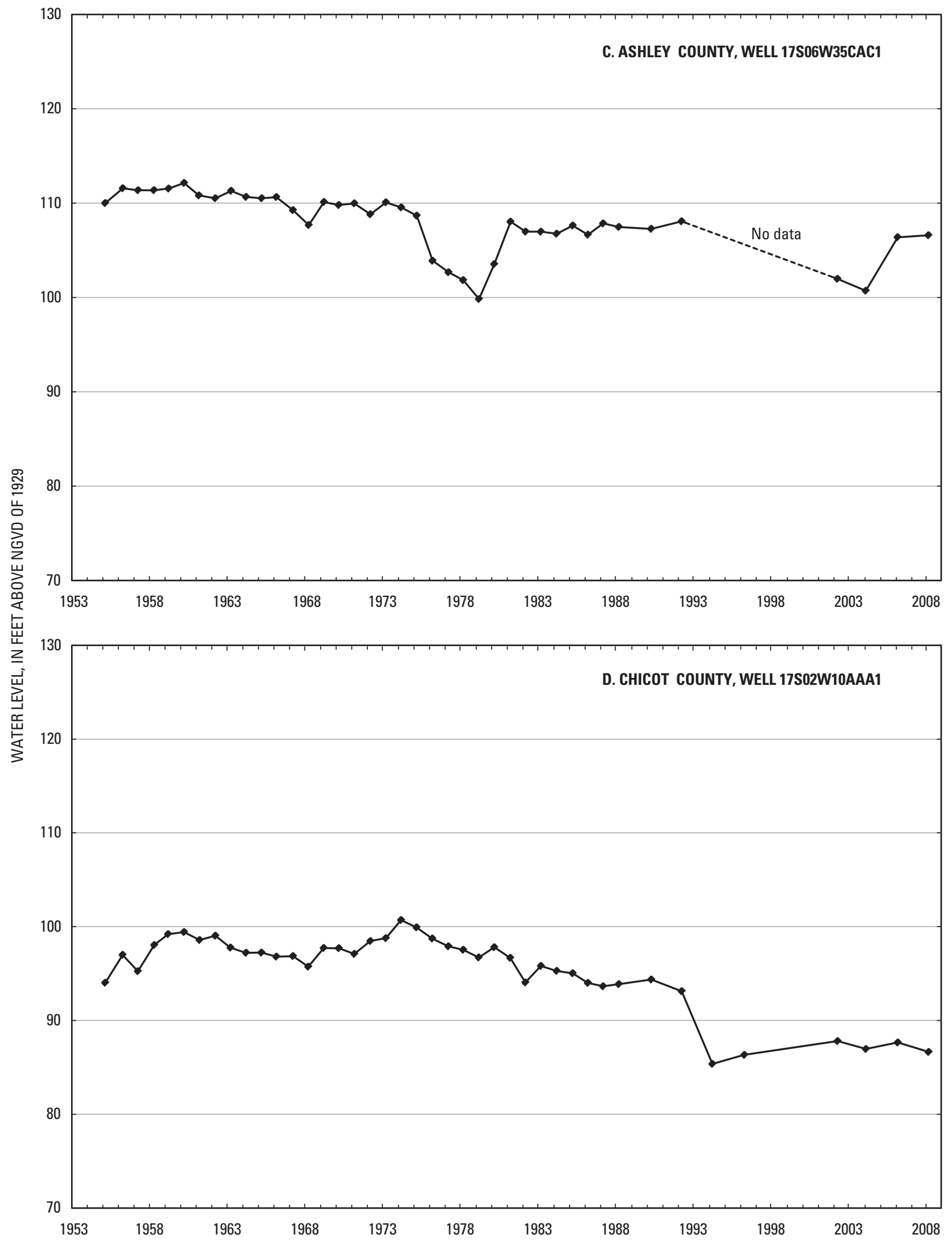

Figure 4. Water-level hydrographs (A to CC) for selected wells in the Mississippi River Valley alluvial aquifer.—Continued 


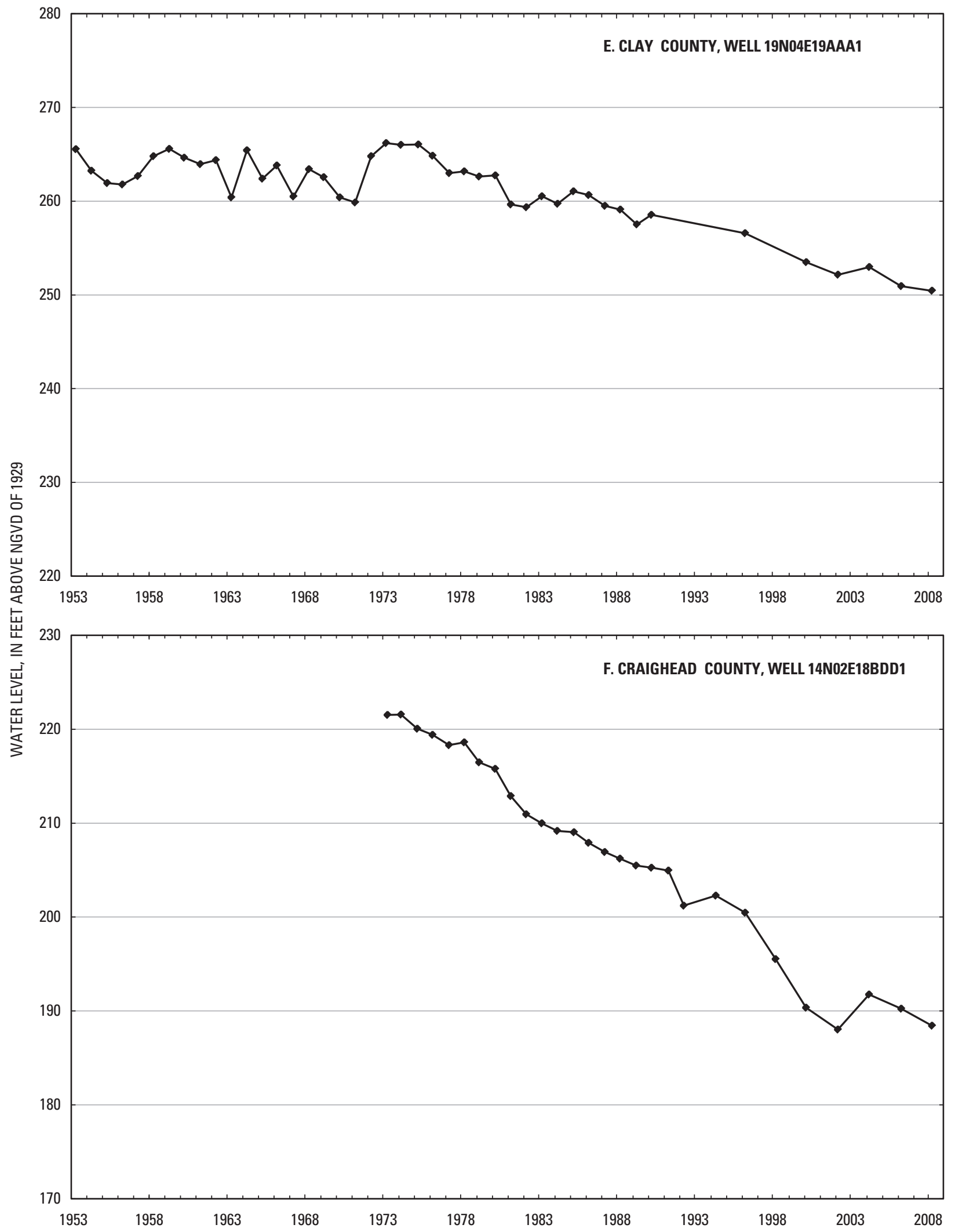

Figure 4. Water-level hydrographs (A to CC) for selected wells in the Mississippi River Valley alluvial aquifer-Continued 


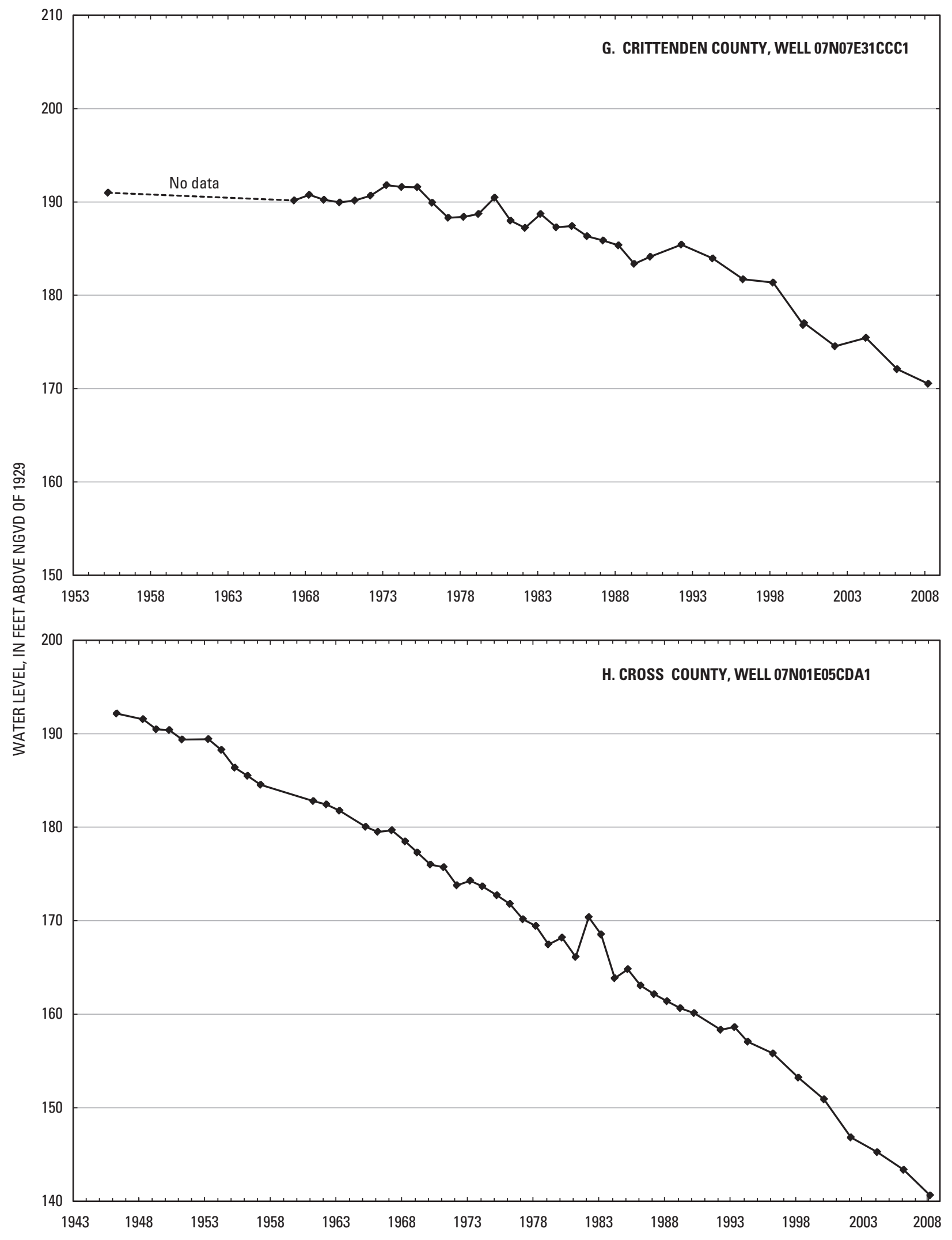

Figure 4. Water-level hydrographs (A to CC) for selected wells in the Mississippi River Valley alluvial aquifer-Continued 


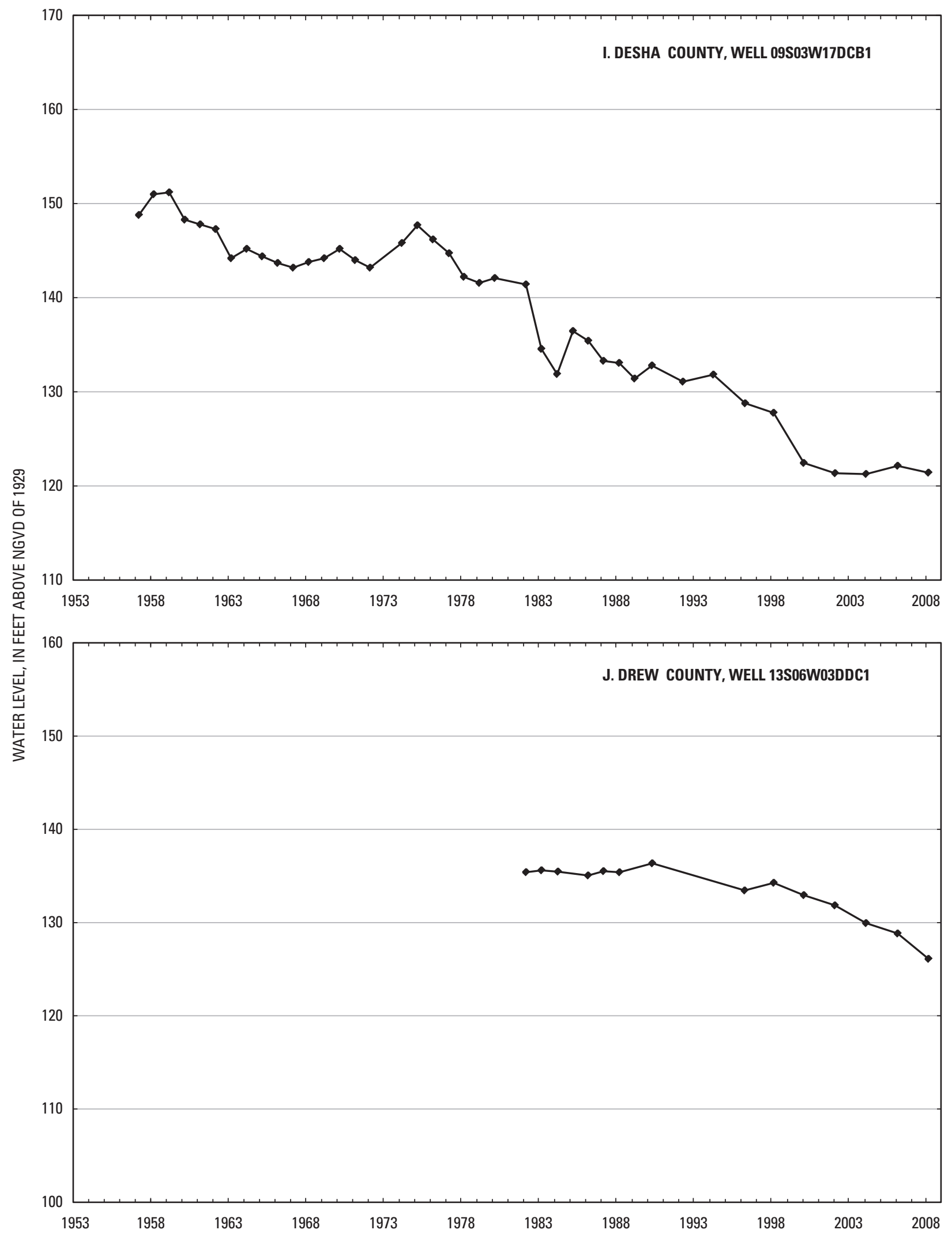

Figure 4. Water-level hydrographs (A to CC) for selected wells in the Mississippi River Valley alluvial aquifer-CContinued 


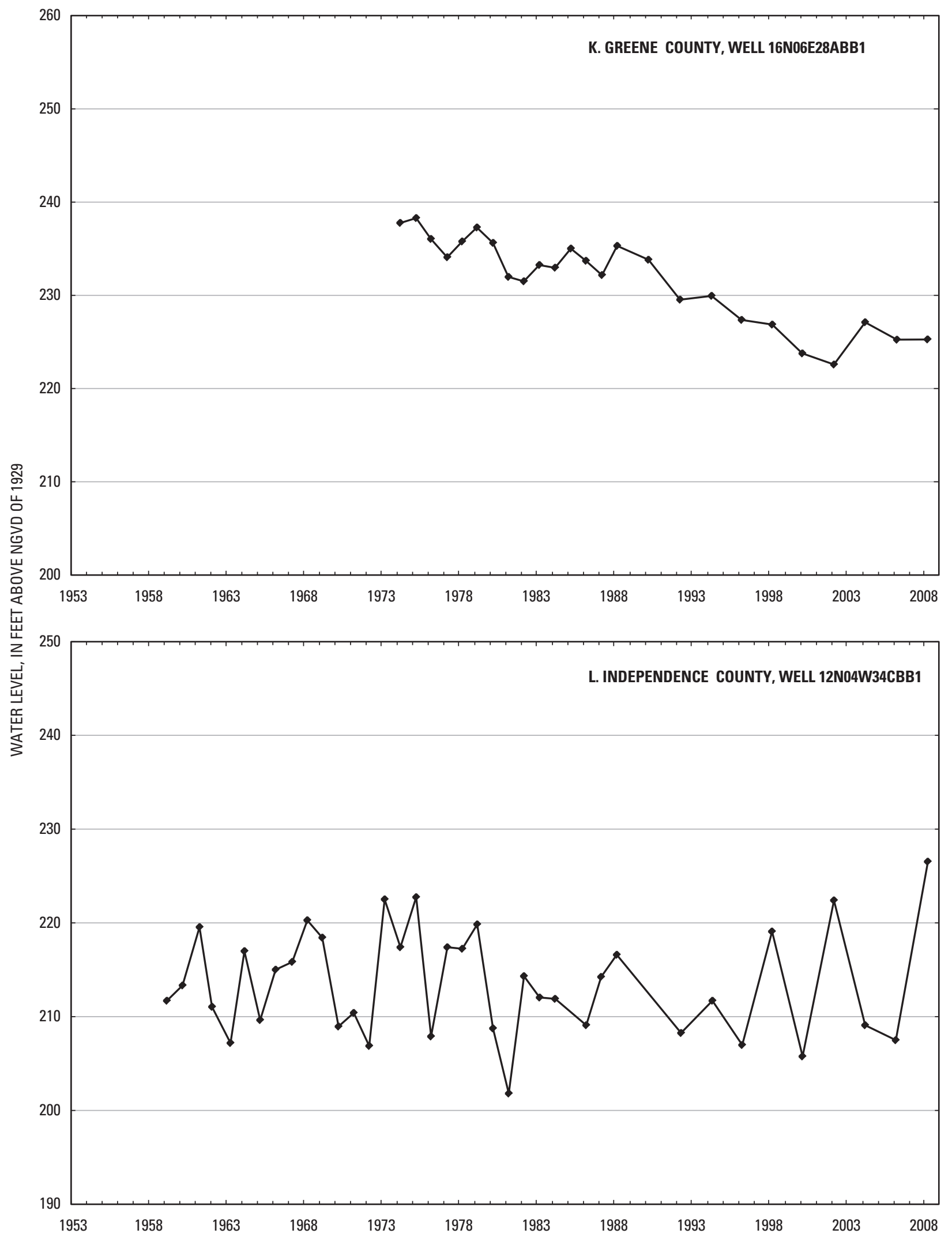

Figure 4. Water-level hydrographs (A to CC) for selected wells in the Mississippi River Valley alluvial aquifer.—Continued 


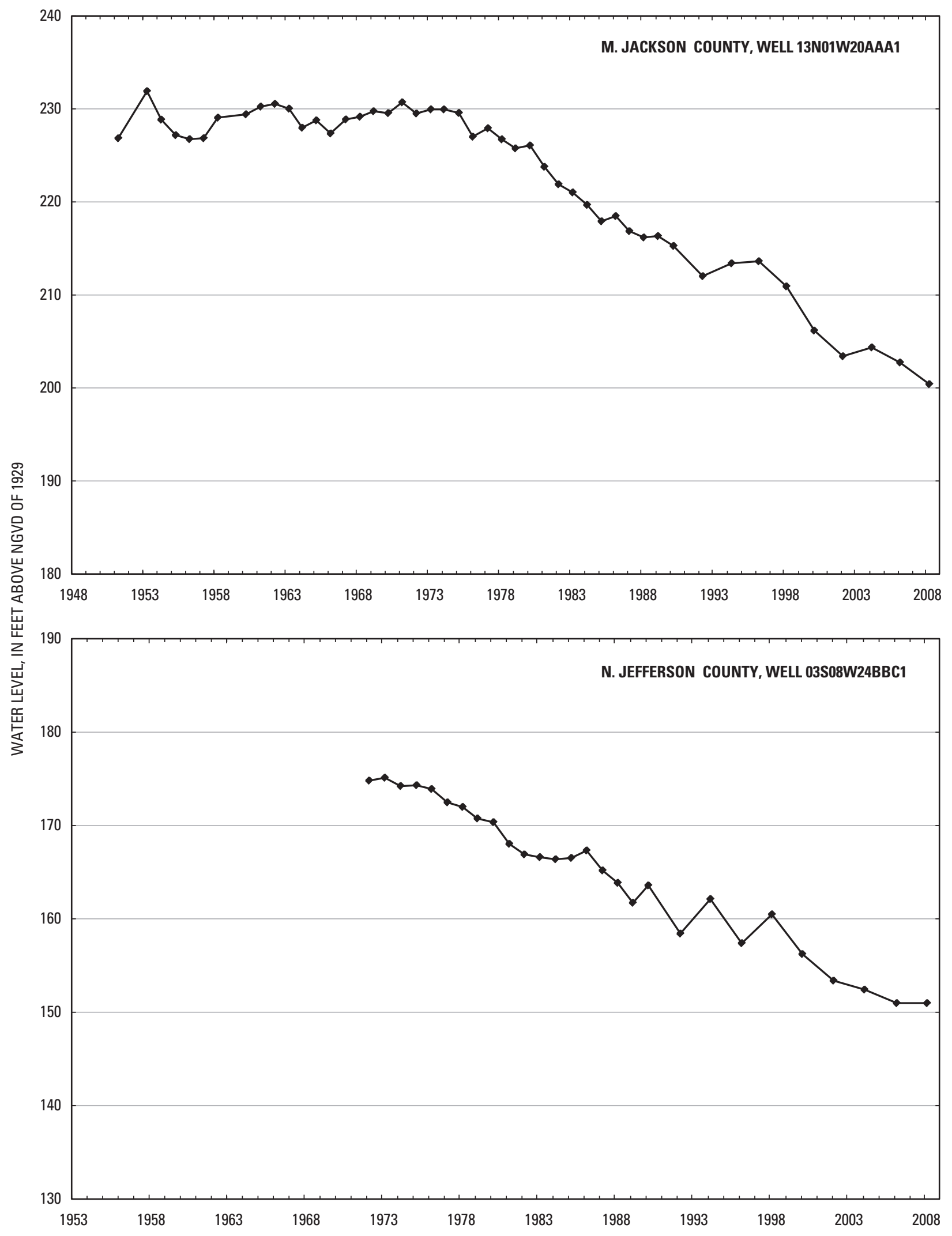

Figure 4. Water-level hydrographs (A to CC) for selected wells in the Mississippi River Valley alluvial aquifer-Continued 


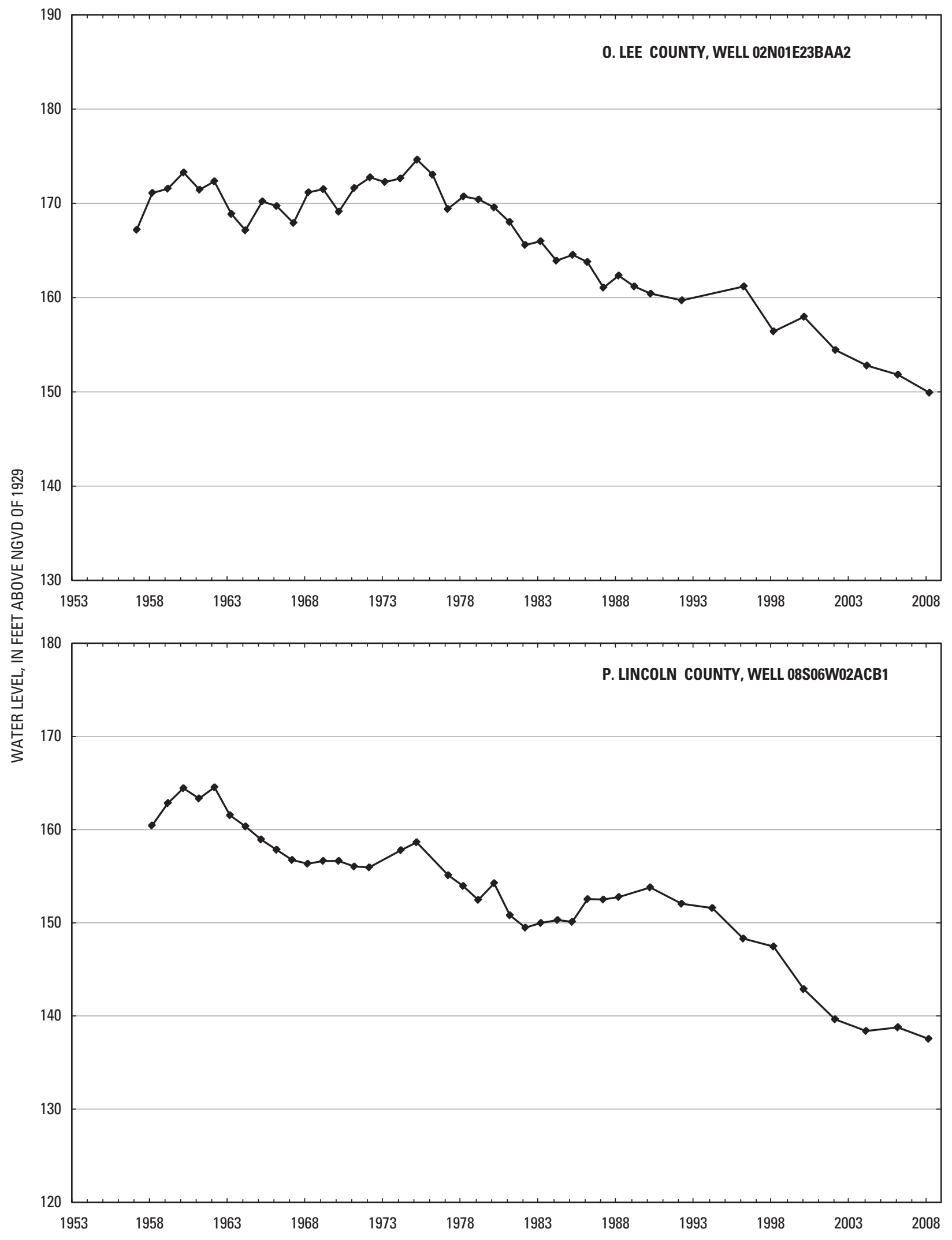

Figure 4. Water-level hydrographs (A to CC) for selected wells in the Mississippi River Valley alluvial aquifer.—Continued 


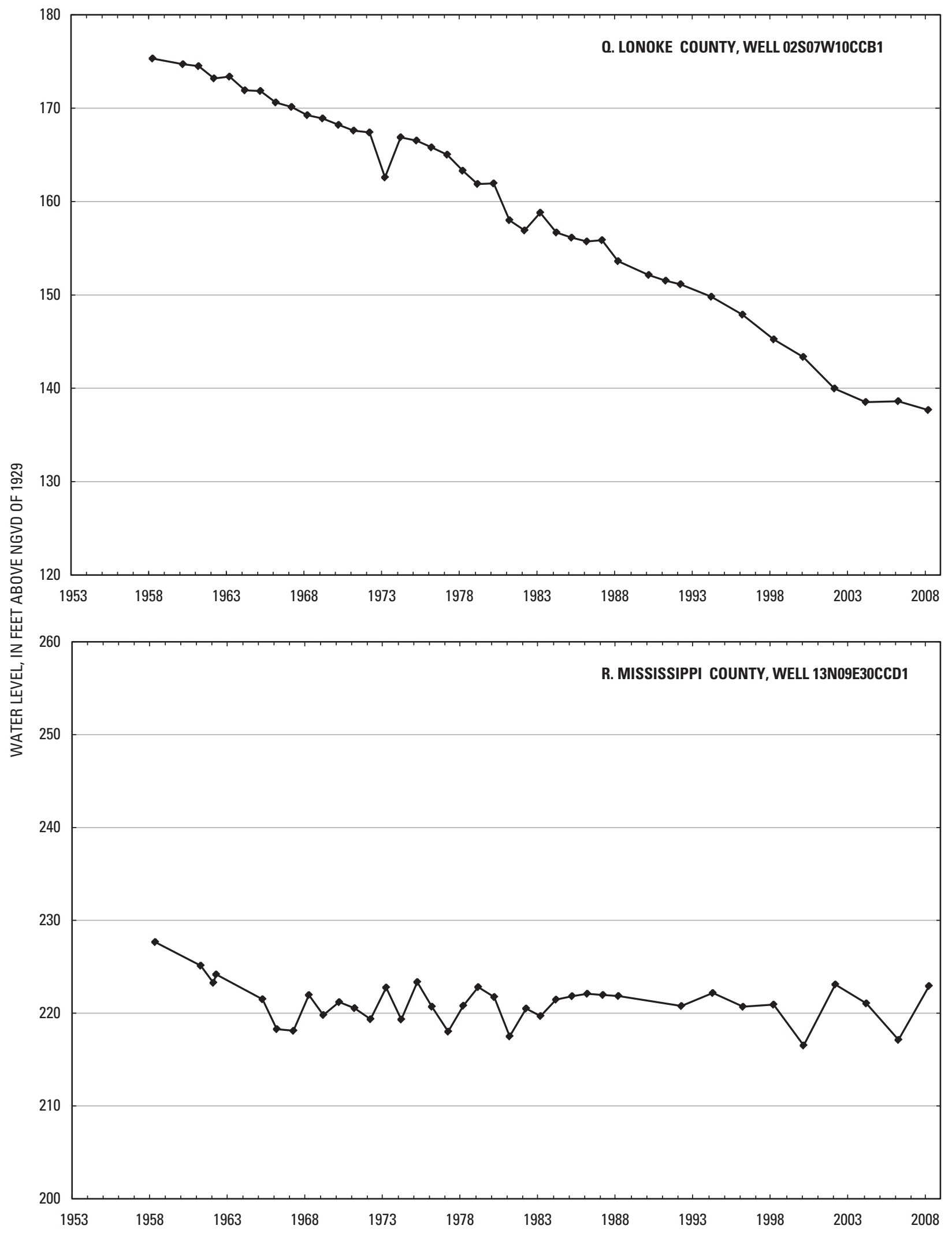

Figure 4. Water-level hydrographs (A to CC) for selected wells in the Mississippi River Valley alluvial aquifer-CContinued 


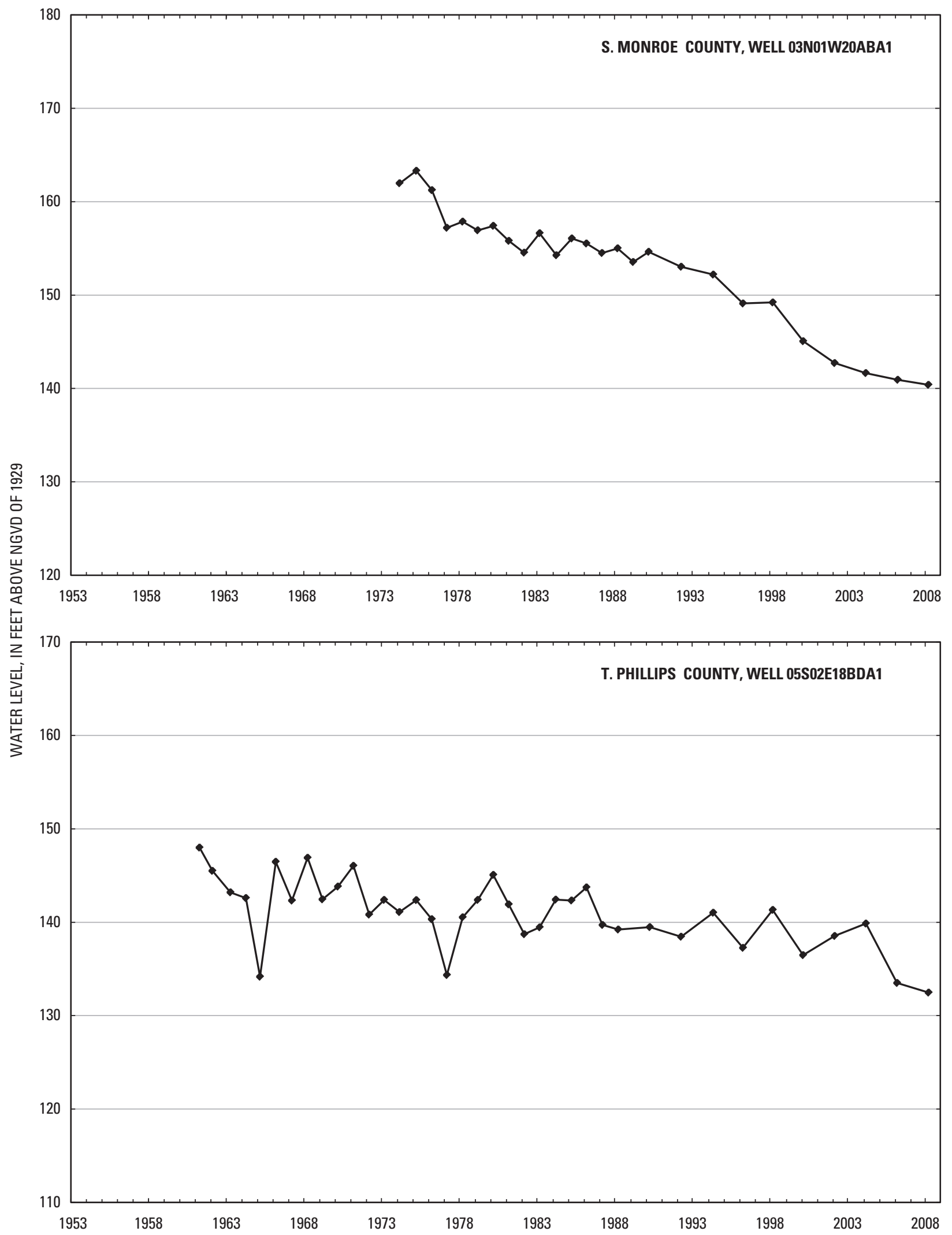

Figure 4. Water-level hydrographs (A to CC) for selected wells in the Mississippi River Valley alluvial aquifer.—Continued 


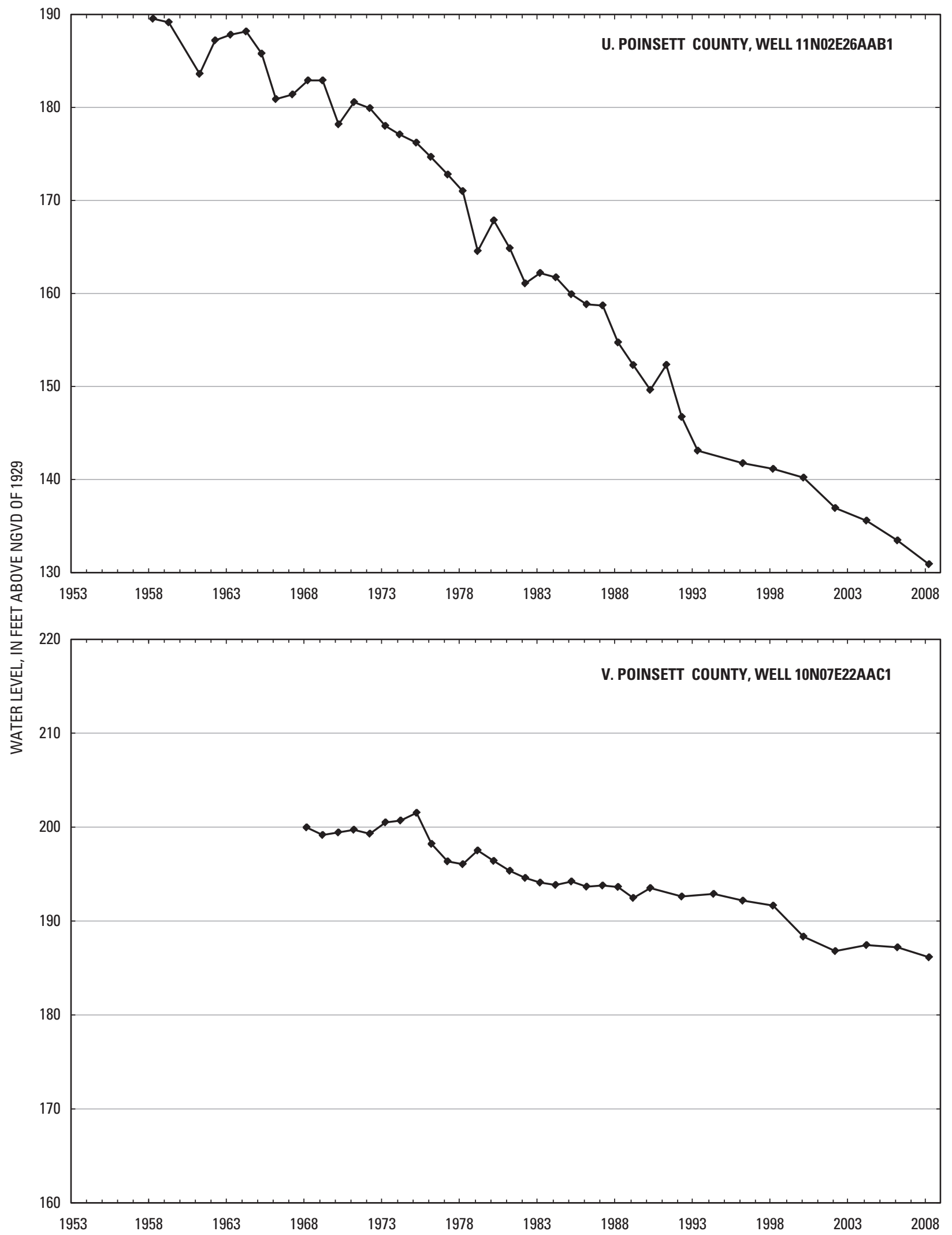

Figure 4. Water-level hydrographs (A to CC) for selected wells in the Mississippi River Valley alluvial aquifer-Continued 


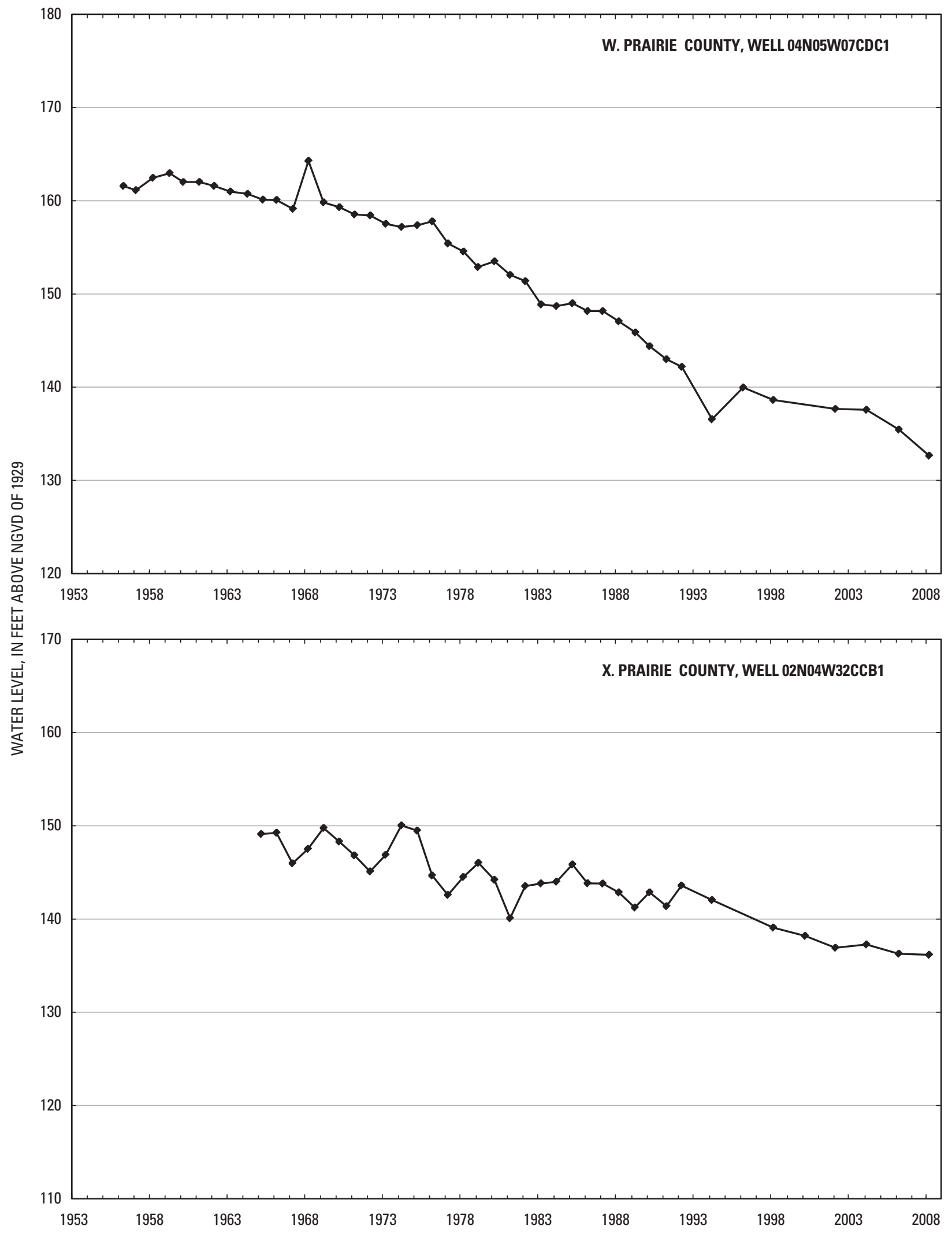

Figure 4. Water-level hydrographs (A to CC) for selected wells in the Mississippi River Valley alluvial aquifer-—Continued 


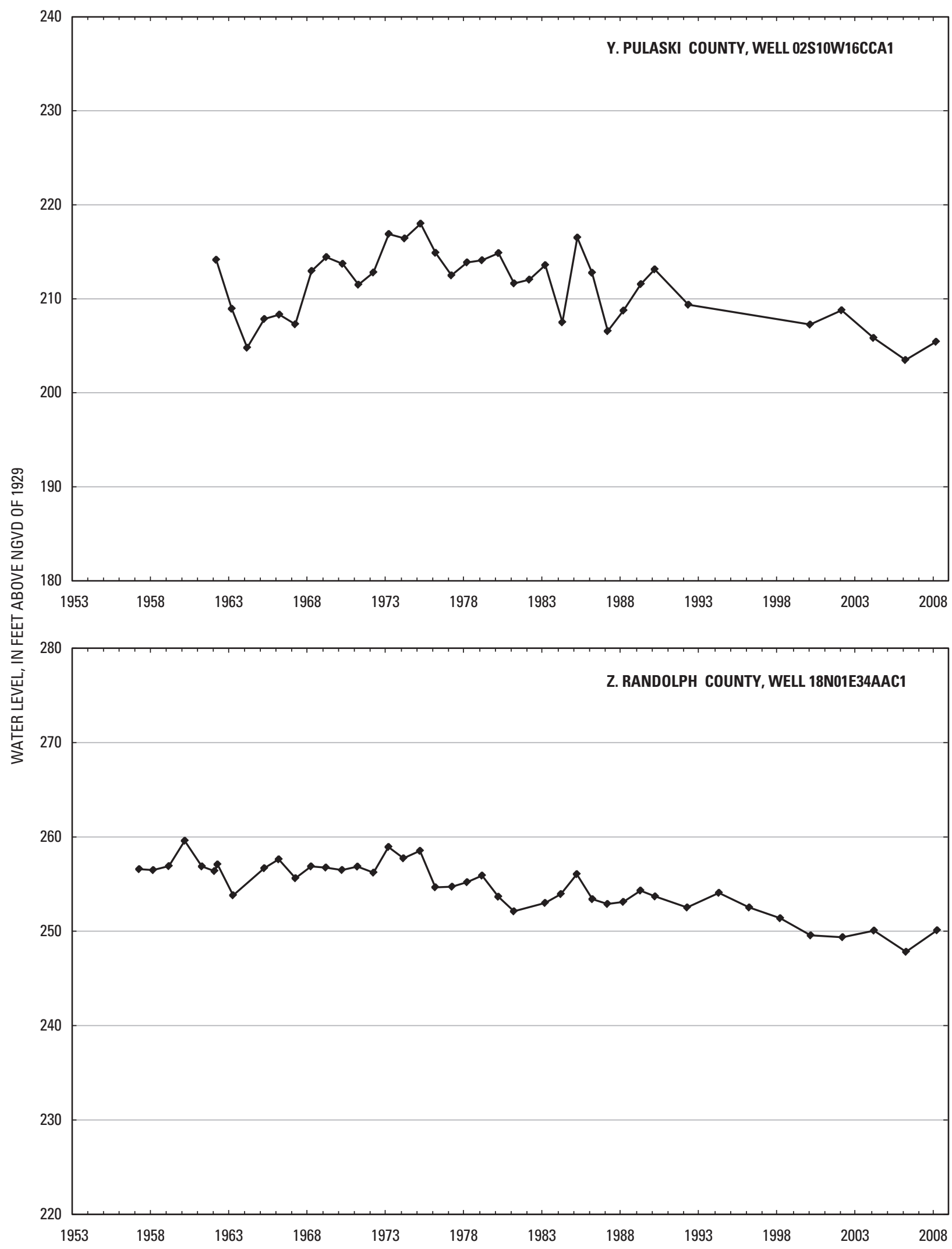

Figure 4. Water-level hydrographs (A to CC) for selected wells in the Mississippi River Valley alluvial aquifer-Continued 


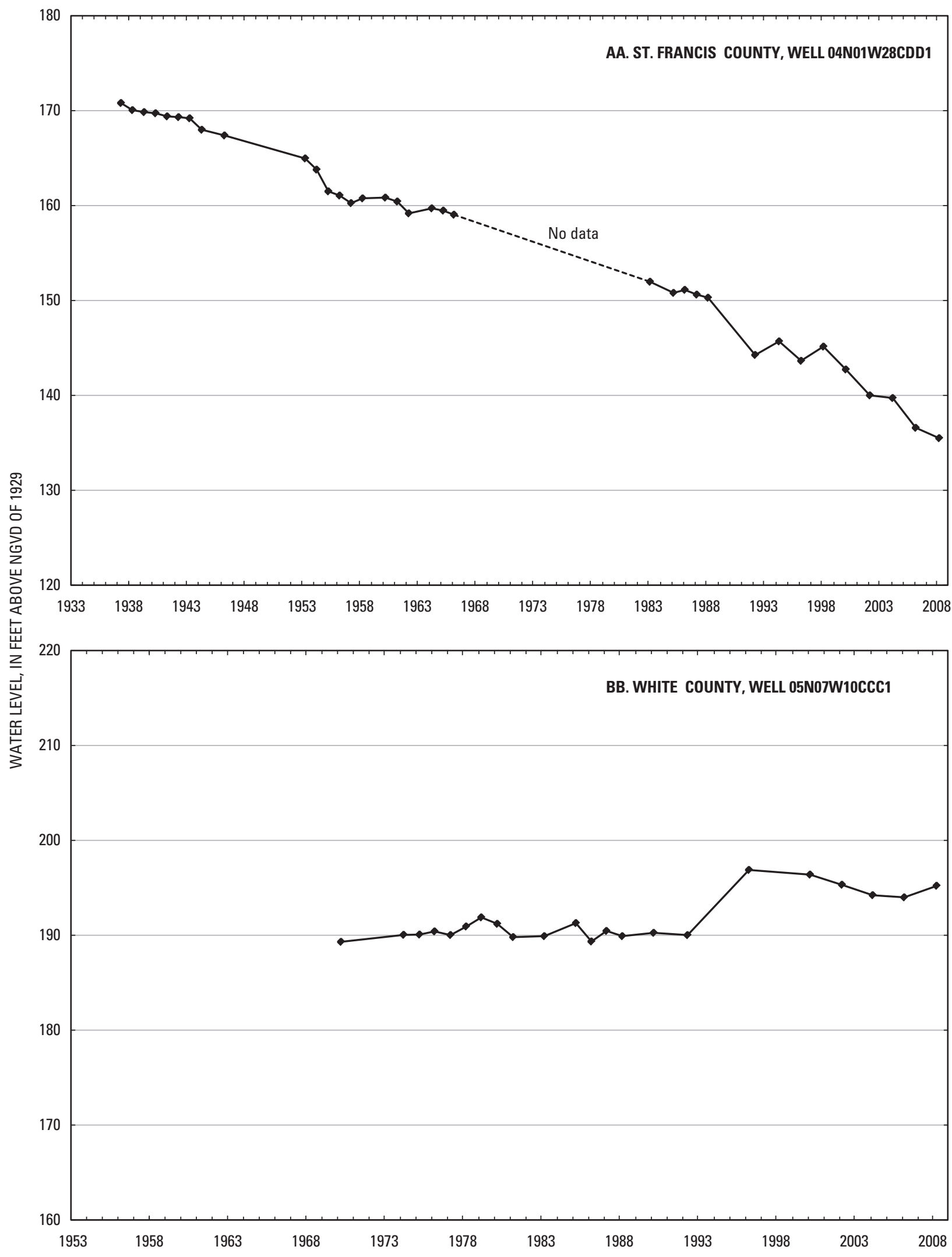

Figure 4. Water-level hydrographs (A to CC) for selected wells in the Mississippi River Valley alluvial aquifer-—Continued 


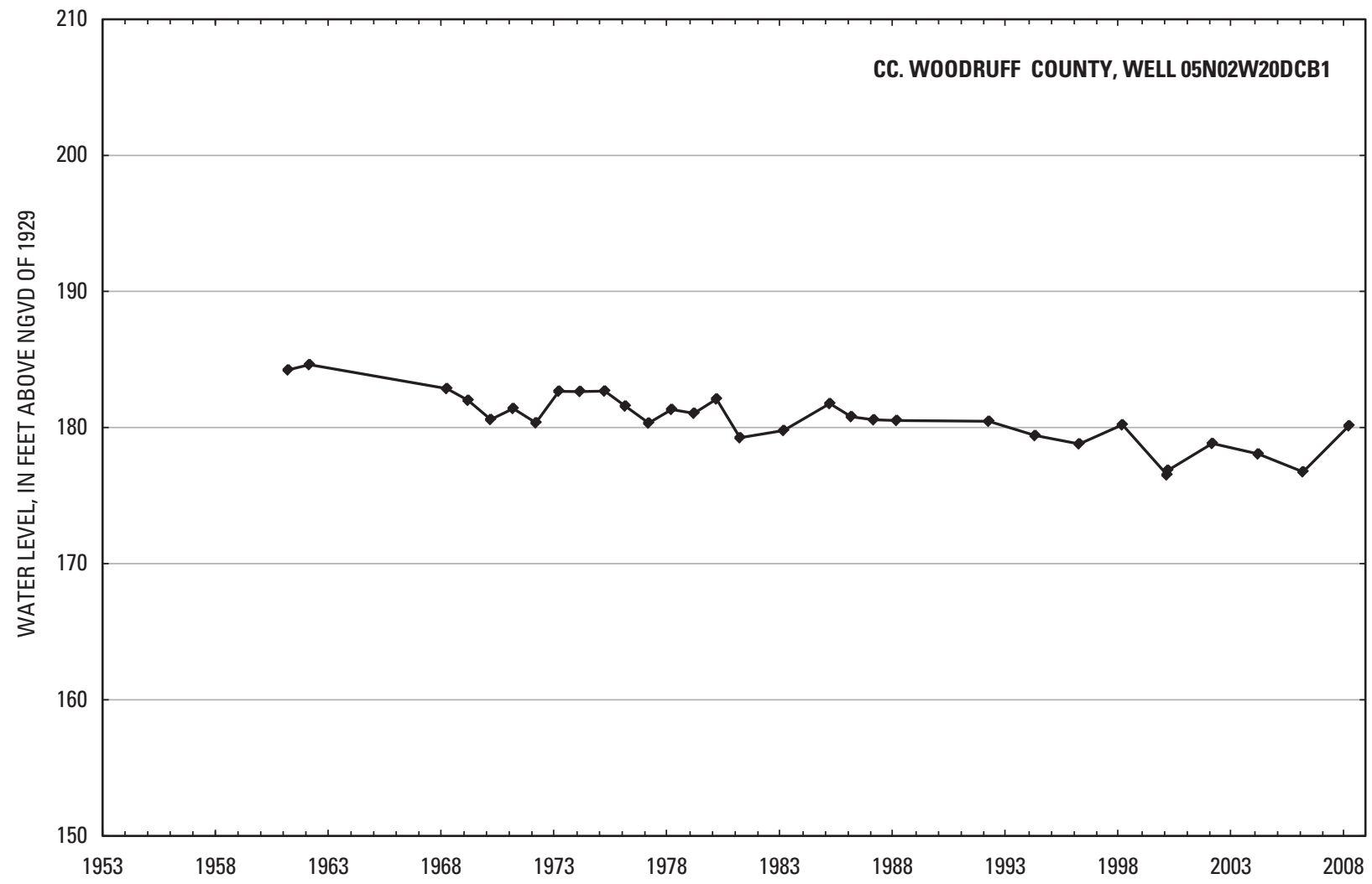

Figure 4. Water-level hydrographs (A to CC) for selected wells in the Mississippi River Valley alluvial aquifer-_Continued

\section{Water-Quality Conditions}

Water samples were collected from 60 wells completed in the alluvial aquifer and measured onsite for specific conductance and temperature (appendix 3). Specific conductance ranged from 111 microsiemens per centimeter at 25 degrees Celsius $(\mu \mathrm{S} / \mathrm{cm})$ at a well in Lincoln County to $2,020 \mu \mathrm{S} / \mathrm{cm}$ at a well in Desha County (appendix 3). The majority of the values are in the 401-600, 601-800, and 801-1,000 $\mu \mathrm{S} / \mathrm{cm}$ ranges (fig. 5). Specific conductance values equaled or exceeded $1,000 \mu \mathrm{S} / \mathrm{cm}$ in Arkansas, Chicot, Cross, Desha, Greene, and Lincoln Counties. Other values in Chicot County are as low as $393 \mu \mathrm{S} / \mathrm{cm}$, in Desha County as low as $725 \mu \mathrm{S} / \mathrm{cm}$, and in Lincoln County as low as $111 \mu \mathrm{S} / \mathrm{cm}$. Chicot, Desha and Lincoln Counties have a wide range of specific conductance values.

Generally, the occurrences of higher specific conductance in the alluvial aquifer are associated with the movement of water containing high concentrations of dissolved solids from sources at depth (Bryant and others, 1985). Water with higher concentrations of dissolved solids may have moved upward where the confining units are thin or absent, along faults, or through unplugged or deteriorated casings of abandoned oil and gas test wells (Fitzpatrick, 1985). Morris and Bush (1986) cite two possible sources of high dissolved-solids concentrations - a zone of groundwater stagnation present in the alluvial aquifer caused by localized restricted horizontal or vertical flow, and upward movement of water with higher dissolvedsolids concentration from deeper formations in response to pumping. The variability in specific conductance in Desha and Lincoln Counties is explained by the local geomorphology. Channel deposits have high recharge and permeability, resulting in lower specific conductance. Backswamp deposits have low recharge and permeability and higher evapotranspiration, resulting in higher specific conductance. The high specific conductance in Chicot County is explained by the upward migration of higher specific conductance water through the intersection of two faults (Kresse and Clark, 2008). The high specific conductance water is limited to a specific area, with lower specific conductance water in the rest of the county.

The histograms of specific conductance data for 2008 and 2006 are similarly shaped (figs. 5 and 6, respectively). The largest category for both years is the $401-600 \mu \mathrm{S} / \mathrm{cm}$ range. The number of values decreases by 5 for the $601-800 \mu \mathrm{S} / \mathrm{cm}$ range in 2008. Both histograms have a right skew. The number of samples collected in 2006 is 65,5 more than in 2008. Six wells sampled in 2008, 10 percent, were from the wells sampled in 2006. The mean specific conductance for both years was $393 \mu \mathrm{S} / \mathrm{cm}$. The similarity in distribution shape, largest category, and mean from two different well sets indicate a minimal change in water quality from 2006 to 2008. 


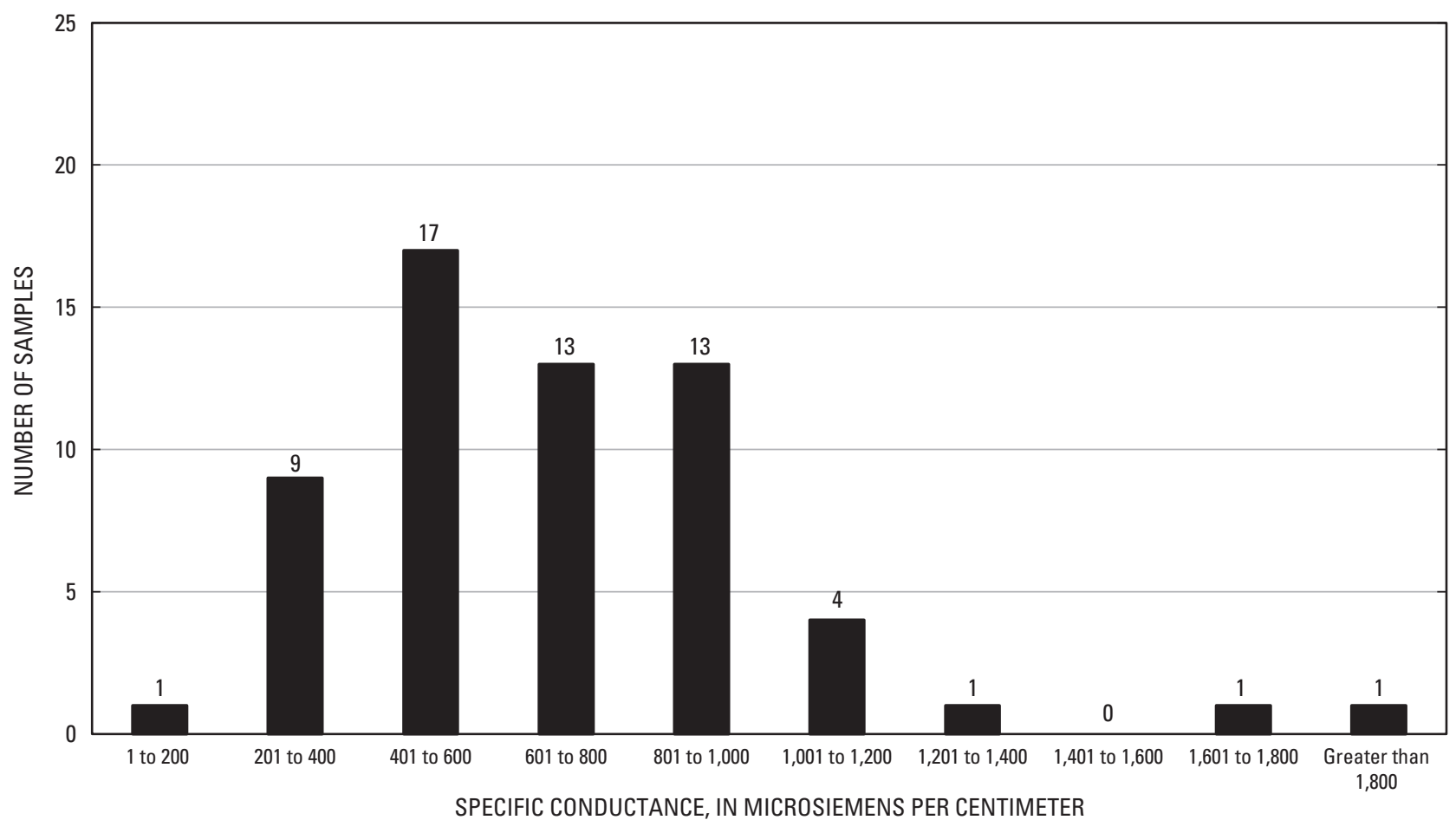

Figure 5. Distribution of specific conductance in samples from the Mississippi River Valley alluvial aquifer in 2008.

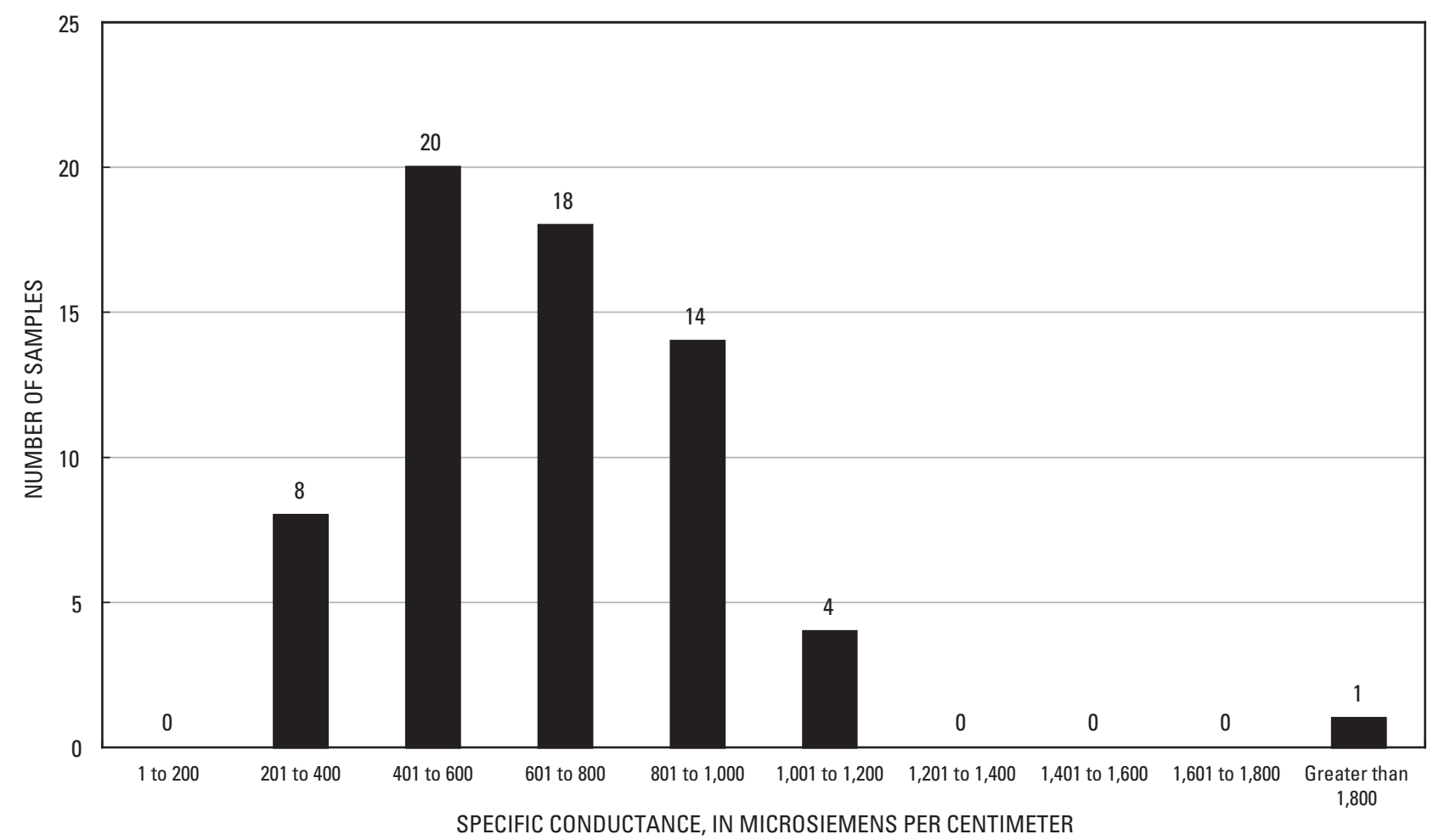

Figure 6. Distribution of specific conductance in samples from the Mississippi River Valley alluvial aquifer in 2006. 


\section{Summary}

The Mississippi River Valley alluvial aquifer is increasingly relied upon for agriculture and aquaculture in eastern Arkansas. Since 1965 withdrawals from the alluvial aquifer have increased from about 1,063 million gallons per day to about 7,252 million gallons per day in 2005, an increase of about 582 percent. Withdrawals have more than doubled in the last 20 years, about a 105 percent increase since 1985 .

Groundwater levels are affected by groundwater withdrawals within the study area, often resulting in depressions. In 2008, the lowest water-level altitude was $69 \mathrm{ft}$ in the center of Arkansas County. The highest water-level altitude was 288 $\mathrm{ft}$ in northeastern Clay County on the western side of Crowleys Ridge. Two large depressions in the potentiometric surface are located in Arkansas, Lonoke, and Prairie Counties and west of Crowleys Ridge in Craighead, Cross, Lee, Monroe, Poinsett, St. Francis, and Woodruff Counties. The elongated depression in Arkansas, Lonoke, and Prairie Counties has two areas that have changed in horizontal area or depth when compared to previous conditions of the aquifer. The area in Arkansas County within the southeastern half of the depression has not expanded horizontally during recent years, although the center of the depression has deepened. The Arkansas and White Rivers that bound Arkansas County on the southwestern and eastern county lines are hydrologically connected and provide recharge to the alluvial aquifer. The area in Lonoke and Prairie Counties in the northwestern half of the depression has not expanded, and water level in the deeper part of the depression has risen. The 90-foot contour shown in the 2006 potentiometric surface is not shown on the 2008 potentiometric-surface map. Along the western side of Crowleys Ridge, the area enclosed by a 140-foot contour in Cross and Poinsett Counties has expanded further south into Cross County. The 130 -foot contour in St. Francis, Monroe, and Woodruff Counties in 2006 is not shown in the 2008 potentiometric-surface map. A 130-foot contour in Poinsett County has expanded north in 2008. A 130-foot contour is shown in Cross County that was not evident in previous years.

Three other areas of reduced water level were identified in previous work in southeastern Arkansas - one in eastern Lincoln County, a second that extends from southern Desha County into northern Chicot County, and a third that extends from western Chicot County into eastern Ashley County. The area with reduced water levels in western Chicot and eastern Ashley Counties is about the same size in 2008 and 2006. The water levels in this area do not have a trend, where some water levels have risen and some have declined 1 to $2 \mathrm{ft}$ since 2006. From 2004 to 2008, in western Chicot and eastern Ashley Counties the depth of this area has not increased and is approximately the same area. The area in eastern Lincoln County expanded into northwestern Desha County and continued to deepen through 2004 with an altitude of $118 \mathrm{ft}$ at its deepest point. In 2006, this area expanded westward in Lincoln County.
Six depressions are shown in the 2008 potentiometricsurface map, three that were first shown in the 2006 potentiometric surface and two that have not been previously shown. A depression at the Prairie and White County line, a second depression at the Craighead and Mississippi County line, and a small depression in northeastern St. Francis County were first shown on the 2006 potentiometric-surface map. A depression in central St. Francis County on the western side of Crowleys Ridge was first shown on the 2002 potentiometric-surface map. A depression in eastern Randolph County and a depression in north-central Ashley County have not been shown on previous potentiometric-surface maps. A depression in Jefferson County was shown on the 2006 potentiometric-surface map but is not shown on the 2008 potentiometric-surface map.

The regional direction of groundwater flow is generally to the south and east except where flow is affected by groundwater withdrawals; however, the flow direction is affected over substantial areas by depressions. West of Crowleys Ridge, depressions in Arkansas, Lonoke, and Prairie Counties capture ground-water flow from all directions. The flow along large sections of the Arkansas, Mississippi, and White Rivers is away from the rivers. East of Crowleys Ridge water flows from north to south along Crowleys Ridge and northeast to southwest along the Mississippi River. South of the Arkansas River the flow is towards the southeast, except in northwestern Desha County where flow is towards the area of reduced water level.

A map showing the difference in water level was constructed using 595 differences in water levels measured in 585 wells during 2008 and 2004. The difference in measured water levels from 2004 to 2008 ranged from $-20.6 \mathrm{ft}$ to $25.9 \mathrm{ft}$, with a mean of $-1.6 \mathrm{ft}$. The largest decline of $-20.6 \mathrm{ft}$ occurred in Randolph County and the largest rise of $25.9 \mathrm{ft}$ occurred in Prairie County. Out of the 595 differences, 442 were declines (74.3 percent), 10 were no difference (values of $0.0 \mathrm{ft})(1.7$ percent), and 143 were rises (24.0 percent). Five areas are dominated by declines that are west of Crowleys Ridge; in eastern Craighead County; in southern Mississippi and Crittenden Counties; in eastern Lonoke and western Prairie Counties; and in Arkansas, Ashley, Chicot, Desha, Drew, and Lincoln Counties. Six areas are dominated by rises in measured water levels; in northern Prairie, White, and western Woodruff Counties adjacent to the White River, in Jefferson County, in northern Mississippi County, in western Greene and Randolph Counties, Independence and northwestern Jackson Counties, and eastern Greene County.

Long-term water-level changes were evaluated using hydrographs from 173 wells in the alluvial aquifer for the period 1984 to 2008 . The mean annual rise or decline in water level for the entire study area was -0.38 feet per year $(\mathrm{ft} / \mathrm{yr})$ with a range of -4.86 to $0.58 \mathrm{ft} / \mathrm{yr}$. Long-term water-level changes vary substantially across the study area. Independence and White Counties are the only counties with a mean annual rise from 1984 to 2008. The rise in Independence County is determined from the data of one well. Mean annual declines between $-0.50 \mathrm{ft} / \mathrm{yr}$ and $0.00 \mathrm{ft} / \mathrm{yr}$ occurred in Arkansas, 
Chicot, Clay, Craighead, Crittenden, Drew, Greene, Jefferson, Mississippi, Monroe, Phillips, Poinsett, Prairie, Pulaski, Randolph, and Woodruff Counties. Mean annual declines between $-1.00 \mathrm{ft} / \mathrm{yr}$ and $-0.50 \mathrm{ft} / \mathrm{yr}$ occurred in Ashley, Desha, Jackson, Lee, Lincoln, and St. Francis Counties. Mean annual declines between $-1.50 \mathrm{ft} / \mathrm{yr}$ and $-1.00 \mathrm{ft} / \mathrm{yr}$ occurred in Cross and Lonoke Counties.

The analysis of long-term water-level changes in Arkansas, Lonoke, and Prairie Counties shows the elongation of the depression in these three counties. Arkansas and Prairie Counties have two different rates of annual decline for the two hydrographs shown for each county. Water levels in the two wells near the Arkansas and White Rivers either have risen or declined at a slower rate than in the three wells in the center, northern, and western part of the depression. These rates of water-level change indicate that this depression has expanded in an elongated direction north and west into Lonoke and Prairie Counties from 1984 to 2008. The depression west of Crowleys Ridge has six wells with hydrographs in or near the depression that can be used to characterize the rates of waterlevel change within the depression.

Water samples were collected from 60 wells completed in the alluvial aquifer and measured onsite for specific conductance and temperature. Specific conductance ranged from 111 microsiemens per centimeter at 25 degrees Celsius $(\mu \mathrm{S} / \mathrm{cm})$ at a well in Lincoln County to $2,020 \mu \mathrm{S} / \mathrm{cm}$ at a well in Desha County. Specific conductance values equaled or exceeded $1,000 \mu \mathrm{S} / \mathrm{cm}$ in Arkansas, Chicot, Cross, Desha, Greene, and Lincoln Counties.

\section{Selected References}

Ackerman, D.J., 1996, Hydrology of the Mississippi River Valley alluvial aquifer, south-central United States-A preliminary assessment of the regional flow system: U.S. Geological Survey Professional Paper 1416-D, 56 p.

Boswell, E.H., Cushing, E.M., and Hosman, R.L., 1968, Quaternary aquifers in the Mississippi Embayment with a discussion of Quality of the water by H.G. Jeffery: U.S. Geological Survey Professional Paper 448-E, 15 p.

Broom, M.E., and Reed, J.E., 1973, Hydrology of the Bayou Bartholomew alluvial aquifer-stream system, Arkansas: U.S. Geological Survey Open-File Report 73-34, 91 p.

Bryant, C.T., Ludwig, A.H., and Morris, E.E., 1985, Ground water problems in Arkansas: U.S. Geological Survey WaterResources Investigations Report 85-4010, 24 p.

Fenneman, N.M., 1938, Physiography of eastern United States: New York, McGraw-Hill Book Co. Inc., 689 p.

Freiwald, D.A., 1984, Average annual precipitation and runoff for Arkansas, 1951-80: U.S. Geological Survey WaterResources Investigations Report 84-4363, 1 sheet.
Fitzpatrick, D.J., 1985, Occurrence of saltwater in the alluvial aquifer in the Boeuf-Tensas Basin, Arkansas: U.S. Geological Survey Water-Resources Investigation Report 85-4029, 1 sheet.

Gonthier, G.J., and Mahon, G.L., 1993, Thickness of the Mississippi River Valley confining unit, eastern Arkansas: U.S. Geological Survey Water-Resources Investigation Report 92-4121, 4 sheets.

Halberg, H.N., 1972, Use of water in Arkansas, 1970: Arkansas Geological Commission Water Resources Summary Number 7, $17 \mathrm{p}$.

Halberg, H.N., 1977, Use of water in Arkansas, 1975: Arkansas Geological Commission Water Resources Summary Number 9, 28 p.

Halberg, H.N., and Stephens, J.W., 1966, Use of water in Arkansas, 1965: Arkansas Geological Commission Water Resources Summary Number 5, 12 p.

Helsel, D.R., and Hirsch, R.M., 1992, Statistical methods in water resources: New York, NY, Elsevier Science Publishing Co., $522 \mathrm{p}$.

Holland, T.W., 1987, Use of water in Arkansas, 1985: Arkansas Geological Commission Water Resources Summary Number 14, $30 \mathrm{p}$.

Holland, T.W., 1993, Use of water in Arkansas, 1990: U.S. Geological Survey Open-File Report 93-48, pamphlet.

Holland, T.W., 1999, Water use in Arkansas, 1995: U.S. Geological Survey Open-File Report 99-188, 1 sheet.

Holland, T.W., 2004, Estimated water use in Arkansas, 2000: U.S. Geological Survey Scientific Investigations Report 2004-5230, $31 \mathrm{p}$.

Holland, T.W., 2007, Water use in Arkansas, 2005: U.S. Geological Survey Scientific Investigations Report 2007-5241, $31 \mathrm{p}$.

Holland, T.W., and Ludwig, A.H., 1981, Use of water in Arkansas, 1980: Arkansas Geological Commission Water Resources Summary Number 14, 30 p.

Kresse, T.M., and Clark, B.R., 2008, Occurrence, distribution, sources, and trends of elevated chloride concentrations in the Mississippi River Valley alluvial aquifer in southeastern Arkansas: U.S. Geological Survey Scientific Investigations Report 2008-5193, 34 p.

Joseph, R.L., 1999, Status of water levels and selected waterquality conditions in the Mississippi River Valley alluvial aquifer in eastern Arkansas, 1998: U.S. Geological Survey Water-Resources Investigations Report 99-4035, 54 p. 
Mahon, G.L., and Poynter, D.T., 1993, Development, calibration, and testing of ground-water flow models for the Mississippi River Valley alluvial aquifer in eastern Arkansas using one-square mile cells: U.S. Geological Survey WaterResources Investigations Report 92-4106, 33 p.

Morris, E.E., and Bush, W.V., 1986, Extent and source of saltwater intrusion into the alluvial aquifer near Brinkley, Arkansas, 1984: U.S. Geological Survey Water-Resources Investigations Report 85-4322, 123 p.

Pugh, A.L., Westerfield, P.W., and Poynter, D.T., 1997, Thickness of the Mississippi River Valley alluvial aquifer in eastern Arkansas: U.S. Geological Survey Water-Resources Investigations Report 97-4049, 1 sheet.

Reed, T.B., 2004, Status of water levels and selected waterquality conditions in the Mississippi River Valley alluvial aquifer in eastern Arkansas, 2002: U.S. Geological Survey Scientific Investigations Report 2004-5129, 53 p.

Schrader, T.P., 2001, Status of water levels and selected waterquality conditions in the Mississippi River Valley alluvial aquifer in eastern Arkansas, 2000: U.S. Geological Survey Water-Resources Investigations Report 01-4124, 52 p.

Schrader, T.P., 2006, Status of water levels and selected waterquality conditions in the Mississippi River Valley alluvial aquifer in eastern Arkansas, 2004: U.S. Geological Survey Scientific Investigations Report 2006-5128, 82 p.

Schrader, T.P., 2008, Water levels and selected water-quality conditions in the Mississippi River Valley alluvial aquifer in eastern Arkansas, 2006: U.S. Geological Survey Scientific Investigations Report 2008-5092, 72 p.

Stanton, G.P., Joseph, R.L., and Pugh, A.L., 1998, Status of water levels and selected water-quality conditions in the Mississippi River Valley alluvial aquifer in eastern Arkansas, 1994-1996: U.S. Geological Survey Water-Resources Investigations Report 98-4131, 72 p. 

Appendixes 1-3 
Appendix 1. Information pertaining to water levels measured in wells completed in the Mississippi River Valley alluvial aquifer in eastern Arkansas, spring 2008.

[USGS, U.S. Geological Survey; NRCS, Natural Resources Conservation Service, ANRC, Arkansas Natural Resources Commission; --, no data; NGVD of 1929, National Geodetic Vertical Datum of 1929; Horizontal coordinate information is referenced to the North American Datum of 1983 (NAD 83 ); *, control wells for duplicate measurements by USGS and NRCS for quality assurance]

\begin{tabular}{|c|c|c|c|c|c|c|c|c|}
\hline Station nme & $\begin{array}{c}\text { Latitude } \\
\text { (degrees, } \\
\text { minutes, } \\
\text { seconds) }\end{array}$ & $\begin{array}{l}\text { Longitude } \\
\text { (degrees, } \\
\text { minutes, } \\
\text { seconds) }\end{array}$ & $\begin{array}{l}\text { Source } \\
\text { of data }\end{array}$ & $\begin{array}{c}\text { Depth of } \\
\text { well } \\
\text { (feet) }\end{array}$ & $\begin{array}{l}\text { Land-surface } \\
\text { datum altitude } \\
\text { (feet above } \\
\text { NGVD of } \\
\text { 1929) }\end{array}$ & $\begin{array}{c}\text { Depth to } \\
\text { water } \\
\text { (feet below } \\
\text { land-surface } \\
\text { datum) }\end{array}$ & $\begin{array}{c}\text { Water-leve } \\
\text { altitude } \\
\text { (feet above } \\
\text { NGVD of } \\
\text { 1929) }\end{array}$ & $\begin{array}{c}\text { Date of } \\
\text { measure- } \\
\text { ment }\end{array}$ \\
\hline \multicolumn{9}{|c|}{ Arkansas County } \\
\hline 02S04W11DBB1 & 343233 & 912415 & USGS & 152 & 213.04 & 100.74 & 112 & $03 / 24 / 2008$ \\
\hline 02S05W15AAB1 & 343213 & 913127 & USGS & 180 & 213 & 107.38 & 106 & 03/24/2008 \\
\hline 02S05W31BBB1 & 342937 & 913536 & USGS & 90 & 198 & 41.24 & 157 & 03/24/2008 \\
\hline 03S02W27ABB1 & 342448 & 911251 & USGS & 87 & 197 & 65.92 & 131 & $03 / 25 / 2008$ \\
\hline 03S03W05CCD1 & 342737 & 912132 & ANRC & 150 & 201 & 99.78 & 101 & 03/24/2008 \\
\hline 03S03W27BBC1 & 342455 & 911944 & ANRC & 120 & 195 & 93.04 & 102 & 03/24/2008 \\
\hline 03S04W02BBB1 & 342831 & 912454 & USGS & 116 & 197.63 & 93.22 & 104 & 03/24/2008 \\
\hline 03S04W03DCA16 & 342753 & 912515 & USGS & 126 & 205 & 101.14 & 104 & 05/12/2008 \\
\hline 03S04W03DCA6 & 342753 & 912517 & USGS & 122.3 & 204 & 100.63 & 103 & 03/24/2008 \\
\hline 03S04W03DDA1 & 342750 & 912460 & USGS & 127 & 202 & 100.94 & 101 & 03/24/2008 \\
\hline 03S05W03CCC1 & 342752 & 913227 & USGS & 110 & 215 & 104.42 & 111 & 03/20/2008 \\
\hline 03S06W35ADD1 & 342411 & 913652 & USGS & -- & 190 & 54.81 & 135 & 03/21/2008 \\
\hline 04S01W04ACD2 & 342233 & 910733 & USGS & 52.4 & 155 & 4.05 & 151 & 03/25/2008 \\
\hline 04S01W31DCB1 & 341753 & 910949 & ANRC & 130 & 179 & 52.42 & 127 & 03/25/2008 \\
\hline 04S02W11AAA1 & 342209 & 911123 & USGS & -- & 195.08 & 67.81 & 127 & $03 / 25 / 2008$ \\
\hline 04S02W29CCC1 & 341846 & 911539 & USGS & 140 & 191 & 83.92 & 107 & 03/25/2008 \\
\hline 04S03W17ADD1 & 342102 & 912058 & USGS & -- & 200 & 109.98 & 90 & 03/24/2008 \\
\hline 04S03W32BCB1 & 341820 & 912202 & USGS & -- & 192 & 123.19 & 69 & 03/20/2008 \\
\hline 04S04W02ABB1 & 342313 & 912424 & USGS & 155 & 200 & 109.89 & 90 & 03/24/2008 \\
\hline 04S04W35ABC1 & 341835 & 912437 & NRCS & -- & 193 & 106 & 87 & 04/07/2008 \\
\hline 04S05W16CDC1 & 342045 & 913321 & USGS & 120 & 201 & 71.25 & 130 & 03/20/2008 \\
\hline 04S05W24DAA1 & 342001 & 912930 & USGS & 150 & 198 & 90.17 & 108 & 03/20/2008 \\
\hline 04S06W15DBB1 & 342122 & 913827 & USGS & 100 & 190 & 34.88 & 155 & 03/21/2008 \\
\hline 05S01W16BAB1 & 341552 & 910729 & USGS & -- & 183 & 51.37 & 132 & 03/25/2008 \\
\hline 05S02W16ABD1 & 341552 & 911358 & USGS & 154 & 190 & 86.13 & 104 & 03/20/2008 \\
\hline 05S04W07CCC1 & 341555 & 912932 & USGS & 120 & 194 & 75.05 & 119 & 03/20/2008 \\
\hline 05S04W32BBA1 & 341316 & 912822 & USGS & -- & 191 & 58.75 & 132 & 03/20/2008 \\
\hline 05S06W02DDD1 & 341724 & 913651 & USGS & 60 & 182.93 & 21.8 & 161 & 03/21/2008 \\
\hline 05S06W07DDC1 & 341642 & 914130 & USGS & 32 & 180.48 & 3.83 & 177 & 03/21/2008 \\
\hline 06S02W23DCD1 & 340853 & 911206 & USGS & -- & 188 & 70.61 & 117 & 03/20/2008 \\
\hline 06S03W10BBA1 & 341136 & 911954 & USGS & 155 & 184 & 82.25 & 102 & 03/20/2008 \\
\hline 06S03W27AAA1 & 340858 & 911913 & USGS & 132 & 183.14 & 68.55 & 115 & 03/20/2008 \\
\hline 07S02W04BBB1 & 340707 & 911452 & USGS & -- & 176 & 50.5 & 126 & 03/20/2008 \\
\hline 07S02W17BBA1 & 340530 & 911539 & USGS & 95 & 184 & 54.05 & 130 & 03/20/2008 \\
\hline 07S03W18CCD1 & 340435 & 912316 & USGS & -- & 186.18 & 44.17 & 142 & 03/20/2008 \\
\hline
\end{tabular}


Appendix 1. Information pertaining to water levels measured in wells completed in the Mississippi River Valley alluvial aquifer in eastern Arkansas, spring 2008._-Continued

[USGS, U.S. Geological Survey; NRCS, Natural Resources Conservation Service, ANRC, Arkansas Natural Resources Commission; --, no data; NGVD of 1929, National Geodetic Vertical Datum of 1929; Horizontal coordinate information is referenced to the North American Datum of 1983 (NAD 83 ); *, control wells for duplicate measurements by USGS and NRCS for quality assurance]

\begin{tabular}{|c|c|c|c|c|c|c|c|c|}
\hline Station nme & $\begin{array}{c}\text { Latitude } \\
\text { (degrees, } \\
\text { minutes, } \\
\text { seconds) }\end{array}$ & $\begin{array}{c}\text { Longitude } \\
\text { (degrees, } \\
\text { minutes, } \\
\text { seconds) }\end{array}$ & $\begin{array}{l}\text { Source } \\
\text { of data }\end{array}$ & $\begin{array}{c}\text { Depth of } \\
\text { well } \\
\text { (feet) }\end{array}$ & $\begin{array}{l}\text { Land-surface } \\
\text { datum altitude } \\
\text { (feet above } \\
\text { NGVD of } \\
\text { 1929) }\end{array}$ & $\begin{array}{c}\text { Depth to } \\
\text { water } \\
\text { (feet below } \\
\text { land-surface } \\
\text { datum) }\end{array}$ & $\begin{array}{c}\text { Water-leve } \\
\text { altitude } \\
\text { (feet above } \\
\text { NGVD of } \\
\text { 1929) }\end{array}$ & $\begin{array}{c}\text { Date of } \\
\text { measure- } \\
\text { ment }\end{array}$ \\
\hline \multicolumn{9}{|c|}{ Arkansas County-Continued } \\
\hline 07S03W32BBC1 & 340240 & 912216 & USGS & 128 & 176.92 & 26.45 & 150 & 03/20/2008 \\
\hline 07S04W01DDD1 & 340625 & 912327 & USGS & 155 & 186 & 48.48 & 138 & 03/20/2008 \\
\hline 08S02W08ACA1 & 340041 & 911506 & USGS & -- & 179 & 42.67 & 136 & 03/20/2008 \\
\hline 08S03WT2299 & 340147 & 912203 & USGS & 158 & 178 & 22.09 & 156 & 03/20/2008 \\
\hline \multicolumn{9}{|c|}{ Ashley County } \\
\hline 15S04W23DBD1 & 332247 & 912852 & USGS & -- & 128 & 33.42 & 95 & 03/11/2008 \\
\hline 15S04W26DCC1 & 332232 & 912902 & USGS & 64.1 & 127 & 32.17 & 95 & 03/11/2008 \\
\hline 15S07W21CBA1 & 332316 & 915001 & USGS & 27.4 & 210 & 4.52 & 205 & 03/10/2008 \\
\hline 16S06W08CAA1 & 331941 & 914438 & USGS & 105 & 185 & 78.92 & 106 & 03/10/2008 \\
\hline 16S06W25DDD1 & 331640 & 913958 & ANRC & 130 & 182 & 78.47 & 104 & 03/12/2008 \\
\hline 16S06W27BAB1 & 331729 & 914240 & USGS & 115 & 182 & 84.07 & 98 & 03/10/2008 \\
\hline 17S04W03ABB1 & 331528 & 913010 & USGS & 105 & 124 & 30.77 & 93 & 03/11/2008 \\
\hline 17S04W15DDC1 & 331252 & 912954 & USGS & 57 & 116 & 27.70 & 88 & 03/11/2008 \\
\hline 17S04W21ABA1 & 331252 & 913108 & USGS & -- & 117 & 22.97 & 94 & 03/11/2008 \\
\hline 17S05W01AAD1 & 331459 & 913402 & NRCS & 100 & 122 & 18 & 104 & 04/15/2008 \\
\hline 17S06W01ADD1 & 331518 & 913956 & USGS & 144 & 182 & 84.03 & 98 & 03/10/2008 \\
\hline 17S06W35CAC1 & 331049 & 914136 & USGS & 140 & 179 & 72.41 & 107 & 03/10/2008 \\
\hline 18S04W23DDD1 & 330658 & 912856 & NRCS & 100 & 103 & 30 & 73 & $04 / 15 / 2008$ \\
\hline 18S05W11CCD1 & 330841 & 913538 & NRCS & 75 & 118 & 27 & 91 & 04/15/2008 \\
\hline 18S05W22DDA1 & 330712 & 913555 & NRCS & 100 & 125 & 22 & 103 & 04/15/2008 \\
\hline 18S08W01AAB1 & 331015 & 915225 & USGS & 128 & 181 & 84.34 & 97 & 03/10/2008 \\
\hline 18S08W28DDD2 & 330625 & 915528 & USGS & 156 & 163.26 & 85.11 & 78 & 05/13/2008 \\
\hline 19S04W06BAB2 & 330504 & 913329 & USGS & 98 & 110 & 23.92 & 86 & 03/11/2008 \\
\hline 19S04W14BBB1 & 330310 & 912913 & NRCS & 100 & 107 & 31 & 76 & 04/15/2008 \\
\hline 19S05W08ACA1 & 330405 & 913815 & NRCS & -- & 111 & 18 & 93 & 04/15/2008 \\
\hline 19S05W16ABB1 & 330323 & 913718 & NRCS & 100 & 116 & 28 & 88 & $04 / 15 / 2008$ \\
\hline 19S05W22DCD1 & 330139 & 913615 & NRCS & -- & 107 & 26 & 81 & 04/15/2008 \\
\hline 19S06W07BCC1 & 330404 & 914608 & USGS & -- & 134.7 & 32.46 & 102 & 03/10/2008 \\
\hline \multicolumn{9}{|c|}{ Chicot County } \\
\hline 13S03W27AAA1 & 333253 & 912310 & NRCS & -- & 138 & 48 & 90 & $03 / 24 / 2008$ \\
\hline 13S03W34BAA1 & 333110 & 912539 & USGS & 100 & 133 & 40.74 & 92 & 03/11/2008 \\
\hline 13S03W34CAA1 & 333136 & 912336 & USGS & 75 & 132 & 37.92 & 94 & 03/11/2008 \\
\hline 13S03W35BAC1 & 333154 & 912246 & USGS & 90 & 134 & 41.29 & 93 & 03/11/2008 \\
\hline 14S02W09BDD1 & 332859 & 911729 & NRCS & -- & 133 & 30 & 103 & 03/26/2008 \\
\hline 14S02W18BBDD1 & 332859 & 912038 & NRCS & -- & 129 & 34 & 95 & 03/24/2008 \\
\hline
\end{tabular}


Appendix 1. Information pertaining to water levels measured in wells completed in the Mississippi River Valley alluvial aquifer in eastern Arkansas, spring 2008._-Continued

[USGS, U.S. Geological Survey; NRCS, Natural Resources Conservation Service, ANRC, Arkansas Natural Resources Commission; --, no data; NGVD of 1929, National Geodetic Vertical Datum of 1929; Horizontal coordinate information is referenced to the North American Datum of 1983 (NAD 83 ); *, control wells for duplicate measurements by USGS and NRCS for quality assurance]

\begin{tabular}{|c|c|c|c|c|c|c|c|c|}
\hline Station nme & $\begin{array}{l}\text { Latitude } \\
\text { (degrees, } \\
\text { minutes, } \\
\text { seconds) }\end{array}$ & $\begin{array}{c}\text { Longitude } \\
\text { (degrees, } \\
\text { minutes, } \\
\text { seconds) }\end{array}$ & $\begin{array}{l}\text { Source } \\
\text { of data }\end{array}$ & $\begin{array}{c}\text { Depth of } \\
\text { well } \\
\text { (feet) }\end{array}$ & $\begin{array}{l}\text { Land-surface } \\
\text { datum altitude } \\
\text { (feet above } \\
\text { NGVD of } \\
\text { 1929) }\end{array}$ & $\begin{array}{c}\text { Depth to } \\
\text { water } \\
\text { (feet below } \\
\text { land-surface } \\
\text { datum) }\end{array}$ & $\begin{array}{l}\text { Water-level } \\
\text { altitude } \\
\text { (feet above } \\
\text { NGVD of } \\
\text { 1929) }\end{array}$ & $\begin{array}{l}\text { Date of } \\
\text { measure- } \\
\text { ment }\end{array}$ \\
\hline \multicolumn{9}{|c|}{ Chicot County-Continued } \\
\hline 14S03W07BBD1 & 333011 & 912620 & USGS & 77 & 134 & 27.62 & 106 & $03 / 11 / 2008$ \\
\hline 14S03W32CDB2 & 332613 & 912551 & USGS & 90 & 134 & 35.76 & 98 & $03 / 11 / 2008$ \\
\hline 15S02W20DDC1* & 332227 & 911920 & NRCS & 70 & 126 & 28.12 & 98 & $03 / 11 / 2008$ \\
\hline 15S02W20DDC1* & 332227 & 911920 & NRCS & 70 & 126 & 32 & 94 & $03 / 26 / 2008$ \\
\hline 16S03W11ADC1 & 331920 & 912234 & NRCS & -- & 118 & 29.61 & 88 & $03 / 11 / 2008$ \\
\hline 17S01E17CDA1 & 331259 & 910716 & USGS & 110 & 118 & 21 & 97 & $03 / 11 / 2008$ \\
\hline 17S01E18ADA1 & 331326 & 910758 & USGS & -- & 121 & 11.53 & 109 & $03 / 11 / 2008$ \\
\hline 17S01W06BCC1 & 331501 & 911505 & USGS & 100 & 115 & 21.57 & 93 & $03 / 11 / 2008$ \\
\hline 17S02W10AAA1 & 331429 & 911712 & USGS & 90 & 114 & 27.36 & 87 & $03 / 11 / 2008$ \\
\hline 17S03W18CBC1 & 331257 & 912736 & NRCS & -- & 117 & 35 & 82 & $03 / 26 / 2008$ \\
\hline 17S03W28DBA1 & 331127 & 912441 & USGS & 95 & 110 & 24.74 & 85 & $03 / 11 / 2008$ \\
\hline 18S01W19DAB1 & 330709 & 911423 & USGS & -- & 110 & 13.95 & 96 & $03 / 11 / 2008$ \\
\hline 18S01W33BAD1 & 330543 & 911245 & NRCS & -- & 116 & 14 & 102 & $03 / 26 / 2008$ \\
\hline 18S03W22ABA2 & 330728 & 912341 & USGS & 85.5 & 103 & 11.17 & 92 & $03 / 11 / 2008$ \\
\hline 19S01W17BCC1 & 330250 & 911406 & USGS & 120 & 106 & 20.57 & 85 & $03 / 11 / 2008$ \\
\hline 19S03W14ABB1 & 330304 & 912251 & USGS & 95 & 111 & 23.99 & 87 & $03 / 11 / 2008$ \\
\hline \multicolumn{9}{|c|}{ Clay County } \\
\hline 18N08E03DAB1 & 361323 & 901153 & USGS & 105 & 257 & 4.29 & 253 & 04/09/2008 \\
\hline 18N08E11BAA1 & 361253 & 901117 & NRCS & 100 & 259 & 4 & 255 & $04 / 02 / 2008$ \\
\hline 19N03E24AAA1 & 361655 & 904157 & USGS & -- & 278 & 20.96 & 257 & 04/09/2008 \\
\hline 19N04E11DAA1 & 361805 & 903621 & NRCS & -- & 280 & 17.8 & 262 & $04 / 02 / 2008$ \\
\hline 19N04E19AAA1 & 361654 & 904050 & USGS & -- & 282 & 31.56 & 250 & 04/09/2008 \\
\hline 19N04E19BAA1 & 361649 & 904125 & NRCS & 100 & 279 & 23.2 & 256 & $04 / 06 / 2008$ \\
\hline 19N05E15BBD1 & 361716 & 903152 & NRCS & 110 & 289 & 39 & 250 & $04 / 02 / 2008$ \\
\hline 19N06E18DBC1 & 361642 & 902815 & NRCS & -- & 297 & 38.9 & 258 & $04 / 02 / 2008$ \\
\hline 19N07E25BCB1 & 361519 & 901700 & NRCS & -- & 268 & 13.1 & 255 & $04 / 02 / 2008$ \\
\hline 19N08E08DCA1 & 361729 & 901402 & NRCS & -- & 270 & 4 & 266 & 04/02/2008 \\
\hline 19N09E19CDC1 & 361539 & 900908 & NRCS & -- & 265 & 4.5 & 261 & $04 / 02 / 2008$ \\
\hline 20N03E25BAA1 & 362112 & 904225 & NRCS & 100 & 288 & 23 & 265 & $04 / 06 / 2008$ \\
\hline 20N04E03ADA1 & 362425 & 903725 & NRCS & -- & 290 & 16 & 274 & $04 / 08 / 2008$ \\
\hline 20N05E22CAD1 & 362118 & 903132 & NRCS & -- & 290 & 29.5 & 261 & $04 / 02 / 2008$ \\
\hline 20N05E30CAC1 & 362003 & 903454 & NRCS & -- & 283 & 17.4 & 266 & 04/06/2008 \\
\hline 20N05E34DBA1 & 361939 & 903117 & USGS & 110 & 285 & 31.78 & 253 & 04/09/2008 \\
\hline 20N06E09BBA1 & 362327 & 902620 & NRCS & -- & 290 & 22.5 & 268 & $03 / 26 / 2008$ \\
\hline 20N06E28CCD1 & 362005 & 902630 & NRCS & -- & 290 & 30.8 & 259 & 44/02/2008 \\
\hline
\end{tabular}


Appendix 1. Information pertaining to water levels measured in wells completed in the Mississippi River Valley alluvial aquifer in eastern Arkansas, spring 2008.-Continued

[USGS, U.S. Geological Survey; NRCS, Natural Resources Conservation Service, ANRC, Arkansas Natural Resources Commission; --, no data; NGVD of 1929, National Geodetic Vertical Datum of 1929; Horizontal coordinate information is referenced to the North American Datum of 1983 (NAD 83); *, control wells for duplicate measurements by USGS and NRCS for quality assurance]

\begin{tabular}{|c|c|c|c|c|c|c|c|c|}
\hline Station nme & $\begin{array}{l}\text { Latitude } \\
\text { (degrees, } \\
\text { minutes, } \\
\text { seconds) }\end{array}$ & $\begin{array}{c}\text { Longitude } \\
\text { (degrees, } \\
\text { minutes, } \\
\text { seconds) }\end{array}$ & $\begin{array}{l}\text { Source } \\
\text { of data }\end{array}$ & $\begin{array}{c}\text { Depth of } \\
\text { well } \\
\text { (feet) }\end{array}$ & $\begin{array}{l}\text { Land-surface } \\
\text { datum altitude } \\
\text { (feet above } \\
\text { NGVD of } \\
\text { 1929) }\end{array}$ & $\begin{array}{c}\text { Depth to } \\
\text { water } \\
\text { (feet below } \\
\text { land-surface } \\
\text { datum) }\end{array}$ & $\begin{array}{l}\text { Water-level } \\
\text { altitude } \\
\text { (feet above } \\
\text { NGVD of } \\
\text { 1929) }\end{array}$ & $\begin{array}{c}\text { Date of } \\
\text { measure- } \\
\text { ment }\end{array}$ \\
\hline \multicolumn{9}{|c|}{ Clay County-Continued } \\
\hline 20N08E22BDC1 & 362111 & 901220 & NRCS & -- & 275 & 6.5 & 269 & $04 / 02 / 2008$ \\
\hline 20N08E24DDA1 & 362057 & 900934 & USGS & 110 & 276 & 6.16 & 270 & 04/09/2008 \\
\hline 20N09E09ABC1 & 362306 & 900642 & NRCS & -- & 279 & 3 & 276 & 04/02/2008 \\
\hline 20N09E33DDC1 & 361904 & 900628 & NRCS & -- & 270 & 5 & 265 & 04/02/2008 \\
\hline 21N03E15CBC1 & 362738 & 904453 & NRCS & 90 & 292 & 5 & 287 & 04/28/2008 \\
\hline 21N03E36CDD1 & 362450 & 904214 & NRCS & -- & 290 & 11.1 & 279 & 04/08/2008 \\
\hline 21N04E09DBC1 & 362828 & 903853 & NRCS & -- & 291 & 10 & 281 & 04/08/2008 \\
\hline 21N05E17ABB1 & 362755 & 903329 & USGS & 105 & 298 & 23.92 & 274 & 04/09/2008 \\
\hline 21N05E22BAB1 & 362704 & 903132 & NRCS & 105 & 288 & 5.8 & 282 & 04/08/2008 \\
\hline 21N06E11BBB1 & 362839 & 902421 & NRCS & 100 & 296 & 15.2 & 281 & $04 / 02 / 2008$ \\
\hline 21N06E28BB1 & 362605 & 902608 & ANRC & 130 & 292.5 & 19.38 & 273 & 04/09/2008 \\
\hline 21N07E01DDC1 & 362835 & 901607 & NRCS & 90 & 303 & 33.2 & 270 & 04/02/2008 \\
\hline 21N07E19BDA1 & 362640 & 902148 & NRCS & -- & 295 & 24.8 & 270 & 03/26/2008 \\
\hline 21N08E03CDB1 & 362848 & 901217 & NRCS & -- & 308 & 20.5 & 288 & 04/02/2008 \\
\hline 21N08E18CCC1 & 362651 & 901550 & ANRC & 110 & 324 & 40.71 & 283 & 04/09/2008 \\
\hline 21N08E36ABB1 & 362502 & 900958 & USGS & 90 & 283 & 2.68 & 280 & 04/09/2008 \\
\hline 21N09E31BDA1 & 362447 & 900851 & NRCS & 100 & 284 & 1 & 283 & 04/02/2008 \\
\hline \multicolumn{9}{|c|}{ Craighead County } \\
\hline 13N01E03AAA1 & 354739 & 905753 & NRCS & 135 & 240 & 56.6 & 183 & $03 / 17 / 2008$ \\
\hline 13N01E21CAB & 354434 & 905945 & NRCS & 120 & 240 & 65.5 & 175 & 03/17/2008 \\
\hline 13N01E23CAB1 & 354430 & 905736 & NRCS & 118 & 245 & 70.1 & 175 & 03/17/2008 \\
\hline 13N01E23DAA1 & 354435 & 905652 & USGS & 118 & 242 & 71.08 & 171 & $04 / 10 / 2008$ \\
\hline 13N02E02AAB1 & 354731 & 905032 & NRCS & 130 & 251 & 93.8 & 157 & 03/17/2008 \\
\hline 13N02E03AAA1 & 354733 & 905129 & NRCS & 105 & 250 & 88.7 & 161 & 03/17/2008 \\
\hline 13N03E23CDA1 & 354419 & 904434 & NRCS & 135 & 249 & 81.7 & 167 & $03 / 12 / 2008$ \\
\hline 13N03E29AAA1 & 354403 & 904713 & USGS & 122 & 251 & 105.76 & 145 & 04/10/2008 \\
\hline 13N03E35AAA1 & 354308 & 904401 & NRCS & 150 & 249 & 97.5 & 152 & 03/12/2008 \\
\hline 13N04E12ABB1 & 354635 & 903656 & USGS & 110 & 231 & 24.51 & 206 & 04/10/2008 \\
\hline 13N04E15DBA1 & 354521 & 903857 & NRCS & 130 & 230 & 26.6 & 203 & 03/12/2008 \\
\hline 13N04E26BCC1 & 354340 & 903829 & NRCS & 100 & 225 & 26.6 & 198 & $03 / 12 / 2008$ \\
\hline 13N05E02CCC1 & 354648 & 903202 & NRCS & 120 & 230 & 13.5 & 217 & 03/12/2008 \\
\hline 13N05E06DCC1 & 354637 & 903547 & NRCS & 110 & 229 & 20.4 & 209 & $03 / 12 / 2008$ \\
\hline 13N05E22BAD1 & 354449 & 903243 & USGS & -- & 226 & 13.23 & 213 & 04/10/2008 \\
\hline 13N05E24BAC1 & 354451 & 903045 & NRCS & 120 & 225 & 8.8 & 216 & 03/12/2008 \\
\hline
\end{tabular}


Appendix 1. Information pertaining to water levels measured in wells completed in the Mississippi River Valley alluvial aquifer in eastern Arkansas, spring 2008.-Continued

[USGS, U.S. Geological Survey; NRCS, Natural Resources Conservation Service, ANRC, Arkansas Natural Resources Commission; --, no data; NGVD of 1929, National Geodetic Vertical Datum of 1929; Horizontal coordinate information is referenced to the North American Datum of 1983 (NAD 83 ); *, control wells for duplicate measurements by USGS and NRCS for quality assurance]

\begin{tabular}{|c|c|c|c|c|c|c|c|c|}
\hline Station nme & $\begin{array}{l}\text { Latitude } \\
\text { (degrees, } \\
\text { minutes, } \\
\text { seconds) }\end{array}$ & $\begin{array}{l}\text { Longitude } \\
\text { (degrees, } \\
\text { minutes, } \\
\text { seconds) }\end{array}$ & $\begin{array}{l}\text { Source } \\
\text { of data }\end{array}$ & $\begin{array}{c}\text { Depth of } \\
\text { well } \\
\text { (feet) }\end{array}$ & $\begin{array}{l}\text { Land-surface } \\
\text { datum altitude } \\
\text { (feet above } \\
\text { NGVD of } \\
\text { 1929) }\end{array}$ & $\begin{array}{c}\text { Depth to } \\
\text { water } \\
\text { (feet below } \\
\text { land-surface } \\
\text { datum) }\end{array}$ & $\begin{array}{l}\text { Water-level } \\
\text { altitude } \\
\text { (feet above } \\
\text { NGVD of } \\
\text { 1929) }\end{array}$ & $\begin{array}{c}\text { Date of } \\
\text { measure- } \\
\text { ment }\end{array}$ \\
\hline \multicolumn{9}{|c|}{ Craighead County-Continued } \\
\hline 13N06E21AAA1 & 354450 & 902701 & NRCS & 150 & 222 & 8.5 & 214 & 03/12/2008 \\
\hline 13N07E02CAB1 & 354642 & 901901 & NRCS & 120 & 226 & 10.5 & 216 & 03/12/2008 \\
\hline 13N07E05ABB1 & 354716 & 902158 & NRCS & 100 & 225 & 4.4 & 221 & 03/12/2008 \\
\hline 13N07E20BBA1 & 354440 & 902216 & USGS & 22.3 & 223.2 & 1.95 & 221 & 04/10/2008 \\
\hline 13N07E35BCD1 & 354233 & 901837 & NRCS & 120 & 221 & 12.9 & 208 & 03/12/2008 \\
\hline 14N01E03ACB1 & 355246 & 905816 & NRCS & 96 & 249 & 51.5 & 198 & 03/17/2008 \\
\hline 14N01E10BAB1 & 355204 & 905828 & NRCS & 96 & 246 & 54 & 192 & 03/17/2008 \\
\hline 14N01E31DCA1 & 354817 & 910121 & NRCS & 126 & 251 & 61.9 & 189 & 03/17/2008 \\
\hline 14N02E18BDD1 & 355041 & 905419 & USGS & 120 & 242 & 53.57 & 188 & 04/10/2008 \\
\hline 14N02E22AAA1 & 355007 & 905129 & NRCS & 132 & 255 & 78 & 177 & 03/17/2008 \\
\hline 14N05E25ABB1 & 354921 & 903025 & USGS & -- & 238 & 18.58 & 219 & 04/10/2008 \\
\hline 14N06E06BAA1 & 355234 & 902934 & NRCS & 120 & 240 & 21.5 & 219 & 03/12/2008 \\
\hline 14N06E27AAB1 & 354911 & 902559 & USGS & 30.3 & 225.93 & -0.25 & 226 & 04/10/2008 \\
\hline 14N07E14DDC1 & 354956 & 901831 & NRCS & 120 & 230 & 13.1 & 217 & $03 / 12 / 2008$ \\
\hline 14N07E26DBB1 & 354834 & 901843 & USGS & 100 & 228 & 2.68 & 225 & 04/10/2008 \\
\hline 15N02E12DCB1 & 355626 & 904930 & NRCS & 120 & 250 & 36.5 & 214 & 03/17/2008 \\
\hline 15N03E19ADA1 & 355502 & 904802 & USGS & 116 & 262 & 51.27 & 211 & 04/10/2008 \\
\hline 15N03E31ADA1 & 355314 & 904807 & ANRC & 150 & 270 & 59.2 & 211 & 04/10/2008 \\
\hline 15N05E22BAB1 & 355513 & 903241 & NRCS & 197 & 260 & 31.8 & 228 & 03/12/2008 \\
\hline 15N06E04BAD1 & 355744 & 902706 & NRCS & 104 & 239 & 17.4 & 222 & $03 / 12 / 2008$ \\
\hline 15N06E20DDD1 & 355426 & 902739 & USGS & -- & 234 & 9.37 & 225 & 04/10/2008 \\
\hline 15N07E35DCB1 & 355241 & 901831 & NRCS & 120 & 231 & 13.9 & 217 & 03/12/2008 \\
\hline \multicolumn{9}{|c|}{ Crittenden County } \\
\hline 03N07E01ACC2 & 345349 & 901837 & USGS & 150 & 200 & 14.19 & 186 & 04/03/2008 \\
\hline 03N07E01DBB1 & 345342 & 901834 & USGS & 150 & 200 & 12.45 & 188 & 04/03/2008 \\
\hline 03N07E01DBB2 & 345343 & 901836 & USGS & 150 & 200 & 12.23 & 188 & $04 / 03 / 2008$ \\
\hline 03N07E01DBC2 & 345339 & 901839 & USGS & 150 & 203 & 16.14 & 187 & 04/03/2008 \\
\hline 03N07E01DBD1 & 345339 & 901834 & USGS & 145 & 201 & 11.21 & 190 & 04/03/2008 \\
\hline 03N07E01DBD2 & 345339 & 901832 & USGS & -- & 201 & 9.81 & 191 & 04/03/2008 \\
\hline 04N07E21AAD1 & 345644 & 902121 & USGS & 82.1 & 202 & 10.1 & 192 & 04/03/2008 \\
\hline 05N07E08BDC1 & 350407 & 902234 & NRCS & 110 & 204 & 26 & 178 & 04/07/2008 \\
\hline 05N07E28CBA1 & 350121 & 902140 & USGS & -- & 201 & 17.08 & 184 & 04/03/2008 \\
\hline 05N07E34BAB1 & 350059 & 902030 & USGS & 100 & 203 & 16.27 & 187 & 04/03/2008 \\
\hline 05N07E34CDD1 & 350010 & 902028 & NRCS & 110 & 205 & 9.9 & 195 & 04/07/2008 \\
\hline 05N08E11CCD2 & 350345 & 901308 & USGS & 63 & 211 & 25.27 & 186 & 04/03/2008 \\
\hline
\end{tabular}


Appendix 1. Information pertaining to water levels measured in wells completed in the Mississippi River Valley alluvial aquifer in eastern Arkansas, spring 2008.-Continued

[USGS, U.S. Geological Survey; NRCS, Natural Resources Conservation Service, ANRC, Arkansas Natural Resources Commission; --, no data; NGVD of 1929, National Geodetic Vertical Datum of 1929; Horizontal coordinate information is referenced to the North American Datum of 1983 (NAD 83); *, control wells for duplicate measurements by USGS and NRCS for quality assurance]

\begin{tabular}{|c|c|c|c|c|c|c|c|c|}
\hline Station nme & $\begin{array}{l}\text { Latitude } \\
\text { (degrees, } \\
\text { minutes, } \\
\text { seconds) }\end{array}$ & $\begin{array}{c}\text { Longitude } \\
\text { (degrees, } \\
\text { minutes, } \\
\text { seconds) }\end{array}$ & $\begin{array}{l}\text { Source } \\
\text { of data }\end{array}$ & $\begin{array}{c}\text { Depth of } \\
\text { well } \\
\text { (feet) }\end{array}$ & $\begin{array}{c}\text { Land-surface } \\
\text { datum altitude } \\
\text { (feet above } \\
\text { NGVD of } \\
\text { 1929) }\end{array}$ & $\begin{array}{c}\text { Depth to } \\
\text { water } \\
\text { (feet below } \\
\text { land-surface } \\
\text { datum) }\end{array}$ & $\begin{array}{l}\text { Water-level } \\
\text { altitude } \\
\text { (feet above } \\
\text { NGVD of } \\
\text { 1929) }\end{array}$ & $\begin{array}{c}\text { Date of } \\
\text { measure- } \\
\text { ment }\end{array}$ \\
\hline \multicolumn{9}{|c|}{ Crittenden County—Continued } \\
\hline 06N07E14ABA1 & 350848 & 901858 & NRCS & 110 & 211 & 23.6 & 187 & 04/07/2008 \\
\hline 07N06E29CBC1 & 351152 & 902914 & NRCS & 120 & 210 & 40.11 & 170 & 04/08/2008 \\
\hline 07N07E31CCC1 & 351042 & 902359 & USGS & 110 & 207 & 36.48 & 171 & 04/03/2008 \\
\hline 07N08E04BBD1 & 351538 & 901505 & NRCS & 120 & 224 & 22.9 & 201 & 03/26/2008 \\
\hline 07N09E05CDD1 & 351453 & 900934 & USGS & 120 & 214 & 14.83 & 199 & 04/03/2008 \\
\hline 08N06E01DCC1 & 352021 & 902408 & NRCS & 120 & 215 & 33.1 & 182 & 03/26/2008 \\
\hline 08N07E13CCC2 & 351828 & 901812 & ANRC & 100 & 221 & 31.43 & 190 & 04/03/2008 \\
\hline 08N07E14DAA2 & 351854 & 901833 & USGS & -- & 219 & 31.82 & 187 & 04/03/2008 \\
\hline 08N07E32DAA1 & 351618 & 902146 & NRCS & 110 & 215 & 31.4 & 184 & 04/07/2008 \\
\hline 08N08E06ABB1 & 352103 & 901644 & NRCS & 110 & 223 & 31.3 & 192 & 03/26/2008 \\
\hline 09N06E30ADD1 & 352235 & 902904 & NRCS & -- & 216 & 34 & 182 & 03/26/2008 \\
\hline 09N07E02CDB1 & 352537 & 901905 & NRCS & 130 & 225 & 33.5 & 192 & 03/26/2008 \\
\hline 09N07E10DDA1 & 352448 & 901925 & USGS & 60 & 221 & 29.29 & 192 & 04/03/2008 \\
\hline 09N07E31BAB1* & 352160 & 902327 & NRCS & 110 & 221 & 32.1 & 189 & 03/26/2008 \\
\hline 09N07E31BAB1* & 352160 & 902327 & NRCS & 110 & 221 & 34.06 & 187 & 04/03/2008 \\
\hline 09N08E04CDC1 & 352527 & 901444 & NRCS & 120 & 225 & 27 & 198 & 03/26/2008 \\
\hline \multicolumn{9}{|c|}{ Cross County } \\
\hline 06N02E11BDB1 & 350934 & 905132 & NRCS & -- & 220 & 69.5 & 151 & $04 / 14 / 2008$ \\
\hline 06N02E12AAA1 & 350934 & 904952 & NRCS & -- & 235 & 81 & 154 & $04 / 14 / 2008$ \\
\hline 06N04E01BBB1 & 351044 & 903739 & NRCS & -- & 205 & 37 & 168 & 04/08/2008 \\
\hline 06N05E05AAA1 & 351042 & 903432 & NRCS & 130 & 205 & 41 & 164 & 04/07/2008 \\
\hline 07N01E05CDA1 & 351518 & 910049 & USGS & 140 & 217 & 76.38 & 141 & 04/04/2008 \\
\hline 07N01E05DCA1 & 351514 & 910033 & NRCS & 160 & 215 & 77.5 & 138 & 04/08/2008 \\
\hline 07N01E06CAA1 & 351530 & 910154 & NRCS & -- & 220 & 75 & 145 & 04/08/2008 \\
\hline 07N01E11AAA1 & 351501 & 905705 & USGS & 120 & 217 & 78.62 & 138 & 04/02/2008 \\
\hline 07N02E02BBB1 & 351601 & 905144 & NRCS & -- & 227 & 76.5 & 151 & 04/08/2008 \\
\hline 07N02E10ABB1 & 351504 & 905217 & NRCS & -- & 230 & 91 & 139 & 04/08/2008 \\
\hline 07N02E15DAA1 & 351330 & 905149 & NRCS & -- & 218 & 83 & 135 & 04/08/2008 \\
\hline 07N02E29CCC1 & 351142 & 905152 & NRCS & -- & 220 & 74.5 & 146 & 04/07/2008 \\
\hline 07N02E29DDC1* & 351138 & 905409 & USGS & 100 & 220 & 74.14 & 146 & 04/02/2008 \\
\hline 07N02E29DDC1* & 351138 & 905409 & NRCS & 100 & 220 & 72.5 & 148 & 04/08/2008 \\
\hline 07N03E05AAD1 & 351558 & 904737 & NRCS & -- & 255 & 106 & 149 & $04 / 15 / 2008$ \\
\hline 07N03E05ADA1 & 351549 & 904739 & USGS & 160 & 254 & 112.23 & 142 & 04/02/2008 \\
\hline 07N03E32DCC1 & 351045 & 904810 & USGS & -- & 251 & 97.8 & 153 & $04 / 02 / 2008$ \\
\hline 07N04E07AAA1 & 351510 & 904207 & NRCS & -- & 223 & 48 & 175 & 04/08/2008 \\
\hline
\end{tabular}


Appendix 1. Information pertaining to water levels measured in wells completed in the Mississippi River Valley alluvial aquifer in eastern Arkansas, spring 2008.-Continued

[USGS, U.S. Geological Survey; NRCS, Natural Resources Conservation Service, ANRC, Arkansas Natural Resources Commission; --, no data; NGVD of 1929, National Geodetic Vertical Datum of 1929; Horizontal coordinate information is referenced to the North American Datum of 1983 (NAD 83 ); *, control wells for duplicate measurements by USGS and NRCS for quality assurance]

\begin{tabular}{|c|c|c|c|c|c|c|c|c|}
\hline Station nme & $\begin{array}{c}\text { Latitude } \\
\text { (degrees, } \\
\text { minutes, } \\
\text { seconds) }\end{array}$ & $\begin{array}{l}\text { Longitude } \\
\text { (degrees, } \\
\text { minutes, } \\
\text { seconds) }\end{array}$ & $\begin{array}{l}\text { Source } \\
\text { of data }\end{array}$ & $\begin{array}{c}\text { Depth of } \\
\text { well } \\
\text { (feet) }\end{array}$ & $\begin{array}{l}\text { Land-surface } \\
\text { datum altitude } \\
\text { (feet above } \\
\text { NGVD of } \\
\text { 1929) }\end{array}$ & $\begin{array}{c}\text { Depth to } \\
\text { water } \\
\text { (feet below } \\
\text { land-surface } \\
\text { datum) }\end{array}$ & $\begin{array}{l}\text { Water-leve } \\
\text { altitude } \\
\text { (feet above } \\
\text { NGVD of } \\
\text { 1929) }\end{array}$ & $\begin{array}{c}\text { Date of } \\
\text { measure- } \\
\text { ment }\end{array}$ \\
\hline \multicolumn{9}{|c|}{ Cross County-Continued } \\
\hline 07N04E27BDA1 & 351220 & 903926 & NRCS & -- & 203 & 24.5 & 179 & 04/07/2008 \\
\hline 07N05E02AAB1 & 351600 & 903103 & NRCS & -- & 210 & 43 & 167 & 04/08/2008 \\
\hline 07N05E16ACA1 & 351358 & 903352 & NRCS & -- & 210 & 31.5 & 179 & 04/08/2008 \\
\hline 07N05E19CCC1 & 351238 & 903645 & USGS & -- & 207 & 39.34 & 168 & 04/03/2008 \\
\hline 07N05E24CCC1 & 351232 & 903121 & NRCS & 110 & 205 & 37.6 & 167 & 04/07/2008 \\
\hline 07N05E25ABA1 & 351229 & 903045 & NRCS & 140 & 205 & 38.94 & 166 & 04/03/2008 \\
\hline 08N01E02CDD1 & 352023 & 905736 & NRCS & -- & 226 & 89 & 137 & 04/08/2008 \\
\hline 08N01E16DBB1 & 351855 & 905933 & NRCS & 140 & 225 & 87 & 138 & 04/08/2008 \\
\hline 08N01E17CAD1 & 351852 & 910046 & NRCS & -- & 220 & 76 & 144 & 04/08/2008 \\
\hline 08N02E12DCC1 & 351938 & 905002 & NRCS & -- & 230 & 92 & 138 & 04/14/2008 \\
\hline 08N02E17AAA1 & 351923 & 905354 & NRCS & -- & 225 & 90 & 135 & 04/08/2008 \\
\hline 08N02E29CBA1 & 351715 & 905438 & NRCS & -- & 225 & 86.5 & 139 & 04/08/2008 \\
\hline 08N03E15BBB1 & 351942 & 904620 & NRCS & -- & 265 & 115 & 150 & 04/14/2008 \\
\hline 08N05E17CAA1 & 351904 & 903508 & NRCS & -- & 211 & 32 & 179 & 04/08/2008 \\
\hline 08N05E32ADD1 & 351632 & 903440 & USGS & -- & 204 & 31.47 & 173 & 04/03/2008 \\
\hline 09N01E04ACD1 & 352608 & 905914 & NRCS & 140 & 225 & 90 & 135 & 04/08/2008 \\
\hline 09N01E33BBA2 & 352203 & 910001 & USGS & -- & 225 & 83.67 & 141 & 04/04/2008 \\
\hline 09N01E36AAB1 & 352155 & 905605 & NRCS & 160 & 225 & 91.5 & 134 & 04/08/2008 \\
\hline 09N02E20AAA1 & 352402 & 905342 & NRCS & 120 & 231 & 94 & 137 & 04/08/2008 \\
\hline 09N02E30CBB1 & 352243 & 905551 & NRCS & -- & 225 & 90 & 135 & 04/08/2008 \\
\hline 09N02E32BBB1 & 352213 & 905444 & NRCS & -- & 226 & 100 & 126 & 04/08/2008 \\
\hline 09N03E03ACA1 & 352630 & 904529 & NRCS & & 250 & 108 & 142 & 04/14/2008 \\
\hline 09N03E17CDD1 & 352422 & 904753 & NRCS & -- & 245 & 105.5 & 140 & 04/14/2008 \\
\hline 09N03E17DDC1 & 352409 & 904726 & USGS & 160 & 251 & 107.77 & 143 & 04/04/2008 \\
\hline 09N05E10ACC1 & 352511 & 903249 & NRCS & 120 & 210 & 20 & 190 & 04/09/2008 \\
\hline 09N05E32BCB1 & 352151 & 903525 & NRCS & -- & 206 & 30 & 176 & 04/09/2008 \\
\hline 09N05E32BDB1 & 352151 & 903512 & USGS & -- & 210 & 32.17 & 178 & 04/03/2008 \\
\hline \multicolumn{9}{|c|}{ Desha County } \\
\hline 07S01E19ABA1 & 340428 & 910303 & NRCS & 120 & 154 & 22.5 & 132 & $03 / 25 / 2008$ \\
\hline 08S03W33ABD1 & 335803 & 912338 & USGS & 60 & 165.04 & 6.93 & 158 & 03/13/2008 \\
\hline 09S01W08BDA1 & 335608 & 911234 & NRCS & -- & 156 & 30 & 126 & 04/01/2008 \\
\hline 09S01W15CBB1 & 335501 & 911055 & NRCS & -- & 152 & 39 & 113 & 04/01/2008 \\
\hline 09S02W26DDC1 & 335257 & 911530 & USGS & 97 & 149.27 & 31.68 & 118 & 05/13/2008 \\
\hline 09S03W05BAC1 & 335704 & 912506 & NRCS & -- & 161 & 36 & 125 & 04/01/2008 \\
\hline 09S03W13BAB1 & 335500 & 911922 & NRCS & -- & 156 & 35 & 121 & 04/01/2008 \\
\hline
\end{tabular}


Appendix 1. Information pertaining to water levels measured in wells completed in the Mississippi River Valley alluvial aquifer in eastern Arkansas, spring 2008.-Continued

[USGS, U.S. Geological Survey; NRCS, Natural Resources Conservation Service, ANRC, Arkansas Natural Resources Commission; --, no data; NGVD of 1929, National Geodetic Vertical Datum of 1929; Horizontal coordinate information is referenced to the North American Datum of 1983 (NAD 83); *, control wells for duplicate measurements by USGS and NRCS for quality assurance]

\begin{tabular}{|c|c|c|c|c|c|c|c|c|}
\hline Station nme & $\begin{array}{l}\text { Latitude } \\
\text { (degrees, } \\
\text { minutes, } \\
\text { seconds) }\end{array}$ & $\begin{array}{l}\text { Longitude } \\
\text { (degrees, } \\
\text { minutes, } \\
\text { seconds) }\end{array}$ & $\begin{array}{l}\text { Source } \\
\text { of data }\end{array}$ & $\begin{array}{c}\text { Depth of } \\
\text { well } \\
\text { (feet) }\end{array}$ & $\begin{array}{l}\text { Land-surface } \\
\text { datum altitude } \\
\text { (feet above } \\
\text { NGVD of } \\
\text { 1929) }\end{array}$ & $\begin{array}{c}\text { Depth to } \\
\text { water } \\
\text { (feet below } \\
\text { land-surface } \\
\text { datum) }\end{array}$ & $\begin{array}{l}\text { Water-leve } \\
\text { altitude } \\
\text { (feet above } \\
\text { NGVD of } \\
\text { 1929) }\end{array}$ & $\begin{array}{c}\text { Date of } \\
\text { measure- } \\
\text { ment }\end{array}$ \\
\hline \multicolumn{9}{|c|}{ Desha County-Continued } \\
\hline 09S03W17DCB1 & 335448 & 912457 & USGS & 126 & 155.08 & 33.68 & 121 & 03/13/2008 \\
\hline 09S04W06BCA1 & 335756 & 913243 & USGS & -- & 161 & 36.43 & 125 & 03/13/2008 \\
\hline 10S01W23CDA1 & 335305 & 911032 & NRCS & -- & 151 & 31 & 120 & 04/01/2008 \\
\hline 10S02W11ADD1 & 335045 & 911517 & NRCS & -- & 146 & 31 & 115 & 04/01/2008 \\
\hline 10S02W20ADA1 & 334916 & 911825 & ANRC & 93.8 & 148 & 40.85 & 107 & 03/12/2008 \\
\hline 10S02W24DBC1 & 334850 & 911453 & USGS & 70 & 143 & 26.18 & 117 & $03 / 12 / 2008$ \\
\hline 10S03W26CAA1 & 334806 & 912145 & USGS & 96 & 155 & 47.43 & 108 & 03/12/2008 \\
\hline 10S04W03ABB1 & 335208 & 912931 & ANRC & 100 & 165 & 36.2 & 129 & 03/13/2008 \\
\hline 10S04W03BAB1 & 335209 & 912948 & USGS & 100 & 166 & 36.86 & 129 & 03/13/2008 \\
\hline 10S04W11DDA1 & 335031 & 912802 & ANRC & 100 & 155 & 33.33 & 122 & 03/13/2008 \\
\hline 10S04W12DBB1 & 335102 & 912729 & ANRC & 99 & 152 & 31.73 & 120 & 03/13/2008 \\
\hline 11S02W15ADD1 & 334446 & 911635 & NRCS & -- & 144 & 36 & 108 & 04/01/2008 \\
\hline 11S03W16CBA1 & 334439 & 912433 & NRCS & -- & 155 & 36 & 119 & 04/01/2008 \\
\hline 11S03W31BBA1 & 334228 & 912651 & USGS & -- & 148 & 37.27 & 111 & $03 / 12 / 2008$ \\
\hline 12S01W33BAA1 & 333718 & 911205 & USGS & 95 & 135 & 25.21 & 110 & 03/13/2008 \\
\hline 13S02W17ADA1 & 333421 & 911858 & NRCS & -- & 138 & 46 & 92 & 04/01/2008 \\
\hline $13 \mathrm{~S} 02 \mathrm{~W} 27 \mathrm{CAC} 1$ & 333224 & 911735 & USGS & 120 & 133 & 32.43 & 101 & $03 / 12 / 2008$ \\
\hline 13S02W32DBD1 & 333126 & 911917 & NRCS & -- & 135 & 40 & 95 & 04/01/2008 \\
\hline 13S03W10DAA1 & 333506 & 912302 & USGS & 86 & 140 & 49.17 & 91 & $03 / 12 / 2008$ \\
\hline 13S03W11CAB1 & 333503 & 912241 & NRCS & -- & 142 & 52 & 90 & 04/01/2008 \\
\hline \multicolumn{9}{|c|}{ Drew County } \\
\hline 11S04W08DBA1 & 334532 & 913136 & USGS & 70 & 160 & 26.08 & 134 & $03 / 12 / 2008$ \\
\hline 11S05W08CCC1 & 334546 & 913837 & USGS & 153 & 185 & 37.47 & 148 & $03 / 12 / 2008$ \\
\hline 12S04W03ABB1 & 334134 & 912946 & USGS & -- & 155 & 26.02 & 129 & $03 / 12 / 2008$ \\
\hline 12S04W25DBB1 & 333739 & 912738 & NRCS & 90 & 149 & 36 & 113 & 03/17/2008 \\
\hline 13S04W09ACD1 & 333512 & 913034 & NRCS & 90 & 145 & 20 & 125 & 03/17/2008 \\
\hline 13S04W28CDD1 & 333206 & 913100 & USGS & 65 & 139 & 19.92 & 119 & 03/12/2008 \\
\hline 13S04W33BAA1 & 333206 & 913100 & USGS & 130 & 138 & 19.32 & 119 & $03 / 12 / 2008$ \\
\hline 13S05W29ADA1 & 333248 & 913747 & USGS & -- & 185 & 46.85 & 138 & $03 / 12 / 2008$ \\
\hline 13S06W03DDC1 & 333545 & 914202 & USGS & 110 & 191 & 64.89 & 126 & 03/12/2008 \\
\hline 13S06W21DAA1 & 333324 & 914258 & NRCS & 142 & 207 & 72 & 135 & 03/17/2008 \\
\hline 14S04W03ADD1 & 333050 & 912929 & NRCS & 92 & 141 & 28 & 113 & 03/17/2008 \\
\hline 14S04W05CBC1 & 333042 & 913226 & NRCS & 90 & 131 & 20 & 111 & 03/17/2008 \\
\hline 15S04W13DAD1 & 332338 & 912730 & NRCS & -- & 131 & 42 & 89 & 03/25/2008 \\
\hline
\end{tabular}


Appendix 1. Information pertaining to water levels measured in wells completed in the Mississippi River Valley alluvial aquifer in eastern Arkansas, spring 2008.-Continued

[USGS, U.S. Geological Survey; NRCS, Natural Resources Conservation Service, ANRC, Arkansas Natural Resources Commission; --, no data; NGVD of 1929, National Geodetic Vertical Datum of 1929; Horizontal coordinate information is referenced to the North American Datum of 1983 (NAD 83 ); *, control wells for duplicate measurements by USGS and NRCS for quality assurance]

\begin{tabular}{|c|c|c|c|c|c|c|c|c|}
\hline Station nme & $\begin{array}{l}\text { Latitude } \\
\text { (degrees, } \\
\text { minutes, } \\
\text { seconds) }\end{array}$ & $\begin{array}{l}\text { Longitude } \\
\text { (degrees, } \\
\text { minutes, } \\
\text { seconds) }\end{array}$ & $\begin{array}{l}\text { Source } \\
\text { of data }\end{array}$ & $\begin{array}{c}\text { Depth of } \\
\text { well } \\
\text { (feet) }\end{array}$ & $\begin{array}{l}\text { Land-surface } \\
\text { datum altitude } \\
\text { (feet above } \\
\text { NGVD of } \\
\text { 1929) }\end{array}$ & $\begin{array}{c}\text { Depth to } \\
\text { water } \\
\text { (feet below } \\
\text { land-surface } \\
\text { datum) }\end{array}$ & $\begin{array}{l}\text { Water-level } \\
\text { altitude } \\
\text { (feet above } \\
\text { NGVD of } \\
\text { 1929) }\end{array}$ & $\begin{array}{l}\text { Date of } \\
\text { measure- } \\
\text { ment }\end{array}$ \\
\hline \multicolumn{9}{|c|}{ Greene County } \\
\hline 16N03E03BA1 & 360316 & 904516 & ANRC & 100 & 260 & 33.17 & 227 & 04/09/2008 \\
\hline 16N03E05BBB1 & 360316 & 904750 & NRCS & 105 & 257 & 32.6 & 224 & 04/17/2008 \\
\hline 16N03E16DDD1 & 360049 & 904547 & NRCS & 100 & 258 & 28.1 & 230 & $04 / 17 / 2008$ \\
\hline 16N03E29ACC1 & 355926 & 904722 & NRCS & 100 & 257 & 37.1 & 220 & $04 / 17 / 2008$ \\
\hline 16N06E03CCC1 & 360224 & 902626 & USGS & 194 & 258 & 41.19 & 217 & $04 / 09 / 2008$ \\
\hline 16N06E09ABB1 & 360215 & 902651 & NRCS & 90 & 261 & 40.2 & 221 & 04/07/2008 \\
\hline 16N06E21BAA1 & 360031 & 902705 & NRCS & 130 & 249 & 27.6 & 221 & 04/07/2008 \\
\hline 16N06E28ABB1 & 355938 & 902657 & USGS & -- & 251 & 25.73 & 225 & $04 / 09 / 2008$ \\
\hline 17N03E02DCC1 & 360806 & 904352 & NRCS & 100 & 267 & 30.8 & 236 & $04 / 17 / 2008$ \\
\hline 17N04E07AD1 & 360718 & 904122 & NRCS & 100 & 273 & 38.1 & 235 & $04 / 17 / 2008$ \\
\hline 17N04E30CDC1 & 360409 & 904218 & USGS & 100 & 265 & 39.09 & 226 & 04/09/2008 \\
\hline 17N06E15ABC1 & 360631 & 902546 & NRCS & 168 & 268 & 30.9 & 237 & $04 / 17 / 2008$ \\
\hline 17N07E01BBA1 & 360832 & 901724 & NRCS & 100 & 250 & 5.8 & 244 & 04/07/2008 \\
\hline 17N07E18ABB1 & 360638 & 902235 & USGS & -- & 245 & 7.85 & 237 & 04/09/2008 \\
\hline 18N03E24ACA1 & 361119 & 904216 & NRCS & 120 & 271 & 35.1 & 236 & $04 / 17 / 2008$ \\
\hline 18N04E04AAC1 & 361356 & 903854 & NRCS & 127 & 273 & 31.3 & 242 & $04 / 17 / 2008$ \\
\hline 18N04E21CBD1 & 361052 & 903725 & USGS & -- & 294 & 58.57 & 235 & 04/09/2008 \\
\hline 18N06E23ABB1 & 361109 & 902402 & NRCS & 145 & 280 & 15.4 & 265 & $04 / 17 / 2008$ \\
\hline 18N07E05DAB1 & 361316 & 902025 & NRCS & -- & 274 & 12.2 & 262 & $04 / 07 / 2008$ \\
\hline 18N07E17BAB1 & 361203 & 902105 & NRCS & 100 & 262 & 8.6 & 253 & $04 / 07 / 2008$ \\
\hline 18N07E20BBA1 & 361110 & 902113 & USGS & -- & 257 & 7.23 & 250 & 04/09/2008 \\
\hline 19N03E26AD1 & 361601 & 904258 & USGS & 100 & 281 & 30.28 & 251 & $04 / 09 / 2008$ \\
\hline 19N03E33DDD1 & 361418 & 904516 & NRCS & 100 & 276 & 36.5 & 240 & $04 / 17 / 2008$ \\
\hline 19N05E34AAD1 & 361437 & 903102 & NRCS & 130 & 282 & 34.8 & 247 & $04 / 17 / 2008$ \\
\hline \multicolumn{9}{|c|}{ Independence County } \\
\hline 12N04W14DD1 & 353929 & 912236 & ANRC & 60 & 231 & 10.54 & 220 & $04 / 16 / 2008$ \\
\hline 12N04W34CBB1 & 353720 & 912513 & USGS & -- & 231 & 4.47 & 227 & $04 / 16 / 2008$ \\
\hline 12N05W36AAA1 & 353738 & 912827 & NRCS & -- & 236 & 6.41 & 230 & $04 / 16 / 2008$ \\
\hline \multicolumn{9}{|c|}{ Jackson County } \\
\hline 09N01W22ADD1 & 352332 & 910433 & USGS & 125 & 215 & 63.04 & 152 & $04 / 15 / 2008$ \\
\hline 09N02W32BBB1 & 352215 & 911344 & NRCS & 100 & 220 & 32.2 & 188 & 03/27/2008 \\
\hline 09N02W32CBB1 & 352152 & 911348 & USGS & 117 & 220 & 30.39 & 190 & $04 / 15 / 2008$ \\
\hline 10N02W29ABB1 & 352829 & 911312 & USGS & -- & 227 & 28.47 & 199 & $04 / 15 / 2008$ \\
\hline 11N01W26AAD1* & 353330 & 910323 & NRCS & 95 & 227 & 69.3 & 158 & 03/27/2008 \\
\hline 11N01W26AAD1* & 353330 & 910323 & USGS & 95 & 227 & 68.29 & 159 & $04 / 15 / 2008$ \\
\hline
\end{tabular}


Appendix 1. Information pertaining to water levels measured in wells completed in the Mississippi River Valley alluvial aquifer in eastern Arkansas, spring 2008. - Continued

[USGS, U.S. Geological Survey; NRCS, Natural Resources Conservation Service, ANRC, Arkansas Natural Resources Commission; --, no data; NGVD of 1929, National Geodetic Vertical Datum of 1929; Horizontal coordinate information is referenced to the North American Datum of 1983 (NAD 83 ); *, control wells for duplicate measurements by USGS and NRCS for quality assurance]

\begin{tabular}{|c|c|c|c|c|c|c|c|c|}
\hline Station nme & $\begin{array}{l}\text { Latitude } \\
\text { (degrees, } \\
\text { minutes, } \\
\text { seconds) }\end{array}$ & $\begin{array}{c}\text { Longitude } \\
\text { (degrees, } \\
\text { minutes, } \\
\text { seconds) }\end{array}$ & $\begin{array}{l}\text { Source } \\
\text { of data }\end{array}$ & $\begin{array}{c}\text { Depth of } \\
\text { well } \\
\text { (feet) }\end{array}$ & $\begin{array}{l}\text { Land-surface } \\
\text { datum altitude } \\
\text { (feet above } \\
\text { NGVD of } \\
\text { 1929) }\end{array}$ & $\begin{array}{c}\text { Depth to } \\
\text { water } \\
\text { (feet below } \\
\text { land-surface } \\
\text { datum) }\end{array}$ & $\begin{array}{l}\text { Water-level } \\
\text { altitude } \\
\text { (feet above } \\
\text { NGVD of } \\
\text { 1929) }\end{array}$ & $\begin{array}{c}\text { Date of } \\
\text { measure- } \\
\text { ment }\end{array}$ \\
\hline \multicolumn{9}{|c|}{ Jackson County-Continued } \\
\hline 11N01W29AAD1 & 353339 & 910635 & USGS & 97 & 225 & 41.88 & 183 & $04 / 15 / 2008$ \\
\hline 11N02W25BBD1 & 353322 & 910855 & NRCS & 100 & 221 & 28.1 & 193 & $03 / 27 / 2008$ \\
\hline 12N01W11BCB1 & 354127 & 910416 & NRCS & 110 & 233 & 40.7 & 192 & 03/27/2008 \\
\hline 12N02W25ABB2 & 353910 & 910852 & USGS & -- & 234 & 34.41 & 200 & 04/16/2008 \\
\hline 13N01W20AAA1 & 354514 & 910627 & USGS & 147 & 242 & 41.59 & 200 & 04/16/2008 \\
\hline 13N03W15CDD1 & 354526 & 911749 & USGS & -- & 232 & 7.41 & 225 & $04 / 16 / 2008$ \\
\hline 13N03W36ABB1 & 354337 & 911532 & NRCS & 110 & 241 & 16.3 & 225 & 03/27/2008 \\
\hline 14N01W08AAA1 & 355216 & 910623 & NRCS & 80 & 252 & 37.5 & 215 & 03/27/2008 \\
\hline 14N01W09AAA1 & 355220 & 910515 & USGS & -- & 251 & 43.88 & 207 & 04/16/2008 \\
\hline 14N02W22BBC1 & 355026 & 911145 & NRCS & 100 & 250 & 25.2 & 225 & 03/27/2008 \\
\hline \multicolumn{9}{|c|}{ Jefferson County } \\
\hline 03S08W24BBC1 & 342620 & 914953 & USGS & 135 & 202 & 51.03 & 151 & 03/18/2008 \\
\hline 03S09W06DDA1 & 342840 & 920037 & USGS & -- & 225 & 37.02 & 188 & 03/18/2008 \\
\hline 03S09W14BCD1 & 342712 & 915713 & NRCS & -- & 220 & 51 & 169 & 03/31/2008 \\
\hline 03S09W22AAA1 & 342640 & 915728 & NRCS & 100 & 218 & 43 & 175 & $03 / 31 / 2008$ \\
\hline 03S09W29CBD1 & 342517 & 920023 & USGS & -- & 216 & 27.74 & 188 & 03/18/2008 \\
\hline 03S09W36ACC1 & 342428 & 915555 & NRCS & -- & 214 & 29 & 185 & $03 / 31 / 2008$ \\
\hline 03S10W26AAA1 & 342551 & 920300 & NRCS & -- & 216 & 25 & 191 & 03/31/2008 \\
\hline 03S10W35BBC1 & 342449 & 920359 & NRCS & -- & 215 & 8.5 & 207 & 03/31/2008 \\
\hline 04S07W35DDB1 & 341836 & 914347 & NRCS & -- & 185 & 28.8 & 156 & 03/31/2008 \\
\hline 04S08W13DCB1 & 342123 & 914926 & USGS & 110 & 204 & 47.99 & 156 & 03/18/2008 \\
\hline 04S09W02CBD1 & 342325 & 915717 & NRCS & 110 & 212 & 26.6 & 185 & $03 / 31 / 2008$ \\
\hline 04S09W32DDA1 & 341859 & 920009 & NRCS & -- & 212 & 16 & 196 & 03/31/2008 \\
\hline 05S06W31CAA1 & 341330 & 914206 & USGS & -- & 189.22 & 18.33 & 171 & 03/18/2008 \\
\hline 05S07W29DDD1 & 341411 & 914654 & NRCS & 110 & 194 & 16.1 & 178 & $03 / 31 / 2008$ \\
\hline 05S08W12DAA1 & 341712 & 914907 & USGS & 101 & 194.25 & 16.15 & 178 & 03/18/2008 \\
\hline 06S05W15BCA1 & 341023 & 913245 & USGS & 120 & 177.14 & 18.22 & 159 & 03/18/2008 \\
\hline 06S06W23AAD1 & 341007 & 913712 & USGS & 107 & 189.01 & 19.02 & 170 & 03/18/2008 \\
\hline 06S07W14BAA1 & 341125 & 914426 & USGS & 110 & 199 & 15.25 & 184 & 03/18/2008 \\
\hline 07S08W06BAA1 & 340859 & 915647 & USGS & 160 & 202.31 & 19.07 & 183 & 03/18/2008 \\
\hline \multicolumn{9}{|c|}{ Lawrence County } \\
\hline 15N01E26DDA1 & 355402 & 905639 & USGS & 100 & 251 & 54.07 & 197 & $04 / 10 / 2008$ \\
\hline 15N01W35CBB1 & 355336 & 910356 & USGS & -- & 250 & 46.68 & 203 & 04/10/2008 \\
\hline 16N01E11DAC2 & 360203 & 905639 & USGS & -- & 262 & 48.69 & 213 & 04/10/2008 \\
\hline 16N01W30DDC1 & 355937 & 910723 & NRCS & 105 & 255 & 13 & 242 & 03/24/2008 \\
\hline
\end{tabular}


Appendix 1. Information pertaining to water levels measured in wells completed in the Mississippi River Valley alluvial aquifer in eastern Arkansas, spring 2008.-Continued

[USGS, U.S. Geological Survey; NRCS, Natural Resources Conservation Service, ANRC, Arkansas Natural Resources Commission; --, no data; NGVD of 1929, National Geodetic Vertical Datum of 1929; Horizontal coordinate information is referenced to the North American Datum of 1983 (NAD 83 ); *, control wells for duplicate measurements by USGS and NRCS for quality assurance]

\begin{tabular}{|c|c|c|c|c|c|c|c|c|}
\hline Station nme & $\begin{array}{c}\text { Latitude } \\
\text { (degrees, } \\
\text { minutes, } \\
\text { seconds) }\end{array}$ & $\begin{array}{l}\text { Longitude } \\
\text { (degrees, } \\
\text { minutes, } \\
\text { seconds) }\end{array}$ & $\begin{array}{l}\text { Source } \\
\text { of data }\end{array}$ & $\begin{array}{c}\text { Depth of } \\
\text { well } \\
\text { (feet) }\end{array}$ & $\begin{array}{l}\text { Land-surface } \\
\text { datum altitude } \\
\text { (feet above } \\
\text { NGVD of } \\
\text { 1929) }\end{array}$ & $\begin{array}{c}\text { Depth to } \\
\text { water } \\
\text { (feet below } \\
\text { land-surface } \\
\text { datum) }\end{array}$ & $\begin{array}{l}\text { Water-level } \\
\text { altitude } \\
\text { (feet above } \\
\text { NGVD of } \\
\text { 1929) }\end{array}$ & $\begin{array}{c}\text { Date of } \\
\text { measure- } \\
\text { ment }\end{array}$ \\
\hline \multicolumn{9}{|c|}{ Lawrence County-Continued } \\
\hline 16N02E34CBB1 & 355831 & 905208 & NRCS & 100 & 255 & 50 & 205 & 03/24/2008 \\
\hline 17N01E02BBA1 & 360901 & 905707 & NRCS & 90 & 260 & 16.2 & 244 & 03/24/2008 \\
\hline 17N02E04DCA1 & 360758 & 905224 & NRCS & 110 & 270 & 44.2 & 226 & 03/24/2008 \\
\hline 17N02E19CDC1 & 360516 & 905449 & NRCS & 105 & 265 & 41.07 & 224 & 04/10/2008 \\
\hline \multicolumn{9}{|c|}{ Lee County } \\
\hline 01N01E09CCC1 & 344215 & 910054 & NRCS & 140 & 182 & 35.5 & 147 & 04/15/2008 \\
\hline 01N01E24CBD1 & 344033 & 905729 & NRCS & 140 & 185 & 16.7 & 168 & $04 / 15 / 2008$ \\
\hline 01N02E01ADD1 & 344330 & 905016 & NRCS & 140 & 207 & 31 & 176 & 04/15/2008 \\
\hline 01N02E11BAB1 & 344255 & 905208 & NRCS & 140 & 202 & 33 & 169 & 04/15/2008 \\
\hline 01N02E12ABB1 & 344254 & 905040 & NRCS & 140 & 206 & 34 & 172 & 04/15/2008 \\
\hline 01N02E22CBA1 & 344056 & 905318 & NRCS & 140 & 200 & 29.5 & 171 & $04 / 15 / 2008$ \\
\hline 01N02E33CBB1 & 343858 & 905434 & NRCS & 140 & 186 & 11 & 175 & $04 / 15 / 2008$ \\
\hline 01N02E33CCB1 & 343851 & 905433 & NRCS & 140 & 185 & 9 & 176 & $04 / 15 / 2008$ \\
\hline 01N03E02BBC1 & 344339 & 904601 & USGS & 168 & 236.43 & 53.68 & 183 & 04/01/2008 \\
\hline 01N03E35BBA1 & 343923 & 904549 & USGS & 120 & 202 & 13.85 & 188 & 04/01/2008 \\
\hline 02N01E21BAA1 & 344633 & 910005 & NRCS & 140 & 185 & 38.3 & 147 & 04/15/2008 \\
\hline 02N01E23BAA2 & 344632 & 905820 & USGS & 137 & 202 & 52.1 & 150 & 04/01/2008 \\
\hline 02N01W12BAA1 & 344828 & 910330 & USGS & 95 & 185 & 45.65 & 139 & $04 / 01 / 2008$ \\
\hline 02N01W34DDC1 & 344410 & 910520 & NRCS & 140 & 180 & 52 & 128 & $04 / 15 / 2008$ \\
\hline 02N02E08ADC1 & 344807 & 905339 & ANRC & 120 & 201 & 45.92 & 155 & 04/01/2008 \\
\hline 02N02E21ABC1 & 344622 & 905358 & USGS & 120 & 200 & 41.25 & 159 & 04/01/2008 \\
\hline 02N02E22BBB1 & 344628 & 905327 & NRCS & 140 & 200 & 34 & 166 & 04/15/2008 \\
\hline 02N03E08AAD1 & 344811 & 904838 & USGS & 100 & 211 & 45.73 & 165 & 04/01/2008 \\
\hline 02N03E09DDD1 & 344723 & 904707 & NRCS & 120 & 220 & 47 & 173 & $04 / 15 / 2008$ \\
\hline 02N03E29CAD1 & 344500 & 904846 & NRCS & 140 & 220 & 43 & 177 & 04/15/2008 \\
\hline 02N04E03ABD1 & 344855 & 903954 & NRCS & 140 & 192 & 26 & 166 & $04 / 15 / 2008$ \\
\hline 02N04E15DAC1 & 344637 & 903950 & USGS & 60 & 192 & 20.67 & 171 & 04/01/2008 \\
\hline 03N01E15CCB1 & 345206 & 905947 & ANRC & -- & 205 & 63.96 & 141 & 04/01/2008 \\
\hline 03N01E16CBA1 & 345222 & 910040 & ANRC & 110 & 202 & 65.14 & 137 & 04/01/2008 \\
\hline 03N02E12CDC1 & 345239 & 905053 & NRCS & 140 & 210 & 43 & 167 & $04 / 15 / 2008$ \\
\hline 03N02E13BBA1 & 345237 & 905107 & USGS & 65 & 212 & 51.87 & 160 & $04 / 01 / 2008$ \\
\hline 03N02E21CBC1 & 345111 & 905428 & NRCS & 140 & 209 & 56 & 153 & 04/15/2008 \\
\hline 03N02E29DAD1 & 345014 & 905430 & USGS & 135 & 205 & 44.64 & 160 & 04/01/2008 \\
\hline 03N03E05CDD1 & 345327 & 904837 & NRCS & 110 & 204 & 49 & 155 & 04/15/2008 \\
\hline 03N03E32CAB1 & 344933 & 904926 & ANRC & 116 & 204 & 51.22 & 153 & 04/01/2008 \\
\hline
\end{tabular}


Appendix 1. Information pertaining to water levels measured in wells completed in the Mississippi River Valley alluvial aquifer in eastern Arkansas, spring 2008._-Continued

[USGS, U.S. Geological Survey; NRCS, Natural Resources Conservation Service, ANRC, Arkansas Natural Resources Commission; --, no data; NGVD of 1929, National Geodetic Vertical Datum of 1929; Horizontal coordinate information is referenced to the North American Datum of 1983 (NAD 83 ); *, control wells for duplicate measurements by USGS and NRCS for quality assurance]

\begin{tabular}{|c|c|c|c|c|c|c|c|c|}
\hline Station nme & $\begin{array}{c}\text { Latitude } \\
\text { (degrees, } \\
\text { minutes, } \\
\text { seconds) }\end{array}$ & $\begin{array}{l}\text { Longitude } \\
\text { (degrees, } \\
\text { minutes, } \\
\text { seconds) }\end{array}$ & $\begin{array}{l}\text { Source } \\
\text { of data }\end{array}$ & $\begin{array}{c}\text { Depth of } \\
\text { well } \\
\text { (feet) }\end{array}$ & $\begin{array}{l}\text { Land-surface } \\
\text { datum altitude } \\
\text { (feet above } \\
\text { NGVD of } \\
\text { 1929) }\end{array}$ & $\begin{array}{c}\text { Depth to } \\
\text { water } \\
\text { (feet below } \\
\text { land-surface } \\
\text { datum) }\end{array}$ & $\begin{array}{c}\text { Water-leve } \\
\text { altitude } \\
\text { (feet above } \\
\text { NGVD of } \\
\text { 1929) }\end{array}$ & $\begin{array}{c}\text { Date of } \\
\text { measure- } \\
\text { ment }\end{array}$ \\
\hline \multicolumn{9}{|c|}{ Lee County-Continued } \\
\hline 03N04E07CBB1 & 345245 & 904312 & NRCS & 140 & 200 & 30 & 170 & $04 / 15 / 2008$ \\
\hline 03N05E14DDA1 & 345148 & 903203 & ANRC & 120 & 193 & 15.79 & 177 & 04/01/2008 \\
\hline \multicolumn{9}{|c|}{ Lincoln County } \\
\hline 07S06W03CCA2 & 340828 & 914114 & NRCS & 110 & 190 & 18 & 172 & 03/12/2008 \\
\hline 07S07W36CBD1 & 340411 & 914529 & NRCS & 123 & 183 & 41 & 142 & 03/12/2008 \\
\hline 08S04W06ABD1 & 340341 & 913116 & NRCS & 95 & 171 & 16 & 155 & 03/12/2008 \\
\hline 08S04W08BBB2 & 340254 & 913101 & USGS & 65.2 & 171 & 23.23 & 148 & 03/13/2008 \\
\hline 08S04W29ABC1 & 340021 & 913044 & NRCS & 100 & 176 & 45 & 131 & 03/12/2008 \\
\hline 08S04W31CBA1 & 335901 & 913150 & USGS & 99 & 161.9 & 35.11 & 127 & 03/13/2008 \\
\hline 08S05W12AAD1 & 340246 & 913214 & NRCS & 83 & 165 & 24 & 141 & 03/12/2008 \\
\hline 08S05W21DCD1 & 340027 & 913533 & NRCS & 120 & 169 & 30 & 139 & 03/12/2008 \\
\hline 08S05W32DCC1 & 335840 & 913644 & NRCS & 100 & 172 & 47 & 125 & 03/12/2008 \\
\hline 08S06W02ACB1 & 340339 & 913958 & USGS & 68 & 181.03 & 43.49 & 138 & 03/13/2008 \\
\hline 08S07W05DDD1 & 340301 & 914903 & ANRC & 97 & 190 & 30.23 & 160 & 03/14/2008 \\
\hline 09S04W06CBB1 & 335721 & 913252 & NRCS & 110 & 163 & 39 & 124 & 03/12/2008 \\
\hline 09S05W17BCB1 & 335552 & 913820 & USGS & 97 & 171 & 43.17 & 128 & 03/13/2008 \\
\hline 09S05W19CCC1 & 335428 & 913941 & NRCS & 110 & 171 & 40 & 131 & 03/12/2008 \\
\hline 09S06W04BCD1 & 335821 & 914346 & USGS & 62.6 & 181 & 42.58 & 138 & 03/13/2008 \\
\hline 09S06W04BDD1 & 335759 & 914335 & NRCS & 100 & 178 & 46 & 132 & 03/12/2008 \\
\hline 09S06W23CDB1 & 335440 & 914136 & USGS & 70 & 175 & 30.92 & 144 & 03/13/2008 \\
\hline 10S05W06DCC1 & 335155 & 913908 & USGS & 65 & 175 & 32.37 & 143 & 03/13/2008 \\
\hline \multicolumn{9}{|c|}{ Lonoke County } \\
\hline 01N08W03DDA1 & 344411 & 915050 & NRCS & -- & 229 & 137 & 92 & 04/15/2008 \\
\hline 01N09W07DAA1 & 344337 & 920030 & NRCS & -- & 240 & 51 & 189 & 04/15/2008 \\
\hline 01N09W13DAB1 & 344235 & 915517 & USGS & 150 & 226 & 88.43 & 138 & 03/19/2008 \\
\hline 01N09W25BAA1 & 344120 & 915538 & NRCS & -- & 226 & 88 & 138 & 04/15/2008 \\
\hline 01N10W15CDA1 & 344236 & 920415 & NRCS & 100 & 240 & 31 & 209 & 04/15/2008 \\
\hline 01S06W31ABB1 & 343459 & 914131 & ANRC & 120 & 200 & 79.32 & 121 & 03/19/2008 \\
\hline 01S06W32BBB1 & 343501 & 914056 & NRCS & -- & 201 & 78.5 & 123 & 04/15/2008 \\
\hline 01S07W12ABA1 & 343834 & 914230 & USGS & 140 & 207 & 71.77 & 135 & 03/19/2008 \\
\hline 01S08W24CDD1 & 343606 & 914912 & USGS & 127 & 210 & 83.34 & 127 & 03/19/2008 \\
\hline 01S09W36CCC1* & 343435 & 915619 & NRCS & 95 & 220 & 61.91 & 158 & 03/18/2008 \\
\hline 01S09W36CCC1* & 343435 & 915619 & NRCS & 95 & 220 & 64 & 156 & 04/15/2008 \\
\hline 01S10W01ACB1 & 343927 & 920215 & USGS & -- & 236 & 44.63 & 191 & 03/18/2008 \\
\hline 02N07W07DAA1 & 344845 & 914707 & NRCS & -- & 232 & 134 & 98 & 04/15/2008 \\
\hline
\end{tabular}


Appendix 1. Information pertaining to water levels measured in wells completed in the Mississippi River Valley alluvial aquifer in eastern Arkansas, spring 2008.-Continued

[USGS, U.S. Geological Survey; NRCS, Natural Resources Conservation Service, ANRC, Arkansas Natural Resources Commission; --, no data; NGVD of 1929, National Geodetic Vertical Datum of 1929; Horizontal coordinate information is referenced to the North American Datum of 1983 (NAD 83 ); *, control wells for duplicate measurements by USGS and NRCS for quality assurance]

\begin{tabular}{|c|c|c|c|c|c|c|c|c|}
\hline Station nme & $\begin{array}{c}\text { Latitude } \\
\text { (degrees, } \\
\text { minutes, } \\
\text { seconds) }\end{array}$ & $\begin{array}{c}\text { Longitude } \\
\text { (degrees, } \\
\text { minutes, } \\
\text { seconds) }\end{array}$ & $\begin{array}{l}\text { Source } \\
\text { of data }\end{array}$ & $\begin{array}{c}\text { Depth of } \\
\text { well } \\
\text { (feet) }\end{array}$ & $\begin{array}{l}\text { Land-surface } \\
\text { datum altitude } \\
\text { (feet above } \\
\text { NGVD of } \\
\text { 1929) }\end{array}$ & $\begin{array}{l}\text { Depth to } \\
\text { water } \\
\text { (feet below } \\
\text { land-surface } \\
\text { datum) }\end{array}$ & $\begin{array}{c}\text { Water-leve } \\
\text { altitude } \\
\text { (feet above } \\
\text { NGVD of } \\
\text { 1929) }\end{array}$ & $\begin{array}{c}\text { Date of } \\
\text { measure- } \\
\text { ment }\end{array}$ \\
\hline \multicolumn{9}{|c|}{ Lonoke County-Continued } \\
\hline 02N07W16BAB1 & 344815 & 914540 & USGS & 184 & 240 & 135.79 & 104 & 03/19/2008 \\
\hline 02N08W16ABC1 & 344806 & 915114 & USGS & 170 & 230 & 124.87 & 105 & 03/19/2008 \\
\hline 02N08W23CAB1 & 344659 & 915118 & NRCS & -- & 229 & 129 & 100 & 04/15/2008 \\
\hline 02N09W02BDB1 & 344955 & 915841 & ANRC & 140 & 251 & 126.74 & 124 & 03/19/2008 \\
\hline 02N10W15ACC1 & 344807 & 920353 & NRCS & 135 & 241 & 32 & 209 & 04/15/2008 \\
\hline 02S07W05CDC1 & 343326 & 914715 & NRCS & -- & 205 & 70 & 135 & 04/15/2008 \\
\hline 02S07W10CCB1 & 343246 & 914525 & USGS & -- & 201 & 63.34 & 138 & 03/19/2008 \\
\hline 02S08W13BBB1 & 343232 & 914935 & USGS & -- & 200 & 57.67 & 142 & 03/19/2008 \\
\hline 02S08W34DBB1 & 343003 & 915150 & USGS & -- & 214 & 63.76 & 150 & 03/19/2008 \\
\hline 02S09W22AAA1 & 343153 & 915728 & NRCS & -- & 226 & 63 & 163 & 04/15/2008 \\
\hline 02S09W26DC1 & 343019 & 915643 & NRCS & 100 & 216 & 53 & 163 & 04/15/2008 \\
\hline 02S09W30CDD1 & 343014 & 920116 & USGS & 80 & 226 & 38.88 & 187 & 03/18/2008 \\
\hline 03N07W08BDB1 & 345407 & 914638 & USGS & 125 & 250 & 98.31 & 152 & 02/07/2008 \\
\hline 03N07W15DBC2 & 345253 & 914417 & USGS & 144.5 & 227 & 83.53 & 143 & 03/19/2008 \\
\hline 03N07W29ADA1 & 345129 & 914558 & USGS & 120 & 234 & 92.27 & 142 & 02/07/2008 \\
\hline 03N07W29CDD1 & 345057 & 914632 & NRCS & 157 & 232 & 98 & 134 & $04 / 15 / 2008$ \\
\hline 03N07W35CDC2 & 344957 & 914332 & USGS & -- & 232 & 117.23 & 115 & 03/19/2008 \\
\hline 03N08W03BAA1 & 345519 & 915054 & USGS & 162 & 260 & 95.69 & 164 & 02/07/2008 \\
\hline 03N08W03CCC1 & 345430 & 915123 & USGS & 162 & 260 & 105.1 & 155 & 02/07/2008 \\
\hline 03N08W05CCC1 & 345429 & 915323 & USGS & 130 & 257 & 81.03 & 176 & 02/07/2008 \\
\hline 03N08W08ABA1 & 345427 & 915248 & USGS & 150 & 258 & 95.51 & 162 & 02/07/2008 \\
\hline 03N08W10ACB1 & 345415 & 915053 & USGS & 150 & 250 & 92.62 & 157 & 02/07/2008 \\
\hline 03N08W10ADD1 & 345401 & 915023 & USGS & 165 & 250 & 95.05 & 155 & 02/07/2008 \\
\hline 03N08W11ABD1 & 345419 & 914936 & USGS & 160 & 260 & 106.11 & 154 & 02/07/2008 \\
\hline 03N08W11ACA1 & 345413 & 914934 & USGS & 144 & 256 & 104.23 & 152 & 02/07/2008 \\
\hline 03N08W21BCC1 & 345220 & 915220 & USGS & 155 & 247 & 82.79 & 164 & 03/19/2008 \\
\hline 03N08W26CDC1 & 345100 & 915007 & NRCS & 150 & 235 & 111 & 124 & 04/15/2008 \\
\hline 03N08W29BBB1 & 345147 & 915333 & USGS & 152.2 & 249 & 113.34 & 136 & 02/07/2008 \\
\hline 03N08W29BCC1 & 345125 & 915333 & USGS & 150 & 250 & 133.08 & 117 & 02/07/2008 \\
\hline 03N08W32ABB1 & 345057 & 915257 & USGS & 154 & 250 & 120.61 & 129 & 02/07/2008 \\
\hline 03N08W32ABB2 & 345057 & 915259 & USGS & 154 & 250 & 119.68 & 130 & 03/19/2008 \\
\hline 03N08W34ADD1 & 345035 & 915028 & USGS & 130 & 240 & 121.54 & 118 & $11 / 04 / 2008$ \\
\hline 04N08W05ACA1 & 350020 & 915247 & USGS & 138 & 238 & 46.96 & 191 & 02/08/2008 \\
\hline 04N08W10BDD1 & 345917 & 915055 & USGS & 130 & 218 & 30.47 & 188 & 02/08/2008 \\
\hline 04N08W15BCB2 & 345833 & 915121 & USGS & 104 & 225 & 29.2 & 196 & 03/19/2008 \\
\hline
\end{tabular}


Appendix 1. Information pertaining to water levels measured in wells completed in the Mississippi River Valley alluvial aquifer in eastern Arkansas, spring 2008._-Continued

[USGS, U.S. Geological Survey; NRCS, Natural Resources Conservation Service, ANRC, Arkansas Natural Resources Commission; --, no data; NGVD of 1929, National Geodetic Vertical Datum of 1929; Horizontal coordinate information is referenced to the North American Datum of 1983 (NAD 83 ); *, control wells for duplicate measurements by USGS and NRCS for quality assurance]

\begin{tabular}{|c|c|c|c|c|c|c|c|c|}
\hline Station nme & $\begin{array}{c}\text { Latitude } \\
\text { (degrees, } \\
\text { minutes, } \\
\text { seconds) }\end{array}$ & $\begin{array}{c}\text { Longitude } \\
\text { (degrees, } \\
\text { minutes, } \\
\text { seconds) }\end{array}$ & $\begin{array}{l}\text { Source } \\
\text { of data }\end{array}$ & $\begin{array}{c}\text { Depth of } \\
\text { well } \\
\text { (feet) }\end{array}$ & $\begin{array}{l}\text { Land-surface } \\
\text { datum altitude } \\
\text { (feet above } \\
\text { NGVD of } \\
\text { 1929) }\end{array}$ & $\begin{array}{c}\text { Depth to } \\
\text { water } \\
\text { (feet below } \\
\text { land-surface } \\
\text { datum) }\end{array}$ & $\begin{array}{c}\text { Water-leve } \\
\text { altitude } \\
\text { (feet above } \\
\text { NGVD of } \\
\text { 1929) }\end{array}$ & $\begin{array}{c}\text { Date of } \\
\text { measure- } \\
\text { ment }\end{array}$ \\
\hline \multicolumn{9}{|c|}{ Lonoke County-Continued } \\
\hline 04N08W16DCC1 & 345757 & 915154 & USGS & 155 & 225 & 47.88 & 177 & 02/07/2008 \\
\hline 04N08W26AAD1 & 345652 & 914917 & USGS & 130 & 246 & 71.18 & 175 & 02/08/2008 \\
\hline 04N08W28CAC1 & 345620 & 915216 & ANRC & 140.5 & 235 & 55.9 & 179 & 02/07/2008 \\
\hline 04N08W28CAD1 & 345626 & 915204 & USGS & 115 & 249 & 71.23 & 178 & 02/07/2008 \\
\hline 04N08W28CCC1 & 345615 & 915225 & USGS & 137 & 240 & 61.43 & 179 & 02/07/2008 \\
\hline 04N08W33ABD1 & 345559 & 915141 & USGS & 138 & 258 & 85.22 & 173 & 05/01/2008 \\
\hline 04N08W33ABD2 & 345558 & 915141 & USGS & 137 & 258 & 86.73 & 171 & 05/01/2008 \\
\hline 04N08W33ACD1 & 345547 & 915141 & USGS & 152 & 256 & 79.16 & 177 & 05/01/2008 \\
\hline 04N08W33ADB1 & 345553 & 915125 & USGS & 173 & 257 & 94.55 & 162 & 05/01/2008 \\
\hline 04N08W33ADD1 & 345548 & 915125 & USGS & 180 & 265 & 97 & 168 & 05/01/2008 \\
\hline 04N08W33ADD2 & 345546 & 915125 & USGS & 190 & 266 & 96.67 & 169 & 05/01/2008 \\
\hline 04N08W36DBB1 & 345541 & 914914 & USGS & 130 & 259 & 93.08 & 166 & 02/08/2008 \\
\hline \multicolumn{9}{|c|}{ Mississippi County } \\
\hline 10N08E21ABA1 & 352852 & 901415 & NRCS & 110 & 224 & 25 & 199 & 04/17/2008 \\
\hline 10N08E21BDC1 & 352830 & 901407 & NRCS & 100 & 224 & 25.5 & 199 & 04/17/2008 \\
\hline 10N08E22ABA2 & 352851 & 901312 & USGS & 100 & 224 & 25.53 & 198 & 04/07/2008 \\
\hline 10N09E08ACC1 & 352949 & 900926 & ANRC & 110 & 230 & 17.78 & 212 & 04/07/2008 \\
\hline 11N09E34BBB1 & 353218 & 900715 & USGS & 94 & 235 & 19.12 & 216 & 04/07/2008 \\
\hline 11N10E09BCB1 & 353530 & 900202 & NRCS & 110 & 236 & 16 & 220 & 04/17/2008 \\
\hline 12N08E08BCB1 & 354047 & 901559 & USGS & 120 & 225 & 6.26 & 219 & 04/07/2008 \\
\hline 12N08E28DDB1 & 353707 & 901406 & NRCS & 120 & 225 & 15.5 & 210 & 04/17/2008 \\
\hline 12N09E12ABC1 & 354054 & 900449 & NRCS & 120 & 232 & 9 & 223 & 04/25/2008 \\
\hline 12N10E04CAA1 & 354124 & 900136 & NRCS & 120 & 235 & 8 & 227 & 04/17/2008 \\
\hline 12N10E07BCD1 & 354036 & 900404 & NRCS & 110 & 234 & 17 & 217 & $04 / 25 / 2008$ \\
\hline 12N10E21DBA1 & 353842 & 900122 & NRCS & 110 & 236 & 16.5 & 220 & 04/17/2008 \\
\hline 13N08E24ABB1 & 354428 & 901112 & NRCS & 120 & 230 & 7.5 & 223 & 04/17/2008 \\
\hline 13N09E30CCD1 & 354248 & 901029 & USGS & -- & 230 & 7.08 & 223 & 04/07/2008 \\
\hline 13N10E34DBB1 & 354218 & 900024 & ANRC & 98 & 235 & 10.8 & 224 & 04/07/2008 \\
\hline 14N08E12DAB1 & 355104 & 901052 & USGS & -- & 235 & 2.75 & 232 & 04/07/2008 \\
\hline 14N08E20DAA1 & 354921 & 901458 & NRCS & 110 & 225 & 2.5 & 223 & 04/25/2008 \\
\hline $14 \mathrm{~N} 08 \mathrm{E} 26 \mathrm{CC} 1$ & 354803 & 901235 & NRCS & 100 & 230 & 3.5 & 227 & 04/25/2008 \\
\hline $14 \mathrm{~N} 10 \mathrm{E} 18 \mathrm{ABC} 1$ & 355022 & 900345 & USGS & 101 & 236 & 8.11 & 228 & 04/07/2008 \\
\hline 14N11E03BCB1 & 355158 & 895433 & USGS & 128 & 247 & 3.52 & 243 & 04/07/2008 \\
\hline 14N11E17CCB1 & 354955 & 895639 & NRCS & 120 & 240 & 3 & 237 & 04/25/2008 \\
\hline
\end{tabular}


Appendix 1. Information pertaining to water levels measured in wells completed in the Mississippi River Valley alluvial aquifer in eastern Arkansas, spring 2008.-Continued

[USGS, U.S. Geological Survey; NRCS, Natural Resources Conservation Service, ANRC, Arkansas Natural Resources Commission; --, no data; NGVD of 1929, National Geodetic Vertical Datum of 1929; Horizontal coordinate information is referenced to the North American Datum of 1983 (NAD 83 ); *, control wells for duplicate measurements by USGS and NRCS for quality assurance]

\begin{tabular}{|c|c|c|c|c|c|c|c|c|}
\hline Station nme & $\begin{array}{l}\text { Latitude } \\
\text { (degrees, } \\
\text { minutes, } \\
\text { seconds) }\end{array}$ & $\begin{array}{l}\text { Longitude } \\
\text { (degrees, } \\
\text { minutes, } \\
\text { seconds) }\end{array}$ & $\begin{array}{l}\text { Source } \\
\text { of data }\end{array}$ & $\begin{array}{c}\text { Depth of } \\
\text { well } \\
\text { (feet) }\end{array}$ & $\begin{array}{l}\text { Land-surface } \\
\text { datum altitude } \\
\text { (feet above } \\
\text { NGVD of } \\
\text { 1929) }\end{array}$ & $\begin{array}{c}\text { Depth to } \\
\text { water } \\
\text { (feet below } \\
\text { land-surface } \\
\text { datum) }\end{array}$ & $\begin{array}{l}\text { Water-level } \\
\text { altitude } \\
\text { (feet above } \\
\text { NGVD of } \\
\text { 1929) }\end{array}$ & $\begin{array}{l}\text { Date of } \\
\text { measure- } \\
\text { ment }\end{array}$ \\
\hline \multicolumn{9}{|c|}{ Mississippi County-Continued } \\
\hline 14N11E33CAA1 & 354727 & 895508 & NRCS & 120 & 240 & 10 & 230 & $03 / 25 / 2008$ \\
\hline 15N08E08DBC2 & 355605 & 901526 & USGS & 120 & 236 & 10.53 & 225 & 04/07/2008 \\
\hline $15 \mathrm{~N} 10 \mathrm{E} 21 \mathrm{ABC} 1$ & 355447 & 900135 & NRCS & 120 & 240 & 7.5 & 233 & $03 / 25 / 2008$ \\
\hline 15N12E01BCD1 & 355704 & 894601 & NRCS & 100 & 258 & 9 & 249 & $03 / 25 / 2008$ \\
\hline 16N10E28BBD1* & 355906 & 900156 & NRCS & 120 & 238 & 5.5 & 233 & $03 / 25 / 2008$ \\
\hline 16N10E28BBD1* & 355906 & 900156 & NRCS & 120 & 238 & 5.33 & 233 & $04 / 07 / 2008$ \\
\hline 16N11E23ADA1 & 355947 & 895231 & USGS & -- & 255 & 9.2 & 246 & 04/07/2008 \\
\hline \multicolumn{9}{|c|}{ Monroe County } \\
\hline 01N01W21CDC2 & 344037 & 910707 & USGS & 150 & 181 & 39.68 & 141 & $03 / 27 / 2008$ \\
\hline 01N02W12CBC1 & 344242 & 911032 & USGS & 110 & 182 & 40.9 & 141 & $03 / 27 / 2008$ \\
\hline 01N03W23BAC1 & 344124 & 911743 & NRCS & 100 & 170 & 14.3 & 156 & $03 / 20 / 2008$ \\
\hline 01N03W24BBB1 & 344135 & 911651 & USGS & 125 & 185 & 28.23 & 157 & 03/27/2008 \\
\hline 01N04W33BBB2 & 343960 & 912649 & USGS & -- & 218 & 97.56 & 120 & $03 / 27 / 2008$ \\
\hline 01S01W13CDD1 & 343611 & 910341 & USGS & 135 & 178 & 22.52 & 155 & $03 / 27 / 2008$ \\
\hline 01S01W16DB & 343615 & 910632 & NRCS & 100 & 175 & 20 & 155 & $03 / 27 / 2008$ \\
\hline 01S01W18DCD1 & 343618 & 910849 & ANRC & 110 & 178 & 24.52 & 153 & $03 / 27 / 2008$ \\
\hline 01S02W20BBB1* & 343613 & 911456 & NRCS & 100 & 170 & 12 & 158 & 03/27/2008 \\
\hline 01S02W20BBB1* & 343613 & 911456 & NRCS & 100 & 170 & 12.22 & 158 & $03 / 27 / 2008$ \\
\hline 01S03W20BBA1 & 343538 & 912118 & USGS & 140 & 210 & 75.03 & 135 & $03 / 27 / 2008$ \\
\hline 01S04W01BAB1 & 343906 & 912317 & USGS & 160 & 210 & 76.88 & 133 & $03 / 27 / 2008$ \\
\hline 02N01W19ADD1 & 344624 & 910814 & NRCS & 80 & 188 & 54 & 134 & $03 / 27 / 2008$ \\
\hline 02N01W19BBA1 & 344645 & 910912 & USGS & 75 & 191 & 54.78 & 136 & $03 / 27 / 2008$ \\
\hline 02N03W35BCA1 & 344455 & 911745 & NRCS & 100 & 188 & 35 & 153 & 03/20/2008 \\
\hline 02S01W01BCD1 & 343305 & 910408 & NRCS & 100 & 176 & 22 & 154 & 03/27/2008 \\
\hline 02S02W01BCA1 & 343322 & 911031 & USGS & -- & 171 & 13.36 & 158 & $03 / 27 / 2008$ \\
\hline 02S02W11DAC1 & 343209 & 911101 & USGS & 110 & 164 & 11.04 & 153 & $03 / 27 / 2008$ \\
\hline 03N01W20ABA1 & 345201 & 910723 & USGS & -- & 189 & 48.61 & 140 & $03 / 27 / 2008$ \\
\hline 03N02W31ADC1 & 344958 & 911447 & USGS & 95 & 190 & 39.15 & 151 & 03/27/2008 \\
\hline 03N03W36AAA1 & 345027 & 911547 & USGS & 120 & 176 & 23.06 & 153 & $03 / 27 / 2008$ \\
\hline 04N02W01BCC1 & 345929 & 911004 & NRCS & 100 & 175 & 39 & 136 & $03 / 20 / 2008$ \\
\hline 04N02W05BBB1 & 345957 & 911311 & NRCS & 100 & 188 & 16 & 172 & $03 / 20 / 2008$ \\
\hline 04N02W27CDD3 & 345540 & 911150 & USGS & 181 & 200 & 45.68 & 154 & $03 / 27 / 2008$ \\
\hline 04N02W28DDD3 & 345535 & 911221 & USGS & 137 & 192 & 32.81 & 159 & $03 / 27 / 2008$ \\
\hline 04N02W30BBB1 & 345628 & 911525 & USGS & 119 & 185.16 & 13.8 & 171 & $03 / 27 / 2008$ \\
\hline
\end{tabular}


Appendix 1. Information pertaining to water levels measured in wells completed in the Mississippi River Valley alluvial aquifer in eastern Arkansas, spring 2008._-Continued

[USGS, U.S. Geological Survey; NRCS, Natural Resources Conservation Service, ANRC, Arkansas Natural Resources Commission; --, no data; NGVD of 1929, National Geodetic Vertical Datum of 1929; Horizontal coordinate information is referenced to the North American Datum of 1983 (NAD 83 ); *, control wells for duplicate measurements by USGS and NRCS for quality assurance]

\begin{tabular}{|c|c|c|c|c|c|c|c|c|}
\hline Station nme & $\begin{array}{c}\text { Latitude } \\
\text { (degrees, } \\
\text { minutes, } \\
\text { seconds) }\end{array}$ & $\begin{array}{l}\text { Longitude } \\
\text { (degrees, } \\
\text { minutes, } \\
\text { seconds) }\end{array}$ & $\begin{array}{l}\text { Source } \\
\text { of data }\end{array}$ & $\begin{array}{c}\text { Depth of } \\
\text { well } \\
\text { (feet) }\end{array}$ & $\begin{array}{l}\text { Land-surface } \\
\text { datum altitude } \\
\text { (feet above } \\
\text { NGVD of } \\
\text { 1929) }\end{array}$ & $\begin{array}{l}\text { Depth to } \\
\text { water } \\
\text { (feet below } \\
\text { land-surface } \\
\text { datum) }\end{array}$ & $\begin{array}{c}\text { Water-leve } \\
\text { altitude } \\
\text { (feet above } \\
\text { NGVD of } \\
\text { 1929) }\end{array}$ & $\begin{array}{c}\text { Date of } \\
\text { measure- } \\
\text { ment }\end{array}$ \\
\hline \multicolumn{9}{|c|}{ Phillips County } \\
\hline 01S01E20DDB1 & 343529 & 910058 & NRCS & 114 & 185 & 27 & 158 & 04/14/2008 \\
\hline 01S02E09CBB1* & 343719 & 905434 & NRCS & 110 & 185 & 12.8 & 172 & 03/24/2008 \\
\hline 01S02E09CBB1* & 343719 & 905434 & NRCS & 110 & 185 & 15.44 & 170 & 04/01/2008 \\
\hline 01S03E02ADD1 & 343814 & 904511 & NRCS & 120 & 200 & 17 & 183 & $03 / 24 / 2008$ \\
\hline 01S03E10ABB1 & 343741 & 904634 & NRCS & 120 & 205 & 19 & 186 & 03/24/2008 \\
\hline 01S04E05DCD1* & 343802 & 904151 & NRCS & 120 & 230 & 49 & 181 & $03 / 24 / 2008$ \\
\hline 01S04E05DCD1* & 343802 & 904151 & NRCS & 120 & 230 & 50.23 & 180 & 04/01/2008 \\
\hline 02S01E28CCB1 & 342916 & 910058 & USGS & 108 & 174 & 18.63 & 155 & 03/31/2008 \\
\hline 02S02E29DDD1 & 342901 & 905444 & NRCS & 125 & 180 & 24 & 156 & 04/14/2008 \\
\hline 02S02E33ACC1 & 342824 & 905412 & NRCS & 120 & 177 & 24 & 153 & 04/14/2008 \\
\hline 02S03E15ACD1 & 343110 & 904621 & USGS & 112 & 174 & 8.59 & 165 & 05/12/2008 \\
\hline 02S03E34BCD1 & 342828 & 904653 & NRCS & 120 & 165 & 20.4 & 145 & 03/25/2008 \\
\hline 02S04E27AAC1* & 342932 & 904001 & NRCS & 175 & 179 & 7 & 172 & 03/25/2008 \\
\hline 02S04E27AAC1* & 342932 & 904001 & USGS & 175 & 179 & 9.74 & 169 & 03/31/2008 \\
\hline 03S02E35DDA1 & 342256 & 905130 & USGS & 50 & 163 & 22.44 & 141 & 03/31/2008 \\
\hline 03S03E04DAA1 & 342735 & 904710 & USGS & 36 & 171 & 20.37 & 151 & 03/31/2008 \\
\hline 03S04E02CAA1* & 342732 & 903918 & NRCS & 120 & 176 & 15.5 & 161 & 03/25/2008 \\
\hline 03S04E02CAA1* & 342732 & 903918 & USGS & 120 & 176 & 17.48 & 159 & $03 / 31 / 2008$ \\
\hline 04S01E01AAD1 & 342238 & 905700 & NRCS & 120 & 156 & 18 & 138 & 03/25/2008 \\
\hline 04S01E14CDD1 & 342014 & 905837 & NRCS & 120 & 155 & 16 & 139 & 03/25/2008 \\
\hline 04S01E23CCA1 & 341931 & 905853 & USGS & -- & 156 & 14.14 & 142 & 03/31/2008 \\
\hline 04S01E29CDC1 & 341844 & 910148 & NRCS & 120 & 150 & 7 & 143 & 03/25/2008 \\
\hline 04S02E01DBB1 & 342220 & 905053 & NRCS & -- & 163 & 16 & 147 & 03/25/2008 \\
\hline 05S02E18BDA1 & 341535 & 905628 & USGS & 130 & 156 & 23.53 & 132 & $03 / 31 / 2008$ \\
\hline \multicolumn{9}{|c|}{ Poinsett County } \\
\hline 10N01E02AAA & 353205 & 905654 & NRCS & 140 & 235 & 101 & 134 & 04/07/2008 \\
\hline $10 \mathrm{~N} 01 \mathrm{E} 14 \mathrm{CC} 1$ & 352910 & 905814 & ANRC & 150 & 231 & 94.91 & 136 & 04/08/2008 \\
\hline 10N01E16CCB1 & 352922 & 910005 & USGS & 120 & 225 & 77.67 & 147 & 04/08/2008 \\
\hline 10N01E32CBB1 & 352657 & 910053 & NRCS & 120 & 222 & 76.5 & 146 & $03 / 26 / 2008$ \\
\hline 10N01E33ACB1 & 352746 & 905931 & NRCS & 153 & 220 & 81 & 139 & 03/26/2008 \\
\hline 10N02E13BCC1 & 352949 & 905026 & USGS & 167 & 237 & 105.24 & 132 & 04/08/2008 \\
\hline 10N02E15CAA1 & 352940 & 905209 & NRCS & 160 & 237 & 108 & 129 & 03/26/2008 \\
\hline 10N02E20BAB1 & 352906 & 905418 & NRCS & 155 & 237 & 106 & 131 & 03/26/2008 \\
\hline 10N03E13BCB1 & 352958 & 904352 & NRCS & 155 & 275 & 140 & 135 & 03/26/2008 \\
\hline 10N03E14DAB1 & 352947 & 904405 & USGS & -- & 263 & 119.6 & 143 & 04/08/2008 \\
\hline
\end{tabular}


Appendix 1. Information pertaining to water levels measured in wells completed in the Mississippi River Valley alluvial aquifer in eastern Arkansas, spring 2008.-Continued

[USGS, U.S. Geological Survey; NRCS, Natural Resources Conservation Service, ANRC, Arkansas Natural Resources Commission; --, no data; NGVD of 1929, National Geodetic Vertical Datum of 1929; Horizontal coordinate information is referenced to the North American Datum of 1983 (NAD 83 ); *, control wells for duplicate measurements by USGS and NRCS for quality assurance]

\begin{tabular}{|c|c|c|c|c|c|c|c|c|}
\hline Station nme & $\begin{array}{c}\text { Latitude } \\
\text { (degrees, } \\
\text { minutes, } \\
\text { seconds) }\end{array}$ & $\begin{array}{c}\text { Longitude } \\
\text { (degrees, } \\
\text { minutes, } \\
\text { seconds) }\end{array}$ & $\begin{array}{l}\text { Source } \\
\text { of data }\end{array}$ & $\begin{array}{c}\text { Depth of } \\
\text { well } \\
\text { (feet) }\end{array}$ & $\begin{array}{l}\text { Land-surface } \\
\text { datum altitude } \\
\text { (feet above } \\
\text { NGVD of } \\
\text { 1929) }\end{array}$ & $\begin{array}{l}\text { Depth to } \\
\text { water } \\
\text { (feet below } \\
\text { land-surface } \\
\text { datum) }\end{array}$ & $\begin{array}{c}\text { Water-leve } \\
\text { altitude } \\
\text { (feet above } \\
\text { NGVD of } \\
\text { 1929) }\end{array}$ & $\begin{array}{c}\text { Date of } \\
\text { measure- } \\
\text { ment }\end{array}$ \\
\hline \multicolumn{9}{|c|}{ Poinsett County-Continued } \\
\hline 10N03E19BCB1 & 352905 & 904907 & NRCS & -- & 239 & 101 & 138 & 03/26/2008 \\
\hline 10N03E26BBD1 & 352816 & 904449 & NRCS & 140 & 257 & 115 & 142 & 04/07/2008 \\
\hline 10N03E35CDD1 & 352656 & 904436 & USGS & -- & 275 & 125.48 & 150 & 04/08/2008 \\
\hline 10N04E35BBA1 & 352745 & 903831 & NRCS & 100 & 212 & 19.5 & 193 & 03/24/2008 \\
\hline 10N05E15BDD1 & 352937 & 903253 & USGS & -- & 207 & 12.71 & 194 & 04/08/2008 \\
\hline 10N07E22AAC1 & 352847 & 901935 & USGS & -- & 215 & 28.86 & 186 & 04/08/2008 \\
\hline 10N07E28CBB1 & 352733 & 902128 & NRCS & 105 & 217 & 31 & 186 & 03/24/2008 \\
\hline 11N01E17DDC1 & 353437 & 910015 & NRCS & 100 & 232 & 81 & 151 & 04/07/2008 \\
\hline 11N01E17DDD1 & 353437 & 910013 & USGS & 100 & 230 & 80.89 & 149 & 04/08/2008 \\
\hline 11N01E26AA1 & 353340 & 905653 & ANRC & 140 & 236 & 96.63 & 139 & 04/08/2008 \\
\hline 11N01E34AAA & 353256 & 905759 & NRCS & 100 & 229 & 90.5 & 139 & 04/07/2008 \\
\hline 11N02E26AAB1 & 353350 & 905034 & USGS & 158 & 241 & 110.11 & 131 & 04/08/2008 \\
\hline 11N02E30BBB1 & 353352 & 905540 & NRCS & 140 & 239 & 105 & 134 & 04/07/2008 \\
\hline 11N02E34CBA1 & 353238 & 905222 & NRCS & 130 & 240 & 110 & 130 & 04/07/2008 \\
\hline 11N03E10DDA1 & 353546 & 904457 & USGS & 145 & 243 & 106.11 & 137 & 04/08/2008 \\
\hline 11N03E17AAB1 & 353535 & 904714 & NRCS & -- & 242 & 107 & 135 & 04/07/2008 \\
\hline 11N03E18BAB1 & 353538 & 904852 & ANRC & 157 & 243 & 106.53 & 136 & 04/08/2008 \\
\hline 11N04E13DDA1 & 353450 & 903631 & NRCS & 112 & 211 & 18 & 193 & 03/26/2008 \\
\hline 11N04E36ABA1 & 353251 & 903654 & NRCS & 100 & 211 & 16.5 & 195 & 03/24/2008 \\
\hline 11N05E26BDB1 & 353318 & 903213 & NRCS & -- & 213 & 11 & 202 & 03/24/2008 \\
\hline 11N07E18CAB1 & 353435 & 902320 & USGS & 100 & 217 & 14.04 & 203 & 04/08/2008 \\
\hline 11N07E28CBB1 & 353252 & 902120 & NRCS & -- & 217 & 25 & 192 & $03 / 24 / 2008$ \\
\hline 12N01E07CDA1 & 354054 & 910141 & USGS & 120 & 236 & 55.14 & 181 & 04/08/2008 \\
\hline 12N01E22DAB1 & 353922 & 905809 & NRCS & 115 & 235 & 76.5 & 159 & 04/07/2008 \\
\hline 12N02E25DCC1 & 353820 & 904944 & NRCS & 145 & 245 & 115 & 130 & 04/07/2008 \\
\hline 12N02E34CCC1 & 353724 & 905230 & NRCS & 180 & 245 & 115.5 & 130 & 04/07/2008 \\
\hline 12N03E01CBD1 & 354154 & 904329 & NRCS & 190 & 250 & 96 & 154 & 03/26/2008 \\
\hline 12N03E04DAD1 & 354158 & 904600 & USGS & 120 & 247 & 106.12 & 141 & 04/08/2008 \\
\hline 12N03E35AD1 & 353745 & 904353 & NRCS & 150 & 246 & 106 & 140 & 04/07/2008 \\
\hline 12N03E36ACB1 & 353749 & 904319 & USGS & 120 & 250 & 99.86 & 150 & 04/08/2008 \\
\hline 12N04E08CDA & 354053 & 904112 & NRCS & 100 & 250 & 95 & 155 & 03/26/2008 \\
\hline 12N05E16ABA1 & 354039 & 903333 & NRCS & 140 & 221 & 9.5 & 212 & 03/26/2008 \\
\hline 12N05E34ABA1 & 353805 & 903230 & USGS & 100 & 215 & 7.13 & 208 & 04/08/2008 \\
\hline 12N07E04BAA1 & 354202 & 902060 & USGS & 60 & 223 & 2.42 & 221 & 04/08/2008 \\
\hline 12N07E10CBB1 & 354042 & 902022 & NRCS & 100 & 220 & 10 & 210 & 03/24/2008 \\
\hline
\end{tabular}


Appendix 1. Information pertaining to water levels measured in wells completed in the Mississippi River Valley alluvial aquifer in eastern Arkansas, spring 2008. - Continued

[USGS, U.S. Geological Survey; NRCS, Natural Resources Conservation Service, ANRC, Arkansas Natural Resources Commission; --, no data; NGVD of 1929, National Geodetic Vertical Datum of 1929; Horizontal coordinate information is referenced to the North American Datum of 1983 (NAD 83); *, control wells for duplicate measurements by USGS and NRCS for quality assurance]

\begin{tabular}{|c|c|c|c|c|c|c|c|c|}
\hline Station nme & $\begin{array}{c}\text { Latitude } \\
\text { (degrees, } \\
\text { minutes, } \\
\text { seconds) }\end{array}$ & $\begin{array}{l}\text { Longitude } \\
\text { (degrees, } \\
\text { minutes, } \\
\text { seconds) }\end{array}$ & $\begin{array}{l}\text { Source } \\
\text { of data }\end{array}$ & $\begin{array}{c}\text { Depth of } \\
\text { well } \\
\text { (feet) }\end{array}$ & $\begin{array}{l}\text { Land-surface } \\
\text { datum altitude } \\
\text { (feet above } \\
\text { NGVD of } \\
\text { 1929) }\end{array}$ & $\begin{array}{c}\text { Depth to } \\
\text { water } \\
\text { (feet below } \\
\text { land-surface } \\
\text { datum) }\end{array}$ & $\begin{array}{c}\text { Water-leve } \\
\text { altitude } \\
\text { (feet above } \\
\text { NGVD of } \\
\text { 1929) }\end{array}$ & $\begin{array}{c}\text { Date of } \\
\text { measure- } \\
\text { ment }\end{array}$ \\
\hline \multicolumn{9}{|c|}{ Prairie County } \\
\hline 01N06W05CCB1 & 344353 & 914049 & USGS & 155 & 220 & 119.27 & 101 & 03/26/2008 \\
\hline 01N06W29DDD1 & 344018 & 913951 & USGS & 155 & 235 & 118.13 & 117 & 03/26/2008 \\
\hline 01S04W28BBC1 & 343529 & 912650 & NRCS & 180 & 206 & 100 & 106 & 04/02/2008 \\
\hline 01S04W28BDB1 & 343523 & 912630 & USGS & 112 & 205 & 98 & 107 & $03 / 25 / 2008$ \\
\hline 01S05W14BBC1 & 343722 & 913109 & USGS & 118 & 211 & 109.68 & 101 & 03/25/2008 \\
\hline 01S05W31DDA1 & 343417 & 913432 & USGS & 120 & 206 & 105.57 & 100 & $03 / 26 / 2008$ \\
\hline 02N04W02BCB1 & 344916 & 912419 & USGS & 140 & 188 & 19.88 & 168 & 03/25/2008 \\
\hline 02N04W32CCB1 & 344436 & 912738 & USGS & -- & 221 & 84.84 & 136 & 03/25/2008 \\
\hline 02N05W06BAB1 & 344958 & 913421 & USGS & 145 & 221 & 89.81 & 131 & 03/26/2008 \\
\hline 02N05W13AAB1 & 344805 & 912854 & USGS & 130 & 223 & 51.83 & 171 & 03/25/2008 \\
\hline 02N05W29DDB2 & 344545 & 913309 & USGS & 135 & 228 & 119.78 & 108 & 03/26/2008 \\
\hline 02N06W17ABB1 & 344809 & 913959 & ANRC & 180 & 235 & 125.82 & 109 & 03/26/2008 \\
\hline 02S06W14BBB1 & 343213 & 913729 & USGS & 105 & 201 & 75.76 & 125 & 03/26/2008 \\
\hline 03N04W03AAC1 & 345439 & 912424 & USGS & 106 & 187 & 26.91 & 160 & 03/25/2008 \\
\hline 03N05W03BDD2 & 345444 & 913115 & USGS & 110 & 207 & 65.25 & 142 & 03/26/2008 \\
\hline 03N06W01BCB1 & 345455 & 913601 & USGS & 115 & 216 & 80.72 & 135 & 03/26/2008 \\
\hline 03N06W19BDD1 & 345207 & 914110 & USGS & 105 & 221 & 86.33 & 135 & 03/26/2008 \\
\hline 04N04W07ADC1 & 345850 & 912733 & USGS & 110 & 195 & 23.66 & 171 & 03/25/2008 \\
\hline 04N05W07CDC1 & 345043 & 913441 & USGS & -- & 212 & 79.34 & 133 & 03/25/2008 \\
\hline 04N05W31DDC1 & 345514 & 913406 & USGS & 104 & 206 & 76.25 & 130 & 03/26/2008 \\
\hline 04N06W05CCC1 & 345934 & 914018 & USGS & 100 & 206 & 80.49 & 126 & 03/25/2008 \\
\hline 04N07W03DCB1 & 345942 & 914412 & ANRC & 100 & 255 & 88.73 & 166 & 03/25/2008 \\
\hline 04N07W20DDB1 & 345709 & 914607 & USGS & 160 & 255 & 103.11 & 152 & 02/07/2008 \\
\hline 04N07W28BBA1 & 345701 & 914545 & ANRC & 110 & 258 & 97.67 & 160 & 03/25/2008 \\
\hline 05N05W14DCD1 & 350252 & 913034 & USGS & -- & 205 & 31.74 & 173 & 03/25/2008 \\
\hline \multicolumn{9}{|c|}{ Pulaski County } \\
\hline 01S10W29CC1 & 343538 & 920708 & USGS & 100 & 239 & 14.87 & 224 & 03/18/2008 \\
\hline 02S10W14DC1 & 343205 & 920334 & USGS & 60 & 225 & 23.5 & 202 & 03/18/2008 \\
\hline 02S10W16CCA1 & 343217 & 920549 & USGS & -- & 230.76 & 25.33 & 205 & 03/18/2008 \\
\hline \multicolumn{9}{|c|}{ Randolph County } \\
\hline 18N01E13BAB1 & 361230 & 905551 & NRCS & 100 & 266 & 16 & 250 & 04/08/2008 \\
\hline 18N01E28AAD1 & 361040 & 905820 & NRCS & 120 & 265 & 13.5 & 252 & 04/08/2008 \\
\hline 18N01E34AAC1 & 360943 & 905729 & USGS & -- & 266 & 15.9 & 250 & 04/10/2008 \\
\hline 18N02E03DAD1 & 361336 & 905043 & NRCS & 120 & 280 & 32.5 & 248 & 04/08/2008 \\
\hline 18N02E17CBB1 & 361204 & 905356 & NRCS & -- & 265 & 19 & 246 & 04/08/2008 \\
\hline
\end{tabular}


Appendix 1. Information pertaining to water levels measured in wells completed in the Mississippi River Valley alluvial aquifer in eastern Arkansas, spring 2008.-Continued

[USGS, U.S. Geological Survey; NRCS, Natural Resources Conservation Service, ANRC, Arkansas Natural Resources Commission; --, no data; NGVD of 1929, National Geodetic Vertical Datum of 1929; Horizontal coordinate information is referenced to the North American Datum of 1983 (NAD 83 ); *, control wells for duplicate measurements by USGS and NRCS for quality assurance]

\begin{tabular}{|c|c|c|c|c|c|c|c|c|}
\hline Station nme & $\begin{array}{l}\text { Latitude } \\
\text { (degrees, } \\
\text { minutes, } \\
\text { seconds) }\end{array}$ & $\begin{array}{l}\text { Longitude } \\
\text { (degrees, } \\
\text { minutes, } \\
\text { seconds) }\end{array}$ & $\begin{array}{l}\text { Source } \\
\text { of data }\end{array}$ & $\begin{array}{c}\text { Depth of } \\
\text { well } \\
\text { (feet) }\end{array}$ & $\begin{array}{l}\text { Land-surface } \\
\text { datum altitude } \\
\text { (feet above } \\
\text { NGVD of } \\
\text { 1929) }\end{array}$ & $\begin{array}{c}\text { Depth to } \\
\text { water } \\
\text { (feet below } \\
\text { land-surface } \\
\text { datum) }\end{array}$ & $\begin{array}{l}\text { Water-level } \\
\text { altitude } \\
\text { (feet above } \\
\text { NGVD of } \\
\text { 1929) }\end{array}$ & $\begin{array}{l}\text { Date of } \\
\text { measure- } \\
\text { ment }\end{array}$ \\
\hline \multicolumn{9}{|c|}{ Randolph County-Continued } \\
\hline 18N02E20BDA1 & 361125 & 905332 & NRCS & 110 & 274 & 32.5 & 242 & $04 / 08 / 2008$ \\
\hline 18N02E22DCD1 & 361046 & 905105 & ANRC & 110 & 273 & 39.12 & 234 & 04/10/2008 \\
\hline 18N02E34BCC1 & 360933 & 905150 & NRCS & 100 & 265 & 35.5 & 230 & $04 / 08 / 2008$ \\
\hline 19N02E09DCA1 & 361757 & 905157 & USGS & -- & 267 & -0.5 & 268 & $04 / 10 / 2008$ \\
\hline 20N02E01ADD1 & 362424 & 904811 & ANRC & 65 & 280 & 2.66 & 277 & $04 / 10 / 2008$ \\
\hline 20N02E01ADD2 & 362424 & 904811 & NRCS & 65 & 281 & 3 & 278 & $04 / 10 / 2008$ \\
\hline 20N02E12BAA1 & 362352 & 904848 & NRCS & 60 & 281 & 19 & 262 & 04/09/2008 \\
\hline 20N02E14DAB1 & 362232 & 904930 & NRCS & 100 & 274 & 18 & 256 & $04 / 10 / 2008$ \\
\hline 20N02E21CDD1 & 362117 & 905107 & NRCS & 110 & 270 & 9 & 261 & $04 / 10 / 2008$ \\
\hline 20N03E28BA1 & 362114 & 904538 & ANRC & -- & 276 & 9.43 & 267 & $94 / 10 / 2008$ \\
\hline 20N03E33CCA1 & 361941 & 904552 & NRCS & -- & 287 & 23.5 & 264 & 04/09/2008 \\
\hline \multicolumn{9}{|c|}{ St. Francis County } \\
\hline 04N01E13ADA1 & 345755 & 905638 & USGS & -- & 206 & 61.56 & 144 & $04 / 02 / 2008$ \\
\hline 04N01W28CDD1 & 345535 & 910634 & USGS & -- & 208 & 72.51 & 135 & $94 / 02 / 2008$ \\
\hline 04N02E19BBB1 & 345701 & 905633 & USGS & 72.2 & 209 & 61.77 & 147 & $04 / 02 / 2008$ \\
\hline 04N03E21DAD1 & 345623 & 904655 & USGS & -- & 236 & 60.37 & 176 & $04 / 02 / 2008$ \\
\hline 04N05E22BBB1 & 345651 & 903357 & USGS & -- & 200 & 27.22 & 173 & $04 / 02 / 2008$ \\
\hline 05N01E15BCB1 & 350303 & 905942 & USGS & 94.1 & 209 & 69.66 & 139 & $04 / 02 / 2008$ \\
\hline 05N01E27BBA1 & 350136 & 905929 & USGS & -- & 209 & 69.07 & 140 & 04/02/2008 \\
\hline 05N02E20ADC1 & 350157 & 905437 & USGS & 79 & 211 & 56.07 & 155 & $04 / 02 / 2008$ \\
\hline 05N03E20AAA2 & 350214 & 904801 & USGS & 153.45 & 250 & 106.09 & 144 & $04 / 02 / 2008$ \\
\hline 05N05E19DCA1 & 350128 & 903630 & ANRC & 110 & 203 & 32.66 & 170 & $94 / 02 / 2008$ \\
\hline 05N06E34CAB1 & 350026 & 902657 & USGS & 110 & 200 & 28.38 & 172 & $04 / 02 / 2008$ \\
\hline 06N01E33ACA2 & 350552 & 905942 & USGS & -- & 211 & 69.41 & 142 & $04 / 02 / 2008$ \\
\hline 06N02E13DCA1 & 350813 & 905003 & USGS & -- & 231 & 75.8 & 155 & $04 / 02 / 2008$ \\
\hline 06N02E15BDD1 & 350842 & 905247 & USGS & 75 & 214.64 & 62.23 & 152 & $04 / 02 / 2008$ \\
\hline 06N02E24AAA1 & 350755 & 905002 & USGS & 147 & 232 & 72.94 & 159 & $04 / 02 / 2008$ \\
\hline 06N05E22ACC1 & 350723 & 903252 & USGS & -- & 200 & 42.82 & 157 & $04 / 02 / 2008$ \\
\hline 06N06E20ABB2 & 350747 & 902841 & ANRC & 150 & 200 & 37.3 & 163 & $04 / 02 / 2008$ \\
\hline \multicolumn{9}{|c|}{ White County } \\
\hline 05N07W09AAA1 & 350447 & 914441 & USGS & 29.5 & 205 & 12.62 & 192 & $04 / 16 / 2008$ \\
\hline 05N07W10CCC1 & 350400 & 914436 & USGS & 80 & 203 & 7.79 & 195 & $04 / 16 / 2008$ \\
\hline 06N06W04BAA1 & 351047 & 913910 & ANRC & 70 & 220 & 28.9 & 191 & $94 / 16 / 2008$ \\
\hline 06N06W04BAD1 & 351037 & 913903 & NRCS & -- & 215 & 36.7 & 178 & 03/27/2008 \\
\hline 06N06W13DBB1 & 350918 & 913552 & NRCS & -- & 213 & 45.8 & 167 & $03 / 27 / 2008$ \\
\hline
\end{tabular}


Appendix 1. Information pertaining to water levels measured in wells completed in the Mississippi River Valley alluvial aquifer in eastern Arkansas, spring 2008._-Continued

[USGS, U.S. Geological Survey; NRCS, Natural Resources Conservation Service, ANRC, Arkansas Natural Resources Commission; --, no data; NGVD of 1929, National Geodetic Vertical Datum of 1929; Horizontal coordinate information is referenced to the North American Datum of 1983 (NAD 83 ); *, control wells for duplicate measurements by USGS and NRCS for quality assurance]

\begin{tabular}{|c|c|c|c|c|c|c|c|c|}
\hline Station nme & $\begin{array}{l}\text { Latitude } \\
\text { (degrees, } \\
\text { minutes, } \\
\text { seconds) }\end{array}$ & $\begin{array}{l}\text { Longitude } \\
\text { (degrees, } \\
\text { minutes, } \\
\text { seconds) }\end{array}$ & $\begin{array}{l}\text { Source } \\
\text { of data }\end{array}$ & $\begin{array}{c}\text { Depth of } \\
\text { well } \\
\text { (feet) }\end{array}$ & $\begin{array}{l}\text { Land-surface } \\
\text { datum altitude } \\
\text { (feet above } \\
\text { NGVD of } \\
\text { 1929) }\end{array}$ & $\begin{array}{l}\text { Depth to } \\
\text { water } \\
\text { (feet below } \\
\text { land-surface } \\
\text { datum) }\end{array}$ & $\begin{array}{c}\text { Water-leve } \\
\text { altitude } \\
\text { (feet above } \\
\text { NGVD of } \\
\text { 1929) }\end{array}$ & $\begin{array}{c}\text { Date of } \\
\text { measure- } \\
\text { ment }\end{array}$ \\
\hline \multicolumn{9}{|c|}{ White County-Continued } \\
\hline 06N06W18BBC1 & 350851 & 914152 & USGS & -- & 210 & 12.09 & 198 & 04/16/2008 \\
\hline 06N06W18BCA1 & 350835 & 914150 & NRCS & -- & 210 & 13.8 & 196 & 03/27/2008 \\
\hline 06N06W34AAB1 & 350624 & 913754 & USGS & -- & 213 & 60.78 & 152 & 04/16/2008 \\
\hline 06N07W17DCC1 & 350822 & 914635 & USGS & 90 & 217 & 13.42 & 204 & $04 / 16 / 2008$ \\
\hline 06N08W13ABA1 & 350908 & 914824 & USGS & 60 & 228 & 6.6 & 221 & 04/16/2008 \\
\hline 06N08W26DDB1 & 350640 & 914931 & USGS & 89 & 230 & 13.23 & 217 & $04 / 16 / 2008$ \\
\hline 07N05W01AAA1 & 351553 & 912858 & USGS & -- & 205 & 11.11 & 194 & $04 / 16 / 2008$ \\
\hline 07N05W32BAB1 & 351137 & 913406 & USGS & 80 & 213.7 & 33.17 & 181 & 04/16/2008 \\
\hline 08N04W06CCB1 & 352028 & 912847 & USGS & 74 & 214 & 12.81 & 201 & 04/16/2008 \\
\hline 08N05W32CBC1 & 351616 & 913417 & USGS & -- & 199 & 3.46 & 196 & $04 / 16 / 2008$ \\
\hline \multicolumn{9}{|c|}{ Woodruff County } \\
\hline 04N03W03AB1 & 350021 & 911820 & ANRC & 100 & 185 & 9.63 & 175 & $04 / 11 / 2008$ \\
\hline 05N01W13CDC1 & 350244 & 910331 & NRCS & 135 & 210 & 76.6 & 133 & 04/17/2008 \\
\hline 05N01W31CCC1 & 350106 & 910900 & NRCS & 140 & 210 & 61.2 & 149 & 04/17/2008 \\
\hline 05N02W20DCB1 & 350208 & 911356 & USGS & -- & 192 & 11.87 & 180 & 04/11/2008 \\
\hline 05N03W25DDB1 & 350133 & 911531 & NRCS & 120 & 190 & 10.6 & 179 & $04 / 17 / 2008$ \\
\hline 05N04W12DBA1 & 350427 & 912211 & USGS & 92 & 186 & 2.94 & 183 & $04 / 15 / 2008$ \\
\hline 06N01W06BAB1 & 351048 & 910835 & USGS & -- & 202 & 36.73 & 165 & 04/11/2008 \\
\hline 06N02W19AAA1 & 350802 & 911419 & NRCS & 130 & 225 & 45.9 & 179 & 04/17/2008 \\
\hline 06N03W31BCB1 & 350623 & 912144 & USGS & -- & 185 & 1.5 & 184 & $04 / 15 / 2008$ \\
\hline 06N04W22BDA1 & 350807 & 912428 & NRCS & 120 & 186 & -1 & 187 & $04 / 17 / 2008$ \\
\hline 07N01W04ACB1 & 351541 & 910626 & NRCS & 125 & 225 & 64.2 & 161 & $04 / 17 / 2008$ \\
\hline 07N03W06BAC1 & 351607 & 912109 & NRCS & 100 & 211 & 23.5 & 188 & $03 / 21 / 2008$ \\
\hline 07N03W19AAA1 & 351335 & 912025 & USGS & 100 & 202.59 & 8.39 & 194 & 04/15/2008 \\
\hline 07N03W31BBA1 & 351152 & 912103 & NRCS & 120 & 195 & 7.7 & 187 & 04/17/2008 \\
\hline 08N01W06DDD1 & 352028 & 910747 & USGS & -- & 218 & 46.14 & 172 & 04/11/2008 \\
\hline 08N02W27DDB1 & 351711 & 911107 & NRCS & 100 & 214 & 27.5 & 187 & 04/17/2008 \\
\hline 08N02W31DDD1 & 351611 & 911411 & USGS & 40 & 194.55 & 1.66 & 193 & $04 / 11 / 2008$ \\
\hline 08N03W31AAD1 & 351655 & 912028 & USGS & 110 & 212 & 22.78 & 189 & $04 / 15 / 2008$ \\
\hline 09N03W28ABB1 & 352310 & 911845 & NRCS & 120 & 220 & 17.6 & 202 & 04/17/2008 \\
\hline 09N03W29AAD1 & 352258 & 911921 & USGS & -- & 220 & 18.9 & 201 & $04 / 15 / 2008$ \\
\hline 09N03W32ACA1 & 352205 & 911936 & NRCS & 120 & 217 & 16.9 & 200 & 04/17/2008 \\
\hline
\end{tabular}


Appendix 2. Information pertaining to water levels measured in 2004 and 2008 in the Mississippi River Valley alluvial aquifer in eastern Arkansas.

[USGS, U.S. Geological Survey; NRCS, Natural Resources Conservation Service, ANRC, Arkansas Natural Resources Commission; Horizontal coordinate information is referenced to the North American Datum of 1983 (NAD83); *, control wells for duplicate measurements by USGS and NRCS for quality assurance]

\begin{tabular}{|c|c|c|c|c|c|c|c|}
\hline Station name & $\begin{array}{l}\text { Latitude } \\
\text { (degrees, } \\
\text { minutes, } \\
\text { seconds) }\end{array}$ & $\begin{array}{l}\text { Longitude } \\
\text { (degrees, } \\
\text { minutes, } \\
\text { seconds) }\end{array}$ & $\begin{array}{l}2004 \text { water- } \\
\text { level date }\end{array}$ & $\begin{array}{c}2004 \text { depth } \\
\text { to water } \\
\text { (feet below } \\
\text { land-surface } \\
\text { datum) }\end{array}$ & $\begin{array}{l}2008 \text { water- } \\
\text { level date }\end{array}$ & $\begin{array}{c}2008 \text { depth } \\
\text { to water } \\
\text { (feet below } \\
\text { land-surface } \\
\text { datum) }\end{array}$ & $\begin{array}{c}\text { Water-level } \\
\text { difference } \\
2004 \text { to } 2008 \\
\text { (feet) }\end{array}$ \\
\hline \multicolumn{8}{|c|}{ Arkansas County } \\
\hline 02S04W11DBB1 & 343233 & 912415 & $3 / 09 / 2004$ & 99.78 & $3 / 24 / 2008$ & 100.74 & -1.0 \\
\hline 02S05W15AAB1 & 343213 & 913127 & 3/09/2004 & 107.66 & $3 / 24 / 2008$ & 107.38 & 0.3 \\
\hline 02S05W31BBB1 & 342937 & 913536 & $3 / 09 / 2004$ & 38.18 & $3 / 24 / 2008$ & 41.24 & -3.1 \\
\hline 03S02W27ABB1 & 342448 & 911251 & $3 / 09 / 2004$ & 65.58 & $3 / 25 / 2008$ & 65.92 & -0.3 \\
\hline 03S03W05CCD1 & 342737 & 912132 & $3 / 09 / 2004$ & 97.85 & $3 / 24 / 2008$ & 99.78 & -1.9 \\
\hline 03S03W27BBC1 & 342455 & 911944 & $3 / 04 / 2004$ & 90.83 & $3 / 24 / 2008$ & 93.04 & -2.2 \\
\hline 03S04W02BBB1 & 342831 & 912454 & $3 / 04 / 2004$ & 91.92 & $3 / 24 / 2008$ & 93.22 & -1.3 \\
\hline 03S04W03DCA16 & 342753 & 912515 & $3 / 04 / 2004$ & 100.02 & $5 / 12 / 2008$ & 101.14 & -1.1 \\
\hline 03S05W03CCC1 & 342752 & 913227 & $3 / 08 / 2004$ & 104.87 & $3 / 20 / 2008$ & 104.42 & 0.5 \\
\hline 03S06W35ADD1 & 342411 & 913652 & $3 / 08 / 2004$ & 51.98 & $3 / 21 / 2008$ & 54.81 & -2.8 \\
\hline 04S01W04ACD2 & 342233 & 910733 & $3 / 09 / 2004$ & 5.62 & $3 / 25 / 2008$ & 4.05 & 1.6 \\
\hline 04S01W31DCB1 & 341753 & 910949 & $3 / 09 / 2004$ & 52.04 & $3 / 25 / 2008$ & 52.42 & -0.4 \\
\hline 04S02W11AAA1 & 342209 & 911123 & $3 / 09 / 2004$ & 66.4 & $3 / 25 / 2008$ & 67.81 & -1.4 \\
\hline 04S02W29CCC1 & 341846 & 911539 & $3 / 09 / 2004$ & 81.8 & $3 / 25 / 2008$ & 83.92 & -2.1 \\
\hline 04S03W17ADD1 & 342102 & 912058 & $3 / 04 / 2004$ & 107.08 & $3 / 24 / 2008$ & 109.98 & -2.9 \\
\hline 04S03W32BCB1 & 341820 & 912202 & $3 / 04 / 2004$ & 116.43 & $3 / 20 / 2008$ & 123.19 & -6.8 \\
\hline 04S04W02ABB1 & 342313 & 912424 & $3 / 04 / 2004$ & 108.54 & $3 / 24 / 2008$ & 109.89 & -1.4 \\
\hline 04S04W35ABC1 & 341835 & 912437 & $4 / 09 / 2004$ & 103.5 & $4 / 07 / 2008$ & 106 & -2.5 \\
\hline 04S05W16CDC1 & 342045 & 913321 & $3 / 08 / 2004$ & 70.58 & $3 / 20 / 2008$ & 71.25 & -0.7 \\
\hline 04S05W24DAA1 & 342001 & 912930 & $3 / 08 / 2004$ & 90.49 & $3 / 20 / 2008$ & 90.17 & 0.3 \\
\hline 04S06W15DBB1 & 342122 & 913827 & $3 / 08 / 2004$ & 32.84 & $3 / 21 / 2008$ & 34.88 & -2.0 \\
\hline 05S01W16BAB1 & 341552 & 910729 & 3/09/2004 & 49.61 & $3 / 25 / 2008$ & 51.37 & -1.8 \\
\hline 05S02W16ABD1 & 341552 & 911358 & $3 / 04 / 2004$ & 79.36 & $3 / 20 / 2008$ & 86.13 & -6.8 \\
\hline 05S04W07CCC1 & 341555 & 912932 & $3 / 08 / 2004$ & 75.2 & $3 / 20 / 2008$ & 75.05 & 0.2 \\
\hline 05S04W32BBA1 & 341316 & 912822 & $3 / 08 / 2004$ & 58.03 & $3 / 20 / 2008$ & 58.75 & -0.7 \\
\hline 05S06W02DDD1 & 341724 & 913651 & $3 / 08 / 2004$ & 20.48 & $3 / 21 / 2008$ & 21.8 & -1.3 \\
\hline 05S06W07DDC1 & 341642 & 914130 & $3 / 08 / 2004$ & 2.78 & $3 / 21 / 2008$ & 3.83 & -1.1 \\
\hline 06S02W23DCD1 & 340853 & 911206 & $3 / 04 / 2004$ & 68.17 & $3 / 20 / 2008$ & 70.61 & -2.4 \\
\hline 06S03W10BBA1 & 341136 & 911954 & $3 / 04 / 2004$ & 82.42 & $3 / 20 / 2008$ & 82.25 & 0.2 \\
\hline 06S03W27AAA1 & 340858 & 911913 & $3 / 04 / 2004$ & 66.72 & $3 / 20 / 2008$ & 68.55 & -1.8 \\
\hline 07S02W04BBB1 & 340707 & 911452 & $3 / 04 / 2004$ & 35.38 & $3 / 20 / 2008$ & 50.5 & -15.1 \\
\hline 07S02W17BBA1 & 340530 & 911539 & $3 / 04 / 2004$ & 52.23 & $3 / 20 / 2008$ & 54.05 & -1.8 \\
\hline 07S03W18CCD1 & 340435 & 912316 & $3 / 04 / 2004$ & 43.08 & $3 / 20 / 2008$ & 44.17 & -1.1 \\
\hline 07S03W32BBC1 & 340240 & 912216 & $3 / 04 / 2004$ & 25.5 & $3 / 20 / 2008$ & 26.45 & -1.0 \\
\hline
\end{tabular}


Appendix 2. Information pertaining to water levels measured in 2004 and 2008 in the Mississippi River Valley alluvial aquifer in eastern Arkansas.-Continued

[USGS, U.S. Geological Survey; NRCS, Natural Resources Conservation Service, ANRC, Arkansas Natural Resources Commission; Horizontal coordinate information is referenced to the North American Datum of 1983 (NAD83); *, control wells for duplicate measurements by USGS and NRCS for quality assurance]

\begin{tabular}{|c|c|c|c|c|c|c|c|}
\hline Station name & $\begin{array}{l}\text { Latitude } \\
\text { (degrees, } \\
\text { minutes, } \\
\text { seconds) }\end{array}$ & $\begin{array}{c}\text { Longitude } \\
\text { (degrees, } \\
\text { minutes, } \\
\text { seconds) }\end{array}$ & $\begin{array}{l}2004 \text { water- } \\
\text { level date }\end{array}$ & $\begin{array}{l}2004 \text { depth } \\
\text { to water } \\
\text { (feet below } \\
\text { land-surface } \\
\text { datum) }\end{array}$ & $\begin{array}{l}2008 \text { water- } \\
\text { level date }\end{array}$ & $\begin{array}{l}2008 \text { depth } \\
\text { to water } \\
\text { (feet below } \\
\text { land-surface } \\
\text { datum) }\end{array}$ & $\begin{array}{c}\text { Water-level } \\
\text { difference } \\
2004 \text { to } 2008 \\
\text { (feet) }\end{array}$ \\
\hline \multicolumn{8}{|c|}{ Arkansas County-Continued } \\
\hline 07S04W01DDD1 & 340625 & 912327 & $3 / 04 / 2004$ & 44.52 & $3 / 20 / 2008$ & 48.48 & -4.0 \\
\hline 08S02W08ACA1 & 340041 & 911506 & $3 / 04 / 2004$ & 42.23 & $3 / 20 / 2008$ & 42.67 & -0.4 \\
\hline 08S03WT2299 & 340147 & 912203 & $3 / 04 / 2004$ & 21.62 & $3 / 20 / 2008$ & 22.09 & -0.5 \\
\hline \multicolumn{8}{|c|}{ Ashley County } \\
\hline 15S04W23DBD1 & 332247 & 912852 & $2 / 26 / 2004$ & 32.29 & $3 / 11 / 2008$ & 33.42 & -1.1 \\
\hline 15S04W26DCC1 & 332232 & 912902 & $2 / 26 / 2004$ & 30.97 & $3 / 11 / 2008$ & 32.17 & -1.2 \\
\hline 15S07W21CBA1 & 332316 & 915001 & $2 / 25 / 2004$ & 3.74 & $3 / 10 / 2008$ & 4.52 & -0.8 \\
\hline 16S06W08CAA1 & 331941 & 914438 & $2 / 25 / 2004$ & 77.22 & $3 / 10 / 2008$ & 78.92 & -1.7 \\
\hline 16S06W27BAB1 & 331729 & 914240 & $2 / 25 / 2004$ & 83.2 & $3 / 10 / 2008$ & 84.07 & -0.9 \\
\hline 17S04W03ABB1 & 331528 & 913010 & $2 / 26 / 2004$ & 28.97 & $3 / 11 / 2008$ & 30.77 & -1.8 \\
\hline 17S04W15DDC1 & 331252 & 912954 & $2 / 26 / 2004$ & 26.45 & $3 / 11 / 2008$ & 27.7 & -1.3 \\
\hline 17S04W21ABA1 & 331252 & 913108 & $2 / 26 / 2004$ & 21.37 & $3 / 11 / 2008$ & 22.97 & -1.6 \\
\hline 17S06W01ADD1 & 331518 & 913956 & $2 / 25 / 2004$ & 82.57 & $3 / 10 / 2008$ & 84.03 & -1.5 \\
\hline 17S06W35CAC1 & 331049 & 914136 & $2 / 25 / 2004$ & 78.29 & $3 / 10 / 2008$ & 72.41 & 5.9 \\
\hline 18S04W23DDD1 & 330658 & 912856 & $4 / 30 / 2004$ & 22 & $4 / 15 / 2008$ & 30 & -8.0 \\
\hline 18S05W11CCD1 & 330841 & 913538 & $4 / 30 / 2004$ & 16 & $4 / 15 / 2008$ & 27 & -11.0 \\
\hline 18S05W22DDA1 & 330712 & 913555 & $4 / 30 / 2004$ & 12 & $4 / 15 / 2008$ & 22 & -10.0 \\
\hline 18S08W01AAB1 & 331015 & 915225 & $2 / 25 / 2004$ & 86.33 & $3 / 10 / 2008$ & 84.34 & 2.0 \\
\hline 18S08W28DDD2 & 330625 & 915528 & $2 / 26 / 2004$ & 85.16 & $5 / 13 / 2008$ & 85.11 & 0.1 \\
\hline 19S04W06BAB2 & 330504 & 913329 & $2 / 26 / 2004$ & 24.07 & $3 / 11 / 2008$ & 23.92 & 0.2 \\
\hline 19S04W14BBB1 & 330310 & 912913 & $4 / 30 / 2004$ & 20 & $4 / 15 / 2008$ & 31 & -11.0 \\
\hline 19S05W08ACA1 & 330405 & 913815 & $4 / 30 / 2004$ & 11 & $4 / 15 / 2008$ & 18 & -7.0 \\
\hline 19S05W16ABB1 & 330323 & 913718 & $4 / 30 / 2004$ & 19 & $4 / 15 / 2008$ & 28 & -9.0 \\
\hline 19S05W22DCD1 & 330139 & 913615 & $4 / 30 / 2004$ & 20 & $4 / 15 / 2008$ & 26 & -6.0 \\
\hline 19S06W07BCC1 & 330404 & 914608 & $2 / 25 / 2004$ & 31.43 & $3 / 10 / 2008$ & 32.46 & -1.0 \\
\hline \multicolumn{8}{|c|}{ Chicot County } \\
\hline 13S03W27AAA1 & 333253 & 912310 & $4 / 28 / 2004$ & 43 & $3 / 24 / 2008$ & 48 & -5.0 \\
\hline 13S03W34BAA1 & 333110 & 912539 & $2 / 26 / 2004$ & 39.77 & $3 / 11 / 2008$ & 40.74 & -1.0 \\
\hline 13S03W34CAA1 & 333136 & 912336 & $2 / 26 / 2004$ & 36.52 & $3 / 11 / 2008$ & 37.92 & -1.4 \\
\hline 13S03W35BAC1 & 333154 & 912246 & $2 / 26 / 2004$ & 38.87 & $3 / 11 / 2008$ & 41.29 & -2.4 \\
\hline 14S02W09BDD1 & 332859 & 911729 & $5 / 07 / 2004$ & 29 & $3 / 26 / 2008$ & 30 & -1.0 \\
\hline 14S03W32CDB2 & 332613 & 912551 & $2 / 26 / 2004$ & 34.93 & $3 / 11 / 2008$ & 35.76 & -0.8 \\
\hline 15S02W20DDC1 & 332227 & 911920 & $4 / 28 / 2004$ & 30 & $3 / 26 / 2008$ & 32 & -2.0 \\
\hline
\end{tabular}


Appendix 2. Information pertaining to water levels measured in 2004 and 2008 in the Mississippi River Valley alluvial aquifer in eastern Arkansas.-Continued

[USGS, U.S. Geological Survey; NRCS, Natural Resources Conservation Service, ANRC, Arkansas Natural Resources Commission; Horizontal coordinate information is referenced to the North American Datum of 1983 (NAD83); *, control wells for duplicate measurements by USGS and NRCS for quality assurance]

\begin{tabular}{|c|c|c|c|c|c|c|c|}
\hline Station name & $\begin{array}{l}\text { Latitude } \\
\text { (degrees, } \\
\text { minutes, } \\
\text { seconds) }\end{array}$ & $\begin{array}{l}\text { Longitude } \\
\text { (degrees, } \\
\text { minutes, } \\
\text { seconds) }\end{array}$ & $\begin{array}{l}2004 \text { water- } \\
\text { level date }\end{array}$ & $\begin{array}{c}2004 \text { depth } \\
\text { to water } \\
\text { (feet below } \\
\text { land-surface } \\
\text { datum) }\end{array}$ & $\begin{array}{l}2008 \text { water- } \\
\text { level date }\end{array}$ & $\begin{array}{c}2008 \text { depth } \\
\text { to water } \\
\text { (feet below } \\
\text { land-surface } \\
\text { datum) }\end{array}$ & $\begin{array}{c}\text { Water-level } \\
\text { difference } \\
2004 \text { to } 2008 \\
\text { (feet) }\end{array}$ \\
\hline \multicolumn{8}{|c|}{ Chicot County-Continued } \\
\hline 16S03W11ADC1 & 331920 & 912234 & $2 / 27 / 2004$ & 28.51 & $3 / 11 / 2008$ & 29.61 & -1.1 \\
\hline 17S01E17CDA1 & 331259 & 910716 & $2 / 26 / 2004$ & 21.77 & $3 / 11 / 2008$ & 21 & 0.8 \\
\hline 17S01E18ADA1 & 331326 & 910758 & $2 / 26 / 2004$ & 11.24 & $3 / 11 / 2008$ & 11.53 & -0.3 \\
\hline 17S01W06BCC1 & 331501 & 911505 & $2 / 27 / 2004$ & 22.17 & $3 / 11 / 2008$ & 21.57 & 0.6 \\
\hline 17S02W10AAA1 & 331429 & 911712 & $2 / 27 / 2004$ & 27.04 & $3 / 11 / 2008$ & 27.36 & -0.3 \\
\hline 17S03W18CBC1 & 331257 & 912736 & $4 / 28 / 2004$ & 33 & $3 / 26 / 2008$ & 35 & -2.0 \\
\hline 17S03W28DBA1 & 331127 & 912441 & $2 / 27 / 2004$ & 24.61 & $3 / 11 / 2008$ & 24.74 & -0.1 \\
\hline 18S01W19DAB1 & 330709 & 911423 & $2 / 26 / 2004$ & 12.18 & $3 / 11 / 2008$ & 13.95 & -1.8 \\
\hline 18S01W33BAD1 & 330543 & 911245 & $4 / 28 / 2004$ & 13 & $3 / 26 / 2008$ & 14 & -1.0 \\
\hline 18S03W22ABA2 & 330728 & 912341 & $2 / 26 / 2004$ & 12.53 & $3 / 11 / 2008$ & 11.17 & 1.4 \\
\hline 19S01W17BCC1 & 330250 & 911406 & $2 / 26 / 2004$ & 19.19 & $3 / 11 / 2008$ & 20.57 & -1.4 \\
\hline 19S03W14ABB1 & 330304 & 912251 & $2 / 26 / 2004$ & 22.92 & $3 / 11 / 2008$ & 23.99 & -1.1 \\
\hline \multicolumn{8}{|c|}{ Clay County } \\
\hline 18N08E03DAB1 & 361323 & 901153 & $3 / 30 / 2004$ & 6.84 & $4 / 09 / 2008$ & 4.29 & 2.6 \\
\hline 18N08E11BAA1 & 361253 & 901117 & $4 / 14 / 2004$ & 6.8 & $4 / 02 / 2008$ & 4 & 2.8 \\
\hline 19N03E24AAA1 & 361655 & 904157 & $3 / 30 / 2004$ & 18.18 & $4 / 09 / 2008$ & 20.96 & -2.8 \\
\hline 19N04E11DAA1 & 361805 & 903621 & $4 / 14 / 2004$ & 22.6 & $4 / 02 / 2008$ & 17.8 & 4.8 \\
\hline 19N04E19AAA1 & 361654 & 904050 & $3 / 30 / 2004$ & 29.03 & $4 / 09 / 2008$ & 31.56 & -2.5 \\
\hline 19N04E19BAA1 & 361649 & 904125 & $4 / 14 / 2004$ & 21.5 & $4 / 06 / 2008$ & 23.2 & -1.7 \\
\hline 19N05E15BBD1 & 361716 & 903152 & $4 / 14 / 2004$ & 32.5 & $4 / 02 / 2008$ & 39 & -6.5 \\
\hline 19N06E18DBC1 & 361642 & 902815 & $4 / 14 / 2004$ & 32.5 & $4 / 02 / 2008$ & 38.9 & -6.4 \\
\hline 19N07E25BCB1 & 361519 & 901700 & $4 / 14 / 2004$ & 16.6 & $4 / 02 / 2008$ & 13.1 & 3.5 \\
\hline 19N08E08DCA1 & 361729 & 901402 & $4 / 14 / 2004$ & 8 & $4 / 02 / 2008$ & 4 & 4.0 \\
\hline 19N09E19CDC1 & 361539 & 900908 & $4 / 14 / 2004$ & 7.5 & $4 / 02 / 2008$ & 4.5 & 3.0 \\
\hline 20N03E25BAA1 & 362112 & 904225 & $4 / 14 / 2004$ & 21.8 & $4 / 06 / 2008$ & 23 & -1.2 \\
\hline 20N04E03ADA1 & 362425 & 903725 & $4 / 14 / 2004$ & 16.9 & $4 / 08 / 2008$ & 16 & 0.9 \\
\hline 20N05E22CAD1 & 362118 & 903132 & $4 / 14 / 2004$ & 27 & $4 / 02 / 2008$ & 29.5 & -2.5 \\
\hline 20N05E30CAC1 & 362003 & 903454 & $4 / 14 / 2004$ & 16.9 & $4 / 06 / 2008$ & 17.4 & -0.5 \\
\hline 20N05E34DBA1 & 361939 & 903117 & $3 / 30 / 2004$ & 27.63 & $4 / 09 / 2008$ & 31.78 & -4.2 \\
\hline 20N06E09BBA1 & 362327 & 902620 & $4 / 14 / 2004$ & 19.8 & $3 / 26 / 2008$ & 22.5 & -2.7 \\
\hline 20N06E28CCD1 & 362005 & 902630 & $4 / 14 / 2004$ & 27 & $4 / 02 / 2008$ & 30.8 & -3.8 \\
\hline 20N08E22BDC1 & 362111 & 901220 & $4 / 14 / 2004$ & 8.5 & $4 / 02 / 2008$ & 6.5 & 2.0 \\
\hline 20N09E09ABC1 & 362306 & 900642 & $4 / 14 / 2004$ & 8 & $4 / 02 / 2008$ & 3 & 5.0 \\
\hline 20N09E33DDC1 & 361904 & 900628 & $4 / 14 / 2004$ & 6.9 & $4 / 02 / 2008$ & 5 & 1.9 \\
\hline
\end{tabular}


Appendix 2. Information pertaining to water levels measured in 2004 and 2008 in the Mississippi River Valley alluvial aquifer in eastern Arkansas.-Continued

[USGS, U.S. Geological Survey; NRCS, Natural Resources Conservation Service, ANRC, Arkansas Natural Resources Commission; Horizontal coordinate information is referenced to the North American Datum of 1983 (NAD83); *, control wells for duplicate measurements by USGS and NRCS for quality assurance]

\begin{tabular}{|c|c|c|c|c|c|c|c|}
\hline Station name & $\begin{array}{c}\text { Latitude } \\
\text { (degrees, } \\
\text { minutes, } \\
\text { seconds) }\end{array}$ & $\begin{array}{c}\text { Longitude } \\
\text { (degrees, } \\
\text { minutes, } \\
\text { seconds) }\end{array}$ & $\begin{array}{l}2004 \text { water- } \\
\text { level date }\end{array}$ & $\begin{array}{l}2004 \text { depth } \\
\text { to water } \\
\text { (feet below } \\
\text { land-surface } \\
\text { datum) }\end{array}$ & $\begin{array}{l}2008 \text { water- } \\
\text { level date }\end{array}$ & $\begin{array}{l}2008 \text { depth } \\
\text { to water } \\
\text { (feet below } \\
\text { land-surface } \\
\text { datum) }\end{array}$ & $\begin{array}{c}\text { Water-level } \\
\text { difference } \\
2004 \text { to } 2008 \\
\text { (feet) }\end{array}$ \\
\hline \multicolumn{8}{|c|}{ Clay County-Continued } \\
\hline 21N03E15CBC1 & 362738 & 904453 & $4 / 14 / 2004$ & 12.1 & $4 / 28 / 2008$ & 5 & 7.1 \\
\hline 21N03E36CDD1 & 362450 & 904214 & $4 / 14 / 2004$ & 18.1 & $4 / 08 / 2008$ & 11.1 & 7.0 \\
\hline 21N04E09DBC1 & 362828 & 903853 & $4 / 14 / 2004$ & 11 & $4 / 08 / 2008$ & 10 & 1.0 \\
\hline 21N05E17ABB1 & 362755 & 903329 & $3 / 30 / 2004$ & 20.32 & $4 / 09 / 2008$ & 23.92 & -3.6 \\
\hline 21N05E22BAB1 & 362704 & 903132 & $4 / 14 / 2004$ & 6.5 & $4 / 08 / 2008$ & 5.8 & 0.7 \\
\hline 21N06E11BBB1 & 362839 & 902421 & $4 / 14 / 2004$ & 11.9 & $4 / 02 / 2008$ & 15.2 & -3.3 \\
\hline 21N06E28BB1 & 362605 & 902608 & $3 / 30 / 2004$ & 17.03 & $4 / 09 / 2008$ & 19.38 & -2.4 \\
\hline 21N07E01DDC1 & 362835 & 901607 & $4 / 14 / 2004$ & 18.5 & $4 / 02 / 2008$ & 33.2 & -14.7 \\
\hline 21N07E19BDA1 & 362640 & 902148 & $4 / 14 / 2004$ & 18 & $3 / 26 / 2008$ & 24.8 & -6.8 \\
\hline 21N08E18CCC1 & 362651 & 901550 & $3 / 30 / 2004$ & 30.87 & $4 / 09 / 2008$ & 40.71 & -9.8 \\
\hline 21N08E36ABB1 & 362502 & 900958 & $3 / 30 / 2004$ & 1.63 & $4 / 09 / 2008$ & 2.68 & -1.1 \\
\hline 21N09E31BDA1 & 362447 & 900851 & $4 / 14 / 2004$ & 5.4 & $4 / 2 / 2008$ & 1 & 4.4 \\
\hline \multicolumn{8}{|c|}{ Craighead County } \\
\hline 13N01E03AAA1 & 354739 & 905753 & $3 / 16 / 2004$ & 52.2 & $3 / 17 / 2008$ & 56.6 & -4.4 \\
\hline $13 \mathrm{~N} 01 \mathrm{E} 21 \mathrm{CAB}$ & 354434 & 905945 & $3 / 16 / 2004$ & 59 & $3 / 17 / 2008$ & 65.5 & -6.5 \\
\hline 13N01E23CAB1 & 354430 & 905736 & $3 / 17 / 2004$ & 64.5 & $3 / 17 / 2008$ & 70.1 & -5.6 \\
\hline 13N01E23DAA1 & 354435 & 905652 & $3 / 25 / 2004$ & 69.39 & $4 / 10 / 2008$ & 71.08 & -1.7 \\
\hline $13 \mathrm{~N} 02 \mathrm{E} 02 \mathrm{AAB} 1$ & 354731 & 905032 & $3 / 16 / 2004$ & 85 & $3 / 17 / 2008$ & 93.8 & -8.8 \\
\hline 13N02E03AAA1 & 354733 & 905129 & $3 / 16 / 2004$ & 83.8 & $3 / 17 / 2008$ & 88.7 & -4.9 \\
\hline 13N03E23CDA1 & 354419 & 904434 & $3 / 16 / 2004$ & 78.8 & $3 / 12 / 2008$ & 81.7 & -2.9 \\
\hline 13N03E29AAA1 & 354403 & 904713 & $3 / 25 / 2004$ & 102.05 & $4 / 10 / 2008$ & 105.76 & -3.7 \\
\hline 13N03E35AAA1 & 354308 & 904401 & $3 / 16 / 2004$ & 93.5 & $3 / 12 / 2008$ & 97.5 & -4.0 \\
\hline 13N04E12ABB1 & 354635 & 903656 & $3 / 25 / 2004$ & 23.46 & $4 / 10 / 2008$ & 24.51 & -1.1 \\
\hline 13N04E15DBA1 & 354521 & 903857 & $3 / 09 / 2004$ & 25.2 & $3 / 12 / 2008$ & 26.6 & -1.4 \\
\hline 13N04E26BCC1 & 354340 & 903829 & $3 / 09 / 2004$ & 27.4 & $3 / 12 / 2008$ & 26.6 & 0.8 \\
\hline 13N05E02CCC1 & 354648 & 903202 & $3 / 09 / 2004$ & 9 & $3 / 12 / 2008$ & 13.5 & -4.5 \\
\hline 13N05E06DCC1 & 354637 & 903547 & $3 / 09 / 2004$ & 20 & $3 / 12 / 2008$ & 20.4 & -0.4 \\
\hline 13N05E22BAD1 & 354449 & 903243 & $3 / 25 / 2004$ & 13.11 & $4 / 10 / 2008$ & 13.23 & -0.1 \\
\hline 13N05E24BAC1 & 354451 & 903045 & $3 / 09 / 2004$ & 5.5 & $3 / 12 / 2008$ & 8.8 & -3.3 \\
\hline 13N06E21AAA1 & 354450 & 902701 & $3 / 09 / 2004$ & 7 & $3 / 12 / 2008$ & 8.5 & -1.5 \\
\hline 13N07E02CAB1 & 354642 & 901901 & $3 / 16 / 2004$ & 5 & $3 / 12 / 2008$ & 10.5 & -5.5 \\
\hline 13N07E05ABB1 & 354716 & 902158 & $3 / 16 / 2004$ & 5.5 & $3 / 12 / 2008$ & 4.4 & 1.1 \\
\hline 13N07E20BBA1 & 354440 & 902216 & $3 / 25 / 2004$ & 3.9 & $4 / 10 / 2008$ & 1.95 & 2.0 \\
\hline
\end{tabular}


Appendix 2. Information pertaining to water levels measured in 2004 and 2008 in the Mississippi River Valley alluvial aquifer in eastern Arkansas.-Continued

[USGS, U.S. Geological Survey; NRCS, Natural Resources Conservation Service, ANRC, Arkansas Natural Resources Commission; Horizontal coordinate information is referenced to the North American Datum of 1983 (NAD83); *, control wells for duplicate measurements by USGS and NRCS for quality assurance]

\begin{tabular}{|c|c|c|c|c|c|c|c|}
\hline Station name & $\begin{array}{l}\text { Latitude } \\
\text { (degrees, } \\
\text { minutes, } \\
\text { seconds) }\end{array}$ & $\begin{array}{l}\text { Longitude } \\
\text { (degrees, } \\
\text { minutes, } \\
\text { seconds) }\end{array}$ & $\begin{array}{l}2004 \text { water- } \\
\text { level date }\end{array}$ & $\begin{array}{l}2004 \text { depth } \\
\text { to water } \\
\text { (feet below } \\
\text { land-surface } \\
\text { datum) }\end{array}$ & $\begin{array}{l}2008 \text { water- } \\
\text { level date }\end{array}$ & $\begin{array}{l}2008 \text { depth } \\
\text { to water } \\
\text { (feet below } \\
\text { land-surface } \\
\text { datum) }\end{array}$ & $\begin{array}{c}\text { Water-level } \\
\text { difference } \\
2004 \text { to } 2008 \\
\text { (feet) }\end{array}$ \\
\hline \multicolumn{8}{|c|}{ Craighead County-Continued } \\
\hline 13N07E35BCD1 & 354233 & 901837 & $3 / 16 / 2004$ & 8 & $3 / 12 / 2008$ & 12.9 & -4.9 \\
\hline 14N01E03ACB1 & 355246 & 905816 & $3 / 17 / 2004$ & 48.1 & $3 / 17 / 2008$ & 51.5 & -3.4 \\
\hline 14N01E10BAB1 & 355204 & 905828 & $3 / 17 / 2004$ & 50.1 & $3 / 17 / 2008$ & 54 & -3.9 \\
\hline 14N01E31DCA1 & 354817 & 910121 & $3 / 17 / 2004$ & 56.7 & $3 / 17 / 2008$ & 61.9 & -5.2 \\
\hline 14N02E18BDD1 & 355041 & 905419 & $3 / 25 / 2004$ & 50.25 & $4 / 10 / 2008$ & 53.57 & -3.3 \\
\hline 14N02E22AAA1 & 355007 & 905129 & $3 / 17 / 2004$ & 71 & $3 / 17 / 2008$ & 78 & -7.0 \\
\hline 14N05E25ABB1 & 354921 & 903025 & $3 / 25 / 2004$ & 18.14 & $4 / 10 / 2008$ & 18.58 & -0.4 \\
\hline 14N06E06BAA1 & 355234 & 902934 & $3 / 16 / 2004$ & 21 & $3 / 12 / 2008$ & 21.5 & -0.5 \\
\hline 14N06E27AAB1 & 354911 & 902559 & $3 / 25 / 2004$ & 2.45 & $4 / 10 / 2008$ & -0.25 & 2.7 \\
\hline 14N07E14DDC1 & 354956 & 901831 & $3 / 08 / 2004$ & 4.5 & $3 / 12 / 2008$ & 13.1 & -8.6 \\
\hline 14N07E26DBB1 & 354834 & 901843 & $3 / 25 / 2004$ & 5.35 & $4 / 10 / 2008$ & 2.68 & 2.7 \\
\hline 15N02E12DCB1 & 355626 & 904930 & $3 / 17 / 2004$ & 31.7 & $3 / 17 / 2008$ & 36.5 & -4.8 \\
\hline 15N03E19ADA1 & 355502 & 904802 & $3 / 25 / 2004$ & 47.93 & $4 / 10 / 2008$ & 51.27 & -3.3 \\
\hline 15N05E22BAB1 & 355513 & 903241 & $3 / 16 / 2004$ & 38 & $3 / 12 / 2008$ & 31.8 & 6.2 \\
\hline 15N06E04BAD1 & 355744 & 902706 & $3 / 12 / 2004$ & 11 & $3 / 12 / 2008$ & 17.4 & -6.4 \\
\hline 15N06E20DDD1 & 355426 & 902739 & $3 / 25 / 2004$ & 8.62 & $4 / 10 / 2008$ & 9.37 & -0.8 \\
\hline 15N07E35DCB1 & 355241 & 901831 & $3 / 08 / 2004$ & 6.5 & $3 / 12 / 2008$ & 13.9 & -7.4 \\
\hline \multicolumn{8}{|c|}{ Crittenden County } \\
\hline 04N07E21AAD1 & 345644 & 902121 & $3 / 24 / 2004$ & 9.98 & $4 / 03 / 2008$ & 10.1 & -0.1 \\
\hline 05N07E08BDC1 & 350407 & 902234 & $4 / 13 / 2004$ & 22 & $4 / 07 / 2008$ & 26 & -4.0 \\
\hline 05N07E28CBA1 & 350121 & 902140 & $3 / 24 / 2004$ & 16.98 & $4 / 03 / 2008$ & 17.08 & -0.1 \\
\hline 05N07E34BAB1 & 350059 & 902030 & $3 / 24 / 2004$ & 14.89 & $4 / 03 / 2008$ & 16.27 & -1.4 \\
\hline 05N07E34CDD1 & 350010 & 902028 & $4 / 13 / 2004$ & 19 & $4 / 07 / 2008$ & 9.9 & 9.1 \\
\hline 05N08E11CCD2 & 350345 & 901308 & $3 / 24 / 2004$ & 26.06 & $4 / 03 / 2008$ & 25.27 & 0.8 \\
\hline 06N07E14ABA1 & 350848 & 901858 & $4 / 13 / 2004$ & 25 & $4 / 07 / 2008$ & 23.6 & 1.4 \\
\hline 07N06E29CBC1 & 351152 & 902914 & $4 / 13 / 2004$ & 37 & $4 / 08 / 2008$ & 40.11 & -3.1 \\
\hline 07N07E31CCC1 & 351042 & 902359 & $3 / 24 / 2004$ & 31.56 & $4 / 03 / 2008$ & 36.48 & -4.9 \\
\hline 07N08E04BBD1 & 351538 & 901505 & $4 / 15 / 2004$ & 19 & $3 / 26 / 2008$ & 22.9 & -3.9 \\
\hline 07N09E05CDD1 & 351453 & 900934 & $3 / 24 / 2004$ & 14.34 & $4 / 03 / 2008$ & 14.83 & -0.5 \\
\hline 08N06E01DCC1 & 352021 & 902408 & $4 / 13 / 2004$ & 32 & $3 / 26 / 2008$ & 33.1 & -1.1 \\
\hline 08N07E13CCC2 & 351828 & 901812 & $3 / 24 / 2004$ & 28.25 & $4 / 03 / 2008$ & 31.43 & -3.2 \\
\hline 08N07E14DAA2 & 351854 & 901833 & $3 / 24 / 2004$ & 29.2 & $4 / 03 / 2008$ & 31.82 & -2.6 \\
\hline 08N07E32DAA1 & 351618 & 902146 & $4 / 13 / 2004$ & 26 & $4 / 07 / 2008$ & 31.4 & -5.4 \\
\hline 08N08E06ABB1 & 352103 & 901644 & $4 / 13 / 2004$ & 27.5 & $3 / 26 / 2008$ & 31.3 & -3.8 \\
\hline
\end{tabular}


Appendix 2. Information pertaining to water levels measured in 2004 and 2008 in the Mississippi River Valley alluvial aquifer in eastern Arkansas.-Continued

[USGS, U.S. Geological Survey; NRCS, Natural Resources Conservation Service, ANRC, Arkansas Natural Resources Commission; Horizontal coordinate information is referenced to the North American Datum of 1983 (NAD83); *, control wells for duplicate measurements by USGS and NRCS for quality assurance]

\begin{tabular}{|c|c|c|c|c|c|c|c|}
\hline Station name & $\begin{array}{c}\text { Latitude } \\
\text { (degrees, } \\
\text { minutes, } \\
\text { seconds) }\end{array}$ & $\begin{array}{l}\text { Longitude } \\
\text { (degrees, } \\
\text { minutes, } \\
\text { seconds) }\end{array}$ & $\begin{array}{l}2004 \text { water- } \\
\text { level date }\end{array}$ & $\begin{array}{l}2004 \text { depth } \\
\text { to water } \\
\text { (feet below } \\
\text { land-surface } \\
\text { datum) }\end{array}$ & $\begin{array}{l}2008 \text { water- } \\
\text { level date }\end{array}$ & $\begin{array}{l}2008 \text { depth } \\
\text { to water } \\
\text { (feet below } \\
\text { land-surface } \\
\text { datum) }\end{array}$ & $\begin{array}{c}\text { Water-level } \\
\text { difference } \\
2004 \text { to } 2008 \\
\text { (feet) }\end{array}$ \\
\hline \multicolumn{8}{|c|}{ Crittenden County-Continued } \\
\hline 09N07E02CDB1 & 352537 & 901905 & $4 / 13 / 2004$ & 33 & $3 / 26 / 2008$ & 33.5 & -0.5 \\
\hline 09N07E10DDA1 & 352448 & 901925 & $3 / 24 / 2004$ & 27.26 & $4 / 03 / 2008$ & 29.29 & -2.0 \\
\hline 09N07E31BAB1* & 352160 & 902327 & $3 / 24 / 2004$ & 32.31 & $3 / 26 / 2008$ & 32.1 & 0.2 \\
\hline 09N07E31BAB1* & 352160 & 902327 & $4 / 13 / 2004$ & 31 & $4 / 03 / 2008$ & 34.06 & -3.1 \\
\hline 09N08E04CDC1 & 352527 & 901444 & $4 / 13 / 2004$ & 24 & $3 / 26 / 2008$ & 27 & -3.0 \\
\hline \multicolumn{8}{|c|}{ Cross County } \\
\hline 06N02E11BDB1 & 350934 & 905132 & $4 / 15 / 2004$ & 61 & $4 / 14 / 2008$ & 69.5 & -8.5 \\
\hline 06N02E12AAA1 & 350934 & 904952 & $4 / 15 / 2004$ & 79 & $4 / 14 / 2008$ & 81 & -2.0 \\
\hline 07N01E05CDA1 & 351518 & 910049 & $3 / 23 / 2004$ & 71.75 & $4 / 04 / 2008$ & 76.38 & -4.6 \\
\hline 07N01E05DCA1 & 351514 & 910033 & $4 / 15 / 2004$ & 72 & $4 / 08 / 2008$ & 77.5 & -5.5 \\
\hline 07N01E06CAA1 & 351530 & 910154 & $4 / 08 / 2004$ & 71 & $4 / 08 / 2008$ & 75 & -4.0 \\
\hline 07N01E11AAA1 & 351501 & 905705 & $3 / 23 / 2004$ & 74.77 & $4 / 02 / 2008$ & 78.62 & -3.9 \\
\hline 07N02E29DDC1 & 351138 & 905409 & $3 / 23 / 2004$ & 70.48 & $4 / 02 / 2008$ & 74.14 & -3.7 \\
\hline 07N03E05ADA1 & 351549 & 904739 & $3 / 23 / 2004$ & 120.85 & $4 / 08 / 2008$ & 112.23 & 8.6 \\
\hline 07N03E32DCC1 & 351045 & 904810 & $3 / 23 / 2004$ & 95.88 & $4 / 02 / 2008$ & 97.8 & -1.9 \\
\hline 07N05E19CCC1 & 351238 & 903645 & $3 / 23 / 2004$ & 34.91 & $4 / 02 / 2008$ & 39.34 & -4.4 \\
\hline 07N05E24CCC1 & 351232 & 903121 & $4 / 15 / 2004$ & 35 & $4 / 03 / 2008$ & 37.6 & -2.6 \\
\hline 07N05E25ABA1 & 351229 & 903045 & $3 / 23 / 2004$ & 35.81 & $4 / 07 / 2008$ & 38.94 & -3.1 \\
\hline 08N01E16DBB1 & 351855 & 905933 & $4 / 08 / 2004$ & 84 & $4 / 08 / 2008$ & 87 & -3.0 \\
\hline 08N02E12DCC1 & 351938 & 905002 & $4 / 15 / 2004$ & 88 & $4 / 14 / 2008$ & 92 & -4.0 \\
\hline 08N02E17AAA1 & 351923 & 905354 & $4 / 08 / 2004$ & 82 & $4 / 08 / 2008$ & 90 & -8.0 \\
\hline 08N05E32ADD1 & 351632 & 903440 & $3 / 23 / 2004$ & 28.47 & $4 / 03 / 2008$ & 31.47 & -3.0 \\
\hline 09N01E04ACD1 & 352608 & 905914 & $4 / 08 / 2004$ & 85 & $4 / 08 / 2008$ & 90 & -5.0 \\
\hline 09N01E33BBA2 & 352203 & 910001 & $3 / 23 / 2004$ & 78.4 & $4 / 04 / 2008$ & 83.67 & -5.3 \\
\hline 09N01E36AAB1 & 352155 & 905605 & $4 / 15 / 2004$ & 83 & $4 / 08 / 2008$ & 91.5 & -8.5 \\
\hline 09N02E20AAA1 & 352402 & 905342 & $4 / 08 / 2004$ & 91 & $4 / 08 / 2008$ & 94 & -3.0 \\
\hline 09N02E30CBB1 & 352243 & 905551 & $4 / 08 / 2004$ & 87 & $4 / 08 / 2008$ & 90 & -3.0 \\
\hline 09N03E17CDD1 & 352422 & 904753 & $4 / 08 / 2004$ & 102 & $4 / 14 / 2008$ & 105.5 & -3.5 \\
\hline 09N03E17DDC1 & 352409 & 904726 & $3 / 23 / 2004$ & 103.96 & $4 / 04 / 2008$ & 107.77 & -3.8 \\
\hline 09N05E32BCB1 & 352151 & 903525 & $4 / 15 / 2004$ & 35 & $4 / 09 / 2008$ & 30 & 5.0 \\
\hline 09N05E32BDB1 & 352151 & 903512 & $3 / 23 / 2004$ & 29.12 & $4 / 03 / 2008$ & 32.17 & -3.1 \\
\hline \multicolumn{8}{|c|}{ Desha County } \\
\hline 07S01E19ABA1 & 340428 & 910303 & $4 / 13 / 2004$ & 14 & $3 / 25 / 2008$ & 22.5 & -8.5 \\
\hline 08S03W33ABD1 & 335803 & 912338 & $3 / 02 / 2004$ & 5.32 & $3 / 13 / 2008$ & 6.93 & -1.6 \\
\hline
\end{tabular}


Appendix 2. Information pertaining to water levels measured in 2004 and 2008 in the Mississippi River Valley alluvial aquifer in eastern Arkansas.-Continued

[USGS, U.S. Geological Survey; NRCS, Natural Resources Conservation Service, ANRC, Arkansas Natural Resources Commission; Horizontal coordinate information is referenced to the North American Datum of 1983 (NAD83); *, control wells for duplicate measurements by USGS and NRCS for quality assurance]

\begin{tabular}{|c|c|c|c|c|c|c|c|}
\hline Station name & $\begin{array}{l}\text { Latitude } \\
\text { (degrees, } \\
\text { minutes, } \\
\text { seconds) }\end{array}$ & $\begin{array}{c}\text { Longitude } \\
\text { (degrees, } \\
\text { minutes, } \\
\text { seconds) }\end{array}$ & $\begin{array}{l}2004 \text { water- } \\
\text { level date }\end{array}$ & $\begin{array}{l}2004 \text { depth } \\
\text { to water } \\
\text { (feet below } \\
\text { land-surface } \\
\text { datum) }\end{array}$ & $\begin{array}{l}2008 \text { water- } \\
\text { level date }\end{array}$ & $\begin{array}{l}2008 \text { depth } \\
\text { to water } \\
\text { (feet below } \\
\text { land-surface } \\
\text { datum) }\end{array}$ & $\begin{array}{c}\text { Water-level } \\
\text { difference } \\
2004 \text { to } 2008 \\
\text { (feet) }\end{array}$ \\
\hline \multicolumn{8}{|c|}{ Desha County-Continued } \\
\hline 09S01W08BDA1 & 335608 & 911234 & $4 / 30 / 2004$ & 25 & $4 / 01 / 2008$ & 30 & -5.0 \\
\hline 09S01W15CBB1 & 335501 & 911055 & $4 / 30 / 2004$ & 35 & $4 / 01 / 2008$ & 39 & -4.0 \\
\hline 09S02W26DDC1 & 335257 & 911530 & $3 / 02 / 2004$ & 30.47 & $5 / 13 / 2008$ & 31.68 & -1.2 \\
\hline 09S03W05BAC1 & 335704 & 912506 & $4 / 30 / 2004$ & 38 & $4 / 01 / 2008$ & 36 & 2.0 \\
\hline 09S03W13BAB1 & 335500 & 911922 & $4 / 30 / 2004$ & 31 & $4 / 01 / 2008$ & 35 & -4.0 \\
\hline 09S03W17DCB1 & 335448 & 912457 & $3 / 02 / 2004$ & 33.82 & $3 / 13 / 2008$ & 33.68 & 0.1 \\
\hline 09S04W06BCA1 & 335756 & 913243 & $3 / 02 / 2004$ & 33.75 & $3 / 13 / 2008$ & 36.43 & -2.7 \\
\hline 10S01W23CDA1 & 335305 & 911032 & $4 / 30 / 2004$ & 22 & $4 / 01 / 2008$ & 31 & -9.0 \\
\hline 10S02W11ADD1 & 335045 & 911517 & $4 / 30 / 2004$ & 28 & $4 / 01 / 2008$ & 31 & -3.0 \\
\hline 10S02W24DBC1 & 334850 & 911453 & $3 / 02 / 2004$ & 25.88 & $3 / 12 / 2008$ & 26.18 & -0.3 \\
\hline 10S03W26CAA1 & 334806 & 912145 & $3 / 02 / 2004$ & 45.12 & $3 / 12 / 2008$ & 47.43 & -2.3 \\
\hline 11S02W15ADD1 & 334446 & 911635 & $4 / 30 / 2004$ & 33 & $4 / 01 / 2008$ & 36 & -3.0 \\
\hline 11S03W16CBA1 & 334439 & 912433 & $4 / 30 / 2004$ & 32 & $4 / 01 / 2008$ & 36 & -4.0 \\
\hline 11S03W31BBA1 & 334228 & 912651 & $3 / 02 / 2004$ & 34.18 & $3 / 12 / 2008$ & 37.27 & -3.1 \\
\hline 12S01W33BAA1 & 333718 & 911205 & $3 / 02 / 2004$ & 24.55 & $3 / 13 / 2008$ & 25.21 & -0.7 \\
\hline 13S02W17ADA1 & 333421 & 911858 & $4 / 30 / 2004$ & 44 & $4 / 01 / 2008$ & 46 & -2.0 \\
\hline 13S02W27CAC1 & 333224 & 911735 & $3 / 02 / 2004$ & 31.92 & $3 / 12 / 2008$ & 32.43 & -0.5 \\
\hline 13S02W32DBD1 & 333126 & 911917 & $4 / 30 / 2004$ & 38 & $4 / 01 / 2008$ & 40 & -2.0 \\
\hline 13S03W10DAA1 & 333506 & 912302 & $3 / 02 / 2004$ & 45.95 & $3 / 12 / 2008$ & 49.17 & -3.2 \\
\hline 13S03W11CAB1 & 333503 & 912241 & $4 / 30 / 2004$ & 51 & $4 / 01 / 2008$ & 52 & -1.0 \\
\hline \multicolumn{8}{|c|}{ Drew County } \\
\hline 11S04W08DBA1 & 334532 & 913136 & $3 / 01 / 2004$ & 23.13 & $3 / 12 / 2008$ & 26.08 & -3.0 \\
\hline 11S05W08CCC1 & 334546 & 913837 & $3 / 01 / 2004$ & 35.79 & $3 / 12 / 2008$ & 37.47 & -1.7 \\
\hline 12S04W03ABB1 & 334134 & 912946 & $3 / 01 / 2004$ & 24.11 & $3 / 12 / 2008$ & 26.02 & -1.9 \\
\hline 12S04W25DBB1 & 333739 & 912738 & $5 / 04 / 2004$ & 34 & $3 / 17 / 2008$ & 36 & -2.0 \\
\hline 13S04W09ACD1 & 333512 & 913034 & $5 / 04 / 2004$ & 16.4 & $3 / 17 / 2008$ & 20 & -3.6 \\
\hline 13S04W33BAA1 & 333206 & 913100 & $3 / 01 / 2004$ & 19.03 & $3 / 12 / 2008$ & 19.32 & -0.3 \\
\hline 13S05W29ADA1 & 333248 & 913747 & $3 / 01 / 2004$ & 30.5 & $3 / 12 / 2008$ & 46.85 & -16.4 \\
\hline 13S06W03DDC1 & 333545 & 914202 & $3 / 01 / 2004$ & 61.06 & $3 / 12 / 2008$ & 64.89 & -3.8 \\
\hline 13S06W21DAA1 & 333324 & 914258 & $5 / 05 / 2004$ & 89 & $3 / 17 / 2008$ & 72 & 17.0 \\
\hline 14S04W03ADD1 & 333050 & 912929 & $5 / 04 / 2004$ & 25 & $3 / 17 / 2008$ & 28 & -3.0 \\
\hline 14S04W05CBC1 & 333042 & 913226 & $5 / 04 / 2004$ & 13 & $3 / 17 / 2008$ & 20 & -7.0 \\
\hline 15S04W13DAD1 & 332338 & 912730 & $4 / 28 / 2004$ & 35 & $3 / 25 / 2008$ & 42 & -7.0 \\
\hline
\end{tabular}


Appendix 2. Information pertaining to water levels measured in 2004 and 2008 in the Mississippi River Valley alluvial aquifer in eastern Arkansas.-Continued

[USGS, U.S. Geological Survey; NRCS, Natural Resources Conservation Service, ANRC, Arkansas Natural Resources Commission; Horizontal coordinate information is referenced to the North American Datum of 1983 (NAD83); *, control wells for duplicate measurements by USGS and NRCS for quality assurance]

\begin{tabular}{|c|c|c|c|c|c|c|c|}
\hline Station name & $\begin{array}{l}\text { Latitude } \\
\text { (degrees, } \\
\text { minutes, } \\
\text { seconds) }\end{array}$ & $\begin{array}{l}\text { Longitude } \\
\text { (degrees, } \\
\text { minutes, } \\
\text { seconds) }\end{array}$ & $\begin{array}{l}2004 \text { water- } \\
\text { level date }\end{array}$ & $\begin{array}{l}2004 \text { depth } \\
\text { to water } \\
\text { (feet below } \\
\text { land-surface } \\
\text { datum) }\end{array}$ & $\begin{array}{l}2008 \text { water- } \\
\text { level date }\end{array}$ & $\begin{array}{l}2008 \text { depth } \\
\text { to water } \\
\text { (feet below } \\
\text { land-surface } \\
\text { datum) }\end{array}$ & $\begin{array}{c}\text { Water-level } \\
\text { difference } \\
2004 \text { to } 2008 \\
\text { (feet) }\end{array}$ \\
\hline \multicolumn{8}{|c|}{ Greene County } \\
\hline 16N03E03BA1 & 360316 & 904516 & $3 / 30 / 2004$ & 29.04 & $4 / 09 / 2008$ & 33.17 & -4.1 \\
\hline 16N03E05BBB1 & 360316 & 904750 & $4 / 16 / 2004$ & 28.4 & $4 / 17 / 2008$ & 32.6 & -4.2 \\
\hline 16N03E16DDD1 & 360049 & 904547 & $4 / 16 / 2004$ & 34.8 & $4 / 17 / 2008$ & 28.1 & 6.7 \\
\hline 16N03E29ACC1 & 355926 & 904722 & $4 / 16 / 2004$ & 29.2 & $4 / 17 / 2008$ & 37.1 & -7.9 \\
\hline 16N06E03CCC1 & 360224 & 902626 & $3 / 29 / 2004$ & 40.39 & $4 / 09 / 2008$ & 41.19 & -0.8 \\
\hline 16N06E09ABB1 & 360215 & 902651 & $4 / 15 / 2004$ & 40.4 & $4 / 07 / 2008$ & 40.2 & 0.2 \\
\hline 16N06E21BAA1 & 360031 & 902705 & $4 / 15 / 2004$ & 25 & $4 / 07 / 2008$ & 27.6 & -2.6 \\
\hline 16N06E28ABB1 & 355938 & 902657 & $3 / 29 / 2004$ & 23.89 & $4 / 09 / 2008$ & 25.73 & -1.8 \\
\hline 17N03E02DCC1 & 360806 & 904352 & $4 / 16 / 2004$ & 31 & $4 / 17 / 2008$ & 30.8 & 0.2 \\
\hline 17N04E07AD1 & 360718 & 904122 & $4 / 16 / 2004$ & 36.1 & $4 / 17 / 2008$ & 38.1 & -2.0 \\
\hline 17N04E30CDC1 & 360409 & 904218 & $3 / 30 / 2004$ & 35.38 & $4 / 09 / 2008$ & 39.09 & -3.7 \\
\hline 17N06E15ABC1 & 360631 & 902546 & $4 / 15 / 2004$ & 38.2 & $4 / 17 / 2008$ & 30.9 & 7.3 \\
\hline 17N07E18ABB1 & 360638 & 902235 & $3 / 29 / 2004$ & 6.97 & $4 / 09 / 2008$ & 7.85 & -0.9 \\
\hline 18N03E24ACA1 & 361119 & 904216 & $4 / 16 / 2004$ & 29.8 & $4 / 17 / 2008$ & 35.1 & -5.3 \\
\hline 18N04E04AAC1 & 361356 & 903854 & $4 / 16 / 2004$ & 28.2 & $4 / 17 / 2008$ & 31.3 & -3.1 \\
\hline 18N04E21CBD1 & 361052 & 903725 & $3 / 30 / 2004$ & 53.9 & $4 / 09 / 2008$ & 58.57 & -4.7 \\
\hline 18N06E23ABB1 & 361109 & 902402 & $4 / 15 / 2004$ & 10.3 & $4 / 17 / 2008$ & 15.4 & -5.1 \\
\hline 18N07E17BAB1 & 361203 & 902105 & $4 / 15 / 2004$ & 8.3 & $4 / 07 / 2008$ & 8.6 & -0.3 \\
\hline 18N07E20BBA1 & 361110 & 902113 & $3 / 29 / 2004$ & 6.88 & $4 / 09 / 2008$ & 7.23 & -0.4 \\
\hline 19N03E26AD1 & 361601 & 904258 & $3 / 30 / 2004$ & 27.59 & $4 / 09 / 2008$ & 30.28 & -2.7 \\
\hline 19N03E33DDD1 & 361418 & 904516 & $4 / 16 / 2004$ & 33.8 & $4 / 17 / 2008$ & 36.5 & -2.7 \\
\hline 19N05E34AAD1 & 361437 & 903102 & $4 / 15 / 2004$ & 30.5 & $4 / 17 / 2008$ & 34.8 & -4.3 \\
\hline \multicolumn{8}{|c|}{ Independence County } \\
\hline 12N04W14DD1 & 353929 & 912236 & $3 / 31 / 2004$ & 24.71 & $4 / 16 / 2008$ & 10.54 & 14.2 \\
\hline 12N04W34CBB1 & 353720 & 912513 & $3 / 31 / 2004$ & 21.91 & $4 / 16 / 2008$ & 4.47 & 17.4 \\
\hline 12N05W36AAA1 & 353738 & 912827 & $3 / 31 / 2004$ & 23.75 & $4 / 16 / 2008$ & 6.41 & 17.3 \\
\hline \multicolumn{8}{|c|}{ Jackson County } \\
\hline 09N01W22ADD1 & 352332 & 910433 & $3 / 31 / 2004$ & 59.66 & $4 / 15 / 2008$ & 63.04 & -3.4 \\
\hline 09N02W32BBB1 & 352215 & 911344 & $4 / 01 / 2004$ & 29.2 & $3 / 27 / 2008$ & 32.2 & -3.0 \\
\hline 09N02W32CBB1 & 352152 & 911348 & $3 / 31 / 2004$ & 28.35 & $4 / 15 / 2008$ & 30.39 & -2.0 \\
\hline 10N02W29ABB1 & 352829 & 911312 & $3 / 31 / 2004$ & 25.83 & $4 / 15 / 2008$ & 28.47 & -2.6 \\
\hline 11N01W26AAD1* & 353330 & 910323 & $3 / 26 / 2004$ & 65.1 & $3 / 27 / 2008$ & 69.3 & -4.2 \\
\hline 11N01W26AAD1* & 353330 & 910323 & $3 / 31 / 2004$ & 65.25 & $4 / 15 / 2008$ & 68.29 & -3.0 \\
\hline 11N01W29AAD1 & 353339 & 910635 & $3 / 31 / 2004$ & 39.01 & $4 / 15 / 2008$ & 41.88 & -2.9 \\
\hline
\end{tabular}


Appendix 2. Information pertaining to water levels measured in 2004 and 2008 in the Mississippi River Valley alluvial aquifer in eastern Arkansas.-Continued

[USGS, U.S. Geological Survey; NRCS, Natural Resources Conservation Service, ANRC, Arkansas Natural Resources Commission; Horizontal coordinate information is referenced to the North American Datum of 1983 (NAD83); *, control wells for duplicate measurements by USGS and NRCS for quality assurance]

\begin{tabular}{|c|c|c|c|c|c|c|c|}
\hline Station name & $\begin{array}{l}\text { Latitude } \\
\text { (degrees, } \\
\text { minutes, } \\
\text { seconds) }\end{array}$ & $\begin{array}{l}\text { Longitude } \\
\text { (degrees, } \\
\text { minutes, } \\
\text { seconds) }\end{array}$ & $\begin{array}{l}2004 \text { water- } \\
\text { level date }\end{array}$ & $\begin{array}{l}2004 \text { depth } \\
\text { to water } \\
\text { (feet below } \\
\text { land-surface } \\
\text { datum) }\end{array}$ & $\begin{array}{l}2008 \text { water- } \\
\text { level date }\end{array}$ & $\begin{array}{l}2008 \text { depth } \\
\text { to water } \\
\text { (feet below } \\
\text { land-surface } \\
\text { datum) }\end{array}$ & $\begin{array}{c}\text { Water-level } \\
\text { difference } \\
2004 \text { to } 2008 \\
\text { (feet) }\end{array}$ \\
\hline \multicolumn{8}{|c|}{ Jackson County-Continued } \\
\hline 11N02W25BBD1 & 353322 & 910855 & $3 / 26 / 2004$ & 25.5 & $3 / 27 / 2008$ & 28.1 & -2.6 \\
\hline 12N01W11BCB1 & 354127 & 910416 & $4 / 06 / 2004$ & 36.6 & $3 / 27 / 2008$ & 40.7 & -4.1 \\
\hline 12N02W25ABB2 & 353910 & 910852 & $3 / 31 / 2004$ & 31.73 & $4 / 16 / 2008$ & 34.41 & -2.7 \\
\hline 13N01W20AAA1 & 354514 & 910627 & $3 / 31 / 2004$ & 37.64 & $4 / 16 / 2008$ & 41.59 & -4.0 \\
\hline 13N03W15CDD1 & 354526 & 911749 & $3 / 31 / 2004$ & 15.18 & $4 / 16 / 2008$ & 7.41 & 7.8 \\
\hline 13N03W36ABB1 & 354337 & 911532 & $4 / 06 / 2004$ & 14.2 & $3 / 27 / 2008$ & 16.3 & -2.1 \\
\hline 14N01W08AAA1 & 355216 & 910623 & $4 / 05 / 2004$ & 33.7 & $3 / 27 / 2008$ & 37.5 & -3.8 \\
\hline 14N01W09AAA1 & 355220 & 910515 & $3 / 31 / 2004$ & 40.32 & $4 / 16 / 2008$ & 43.88 & -3.6 \\
\hline 14N02W22BBC1 & 355026 & 911145 & $4 / 05 / 2004$ & 26 & $3 / 27 / 2008$ & 25.2 & 0.8 \\
\hline \multicolumn{8}{|c|}{ Jefferson County } \\
\hline 03S08W24BBC1 & 342620 & 914953 & $3 / 03 / 2004$ & 49.58 & $3 / 18 / 2008$ & 51.03 & -1.5 \\
\hline 03S09W06DDA1 & 342840 & 920037 & $3 / 03 / 2004$ & 35.61 & $3 / 18 / 2008$ & 37.02 & -1.4 \\
\hline 03S09W14BCD1 & 342712 & 915713 & $5 / 11 / 2004$ & 46.6 & $3 / 31 / 2008$ & 51 & -4.4 \\
\hline 03S09W22AAA1 & 342640 & 915728 & $5 / 11 / 2004$ & 43 & $3 / 31 / 2008$ & 43 & 0.0 \\
\hline 03S09W29CBD1 & 342517 & 920023 & $3 / 03 / 2004$ & 27.04 & $3 / 18 / 2008$ & 27.74 & -0.7 \\
\hline 03S09W36ACC1 & 342428 & 915555 & $5 / 20 / 2004$ & 46.3 & $3 / 31 / 2008$ & 29 & 17.3 \\
\hline 04S07W35DDB1 & 341836 & 914347 & $5 / 20 / 2004$ & 26.2 & $3 / 31 / 2008$ & 28.8 & -2.6 \\
\hline 04S08W13DCB1 & 342123 & 914926 & $3 / 03 / 2004$ & 46.12 & $3 / 18 / 2008$ & 47.99 & -1.9 \\
\hline 04S09W02CBD1 & 342325 & 915717 & $5 / 11 / 2004$ & 44.7 & $3 / 31 / 2008$ & 26.6 & 18.1 \\
\hline 04S09W32DDA1 & 341859 & 920009 & $5 / 11 / 2004$ & 19.7 & $3 / 31 / 2008$ & 16 & 3.7 \\
\hline 05S06W31CAA1 & 341330 & 914206 & $3 / 03 / 2004$ & 16.17 & $3 / 18 / 2008$ & 18.33 & -2.2 \\
\hline 05S07W29DDD1 & 341411 & 914654 & $5 / 20 / 2004$ & 18 & $3 / 31 / 2008$ & 16.1 & 1.9 \\
\hline 05S08W12DAA1 & 341712 & 914907 & $3 / 03 / 2004$ & 17.47 & $3 / 18 / 2008$ & 16.15 & 1.3 \\
\hline 06S05W15BCA1 & 341023 & 913245 & $3 / 03 / 2004$ & 19.44 & $3 / 18 / 2008$ & 18.22 & 1.2 \\
\hline 06S06W23AAD1 & 341007 & 913712 & $3 / 03 / 2004$ & 21.46 & $3 / 18 / 2008$ & 19.02 & 2.4 \\
\hline 06S07W14BAA1 & 341125 & 914426 & $3 / 03 / 2004$ & 16.23 & $3 / 18 / 2008$ & 15.25 & 1.0 \\
\hline 07S08W06BAA1 & 340859 & 915647 & $3 / 03 / 2004$ & 19.27 & $3 / 18 / 2008$ & 19.07 & 0.2 \\
\hline \multicolumn{8}{|c|}{ Lawrence County } \\
\hline 15N01W35CBB1 & 355336 & 910356 & $3 / 31 / 2004$ & 43.39 & $4 / 10 / 2008$ & 46.68 & -3.3 \\
\hline 16N01E11DAC2 & 360203 & 905639 & $3 / 31 / 2004$ & 43.66 & $4 / 10 / 2008$ & 48.69 & -5.0 \\
\hline 16N01W30DDC1 & 355937 & 910723 & $4 / 07 / 2004$ & 22 & $3 / 24 / 2008$ & 13 & 9.0 \\
\hline 16N02E34CBB1 & 355831 & 905208 & $4 / 07 / 2004$ & 43 & $3 / 24 / 2008$ & 50 & -7.0 \\
\hline 17N01E02BBA1 & 360901 & 905707 & $4 / 08 / 2004$ & 12.2 & $3 / 24 / 2008$ & 16.2 & -4.0 \\
\hline 17N02E04DCA1 & 360758 & 905224 & $4 / 06 / 2004$ & 38 & $3 / 24 / 2008$ & 44.2 & -6.2 \\
\hline
\end{tabular}


Appendix 2. Information pertaining to water levels measured in 2004 and 2008 in the Mississippi River Valley alluvial aquifer in eastern Arkansas.-Continued

[USGS, U.S. Geological Survey; NRCS, Natural Resources Conservation Service, ANRC, Arkansas Natural Resources Commission; Horizontal coordinate information is referenced to the North American Datum of 1983 (NAD83); *, control wells for duplicate measurements by USGS and NRCS for quality assurance]

\begin{tabular}{|c|c|c|c|c|c|c|c|}
\hline Station name & $\begin{array}{c}\text { Latitude } \\
\text { (degrees, } \\
\text { minutes, } \\
\text { seconds) }\end{array}$ & $\begin{array}{c}\text { Longitude } \\
\text { (degrees, } \\
\text { minutes, } \\
\text { seconds) }\end{array}$ & $\begin{array}{l}2004 \text { water- } \\
\text { level date }\end{array}$ & $\begin{array}{c}2004 \text { depth } \\
\text { to water } \\
\text { (feet below } \\
\text { land-surface } \\
\text { datum) }\end{array}$ & $\begin{array}{l}2008 \text { water- } \\
\text { level date }\end{array}$ & $\begin{array}{l}2008 \text { depth } \\
\text { to water } \\
\text { (feet below } \\
\text { land-surface } \\
\text { datum) }\end{array}$ & $\begin{array}{c}\text { Water-level } \\
\text { difference } \\
2004 \text { to } 2008 \\
\text { (feet) }\end{array}$ \\
\hline \multicolumn{8}{|c|}{ Lawrence County-Continued } \\
\hline 17N02E19CDC1* & 360516 & 905449 & $3 / 31 / 2004$ & 38.23 & $4 / 10 / 2008$ & 41.07 & -2.8 \\
\hline 17N02E19CDC1* & 360516 & 905449 & $4 / 06 / 2004$ & 36.6 & $4 / 15 / 2008$ & 35.5 & 1.1 \\
\hline \multicolumn{8}{|c|}{ Lee County } \\
\hline 01N01E09CCC1 & 344215 & 910054 & $4 / 17 / 2004$ & 30.5 & $4 / 15 / 2008$ & 16.7 & 13.8 \\
\hline 01N01E24CBD1 & 344033 & 905729 & $4 / 12 / 2004$ & 16.3 & $4 / 15 / 2008$ & 31 & -14.7 \\
\hline 01N02E01ADD1 & 344330 & 905016 & $4 / 07 / 2004$ & 25 & $4 / 15 / 2008$ & 33 & -8.0 \\
\hline 01N02E11BAB1 & 344255 & 905208 & $4 / 07 / 2004$ & 26 & $4 / 15 / 2008$ & 34 & -8.0 \\
\hline 01N02E12ABB1 & 344254 & 905040 & $4 / 07 / 2004$ & 26 & $4 / 15 / 2008$ & 29.5 & -3.5 \\
\hline 01N02E22CBA1 & 344056 & 905318 & $4 / 12 / 2004$ & 28.5 & $4 / 15 / 2008$ & 11 & 17.5 \\
\hline 01N02E33CBB1 & 343858 & 905434 & $4 / 12 / 2004$ & 14 & $4 / 15 / 2008$ & 9 & 5.0 \\
\hline 01N03E02BBC1 & 344339 & 904601 & $3 / 22 / 2004$ & 47.04 & $4 / 01 / 2008$ & 53.68 & -6.6 \\
\hline 01N03E35BBA1 & 343923 & 904549 & $3 / 22 / 2004$ & 9.18 & $4 / 01 / 2008$ & 13.85 & -4.7 \\
\hline 02N01E21BAA1 & 344633 & 910005 & $4 / 07 / 2004$ & 33.3 & $4 / 15 / 2008$ & 38.3 & -5.0 \\
\hline 02N01E23BAA2 & 344632 & 905820 & $3 / 22 / 2004$ & 49.22 & $4 / 01 / 2008$ & 52.1 & -2.9 \\
\hline 02N01W12BAA1 & 344828 & 910330 & $3 / 22 / 2004$ & 42.07 & $4 / 01 / 2008$ & 45.65 & -3.6 \\
\hline 02N01W34DDC1 & 344410 & 910520 & $4 / 12 / 2004$ & 47 & $4 / 15 / 2008$ & 52 & -5.0 \\
\hline 02N02E08ADC1 & 344807 & 905339 & $3 / 22 / 2004$ & 43.93 & $4 / 01 / 2008$ & 45.92 & -2.0 \\
\hline 02N02E21ABC1 & 344622 & 905358 & $3 / 22 / 2004$ & 38.73 & $4 / 01 / 2008$ & 41.25 & -2.5 \\
\hline 02N02E22BBB1 & 344628 & 905327 & $4 / 12 / 2004$ & 34 & $4 / 15 / 2008$ & 34 & 0.0 \\
\hline 02N03E08AAD1 & 344811 & 904838 & $3 / 22 / 2004$ & 43.43 & $4 / 01 / 2008$ & 45.73 & -2.3 \\
\hline 02N03E09DDD1 & 344723 & 904707 & $4 / 15 / 2004$ & 50 & $4 / 15 / 2008$ & 47 & 3.0 \\
\hline 02N03E29CAD1 & 344500 & 904846 & $4 / 15 / 2004$ & 38.5 & $4 / 15 / 2008$ & 43 & -4.5 \\
\hline 02N04E03ABD1 & 344855 & 903954 & $4 / 15 / 2004$ & 28.5 & $4 / 15 / 2008$ & 26 & 2.5 \\
\hline 02N04E15DAC1 & 344637 & 903950 & $3 / 22 / 2004$ & 18.15 & $4 / 01 / 2008$ & 20.67 & -2.5 \\
\hline 03N01E16CBA1 & 345222 & 910040 & $3 / 22 / 2004$ & 63.2 & $4 / 01 / 2008$ & 65.14 & -1.9 \\
\hline 03N02E12CDC1 & 345239 & 905053 & $4 / 12 / 2004$ & 27 & $4 / 15 / 2008$ & 43 & -16.0 \\
\hline 03N02E13BBA1 & 345237 & 905107 & $3 / 22 / 2004$ & 48.55 & $4 / 01 / 2008$ & 51.87 & -3.3 \\
\hline 03N02E21CBC1 & 345111 & 905428 & $4 / 12 / 2004$ & 55 & $4 / 15 / 2008$ & 56 & -1.0 \\
\hline 03N02E29DAD1 & 345014 & 905430 & $3 / 22 / 2004$ & 42.66 & $4 / 01 / 2008$ & 44.64 & -2.0 \\
\hline 03N03E05CDD1 & 345327 & 904837 & $4 / 15 / 2004$ & 32.5 & $4 / 15 / 2008$ & 49 & -16.5 \\
\hline 03N03E32CAB1 & 344933 & 904926 & $3 / 22 / 2004$ & 48.35 & $4 / 01 / 2008$ & 51.22 & -2.9 \\
\hline 03N04E07CBB1 & 345245 & 904312 & $4 / 15 / 2004$ & 32 & $4 / 15 / 2008$ & 30 & 2.0 \\
\hline 03N05E14DDA1 & 345148 & 903203 & $3 / 22 / 2004$ & 13.55 & $4 / 01 / 2008$ & 15.79 & -2.2 \\
\hline
\end{tabular}


Appendix 2. Information pertaining to water levels measured in 2004 and 2008 in the Mississippi River Valley alluvial aquifer in eastern Arkansas.-Continued

[USGS, U.S. Geological Survey; NRCS, Natural Resources Conservation Service, ANRC, Arkansas Natural Resources Commission; Horizontal coordinate information is referenced to the North American Datum of 1983 (NAD83); *, control wells for duplicate measurements by USGS and NRCS for quality assurance]

\begin{tabular}{|c|c|c|c|c|c|c|c|}
\hline Station name & $\begin{array}{l}\text { Latitude } \\
\text { (degrees, } \\
\text { minutes, } \\
\text { seconds) }\end{array}$ & $\begin{array}{l}\text { Longitude } \\
\text { (degrees, } \\
\text { minutes, } \\
\text { seconds) }\end{array}$ & $\begin{array}{l}2004 \text { water- } \\
\text { level date }\end{array}$ & $\begin{array}{c}2004 \text { depth } \\
\text { to water } \\
\text { (feet below } \\
\text { land-surface } \\
\text { datum) }\end{array}$ & $\begin{array}{l}2008 \text { water- } \\
\text { level date }\end{array}$ & $\begin{array}{c}2008 \text { depth } \\
\text { to water } \\
\text { (feet below } \\
\text { land-surface } \\
\text { datum) }\end{array}$ & $\begin{array}{c}\text { Water-level } \\
\text { difference } \\
2004 \text { to } 2008 \\
\text { (feet) }\end{array}$ \\
\hline \multicolumn{8}{|c|}{ Lincoln County } \\
\hline 07S06W03CCA2 & 340828 & 914114 & $5 / 11 / 2004$ & 16 & $3 / 12 / 2008$ & 18 & -2.0 \\
\hline 07S07W36CBD1 & 340411 & 914529 & $5 / 11 / 2004$ & 38 & $3 / 12 / 2008$ & 41 & -3.0 \\
\hline 08S04W06ABD1 & 340341 & 913116 & $5 / 11 / 2004$ & 17 & $3 / 12 / 2008$ & 16 & 1.0 \\
\hline 08S04W08BBB2 & 340254 & 913101 & $3 / 02 / 2004$ & 21.23 & $3 / 13 / 2008$ & 23.23 & -2.0 \\
\hline 08S04W29ABC1 & 340021 & 913044 & $5 / 11 / 2004$ & 45 & $3 / 12 / 2008$ & 45 & 0.0 \\
\hline 08S04W31CBA1 & 335901 & 913150 & $3 / 02 / 2004$ & 32.8 & $3 / 13 / 2008$ & 35.11 & -2.3 \\
\hline 08S05W12AAD1 & 340246 & 913214 & $5 / 11 / 2004$ & 21 & $3 / 12 / 2008$ & 24 & -3.0 \\
\hline 08S05W21DCD1 & 340027 & 913533 & $5 / 11 / 2004$ & 35 & $3 / 12 / 2008$ & 30 & 5.0 \\
\hline 08S05W32DCC1 & 335840 & 913644 & $5 / 11 / 2004$ & 33 & $3 / 12 / 2008$ & 47 & -14.0 \\
\hline 08S06W02ACB1 & 340339 & 913958 & $3 / 02 / 2004$ & 42.64 & $3 / 13 / 2008$ & 43.49 & -0.9 \\
\hline 09S04W06CBB1 & 335721 & 913252 & $5 / 11 / 2004$ & 45 & $3 / 12 / 2008$ & 39 & 6.0 \\
\hline 09S05W17BCB1 & 335552 & 913820 & $3 / 03 / 2004$ & 41.77 & $3 / 13 / 2008$ & 43.17 & -1.4 \\
\hline 09S05W19CCC1 & 335428 & 913941 & $5 / 11 / 2004$ & 32 & $3 / 12 / 2008$ & 40 & -8.0 \\
\hline 09S06W04BCD1 & 335821 & 914346 & $3 / 02 / 2004$ & 38.38 & $3 / 13 / 2008$ & 42.58 & -4.2 \\
\hline 09S06W04BDD1 & 335759 & 914335 & $5 / 11 / 2004$ & 41 & $3 / 12 / 2008$ & 46 & -5.0 \\
\hline 09S06W23CDB1 & 335440 & 914136 & $3 / 02 / 2004$ & 30.17 & $3 / 13 / 2008$ & 30.92 & -0.8 \\
\hline 10S05W06DCC1 & 335155 & 913908 & $3 / 03 / 2004$ & 30.38 & $3 / 13 / 2008$ & 32.37 & -2.0 \\
\hline \multicolumn{8}{|c|}{ Lonoke County } \\
\hline 01N08W03DDA1 & 344411 & 915050 & $4 / 8 / 2004$ & 140.4 & $4 / 15 / 2008$ & 137 & 3.4 \\
\hline 01N09W07DAA1 & 344337 & 920030 & $4 / 08 / 2004$ & 48.8 & $4 / 15 / 2008$ & 51 & -2.2 \\
\hline 01N09W13DAB1 & 344235 & 915517 & $3 / 12 / 2004$ & 87.12 & $3 / 19 / 2008$ & 88.43 & -1.3 \\
\hline 01N10W15CDA1 & 344236 & 920415 & $4 / 08 / 2004$ & 26.8 & $4 / 15 / 2008$ & 31 & -4.2 \\
\hline 01S06W31ABB1 & 343459 & 914131 & $3 / 11 / 2004$ & 78.7 & $3 / 19 / 2008$ & 79.32 & -0.6 \\
\hline 01S06W32BBB1 & 343501 & 914056 & $4 / 08 / 2004$ & 86.9 & $4 / 15 / 2008$ & 78.5 & 8.4 \\
\hline 01S07W12ABA1 & 343834 & 914230 & $3 / 11 / 2004$ & 68.77 & $3 / 19 / 2008$ & 71.77 & -3.0 \\
\hline 01S08W24CDD1 & 343606 & 914912 & $3 / 11 / 2004$ & 83.93 & $3 / 19 / 2008$ & 83.34 & 0.6 \\
\hline 01S09W36CCC1* & 343435 & 915619 & $3 / 12 / 2004$ & 62.27 & $3 / 18 / 2008$ & 61.91 & 0.4 \\
\hline 01S09W36CCC1* & 343435 & 915619 & $4 / 08 / 2004$ & 61.6 & $4 / 15 / 2008$ & 64 & -2.4 \\
\hline 01S10W01ACB1 & 343927 & 920215 & $3 / 12 / 2004$ & 47.24 & $3 / 18 / 2008$ & 44.63 & 2.6 \\
\hline 02N07W07DAA1 & 344845 & 914707 & $4 / 08 / 2004$ & 131.5 & $4 / 15 / 2008$ & 134 & -2.5 \\
\hline 02N07W16BAB1 & 344815 & 914540 & $3 / 11 / 2004$ & 136.19 & $3 / 19 / 2008$ & 135.79 & 0.4 \\
\hline 02N08W16ABC1 & 344806 & 915114 & $3 / 11 / 2004$ & 119.89 & $3 / 19 / 2008$ & 124.87 & -5.0 \\
\hline 02N08W23CAB1 & 344659 & 915118 & $4 / 08 / 2004$ & 138 & $4 / 15 / 2008$ & 129 & 9.0 \\
\hline 02N09W02BDB1 & 344955 & 915841 & $3 / 11 / 2004$ & 123.87 & $3 / 19 / 2008$ & 126.74 & -2.9 \\
\hline
\end{tabular}


Appendix 2. Information pertaining to water levels measured in 2004 and 2008 in the Mississippi River Valley alluvial aquifer in eastern Arkansas.-Continued

[USGS, U.S. Geological Survey; NRCS, Natural Resources Conservation Service, ANRC, Arkansas Natural Resources Commission; Horizontal coordinate information is referenced to the North American Datum of 1983 (NAD83); *, control wells for duplicate measurements by USGS and NRCS for quality assurance]

\begin{tabular}{|c|c|c|c|c|c|c|c|}
\hline Station name & $\begin{array}{l}\text { Latitude } \\
\text { (degrees, } \\
\text { minutes, } \\
\text { seconds) }\end{array}$ & $\begin{array}{l}\text { Longitude } \\
\text { (degrees, } \\
\text { minutes, } \\
\text { seconds) }\end{array}$ & $\begin{array}{l}2004 \text { water- } \\
\text { level date }\end{array}$ & $\begin{array}{l}2004 \text { depth } \\
\text { to water } \\
\text { (feet below } \\
\text { land-surface } \\
\text { datum) }\end{array}$ & $\begin{array}{l}2008 \text { water- } \\
\text { level date }\end{array}$ & $\begin{array}{l}2008 \text { depth } \\
\text { to water } \\
\text { (feet below } \\
\text { land-surface } \\
\text { datum) }\end{array}$ & $\begin{array}{c}\text { Water-level } \\
\text { difference } \\
2004 \text { to } 2008 \\
\text { (feet) }\end{array}$ \\
\hline \multicolumn{8}{|c|}{ Lonoke County-Continued } \\
\hline 02N10W15ACC1 & 344807 & 920353 & $4 / 08 / 2004$ & 31 & $4 / 15 / 2008$ & 32 & -1.0 \\
\hline 02S07W05CDC1 & 343326 & 914715 & $4 / 08 / 2004$ & 73.5 & $4 / 15 / 2008$ & 70 & 3.5 \\
\hline 02S07W10CCB1 & 343246 & 914525 & $3 / 11 / 2004$ & 62.48 & $3 / 19 / 2008$ & 63.34 & -0.9 \\
\hline 02S08W13BBB1 & 343232 & 914935 & $3 / 11 / 2004$ & 58.45 & $3 / 19 / 2008$ & 57.67 & 0.8 \\
\hline 02S08W34DBB1 & 343003 & 915150 & $3 / 12 / 2004$ & 62.68 & $3 / 19 / 2008$ & 63.76 & -1.1 \\
\hline 02S09W30CDD1 & 343014 & 920116 & $3 / 12 / 2004$ & 37.88 & $3 / 18 / 2008$ & 38.88 & -1.0 \\
\hline 03N07W08BDB1 & 345407 & 914638 & $4 / 16 / 2004$ & 93.72 & $2 / 07 / 2008$ & 98.31 & -4.6 \\
\hline 03N07W15DBC2 & 345253 & 914417 & $3 / 11 / 2004$ & 80.5 & $3 / 19 / 2008$ & 83.53 & -3.0 \\
\hline 03N07W29ADA1 & 345129 & 914558 & $4 / 16 / 2004$ & 89.27 & $2 / 07 / 2008$ & 92.27 & -3.0 \\
\hline 03N07W29CDD1 & 345057 & 914632 & $4 / 08 / 2004$ & 99.8 & $4 / 15 / 2008$ & 98 & 1.8 \\
\hline 03N07W35CDC2 & 344957 & 914332 & $3 / 11 / 2004$ & 114.63 & $3 / 19 / 2008$ & 117.23 & -2.6 \\
\hline 03N08W03BAA1 & 345519 & 915054 & $4 / 15 / 2004$ & 90.6 & $2 / 07 / 2008$ & 95.69 & -5.1 \\
\hline 03N08W03CCC1 & 345430 & 915123 & $4 / 15 / 2004$ & 98.87 & $2 / 07 / 2008$ & 105.1 & -6.2 \\
\hline 03N08W05CCC1 & 345429 & 915323 & $4 / 15 / 2004$ & 78.96 & $2 / 07 / 2008$ & 81.03 & -2.1 \\
\hline 03N08W08ABA1 & 345427 & 915248 & $4 / 15 / 2004$ & 91.45 & $2 / 07 / 2008$ & 95.51 & -4.1 \\
\hline 03N08W10ACB1 & 345415 & 915053 & $4 / 15 / 2004$ & 88.43 & $2 / 07 / 2008$ & 92.62 & -4.2 \\
\hline 03N08W10ADD1 & 345401 & 915023 & $4 / 15 / 2004$ & 88.99 & $2 / 07 / 2008$ & 95.05 & -6.1 \\
\hline 03N08W11ABD1 & 345419 & 914936 & $4 / 15 / 2004$ & 100.54 & $2 / 07 / 2008$ & 106.11 & -5.6 \\
\hline 03N08W11ACA1 & 345413 & 914934 & $4 / 14 / 2004$ & 98.5 & $2 / 07 / 2008$ & 104.23 & -5.7 \\
\hline 03N08W21BCC1 & 345220 & 915220 & $3 / 11 / 2004$ & 80.27 & $3 / 19 / 2008$ & 82.79 & -2.5 \\
\hline 03N08W29BBB1 & 345147 & 915333 & $4 / 15 / 2004$ & 110.26 & $2 / 07 / 2008$ & 113.34 & -3.1 \\
\hline 03N08W29BCC1 & 345125 & 915333 & $4 / 15 / 2004$ & 121.06 & $2 / 07 / 2008$ & 133.08 & -12.0 \\
\hline 03N08W32ABB1 & 345057 & 915257 & $4 / 14 / 2004$ & 116.75 & $2 / 07 / 2008$ & 120.61 & -3.9 \\
\hline 03N08W32ABB2 & 345057 & 915259 & $3 / 11 / 2004$ & 117.24 & $3 / 19 / 2008$ & 119.68 & -2.4 \\
\hline 03N08W34ADD1 & 345035 & 915028 & $4 / 15 / 2004$ & 121.55 & $2 / 07 / 2008$ & 121.67 & -0.1 \\
\hline 04N08W05ACA1 & 350020 & 915247 & $4 / 16 / 2004$ & 44.12 & $2 / 08 / 2008$ & 46.96 & -2.8 \\
\hline 04N08W10BDD1 & 345917 & 915055 & $4 / 16 / 2004$ & 27.14 & $2 / 08 / 2008$ & 30.47 & -3.3 \\
\hline 04N08W15BCB2 & 345833 & 915121 & $3 / 11 / 2004$ & 33.54 & $3 / 19 / 2008$ & 29.2 & 4.3 \\
\hline 04N08W16DCC1 & 345757 & 915154 & $4 / 16 / 2004$ & 45.12 & $2 / 07 / 2008$ & 47.88 & -2.8 \\
\hline 04N08W26AAD1 & 345652 & 914917 & $4 / 15 / 2004$ & 70.16 & $2 / 08 / 2008$ & 71.18 & -1.0 \\
\hline 04N08W28CAC1 & 345620 & 915216 & $4 / 16 / 2004$ & 53.27 & $2 / 07 / 2008$ & 55.9 & -2.6 \\
\hline 04N08W28CAD1 & 345626 & 915204 & $4 / 16 / 2004$ & 68.57 & $2 / 07 / 2008$ & 71.23 & -2.7 \\
\hline 04N08W28CCC1 & 345615 & 915225 & $4 / 15 / 2004$ & 59.09 & $2 / 07 / 2008$ & 61.43 & -2.3 \\
\hline 04N08W36DBB1 & 345541 & 914914 & $4 / 15 / 2004$ & 90.68 & $2 / 08 / 2008$ & 93.08 & -2.4 \\
\hline
\end{tabular}


Appendix 2. Information pertaining to water levels measured in 2004 and 2008 in the Mississippi River Valley alluvial aquifer in eastern Arkansas.-Continued

[USGS, U.S. Geological Survey; NRCS, Natural Resources Conservation Service, ANRC, Arkansas Natural Resources Commission; Horizontal coordinate information is referenced to the North American Datum of 1983 (NAD83); *, control wells for duplicate measurements by USGS and NRCS for quality assurance]

\begin{tabular}{|c|c|c|c|c|c|c|c|}
\hline Station name & $\begin{array}{l}\text { Latitude } \\
\text { (degrees, } \\
\text { minutes, } \\
\text { seconds) }\end{array}$ & $\begin{array}{l}\text { Longitude } \\
\text { (degrees, } \\
\text { minutes, } \\
\text { seconds) }\end{array}$ & $\begin{array}{l}2004 \text { water- } \\
\text { level date }\end{array}$ & $\begin{array}{l}2004 \text { depth } \\
\text { to water } \\
\text { (feet below } \\
\text { land-surface } \\
\text { datum) }\end{array}$ & $\begin{array}{l}2008 \text { water- } \\
\text { level date }\end{array}$ & $\begin{array}{l}2008 \text { depth } \\
\text { to water } \\
\text { (feet below } \\
\text { land-surface } \\
\text { datum) }\end{array}$ & $\begin{array}{c}\text { Water-level } \\
\text { difference } \\
2004 \text { to } 2008 \\
\text { (feet) }\end{array}$ \\
\hline \multicolumn{8}{|c|}{ Mississippi County } \\
\hline 10N08E21ABA1 & 352852 & 901415 & $4 / 15 / 2004$ & 25 & $4 / 17 / 2008$ & 25 & 0.0 \\
\hline 10N08E21BDC1 & 352830 & 901407 & $4 / 15 / 2004$ & 25 & $4 / 17 / 2008$ & 25.5 & -0.5 \\
\hline 10N08E22ABA2 & 352851 & 901312 & $3 / 24 / 2004$ & 22.34 & $4 / 07 / 2008$ & 25.53 & -3.2 \\
\hline 10N09E08ACC1 & 352949 & 900926 & $3 / 24 / 2004$ & 13.79 & $4 / 07 / 2008$ & 17.78 & -4.0 \\
\hline 11N09E34BBB1 & 353218 & 900715 & $3 / 24 / 2004$ & 14.78 & $4 / 07 / 2008$ & 19.12 & -4.3 \\
\hline 11N10E09BCB1 & 353530 & 900202 & $4 / 15 / 2004$ & 17 & $4 / 17 / 2008$ & 16 & 1.0 \\
\hline 12N08E08BCB1 & 354047 & 901559 & $3 / 24 / 2004$ & 8.32 & $4 / 07 / 2008$ & 6.26 & 2.1 \\
\hline 12N08E28DDB1 & 353707 & 901406 & $4 / 15 / 2004$ & 14.4 & $4 / 17 / 2008$ & 15.5 & -1.1 \\
\hline $12 \mathrm{~N} 09 \mathrm{E} 12 \mathrm{ABC} 1$ & 354054 & 900449 & $4 / 15 / 2004$ & 7.3 & $4 / 25 / 2008$ & 9 & -1.7 \\
\hline 12N10E04CAA1 & 354124 & 900136 & $4 / 15 / 2004$ & 8 & $4 / 17 / 2008$ & 8 & 0.0 \\
\hline 12N10E07BCD1 & 354036 & 900404 & $4 / 15 / 2004$ & 11 & $4 / 25 / 2008$ & 17 & -6.0 \\
\hline 12N10E21DBA1 & 353842 & 900122 & $4 / 15 / 2004$ & 11.7 & $4 / 17 / 2008$ & 16.5 & -4.8 \\
\hline 13N08E24ABB1 & 354428 & 901112 & $4 / 14 / 2004$ & 16.8 & $4 / 17 / 2008$ & 7.5 & 9.3 \\
\hline 13N09E30CCD1 & 354248 & 901029 & $3 / 24 / 2004$ & 8.95 & $4 / 07 / 2008$ & 7.08 & 1.9 \\
\hline 13N10E34DBB1 & 354218 & 900024 & $3 / 24 / 2004$ & 6.84 & $4 / 07 / 2008$ & 10.8 & -4.0 \\
\hline 14N08E12DAB1 & 355104 & 901052 & $3 / 24 / 2004$ & 5.33 & $4 / 07 / 2008$ & 2.75 & 2.6 \\
\hline 14N08E20DAA1 & 354921 & 901458 & $4 / 14 / 2004$ & 10 & $4 / 25 / 2008$ & 2.5 & 7.5 \\
\hline 14N08E26CC1 & 354803 & 901235 & $4 / 14 / 2004$ & 4 & $4 / 25 / 2008$ & 3.5 & 0.5 \\
\hline $14 \mathrm{~N} 10 \mathrm{E} 18 \mathrm{ABC} 1$ & 355022 & 900345 & $3 / 24 / 2004$ & 10.9 & $4 / 07 / 2008$ & 8.11 & 2.8 \\
\hline 14N11E03BCB1 & 355158 & 895433 & $3 / 25 / 2004$ & 3.36 & $4 / 07 / 2008$ & 3.52 & -0.2 \\
\hline 14N11E17CCB1 & 354955 & 895639 & $4 / 14 / 2004$ & 10 & $4 / 25 / 2008$ & 3 & 7.0 \\
\hline 14N11E33CAA1 & 354727 & 895508 & $4 / 14 / 2004$ & 12 & $3 / 25 / 2008$ & 10 & 2.0 \\
\hline 15N08E08DBC2 & 355605 & 901526 & $3 / 24 / 2004$ & 10.5 & $4 / 07 / 2008$ & 10.53 & 0.0 \\
\hline $15 \mathrm{~N} 10 \mathrm{E} 21 \mathrm{ABC} 1$ & 355447 & 900135 & $4 / 14 / 2004$ & 12 & $3 / 25 / 2008$ & 7.5 & 4.5 \\
\hline 15N12E01BCD1 & 355704 & 894601 & $4 / 14 / 2004$ & 7 & $3 / 25 / 2008$ & 9 & -2.0 \\
\hline 16N10E28BBD1* & 355906 & 900156 & $3 / 25 / 2004$ & 7.56 & $4 / 07 / 2008$ & 5.33 & 2.2 \\
\hline 16N10E28BBD1* & 355906 & 900156 & $4 / 14 / 2004$ & 12.5 & $3 / 25 / 2008$ & 5.5 & 7.0 \\
\hline 16N11E23ADA1 & 355947 & 895231 & $3 / 25 / 2004$ & 12.31 & $4 / 07 / 2008$ & 9.2 & 3.1 \\
\hline \multicolumn{8}{|c|}{ Monroe County } \\
\hline 01N01W21CDC2 & 344037 & 910707 & $3 / 15 / 2004$ & 34.47 & $3 / 27 / 2008$ & 39.68 & -5.2 \\
\hline 01N02W12CBC1 & 344242 & 911032 & $3 / 15 / 2004$ & 37.39 & $3 / 27 / 2008$ & 40.9 & -3.5 \\
\hline 01N03W23BAC1 & 344124 & 911743 & $4 / 14 / 2004$ & 14 & $3 / 20 / 2008$ & 14.3 & -0.3 \\
\hline 01N03W24BBB1 & 344135 & 911651 & $3 / 15 / 2004$ & 27.34 & $3 / 27 / 2008$ & 28.23 & -0.9 \\
\hline 01N04W33BBB2 & 343960 & 912649 & $3 / 15 / 2004$ & 94.28 & $3 / 27 / 2008$ & 97.56 & -3.3 \\
\hline
\end{tabular}


Appendix 2. Information pertaining to water levels measured in 2004 and 2008 in the Mississippi River Valley alluvial aquifer in eastern Arkansas.-Continued

[USGS, U.S. Geological Survey; NRCS, Natural Resources Conservation Service, ANRC, Arkansas Natural Resources Commission; Horizontal coordinate information is referenced to the North American Datum of 1983 (NAD83); *, control wells for duplicate measurements by USGS and NRCS for quality assurance]

\begin{tabular}{|c|c|c|c|c|c|c|c|}
\hline Station name & $\begin{array}{l}\text { Latitude } \\
\text { (degrees, } \\
\text { minutes, } \\
\text { seconds) }\end{array}$ & $\begin{array}{l}\text { Longitude } \\
\text { (degrees, } \\
\text { minutes, } \\
\text { seconds) }\end{array}$ & $\begin{array}{l}2004 \text { water- } \\
\text { level date }\end{array}$ & $\begin{array}{l}2004 \text { depth } \\
\text { to water } \\
\text { (feet below } \\
\text { land-surface } \\
\text { datum) }\end{array}$ & $\begin{array}{l}2008 \text { water- } \\
\text { level date }\end{array}$ & $\begin{array}{l}2008 \text { depth } \\
\text { to water } \\
\text { (feet below } \\
\text { land-surface } \\
\text { datum) }\end{array}$ & $\begin{array}{c}\text { Water-level } \\
\text { difference } \\
2004 \text { to } 2008 \\
\text { (feet) }\end{array}$ \\
\hline \multicolumn{8}{|c|}{ Monroe County-Continued } \\
\hline 01S01W13CDD1 & 343611 & 910341 & $3 / 17 / 2004$ & 20.44 & $3 / 27 / 2008$ & 22.52 & -2.1 \\
\hline 01S01W16DB & 343615 & 910632 & $4 / 15 / 2004$ & 17 & $3 / 27 / 2008$ & 20 & -3.0 \\
\hline 01S01W18DCD1 & 343618 & 910849 & $3 / 17 / 2004$ & 22.64 & $3 / 27 / 2008$ & 24.52 & -1.9 \\
\hline 01S02W20BBB1* & 343613 & 911456 & $3 / 17 / 2004$ & 11.74 & $3 / 27 / 2008$ & 12.22 & -0.5 \\
\hline 01S02W20BBB1* & 343613 & 911456 & $4 / 14 / 2004$ & 12 & $3 / 27 / 2008$ & 12 & 0.0 \\
\hline 01S03W20BBA1 & 343538 & 912118 & $3 / 15 / 2004$ & 72.46 & $3 / 27 / 2008$ & 75.03 & -2.6 \\
\hline 01S04W01BAB1 & 343906 & 912317 & $3 / 15 / 2004$ & 76.34 & $3 / 27 / 2008$ & 76.88 & -0.5 \\
\hline 02N01W19ADD1 & 344624 & 910814 & $4 / 15 / 2004$ & 51 & $3 / 27 / 2008$ & 54 & -3.0 \\
\hline 02N01W19BBA1 & 344645 & 910912 & $3 / 15 / 2004$ & 52.56 & $3 / 27 / 2008$ & 54.78 & -2.2 \\
\hline 02N03W35BCA1 & 344455 & 911745 & $4 / 15 / 2004$ & 31 & $3 / 20 / 2008$ & 35 & -4.0 \\
\hline 02S01W01BCD1 & 343305 & 910408 & $4 / 14 / 2004$ & 19 & $3 / 27 / 2008$ & 22 & -3.0 \\
\hline 02S02W11DAC1 & 343209 & 911101 & $3 / 17 / 2004$ & 9.93 & $3 / 27 / 2008$ & 11.04 & -1.1 \\
\hline 03N01W20ABA1 & 345201 & 910723 & $3 / 15 / 2004$ & 47.35 & $3 / 27 / 2008$ & 48.61 & -1.3 \\
\hline 03N02W31ADC1 & 344958 & 911447 & $3 / 15 / 2004$ & 39.53 & $3 / 27 / 2008$ & 39.15 & 0.4 \\
\hline 03N03W36AAA1 & 345027 & 911547 & $3 / 15 / 2004$ & 20.3 & $3 / 27 / 2008$ & 23.06 & -2.8 \\
\hline 04N02W01BCC1 & 345929 & 911004 & $4 / 15 / 2004$ & 38 & $3 / 20 / 2008$ & 39 & -1.0 \\
\hline 04N02W05BBB1 & 345957 & 911311 & $4 / 15 / 2004$ & 15 & $3 / 20 / 2008$ & 16 & -1.0 \\
\hline 04N02W27CDD3 & 345540 & 911150 & $3 / 15 / 2004$ & 45.43 & $3 / 27 / 2008$ & 45.68 & -0.3 \\
\hline 04N02W28DDD3 & 345535 & 911221 & $3 / 15 / 2004$ & 33.04 & $3 / 27 / 2008$ & 32.81 & 0.2 \\
\hline 04N02W30BBB1 & 345628 & 911525 & $3 / 15 / 2004$ & 13.41 & $3 / 27 / 2008$ & 13.8 & -0.4 \\
\hline \multicolumn{8}{|c|}{ Phillips County } \\
\hline 01S01E20DDB1 & 343529 & 910058 & $4 / 12 / 2004$ & 25 & $4 / 14 / 2008$ & 27 & -2.0 \\
\hline 01S02E09CBB1* & 343719 & 905434 & $3 / 17 / 2004$ & 11.46 & $3 / 24 / 2008$ & 12.8 & -1.3 \\
\hline 01S02E09CBB1* & 343719 & 905434 & $4 / 12 / 2004$ & 9 & $4 / 01 / 2008$ & 15.44 & -6.4 \\
\hline 01S03E02ADD1 & 343814 & 904511 & $4 / 12 / 2004$ & 13 & $3 / 24 / 2008$ & 17 & -4.0 \\
\hline 01S03E10ABB1 & 343741 & 904634 & $4 / 12 / 2004$ & 14 & $3 / 24 / 2008$ & 19 & -5.0 \\
\hline 01S04E05DCD1* & 343802 & 904151 & $3 / 17 / 2004$ & 45.18 & $4 / 01 / 2008$ & 50.23 & -5.1 \\
\hline 01S04E05DCD1* & 343802 & 904151 & $4 / 12 / 2004$ & 45 & $3 / 24 / 2008$ & 49 & -4.0 \\
\hline 02S01E28CCB1 & 342916 & 910058 & $3 / 17 / 2004$ & 17.56 & $3 / 31 / 2008$ & 18.63 & -1.1 \\
\hline 02S02E29DDD1 & 342901 & 905444 & $4 / 12 / 2004$ & 25.6 & $4 / 14 / 2008$ & 24 & 1.6 \\
\hline 02S02E33ACC1 & 342824 & 905412 & $4 / 12 / 2004$ & 25 & $4 / 14 / 2008$ & 24 & 1.0 \\
\hline 02S03E15ACD1 & 343110 & 904621 & $3 / 17 / 2004$ & 10.6 & $5 / 12 / 2008$ & 8.59 & 2.0 \\
\hline 02S03E34BCD1 & 342828 & 904653 & $4 / 13 / 2004$ & 17.8 & $3 / 25 / 2008$ & 20.4 & -2.6 \\
\hline 02S04E27AAC1* & 342932 & 904001 & $3 / 17 / 2004$ & 3.93 & $3 / 31 / 2008$ & 9.74 & -5.8 \\
\hline
\end{tabular}


Appendix 2. Information pertaining to water levels measured in 2004 and 2008 in the Mississippi River Valley alluvial aquifer in eastern Arkansas.-Continued

[USGS, U.S. Geological Survey; NRCS, Natural Resources Conservation Service, ANRC, Arkansas Natural Resources Commission; Horizontal coordinate information is referenced to the North American Datum of 1983 (NAD83); *, control wells for duplicate measurements by USGS and NRCS for quality assurance]

\begin{tabular}{|c|c|c|c|c|c|c|c|}
\hline Station name & $\begin{array}{l}\text { Latitude } \\
\text { (degrees, } \\
\text { minutes, } \\
\text { seconds) }\end{array}$ & $\begin{array}{l}\text { Longitude } \\
\text { (degrees, } \\
\text { minutes, } \\
\text { seconds) }\end{array}$ & $\begin{array}{l}2004 \text { water- } \\
\text { level date }\end{array}$ & $\begin{array}{c}2004 \text { depth } \\
\text { to water } \\
\text { (feet below } \\
\text { land-surface } \\
\text { datum) }\end{array}$ & $\begin{array}{l}2008 \text { water- } \\
\text { level date }\end{array}$ & $\begin{array}{c}2008 \text { depth } \\
\text { to water } \\
\text { (feet below } \\
\text { land-surface } \\
\text { datum) }\end{array}$ & $\begin{array}{c}\text { Water-level } \\
\text { difference } \\
2004 \text { to } 2008 \\
\text { (feet) }\end{array}$ \\
\hline \multicolumn{8}{|c|}{ Phillips County-Continued } \\
\hline 02S04E27AAC1* & 342932 & 904001 & $4 / 13 / 2004$ & 6.2 & $3 / 25 / 2008$ & 7 & -0.8 \\
\hline 03S02E35DDA1 & 342256 & 905130 & $3 / 17 / 2004$ & 19.9 & $3 / 31 / 2008$ & 22.44 & -2.5 \\
\hline 03S03E04DAA1 & 342735 & 904710 & $3 / 17 / 2004$ & 18.27 & $3 / 31 / 2008$ & 20.37 & -2.1 \\
\hline 03S04E02CAA1* & 342732 & 903918 & $3 / 17 / 2004$ & 8.23 & $3 / 31 / 2008$ & 17.48 & -9.3 \\
\hline 03S04E02CAA1* & 342732 & 903918 & $4 / 13 / 2004$ & 11.8 & $3 / 25 / 2008$ & 15.5 & -3.7 \\
\hline 04S01E01AAD1 & 342238 & 905700 & $4 / 13 / 2004$ & 14.4 & $3 / 25 / 2008$ & 18 & -3.6 \\
\hline 04S01E14CDD1 & 342014 & 905837 & $4 / 13 / 2004$ & 12 & $3 / 25 / 2008$ & 16 & -4.0 \\
\hline 04S01E23CCA1 & 341931 & 905853 & $3 / 17 / 2004$ & 11.96 & $3 / 31 / 2008$ & 14.14 & -2.2 \\
\hline 04S01E29CDC1 & 341844 & 910148 & $4 / 13 / 2004$ & 7.9 & $3 / 25 / 2008$ & 7 & 0.9 \\
\hline 04S02E01DBB1 & 342220 & 905053 & $4 / 13 / 2004$ & 9.6 & $3 / 25 / 2008$ & 16 & -6.4 \\
\hline 05S02E18BDA1 & 341535 & 905628 & $3 / 17 / 2004$ & 16.14 & $3 / 31 / 2008$ & 23.53 & -7.4 \\
\hline \multicolumn{8}{|c|}{ Poinsett County } \\
\hline 10N01E02AAA & 353205 & 905654 & $3 / 19 / 2004$ & 97 & $4 / 07 / 2008$ & 101 & -4.0 \\
\hline 10N01E14CC1 & 352910 & 905814 & $3 / 29 / 2004$ & 89.72 & $4 / 08 / 2008$ & 94.91 & -5.2 \\
\hline 10N01E16CCB1 & 352922 & 910005 & $3 / 29 / 2004$ & 72.39 & $4 / 08 / 2008$ & 77.67 & -5.3 \\
\hline 10N01E32CBB1 & 352657 & 910053 & $3 / 19 / 2004$ & 70 & $3 / 26 / 2008$ & 76.5 & -6.5 \\
\hline 10N01E33ACB1 & 352746 & 905931 & $3 / 19 / 2004$ & 76 & $3 / 26 / 2008$ & 81 & -5.0 \\
\hline 10N02E13BCC1 & 352949 & 905026 & $3 / 29 / 2004$ & 99.9 & $4 / 08 / 2008$ & 105.24 & -5.3 \\
\hline 10N02E20BAB1 & 352906 & 905418 & $3 / 19 / 2004$ & 104 & $3 / 26 / 2008$ & 106 & -2.0 \\
\hline 10N03E14DAB1 & 352947 & 904405 & $3 / 29 / 2004$ & 116.95 & $4 / 08 / 2008$ & 119.6 & -2.7 \\
\hline 10N03E35CDD1 & 352656 & 904436 & $3 / 29 / 2004$ & 123.86 & $4 / 08 / 2008$ & 125.48 & -1.6 \\
\hline 10N04E35BBA1 & 352745 & 903831 & $3 / 17 / 2004$ & 20 & $3 / 24 / 2008$ & 19.5 & 0.5 \\
\hline 10N05E15BDD1 & 352937 & 903253 & $3 / 29 / 2004$ & 12.17 & $4 / 08 / 2008$ & 12.71 & -0.5 \\
\hline 10N07E22AAC1 & 352847 & 901935 & $3 / 29 / 2004$ & 27.56 & $4 / 08 / 2008$ & 28.86 & -1.3 \\
\hline 11N01E17DDC1 & 353437 & 910015 & $3 / 19 / 2004$ & 77 & $4 / 07 / 2008$ & 81 & -4.0 \\
\hline 11N01E17DDD1 & 353437 & 910013 & $3 / 26 / 2004$ & 76.12 & $4 / 08 / 2008$ & 80.89 & -4.8 \\
\hline 11N01E26AA1 & 353340 & 905653 & $3 / 26 / 2004$ & 91.95 & $4 / 08 / 2008$ & 96.63 & -4.7 \\
\hline 11N01E34AAA & 353256 & 905759 & $3 / 19 / 2004$ & 85 & $4 / 07 / 2008$ & 90.5 & -5.5 \\
\hline 11N02E26AAB1 & 353350 & 905034 & $3 / 29 / 2004$ & 105.42 & $4 / 08 / 2008$ & 110.11 & -4.7 \\
\hline 11N02E30BBB1 & 353352 & 905540 & $3 / 19 / 2004$ & 100 & $4 / 07 / 2008$ & 105 & -5.0 \\
\hline 11N02E34CBA1 & 353238 & 905222 & $3 / 19 / 2004$ & 105 & $4 / 07 / 2008$ & 110 & -5.0 \\
\hline 11N03E10DDA1 & 353546 & 904457 & $3 / 29 / 2004$ & 102.99 & $4 / 08 / 2008$ & 106.11 & -3.1 \\
\hline 11N03E18BAB1 & 353538 & 904852 & $3 / 29 / 2004$ & 103.23 & $4 / 08 / 2008$ & 106.53 & -3.3 \\
\hline 11N04E36ABA1 & 353251 & 903654 & $3 / 17 / 2004$ & 16 & $3 / 24 / 2008$ & 16.5 & -0.5 \\
\hline
\end{tabular}


Appendix 2. Information pertaining to water levels measured in 2004 and 2008 in the Mississippi River Valley alluvial aquifer in eastern Arkansas.-Continued

[USGS, U.S. Geological Survey; NRCS, Natural Resources Conservation Service, ANRC, Arkansas Natural Resources Commission; Horizontal coordinate information is referenced to the North American Datum of 1983 (NAD83); *, control wells for duplicate measurements by USGS and NRCS for quality assurance]

\begin{tabular}{|c|c|c|c|c|c|c|c|}
\hline Station name & $\begin{array}{c}\text { Latitude } \\
\text { (degrees, } \\
\text { minutes, } \\
\text { seconds) }\end{array}$ & $\begin{array}{l}\text { Longitude } \\
\text { (degrees, } \\
\text { minutes, } \\
\text { seconds) }\end{array}$ & $\begin{array}{l}2004 \text { water- } \\
\text { level date }\end{array}$ & $\begin{array}{c}2004 \text { depth } \\
\text { to water } \\
\text { (feet below } \\
\text { land-surface } \\
\text { datum) }\end{array}$ & $\begin{array}{l}2008 \text { water- } \\
\text { level date }\end{array}$ & $\begin{array}{c}2008 \text { depth } \\
\text { to water } \\
\text { (feet below } \\
\text { land-surface } \\
\text { datum) }\end{array}$ & $\begin{array}{c}\text { Water-level } \\
\text { difference } \\
2004 \text { to } 2008 \\
\text { (feet) }\end{array}$ \\
\hline \multicolumn{8}{|c|}{ Poinsett County-Continued } \\
\hline 11N07E18CAB1 & 353435 & 902320 & $3 / 29 / 2004$ & 13.89 & $4 / 08 / 2008$ & 14.04 & -0.2 \\
\hline 12N01E07CDA1 & 354054 & 910141 & $3 / 26 / 2004$ & 51.89 & $4 / 08 / 2008$ & 55.14 & -3.3 \\
\hline 12N01E22DAB1 & 353922 & 905809 & $3 / 19 / 2004$ & 73 & $4 / 07 / 2008$ & 76.5 & -3.5 \\
\hline 12N02E25DCC1 & 353820 & 904944 & $3 / 19 / 2004$ & 112 & $4 / 07 / 2008$ & 115 & -3.0 \\
\hline $12 \mathrm{~N} 02 \mathrm{E} 34 \mathrm{CCC} 1$ & 353724 & 905230 & $3 / 19 / 2004$ & 110 & $4 / 07 / 2008$ & 115.5 & -5.5 \\
\hline 12N03E01CBD1 & 354154 & 904329 & $3 / 19 / 2004$ & 92 & $3 / 26 / 2008$ & 96 & -4.0 \\
\hline 12N04E08CDA & 354053 & 904112 & $3 / 17 / 2004$ & 87 & $3 / 26 / 2008$ & 95 & -8.0 \\
\hline 12N05E16ABA1 & 354039 & 903333 & $3 / 17 / 2004$ & 9 & $3 / 26 / 2008$ & 9.5 & -0.5 \\
\hline 12N05E34ABA1 & 353805 & 903230 & $3 / 29 / 2004$ & 7.47 & $4 / 08 / 2008$ & 7.13 & 0.3 \\
\hline 12N07E04BAA1 & 354202 & 902060 & $3 / 29 / 2004$ & 8.15 & $4 / 08 / 2008$ & 2.42 & 5.7 \\
\hline 12N07E10CBB1 & 354042 & 902022 & $3 / 17 / 2004$ & 10 & $3 / 24 / 2008$ & 10 & 0.0 \\
\hline \multicolumn{8}{|c|}{ Prairie County } \\
\hline 01N06W05CCB1 & 344353 & 914049 & $3 / 10 / 2004$ & 117.1 & $3 / 26 / 2008$ & 119.27 & -2.2 \\
\hline 01N06W29DDD1 & 344018 & 913951 & $3 / 10 / 2004$ & 115.91 & $3 / 26 / 2008$ & 118.13 & -2.2 \\
\hline 01S04W28BBC1 & 343529 & 912650 & $4 / 19 / 2004$ & 121.6 & $4 / 02 / 2008$ & 100 & 21.6 \\
\hline 01S04W28BDB1 & 343523 & 912630 & $3 / 09 / 2004$ & 97.25 & $3 / 25 / 2008$ & 98 & -0.8 \\
\hline 01S05W14BBC1 & 343722 & 913109 & $3 / 09 / 2004$ & 107.74 & $3 / 25 / 2008$ & 109.68 & -1.9 \\
\hline 01S05W31DDA1 & 343417 & 913432 & $3 / 09 / 2004$ & 110.7 & $3 / 26 / 2008$ & 105.57 & 5.1 \\
\hline 02N04W02BCB1 & 344916 & 912419 & $3 / 10 / 2004$ & 19.13 & $3 / 25 / 2008$ & 19.88 & -0.8 \\
\hline 02N04W32CCB1 & 344436 & 912738 & $3 / 10 / 2004$ & 83.73 & $3 / 25 / 2008$ & 84.84 & -1.1 \\
\hline 02N05W06BAB1 & 344958 & 913421 & $3 / 10 / 2004$ & 88.79 & $3 / 26 / 2008$ & 89.81 & -1.0 \\
\hline 02N05W13AAB1 & 344805 & 912854 & $3 / 10 / 2004$ & 77.77 & $3 / 25 / 2008$ & 51.83 & 25.9 \\
\hline 02N05W29DDB2 & 344545 & 913309 & $3 / 10 / 2004$ & 118.24 & $3 / 26 / 2008$ & 119.78 & -1.5 \\
\hline 02N06W17ABB1 & 344809 & 913959 & $3 / 10 / 2004$ & 123.16 & $3 / 26 / 2008$ & 125.82 & -2.7 \\
\hline 02S06W14BBB1 & 343213 & 913729 & $3 / 09 / 2004$ & 62.25 & $3 / 26 / 2008$ & 75.76 & -13.5 \\
\hline 03N04W03AAC1 & 345439 & 912424 & $3 / 10 / 2004$ & 26.83 & $3 / 25 / 2008$ & 26.91 & -0.1 \\
\hline 03N05W03BDD2 & 345444 & 913115 & $3 / 10 / 2004$ & 66.27 & $3 / 26 / 2008$ & 65.25 & 1.0 \\
\hline 03N06W01BCB1 & 345455 & 913601 & $3 / 10 / 2004$ & 80.64 & $3 / 26 / 2008$ & 80.72 & -0.1 \\
\hline 03N06W19BDD1 & 345207 & 914110 & $3 / 10 / 2004$ & 85.24 & $3 / 26 / 2008$ & 86.33 & -1.1 \\
\hline 04N04W07ADC1 & 345850 & 912733 & $3 / 10 / 2004$ & 25.53 & $3 / 25 / 2008$ & 23.66 & 1.9 \\
\hline 04N05W07CDC1 & 345043 & 913441 & $3 / 10 / 2004$ & 74.42 & $3 / 25 / 2008$ & 79.34 & -4.9 \\
\hline 04N05W31DDC1 & 345514 & 913406 & $3 / 10 / 2004$ & 76.23 & $3 / 26 / 2008$ & 76.25 & 0.0 \\
\hline 04N06W05CCC1 & 345934 & 914018 & $3 / 10 / 2004$ & 59.96 & $3 / 25 / 2008$ & 80.49 & -20.5 \\
\hline 04N07W03DCB1 & 345942 & 914412 & $3 / 10 / 2004$ & 86.61 & $3 / 25 / 2008$ & 88.73 & -2.1 \\
\hline
\end{tabular}


Appendix 2. Information pertaining to water levels measured in 2004 and 2008 in the Mississippi River Valley alluvial aquifer in eastern Arkansas.-Continued

[USGS, U.S. Geological Survey; NRCS, Natural Resources Conservation Service, ANRC, Arkansas Natural Resources Commission; Horizontal coordinate information is referenced to the North American Datum of 1983 (NAD83); *, control wells for duplicate measurements by USGS and NRCS for quality assurance]

\begin{tabular}{|c|c|c|c|c|c|c|c|}
\hline Station name & $\begin{array}{l}\text { Latitude } \\
\text { (degrees, } \\
\text { minutes, } \\
\text { seconds) }\end{array}$ & $\begin{array}{l}\text { Longitude } \\
\text { (degrees, } \\
\text { minutes, } \\
\text { seconds) }\end{array}$ & $\begin{array}{c}2004 \text { water- } \\
\text { level date }\end{array}$ & $\begin{array}{c}2004 \text { depth } \\
\text { to water } \\
\text { (feet below } \\
\text { land-surface } \\
\text { datum) }\end{array}$ & $\begin{array}{l}2008 \text { water- } \\
\text { level date }\end{array}$ & $\begin{array}{c}2008 \text { depth } \\
\text { to water } \\
\text { (feet below } \\
\text { land-surface } \\
\text { datum) }\end{array}$ & $\begin{array}{c}\text { Water-level } \\
\text { difference } \\
2004 \text { to } 2008 \\
\text { (feet) }\end{array}$ \\
\hline \multicolumn{8}{|c|}{ Prairie County-Continued } \\
\hline 04N07W20DDB1 & 345709 & 914607 & $4 / 15 / 2004$ & 109.52 & $2 / 07 / 2008$ & 103.11 & 6.4 \\
\hline 04N07W28BBA1 & 345701 & 914545 & $3 / 10 / 2004$ & 94.75 & $3 / 25 / 2008$ & 97.67 & -2.9 \\
\hline 05N05W14DCD1 & 350252 & 913034 & $3 / 10 / 2004$ & 37.96 & $3 / 25 / 2008$ & 31.74 & 6.2 \\
\hline \multicolumn{8}{|c|}{ Pulaski County } \\
\hline 01S10W29CC1 & 343538 & 920708 & $3 / 19 / 2004$ & 16.65 & $3 / 18 / 2008$ & 14.87 & 1.8 \\
\hline 02S10W14DC1 & 343205 & 920334 & $3 / 19 / 2004$ & 25.57 & $3 / 18 / 2008$ & 23.5 & 2.1 \\
\hline 02S10W16CCA1 & 343217 & 920549 & $3 / 19 / 2004$ & 24.92 & $3 / 18 / 2008$ & 25.33 & -0.4 \\
\hline \multicolumn{8}{|c|}{ Randolph County } \\
\hline 18N01E13BAB1 & 361230 & 905551 & $4 / 23 / 2004$ & 15.7 & $4 / 08 / 2008$ & 16 & -0.3 \\
\hline 18N01E28AAD1 & 361040 & 905820 & $4 / 22 / 2004$ & 15.3 & $4 / 08 / 2008$ & 13.5 & 1.8 \\
\hline 18N01E34AAC1 & 360943 & 905729 & $3 / 30 / 2004$ & 15.93 & $4 / 10 / 2008$ & 15.9 & 0.0 \\
\hline 18N02E03DAD1 & 361336 & 905043 & $4 / 23 / 2004$ & 34.9 & $4 / 08 / 2008$ & 32.5 & 2.4 \\
\hline 18N02E17CBB1 & 361204 & 905356 & $4 / 22 / 2004$ & 16 & $4 / 08 / 2008$ & 19 & -3.0 \\
\hline 18N02E20BDA1 & 361125 & 905332 & $4 / 23 / 2004$ & 35.2 & $4 / 08 / 2008$ & 32.5 & 2.7 \\
\hline 18N02E22DCD1 & 361046 & 905105 & $3 / 30 / 2004$ & 36.27 & $4 / 10 / 2008$ & 39.12 & -2.9 \\
\hline 18N02E34BCC1 & 360933 & 905150 & $4 / 23 / 2004$ & 14.9 & $4 / 08 / 2008$ & 35.5 & -20.6 \\
\hline 19N02E09DCA1 & 361757 & 905157 & $3 / 30 / 2004$ & 10.12 & $4 / 10 / 2008$ & -0.5 & 10.6 \\
\hline 20N02E01ADD1 & 362424 & 904811 & $3 / 30 / 2004$ & 11.22 & $4 / 10 / 2008$ & 2.66 & 8.6 \\
\hline 20N02E01ADD2 & 362424 & 904811 & $4 / 23 / 2004$ & 13 & $4 / 10 / 2008$ & 3 & 10.0 \\
\hline 20N02E12BAA1 & 362352 & 904848 & $4 / 23 / 2004$ & 7 & $4 / 09 / 2008$ & 19 & -12.0 \\
\hline 20N02E14DAB1 & 362232 & 904930 & $4 / 23 / 2004$ & 8.6 & $4 / 10 / 2008$ & 18 & -9.4 \\
\hline 20N03E28BA1 & 362114 & 904538 & $3 / 30 / 2004$ & 10.93 & $4 / 10 / 2008$ & 9.43 & 1.5 \\
\hline 20N03E33CCA1 & 361941 & 904552 & $4 / 22 / 2004$ & 21.3 & $4 / 09 / 2008$ & 23.5 & -2.2 \\
\hline \multicolumn{8}{|c|}{ St. Francis County } \\
\hline 04N01E13ADA1 & 345755 & 905638 & $3 / 23 / 2004$ & 57.87 & $4 / 02 / 2008$ & 61.56 & -3.7 \\
\hline 04N01W28CDD1 & 345535 & 910634 & $3 / 23 / 2004$ & 68.28 & $4 / 02 / 2008$ & 72.51 & -4.2 \\
\hline 04N02E19BBB1 & 345701 & 905633 & $3 / 23 / 2004$ & 58.36 & $4 / 02 / 2008$ & 61.77 & -3.4 \\
\hline 04N03E21DAD1 & 345623 & 904655 & $3 / 23 / 2004$ & 56.96 & $4 / 02 / 2008$ & 60.37 & -3.4 \\
\hline 04N05E22BBB1 & 345651 & 903357 & $3 / 22 / 2004$ & 26.59 & $4 / 02 / 2008$ & 27.22 & -0.6 \\
\hline 05N01E15BCB1 & 350303 & 905942 & $3 / 23 / 2004$ & 63.48 & $4 / 02 / 2008$ & 69.66 & -6.2 \\
\hline 05N01E27BBA1 & 350136 & 905929 & $3 / 23 / 2004$ & 65.43 & $4 / 02 / 2008$ & 69.07 & -3.6 \\
\hline 05N02E20ADC1 & 350157 & 905437 & $3 / 23 / 2004$ & 53.08 & $4 / 02 / 2008$ & 56.07 & -3.0 \\
\hline 05N03E20AAA2 & 350214 & 904801 & $3 / 23 / 2004$ & 102.73 & $4 / 02 / 2008$ & 106.09 & -3.4 \\
\hline 05N06E34CAB1 & 350026 & 902657 & $3 / 22 / 2004$ & 26.46 & $4 / 02 / 2008$ & 28.38 & -1.9 \\
\hline
\end{tabular}


Appendix 2. Information pertaining to water levels measured in 2004 and 2008 in the Mississippi River Valley alluvial aquifer in eastern Arkansas.-Continued

[USGS, U.S. Geological Survey; NRCS, Natural Resources Conservation Service, ANRC, Arkansas Natural Resources Commission; Horizontal coordinate information is referenced to the North American Datum of 1983 (NAD83); *, control wells for duplicate measurements by USGS and NRCS for quality assurance]

\begin{tabular}{|c|c|c|c|c|c|c|c|}
\hline Station name & $\begin{array}{l}\text { Latitude } \\
\text { (degrees, } \\
\text { minutes, } \\
\text { seconds) }\end{array}$ & $\begin{array}{l}\text { Longitude } \\
\text { (degrees, } \\
\text { minutes, } \\
\text { seconds) }\end{array}$ & $\begin{array}{l}2004 \text { water- } \\
\text { level date }\end{array}$ & $\begin{array}{l}2004 \text { depth } \\
\text { to water } \\
\text { (feet below } \\
\text { land-surface } \\
\text { datum) }\end{array}$ & $\begin{array}{l}2008 \text { water- } \\
\text { level date }\end{array}$ & $\begin{array}{l}2008 \text { depth } \\
\text { to water } \\
\text { (feet below } \\
\text { land-surface } \\
\text { datum) }\end{array}$ & $\begin{array}{c}\text { Water-level } \\
\text { difference } \\
2004 \text { to } 2008 \\
\text { (feet) }\end{array}$ \\
\hline \multicolumn{8}{|c|}{ St. Francis County-Continued } \\
\hline 06N01E33ACA2 & 350552 & 905942 & $3 / 23 / 2004$ & 65.34 & $4 / 02 / 2008$ & 69.41 & -4.1 \\
\hline 06N02E13DCA1 & 350813 & 905003 & $3 / 23 / 2004$ & 73.61 & $4 / 02 / 2008$ & 75.8 & -2.2 \\
\hline 06N02E15BDD1 & 350842 & 905247 & $3 / 23 / 2004$ & 58.57 & $4 / 02 / 2008$ & 62.23 & -3.7 \\
\hline 06N02E24AAA1 & 350755 & 905002 & $3 / 23 / 2004$ & 71.49 & $4 / 02 / 2008$ & 72.94 & -1.5 \\
\hline 06N05E22ACC1 & 350723 & 903252 & $3 / 22 / 2004$ & 39.44 & $4 / 02 / 2008$ & 42.82 & -3.4 \\
\hline 06N06E20ABB2 & 350747 & 902841 & $3 / 22 / 2004$ & 35.25 & $4 / 02 / 2008$ & 37.3 & -2.1 \\
\hline \multicolumn{8}{|c|}{ White County } \\
\hline 05N07W09AAA1 & 350447 & 914441 & $3 / 18 / 2004$ & 14.82 & $4 / 16 / 2008$ & 12.62 & 2.2 \\
\hline 05N07W10CCC1 & 350400 & 914436 & $3 / 18 / 2004$ & 8.78 & $4 / 16 / 2008$ & 7.79 & 1.0 \\
\hline 06N06W04BAA1 & 351047 & 913910 & $3 / 18 / 2004$ & 36.45 & $4 / 16 / 2008$ & 28.9 & 7.6 \\
\hline 06N06W04BAD1 & 351037 & 913903 & $4 / 16 / 2004$ & 41.5 & $3 / 27 / 2008$ & 36.7 & 4.8 \\
\hline 06N06W13DBB1 & 350918 & 913552 & $4 / 16 / 2004$ & 46.9 & $3 / 27 / 2008$ & 45.8 & 1.1 \\
\hline 06N06W18BBC1 & 350851 & 914152 & $3 / 18 / 2004$ & 14.67 & $4 / 16 / 2008$ & 12.09 & 2.6 \\
\hline 06N06W18BCA1 & 350835 & 914150 & $4 / 16 / 2004$ & 24.2 & $3 / 27 / 2008$ & 13.8 & 10.4 \\
\hline 06N06W34AAB1 & 350624 & 913754 & $3 / 18 / 2004$ & 59.7 & $4 / 16 / 2008$ & 60.78 & -1.1 \\
\hline 06N07W17DCC1 & 350822 & 914635 & $3 / 18 / 2004$ & 12.21 & $4 / 16 / 2008$ & 13.42 & -1.2 \\
\hline 06N08W13ABA1 & 350908 & 914824 & $3 / 18 / 2004$ & 7.4 & $4 / 16 / 2008$ & 6.6 & 0.8 \\
\hline 06N08W26DDB1 & 350640 & 914931 & $3 / 18 / 2004$ & 12.25 & $4 / 16 / 2008$ & 13.23 & -1.0 \\
\hline 07N05W01AAA1 & 351553 & 912858 & $3 / 18 / 2004$ & 15.35 & $4 / 16 / 2008$ & 11.11 & 4.2 \\
\hline 07N05W32BAB1 & 351137 & 913406 & $3 / 18 / 2004$ & 29 & $4 / 16 / 2008$ & 33.17 & -4.2 \\
\hline 08N04W06CCB1 & 352028 & 912847 & $3 / 18 / 2004$ & 16.16 & $4 / 16 / 2008$ & 12.81 & 3.4 \\
\hline 08N05W32CBC1 & 351616 & 913417 & $3 / 18 / 2004$ & 1.5 & $4 / 16 / 2008$ & 3.46 & -2.0 \\
\hline \multicolumn{8}{|c|}{ Woodruff County } \\
\hline 04N03W03AB1 & 350021 & 911820 & $4 / 01 / 2004$ & 13.48 & $4 / 11 / 2008$ & 9.63 & 3.9 \\
\hline 05N01W13CDC1 & 350244 & 910331 & $4 / 13 / 2004$ & 74.1 & $4 / 17 / 2008$ & 76.6 & -2.5 \\
\hline 05N01W31CCC1 & 350106 & 910900 & $4 / 13 / 2004$ & 59.1 & $4 / 17 / 2008$ & 61.2 & -2.1 \\
\hline 05N02W20DCB1 & 350208 & 911356 & $4 / 01 / 2004$ & 13.94 & $4 / 11 / 2008$ & 11.87 & 2.1 \\
\hline 05N03W25DDB1 & 350133 & 911531 & $4 / 13 / 2004$ & 12.7 & $4 / 17 / 2008$ & 10.6 & 2.1 \\
\hline 05N04W12DBA1 & 350427 & 912211 & $4 / 01 / 2004$ & 4.67 & $4 / 15 / 2008$ & 2.94 & 1.7 \\
\hline 06N01W06BAB1 & 351048 & 910835 & $4 / 01 / 2004$ & 32.47 & $4 / 11 / 2008$ & 36.73 & -4.3 \\
\hline 06N02W19AAA1 & 350802 & 911419 & $4 / 14 / 2004$ & 44.7 & $4 / 17 / 2008$ & 45.9 & -1.2 \\
\hline 06N03W31BCB1 & 350623 & 912144 & $4 / 01 / 2004$ & 2.26 & $4 / 15 / 2008$ & 1.5 & 0.8 \\
\hline 06N04W22BDA1 & 350807 & 912428 & $4 / 13 / 2004$ & 4.9 & $4 / 17 / 2008$ & -1 & 5.9 \\
\hline 07N01W04ACB1 & 351541 & 910626 & $4 / 13 / 2004$ & 60.3 & $4 / 17 / 2008$ & 64.2 & -3.9 \\
\hline
\end{tabular}


Appendix 2. Information pertaining to water levels measured in 2004 and 2008 in the Mississippi River Valley alluvial aquifer in eastern Arkansas.-Continued

[USGS, U.S. Geological Survey; NRCS, Natural Resources Conservation Service, ANRC, Arkansas Natural Resources Commission; Horizontal coordinate information is referenced to the North American Datum of 1983 (NAD83); *, control wells for duplicate measurements by USGS and NRCS for quality assurance]

\begin{tabular}{|c|c|c|c|c|c|c|c|}
\hline Station name & $\begin{array}{c}\text { Latitude } \\
\text { (degrees, } \\
\text { minutes, } \\
\text { seconds) }\end{array}$ & $\begin{array}{c}\text { Longitude } \\
\text { (degrees, } \\
\text { minutes, } \\
\text { seconds) }\end{array}$ & $\begin{array}{l}2004 \text { water- } \\
\text { level date }\end{array}$ & $\begin{array}{c}2004 \text { depth } \\
\text { to water } \\
\text { (feet below } \\
\text { land-surface } \\
\text { datum) }\end{array}$ & $\begin{array}{l}2008 \text { water- } \\
\text { level date }\end{array}$ & $\begin{array}{c}2008 \text { depth } \\
\text { to water } \\
\text { (feet below } \\
\text { land-surface } \\
\text { datum) }\end{array}$ & $\begin{array}{c}\text { Water-level } \\
\text { difference } \\
2004 \text { to } 2008 \\
\text { (feet) }\end{array}$ \\
\hline \multicolumn{8}{|c|}{ Woodruff County-Continued } \\
\hline 07N03W06BAC1 & 351607 & 912109 & $3 / 24 / 2004$ & 21 & $3 / 21 / 2008$ & 23.5 & -2.5 \\
\hline 07N03W19AAA1 & 351335 & 912025 & $4 / 01 / 2004$ & 11.7 & $4 / 15 / 2008$ & 8.39 & 3.3 \\
\hline 07N03W31BBA1 & 351152 & 912103 & $4 / 13 / 2004$ & 14.7 & $4 / 17 / 2008$ & 7.7 & 7.0 \\
\hline 08N01W06DDD1 & 352028 & 910747 & $4 / 01 / 2004$ & 42.23 & $4 / 11 / 2008$ & 46.14 & -3.9 \\
\hline 08N02W27DDB1 & 351711 & 911107 & $4 / 13 / 2004$ & 25.5 & $4 / 17 / 2008$ & 27.5 & -2.0 \\
\hline 08N02W31DDD1 & 351611 & 911411 & $4 / 01 / 2004$ & 5.36 & $4 / 11 / 2008$ & 1.66 & 3.7 \\
\hline 08N03W31AAD1 & 351655 & 912028 & $4 / 01 / 2004$ & 21.77 & $4 / 15 / 2008$ & 22.78 & -1.0 \\
\hline 09N03W28ABB1 & 352310 & 911845 & $4 / 13 / 2004$ & 16.9 & $4 / 17 / 2008$ & 17.6 & -0.7 \\
\hline 09N03W29AAD1 & 352258 & 911921 & $4 / 01 / 2004$ & 20.6 & $4 / 15 / 2008$ & 18.9 & 1.7 \\
\hline 09N03W32ACA1 & 352205 & 911936 & $4 / 14 / 2004$ & 19.3 & $4 / 17 / 2008$ & 16.9 & 2.4 \\
\hline
\end{tabular}


Appendix 3. Specific conductance and temperature data from wells completed in the Mississippi River Valley alluvial aquifer in eastern Arkansas, summer 2008.

[Horizontal coordinate information is referenced to the North American Datum of 1983 (NAD83); $\mu \mathrm{S} / \mathrm{cm}$, microsiemens per centimeter at 25 degrees Celsius]

\begin{tabular}{|c|c|c|c|c|c|}
\hline Station name & $\begin{array}{c}\text { Latitude } \\
\text { (degrees, } \\
\text { minutes, } \\
\text { seconds) }\end{array}$ & $\begin{array}{c}\text { Longitude } \\
\text { (degrees, } \\
\text { minutes, } \\
\text { seconds) }\end{array}$ & Date & $\begin{array}{c}\text { Specific } \\
\text { conductance } \\
(\mu \mathrm{S} / \mathrm{cm})\end{array}$ & $\begin{array}{c}\text { Temperature } \\
\text { (degrees Celsius) }\end{array}$ \\
\hline \multicolumn{6}{|c|}{ Arkansas County } \\
\hline 02S04W15ADA1 & 343150 & 912452 & $6 / 23 / 2008$ & 884 & 19.1 \\
\hline 02S04W15BB1 & 343201 & 912553 & $6 / 23 / 2008$ & 809 & 19.1 \\
\hline 04S05W03BDB1 & 342309 & 913215 & $6 / 23 / 2008$ & 1,140 & 19.7 \\
\hline 07S03W07BBC1 & 340607 & 912322 & $6 / 23 / 2008$ & 845 & 19.1 \\
\hline \multicolumn{6}{|c|}{ Ashley County } \\
\hline 16S04W02DD1 & 332001 & 912845 & $6 / 19 / 2008$ & 546 & 19.7 \\
\hline 16S06W27BAB1 & 331729 & 914240 & $6 / 19 / 2008$ & 849 & 19.5 \\
\hline 16S06W35BD1 & 331622 & 914141 & $6 / 19 / 2008$ & 733 & 22.7 \\
\hline 18S08W12BDA1 & 330944 & 915253 & $6 / 19 / 2008$ & 832 & 20.4 \\
\hline \multicolumn{6}{|c|}{ Chicot County } \\
\hline 14S03W32DCB1 & 332613 & 912551 & $6 / 19 / 2008$ & 393 & 23.7 \\
\hline 15S03W06BCD1 & 332536 & 912712 & $6 / 19 / 2008$ & 405 & 19.3 \\
\hline 17S01W11AA1 & 331408 & 910953 & $6 / 19 / 2008$ & 639 & 22.1 \\
\hline 17S02W01CBA1 & 331456 & 911550 & $6 / 19 / 2008$ & 883 & 19.9 \\
\hline 17S02W02DA1 & 331457 & 911612 & $6 / 19 / 2008$ & 879 & 21.4 \\
\hline 17S02W11BC1 & 331415 & 911708 & $6 / 19 / 2008$ & 1,000 & 20.6 \\
\hline 18S02W22DCB1 & 330647 & 911747 & $6 / 19 / 2008$ & 1,020 & 20.1 \\
\hline \multicolumn{6}{|c|}{ Clay County } \\
\hline 19N08E29BCB1 & 361520 & 901452 & $6 / 26 / 2008$ & 347 & 17.9 \\
\hline 21N04E34DDC1 & 362445 & 903729 & $6 / 26 / 2008$ & 466 & 18.3 \\
\hline \multicolumn{6}{|c|}{ Craighead County } \\
\hline 13N02E35DC1 & 354221 & 905053 & $6 / 25 / 2008$ & 783 & 18.2 \\
\hline 16N07E32AB1 & 355826 & 902155 & $6 / 25 / 2008$ & 325 & 17.5 \\
\hline \multicolumn{6}{|c|}{ Crittenden County } \\
\hline 06N07E12DAA1 & 350900 & 901735 & $6 / 25 / 2008$ & 469 & 17.7 \\
\hline 07N07E22AA1 & 351320 & 902039 & $6 / 25 / 2008$ & 454 & 17.4 \\
\hline \multicolumn{6}{|c|}{ Cross County } \\
\hline 07N01E10BB1 & 351450 & 905910 & $6 / 24 / 2008$ & 1,010 & 18.1 \\
\hline 07N03E06BAC1 & 351516 & 904915 & $6 / 24 / 2008$ & 819 & 17.9 \\
\hline 09N05E08AA1 & 352537 & 903435 & $6 / 25 / 2008$ & 552 & 17.9 \\
\hline 09N05E32BDB1 & 352151 & 903512 & $6 / 25 / 2008$ & 540 & 17.1 \\
\hline \multicolumn{6}{|c|}{ Desha County } \\
\hline 10S03W22DB1 & 334913 & 911638 & $6 / 20 / 2008$ & 1,680 & 19.3 \\
\hline 10S04W16DDD1 & 334923 & 913012 & $6 / 20 / 2008$ & 877 & 19.0 \\
\hline 13S02W27DB1 & 333216 & 911722 & $6 / 20 / 2008$ & 2,020 & 19.1 \\
\hline 13S03W10DA1 & 333505 & 912306 & $6 / 20 / 2008$ & 725 & 19.4 \\
\hline
\end{tabular}


Appendix 3. Specific conductance and temperature data from wells completed in the Mississippi River Valley alluvial aquifer in eastern Arkansas, summer 2008._-Continued

[Horizontal coordinate information is referenced to the North American Datum of 1983 (NAD83); $\mu$ S/cm, microsiemens per centimeter at 25 degrees Celsius]

\begin{tabular}{|c|c|c|c|c|c|}
\hline Station name & $\begin{array}{c}\text { Latitude } \\
\text { (degrees, } \\
\text { minutes, } \\
\text { seconds) }\end{array}$ & $\begin{array}{c}\text { Longitude } \\
\text { (degrees, } \\
\text { minutes, } \\
\text { seconds) }\end{array}$ & Date & $\begin{array}{c}\text { Specific } \\
\text { conductance } \\
(\mu \mathrm{S} / \mathrm{cm})\end{array}$ & $\begin{array}{c}\text { Temperature } \\
\text { (degrees Celsius) }\end{array}$ \\
\hline \multicolumn{6}{|c|}{ Drew County } \\
\hline 11S04W07BDA1 & 334555 & 913256 & $6 / 18 / 2008$ & 269 & 19.1 \\
\hline 13S04W31AD1 & 333150 & 913248 & $6 / 18 / 2008$ & 313 & 19.5 \\
\hline 13S04W32DAA1 & 333134 & 913127 & $6 / 18 / 2008$ & 331 & 18.6 \\
\hline \multicolumn{6}{|c|}{ Greene County } \\
\hline 17N04E30CDC1 & 360409 & 904218 & $6 / 26 / 2008$ & 1,020 & 17.6 \\
\hline \multicolumn{6}{|c|}{ Jackson County } \\
\hline 09N01W20BDD1 & 352338 & 910805 & $6 / 26 / 2008$ & 503 & 18.4 \\
\hline 13N01W30DD1 & 354332 & 910835 & $6 / 26 / 2008$ & 445 & 17.7 \\
\hline \multicolumn{6}{|c|}{ Jefferson County } \\
\hline 03S09W18CC2 & 342656 & 920139 & $6 / 18 / 2008$ & 997 & 18.9 \\
\hline 04S09W29ACB1 & 342531 & 920000 & $6 / 18 / 2008$ & 610 & 18.6 \\
\hline 06S08W28BDB1 & 341030 & 915458 & $6 / 18 / 2008$ & 471 & 18.8 \\
\hline \multicolumn{6}{|c|}{ Lawrence County } \\
\hline 16N02E34BDA1 & 355839 & 905150 & $6 / 26 / 2008$ & 445 & 18.0 \\
\hline \multicolumn{6}{|c|}{ Lee County } \\
\hline 01N03E26BD1 & 344003 & 904545 & $6 / 23 / 2008$ & 668 & 18.1 \\
\hline 02N03E04BBC1 & 344859 & 904804 & $6 / 23 / 2008$ & 581 & 17.8 \\
\hline \multicolumn{6}{|c|}{ Lincoln County } \\
\hline 08S05W25AD1 & 340006 & 913218 & $6 / 20 / 2008$ & 1,210 & 18.7 \\
\hline 08S07W21CBB1 & 340042 & 914847 & $6 / 18 / 2008$ & 111 & 19.1 \\
\hline 09S05W01ADA1 & 335758 & 913259 & $6 / 18 / 2008$ & 388 & 19.3 \\
\hline 09S06W03CBA1 & 335739 & 914230 & $6 / 18 / 2008$ & 539 & 18.3 \\
\hline 09S06W04BCD1 & 335821 & 914346 & $6 / 18 / 2008$ & 393 & 19.5 \\
\hline \multicolumn{6}{|c|}{ Lonoke County } \\
\hline 02N07W02BBA1 & 344957 & 914338 & $6 / 17 / 2008$ & 876 & 21.3 \\
\hline \multicolumn{6}{|c|}{ Mississippi County } \\
\hline 12N08E07CC1 & 354025 & 901655 & $6 / 25 / 2008$ & 571 & 18.1 \\
\hline 14N10E22BB1 & 354941 & 900101 & $6 / 25 / 2008$ & 559 & 17.5 \\
\hline \multicolumn{6}{|c|}{ Monroe County } \\
\hline 01N02W24ABA1 & 344128 & 910951 & $6 / 24 / 2008$ & 777 & 18.7 \\
\hline 03N02W31ADC1 & 344958 & 911447 & $6 / 24 / 2008$ & 330 & 18.6 \\
\hline \multicolumn{6}{|c|}{ Phillips County } \\
\hline 04S01E01AD1 & 342238 & 905700 & $6 / 24 / 2008$ & 721 & 18.6 \\
\hline 04S01E23CCA1 & 341931 & 905853 & $6 / 24 / 2008$ & 741 & 18.4 \\
\hline \multicolumn{6}{|c|}{ Poinsett County } \\
\hline 10N02E13BCC1 & 352949 & 905026 & $6 / 25 / 2008$ & 941 & 17.8 \\
\hline
\end{tabular}


Appendix 3. Specific conductance and temperature data from wells completed in the Mississippi River Valley alluvial aquifer in eastern Arkansas, summer 2008. - Continued

[Horizontal coordinate information is referenced to the North American Datum of 1983 (NAD83); $\mu \mathrm{S} / \mathrm{cm}$, microsiemens per centimeter at 25 degrees Celsius]

\begin{tabular}{|c|c|c|c|c|c|}
\hline Station name & $\begin{array}{c}\text { Latitude } \\
\text { (degrees, } \\
\text { minutes, } \\
\text { seconds) }\end{array}$ & $\begin{array}{c}\text { Longitude } \\
\text { (degrees, } \\
\text { minutes, } \\
\text { seconds) }\end{array}$ & Date & $\begin{array}{c}\text { Specific } \\
\text { conductance } \\
(\mu \mathrm{S} / \mathrm{cm})\end{array}$ & $\begin{array}{c}\text { Temperature } \\
\text { (degrees Celsius) }\end{array}$ \\
\hline \multicolumn{6}{|c|}{ Poinsett County-Continued } \\
\hline 11N02E27CB1 & 353319 & 905223 & $6 / 25 / 2008$ & 642 & 18.0 \\
\hline 11N05E26CAD1 & 353253 & 903143 & $6 / 25 / 2008$ & 583 & 18.1 \\
\hline \multicolumn{6}{|c|}{ Prairie County } \\
\hline 01N06W30BBC1 & 344104 & 914154 & $6 / 17 / 2008$ & 666 & 21.9 \\
\hline \multicolumn{6}{|c|}{ St. Francis County } \\
\hline 04N01W28CDD1 & 345535 & 910634 & $6 / 24 / 2008$ & 752 & 18.1 \\
\hline 06N02E13DC1 & 350803 & 905009 & $6 / 24 / 2008$ & 664 & 18.0 \\
\hline \multicolumn{6}{|c|}{ Woodruff County } \\
\hline 07N02W04ADA1 & 351550 & 911201 & $6 / 26 / 2008$ & 553 & 17.4 \\
\hline
\end{tabular}

Publishing support provided by: Lafayette and Rolla Publishing Service Centers

For more information concerning the research described in the report

U.S. Geological Survey

Arkansas Water Science Center

401 Hardin Road

Little Rock, AR 72211-3528

(501) 228-3600

http://ar.water.usgs.gov 
